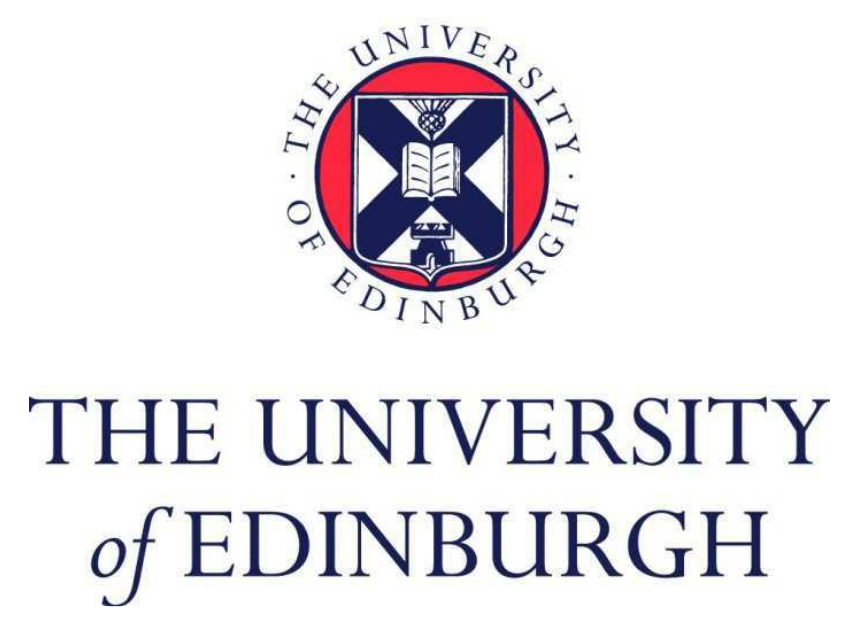

This thesis has been submitted in fulfilment of the requirements for a postgraduate degree (e.g. PhD, MPhil, DClinPsychol) at the University of Edinburgh. Please note the following terms and conditions of use:

This work is protected by copyright and other intellectual property rights, which are retained by the thesis author, unless otherwise stated.

A copy can be downloaded for personal non-commercial research or study, without prior permission or charge.

This thesis cannot be reproduced or quoted extensively from without first obtaining permission in writing from the author.

The content must not be changed in any way or sold commercially in any format or medium without the formal permission of the author.

When referring to this work, full bibliographic details including the author, title, awarding institution and date of the thesis must be given. 


\title{
The Effect of Pressure on Porous Materials
}

\author{
Charles J. McMonagle
}

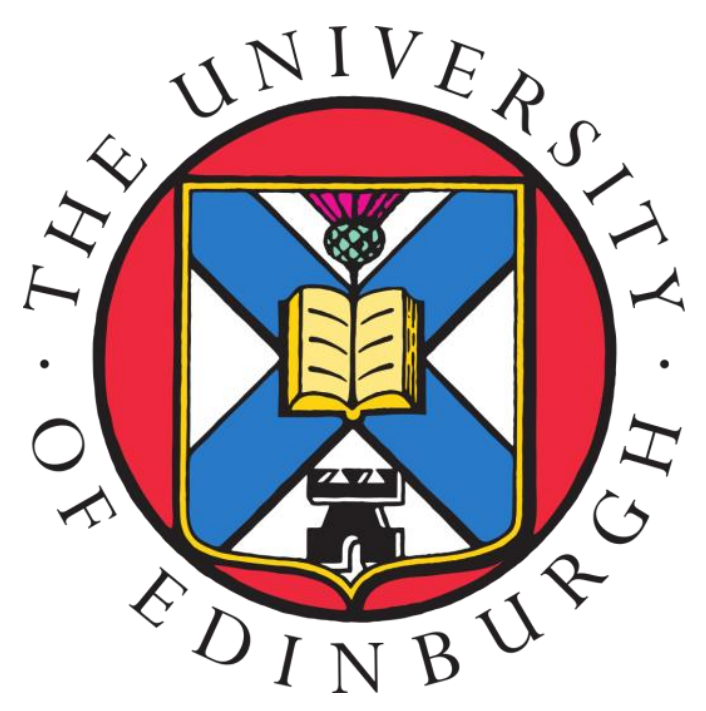

A thesis submitted in fulfilment of the requirements for the degree of Doctor of Philosophy to the School of Chemistry

The University of Edinburgh 


\section{Declaration}

I declare that this thesis was composed by myself and that the work contained herein is my own except where explicitly stated otherwise in the text. This work has not been submitted for any other degree or professional qualification.

Charles J. McMonagle 


\section{Abstract}

Research to design and synthesise new porous materials is a rapidly growing field with thousands of new systems proposed every year due to their potential use in a multitude of application in a wide range of fields. Pressure is a powerful tool for the characterisation of structure-property relationships in these materials, the understanding of which is key to unlocking their full potential. In this thesis we investigate a range of porous materials at a range of pressures.

Over time the chemical architecture and complexity of porous materials has increased. Although some systems display remarkable stability to high-pressures, which we generally think of as being above $1 \mathrm{GPa}(10,000 \mathrm{bar})$, in general, the compressibility of porous materials have increased substantially over the last 10 years, rendering most unstable at GPa pressures. Here we present new methods for investigating porous materials at much more moderate pressures (100's of bar), alongside more traditional high-pressure methods (diamond anvil cell techniques), finishing with gas sorption studies in a molecular based porous material.

Here, the design and development of a new moderate pressure sapphire capillary cell for the small molecule beamline I19 at the Diamond Light Source is described. This cell allowed access to pressures of more than 1000 bar regularly with a maximum operating pressure of 1500 bar with very precise pressure control $(<10$ bar) on both increasing and decreasing pressure. This cell closes the gap between ambient pressure and the lowest pressures attainable using a diamond anvil cell (DAC), which is generally above $0.2 \mathrm{GPa}$ (2000 bar). Along with the development of the sapphire capillary pressure cell, the compression to 1000 bar of the small organic sample molecule Hexamethylenetetramine (hexamine, $\mathrm{C}_{6} \mathrm{H}_{12} \mathrm{~N}_{4}$ ) and its deuterated form $\left(\mathrm{C}_{6} \mathrm{D}_{12} \mathrm{~N}_{4}\right)$ was determined, demonstrating the precision possible using this cell.

Solvent uptake into porous materials can induce large structural changes at 100's of bar. In the case of the Sc-based Metal-organic framework (MOF), $\mathrm{Sc}_{2} \mathrm{BDC}_{3}$ (BDC =1,4-benzenedicarboxylate), we used the sapphire capillary pressure cell to study changes in the framework structure on the uptake of $n$-pentane and isopentane. 
This work shows how the shape and smaller size of $n$-pentane facilitated the swelling of the framework that could be used to explain the increase in stability of the MOF to applied pressure.

The effect of pressure on the previously unreported Cu-framework bis[1-(4pyridyl)butane-1,3-dione]copper(II) (CuPyr-I) was investigated using highpressure single-crystal diffraction techniques (DAC). CuPyr-I was found to exhibit high-pressure and low-temperature phase transitions, a pressure induced JahnTeller switch (which was hydrostatic medium dependent), piezochromism, and negative linear compressibility. Although each of these phenomena has been reported numerous times in a range of materials, this is to the best of our knowledge the first example to have been observed within the same material.

The final two chapters investigate the exceptional thermal, chemical, and mechanical stability of a porous molecular crystal system (PMC) prepared by the co-crystallisation of a cobalt phthalocyanine derivative and a fullerene $\left(\mathrm{C}_{60}\right.$ or $\mathrm{C}_{70}$ ). The stabilising fullerene is captured in the cavity between two phthalocyanines in a ball and socket arrangement. These PMCs retain their porous structure: on the evacuation of solvent of crystalisation; on heating to over 500 $\mathrm{K}$; on prolonged immersion in boiling aqueous acid, base, and water; and at extreme pressures of up to $5.85 \mathrm{GPa}$, the first reported high-pressure study of a PMC. the reactive cobalt cation is accessible via the massive interconnected voids, $\left(8 \mathrm{~nm}^{3}\right)$, as demonstrated by the adsorption and binding of $\mathrm{CO}$ and $\mathrm{O}_{2}$ to the empty metal site using in situ crystallographic methods available at beamline I19, Diamond Light Source. 


\section{Lay Summary}

Porous materials have interconnected molecular sized voids or channels in their molecular structure. These materials are potentially useful candidates for industrial gas/liquid-separations and gas-storage. Many of these processes are conducted at high pressures and so understanding how these materials behave at pressure is important. Single-crystal X-ray diffraction is one of the most powerful tools for determining atomic structure and this thesis outlines new and existing methods for performing experiments over a very wide range of pressures using this technique. 


\section{Acknowledgements}

Firstly Stephen, thank you for providing such amazing support throughout the years both professionally and personally. Your steadfast backing of me and my approach has always given me the confidence to press on, even on the darker days. These three and quite a bit years have flown by, but I look forward to many years of friendship in the future wherever we both end up. Simon, it has been an honour to learn from you over not only this $\mathrm{PhD}$ but also during my degree. You are perhaps one of the main reasons I started down this road in the first place, and I will always remember your patient teaching and generous hospitality.

A massive thank you must also go to the members past and present of the Moggach and Parsons groups. To Chris for all of the practical advice on how to get stuff done and never a dull moment. To Scot, Jack, Andy, Claire and Jorge for such a great fun office and endless humour, it was a real pleasure to get to know you all. Thank you to the 'new starts' Helen, Jacob, Nico, Matt, and Jonny for keeping the Office 85 spirit alive and putting up with me while writing up. To Gary for all the lunch time chats, crystallographic advise, and endless positivity. And to everyone one at Kings who made the years fly by.

Being based in Edinburgh made the generosity of all the staff on I19 while I was there very special to me. To Dave, for quietly supervising and openly encouraging me to play around with my ideas. To Mark and Anna, for being one of the loveliest couples I know and both endlessly resourceful. Mark this project really would not have be possible without your help and I will always be grateful for your total enthusiasm.

To my parents, sister and the rest of the family thank you for your enduring love and support. And finally, Tiffany, your love, patience, and understanding have made this as much your achievement as mine. This is the end of a chapter for me but the next has already started, and I cannot imagine anyone I would rather embark on that adventure than you. 


\section{Publications}

Burt, L. A., Bezzu, C. G., McMonagle, C. J., Moggach, S. A. \& McKeown, N. B. A hindered subphthalocyanine that forms crystals with included aromatic solvent but will not play ball with $C_{60}$. J. Porphyrins Phthalocyanines 20, 1034-1040, doi:10.1142/s1088424616500528 (2016). 


\section{Pressure Conversion}

\begin{tabular}{|c|c|c|c|c|c|c|c|}
\hline bar & kbar & $\mathbf{P a}^{[\mathbf{a}]}$ & MPa & GPa & $\mathbf{a t m}^{[\mathbf{b}]}$ & $\operatorname{Torr}^{[\mathrm{c}]}$ & $\operatorname{psi}^{[\mathrm{d}]}$ \\
\hline 1 & 0.001 & $1 \times 10^{5}$ & 0.1 & 0.0001 & 0.987 & 750 & 14.504 \\
\hline 2 & 0.002 & $2 \times 10^{5}$ & 0.2 & 0.0002 & 1.974 & 1,500 & 29.008 \\
\hline 3 & 0.003 & $3 \times 10^{5}$ & 0.3 & 0.0003 & 2.961 & 2,250 & 43.511 \\
\hline 4 & 0.004 & $4 \times 10^{5}$ & 0.4 & 0.0004 & 3.948 & 3,000 & 58.015 \\
\hline 5 & 0.005 & $5 \times 10^{5}$ & 0.5 & 0.0005 & 4.935 & 3,750 & 72.519 \\
\hline 10 & 0.01 & $1 \times 10^{6}$ & 1.0 & 0.001 & 9.87 & 7,501 & 145.038 \\
\hline 20 & 0.02 & $2 \times 10^{6}$ & 2.0 & 0.002 & 19.74 & $1.50 \times 10^{4}$ & 290.075 \\
\hline 30 & 0.03 & $3 \times 10^{6}$ & 3.0 & 0.003 & 29.61 & $2.25 \times 10^{4}$ & 435.113 \\
\hline 40 & 0.04 & $4 \times 10^{6}$ & 4.0 & 0.004 & 39.48 & $3.00 \times 10^{4}$ & 580.151 \\
\hline 50 & 0.05 & $5 \times 10^{6}$ & 5.0 & 0.005 & 49.35 & $3.75 \times 10^{4}$ & 725.189 \\
\hline 100 & 0.1 & $1 \times 10^{7}$ & 10 & 0.01 & 98.69 & $7.50 \times 10^{4}$ & $1.45 \times 10^{3}$ \\
\hline 200 & 0.2 & $2 \times 10^{7}$ & 20 & 0.02 & 197.4 & $1.50 \times 10^{5}$ & $2.90 \times 10^{3}$ \\
\hline 300 & 0.3 & $3 \times 10^{7}$ & 30 & 0.03 & 296.1 & $2.25 \times 10^{5}$ & $4.35 \times 10^{3}$ \\
\hline 400 & 0.4 & $4 \times 10^{7}$ & 40 & 0.04 & 394.8 & $3.00 \times 10^{5}$ & $5.80 \times 10^{3}$ \\
\hline 500 & 0.5 & $5 \times 10^{7}$ & 50 & 0.05 & 493.5 & $3.75 \times 10^{5}$ & $7.25 \times 10^{3}$ \\
\hline 600 & 0.6 & $6 \times 10^{7}$ & 60 & 0.06 & 592.2 & $4.50 \times 10^{5}$ & $8.70 \times 10^{3}$ \\
\hline 700 & 0.7 & $7 \times 10^{7}$ & 70 & 0.07 & 690.8 & $5.25 \times 10^{5}$ & $1.02 \times 10^{4}$ \\
\hline 800 & 0.8 & $8 \times 10^{7}$ & 80 & 0.08 & 789.5 & $6.00 \times 10^{5}$ & $1.16 \times 10^{4}$ \\
\hline 900 & 0.9 & $9 \times 10^{7}$ & 90 & 0.09 & 888.2 & $6.75 \times 10^{5}$ & $1.31 \times 10^{4}$ \\
\hline 1,000 & 1 & $1 \times 10^{8}$ & 100 & 0.1 & $0.98 \times 10^{3}$ & $7.50 \times 10^{5}$ & $1.45 \times 10^{4}$ \\
\hline 2,000 & 2 & $2 \times 10^{8}$ & 200 & 0.2 & $1.97 \times 10^{3}$ & $1.50 \times 10^{6}$ & $2.90 \times 10^{4}$ \\
\hline 3,000 & 3 & $3 \times 10^{8}$ & 300 & 0.3 & $2.96 \times 10^{3}$ & $2.25 \times 10^{6}$ & $4.35 \times 10^{4}$ \\
\hline 4,000 & 4 & $4 \times 10^{8}$ & 400 & 0.4 & $3.95 \times 10^{3}$ & $3.00 \times 10^{6}$ & $5.80 \times 10^{4}$ \\
\hline 5,000 & 5 & $5 \times 10^{8}$ & 500 & 0.5 & $4.93 \times 10^{3}$ & $3.75 \times 10^{6}$ & $7.25 \times 10^{4}$ \\
\hline 10,000 & 10 & $1 \times 10^{9}$ & 1,000 & 1 & $9.87 \times 10^{3}$ & $7.50 \times 10^{6}$ & $1.45 \times 10^{5}$ \\
\hline 20,000 & 20 & $2 \times 10^{9}$ & 2,000 & 2 & $1.97 \times 10^{4}$ & $1.50 \times 10^{7}$ & $2.90 \times 10^{5}$ \\
\hline 30,000 & 30 & $3 \times 10^{9}$ & 3,000 & 3 & $2.96 \times 10^{4}$ & $2.25 \times 10^{7}$ & $4.35 \times 10^{5}$ \\
\hline 40,000 & 40 & $4 \times 10^{9}$ & 4,000 & 4 & $3.95 \times 10^{4}$ & $3.00 \times 10^{7}$ & $5.80 \times 10^{5}$ \\
\hline 50,000 & 50 & $5 \times 10^{9}$ & 5,000 & 5 & $4.93 \times 10^{4}$ & $3.75 \times 10^{7}$ & $7.25 \times 10^{5}$ \\
\hline 100,000 & 100 & $1 \times 10^{10}$ & 10,000 & 10 & $9.87 \times 10^{4}$ & $7.50 \times 10^{7}$ & $1.45 \times 10^{6}$ \\
\hline
\end{tabular}

[a] Pa - Pascal the SI unit of pressure

[b] atm - The standard atmosphere

[c] Torr $-1 / 760 \mathrm{~atm} . \approx 1 \mathrm{mmHg}$

${ }^{\text {[d] }}$ Psi - Pound-force per square inch 


\section{List of Abbreviations}

1-MCP 1-methylcyclopropene

ADP Atomic Displacement Parameter

BDC benzene-1,4-dicarboxylate

BET Brunauer-Emmett-Teller theory

bipy $\quad 4,4^{\prime}$-bipyridyl

BTC benzene-1,3,5-tricarboxylate

CCD Charge-Coupled Device

COF Covalent Organic Framework

CSD Cambridge Structural Database

DAC Diamond Anvil Cell

EH Experimental Hutch

EoS Equation of State

EPICS Experimental Physics and Industrial Control System

FC-70 Fluorinert, a bulky perfluorinated amine

FC-77 Fluorinert, a bulky perfluorinated amine

FSO Full-Scale Output

GCMC Grand Canonical Monte Carlo methods

HKUST Hong Kong University of Science and Technology

ID Internal Diameter

IPA Iso-Propyl Alcohol

JT Jahn-Teller

K Bulk Modulus

MeIm 2-methylimidazolate

MEW 16:3:1 methanol:ethanol:water mixture

MOF Metal Organic Framework

NLC Negative Linear Compressibility

NMC Nanoporous Molecular Crystal

OD Outside Diameter

PDIC 1,4-phenylenediisocyanide

PMC Porous Molecular Crystal

PNC Phthalocyanine Nanoporous Crystal

PSM Post Synthetic Modification

PTM Pressure Transmitting Medium

PUNC Phthalocyanine Unsolvated Nanoporous Crystal

pXRD Powder X-Ray Diffraction

Py Pyridine

SBU Secondary Building Unit

scXRD Single-Crystal X-Ray Diffraction

STAM St.Andrews MOF

TGA Thermogravimetric Analysis

ZIF Zeolitic Imidazolate Framework 


\section{Table of contents}

- Chapter 1 - Introduction ........................................................ 1

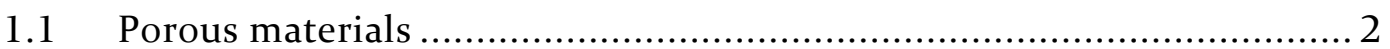

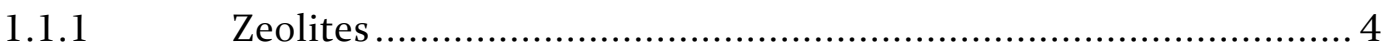

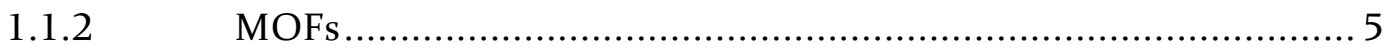

1.1.3 Porous Molecular Crystal (PMC) …......................................... 8

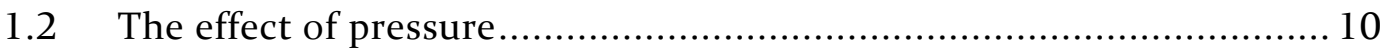

1.3 Examples of the effects of pressure ................................................ 13

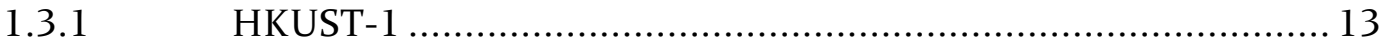

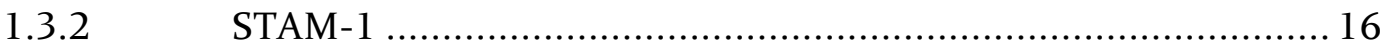

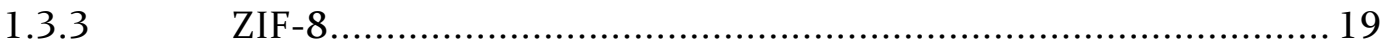

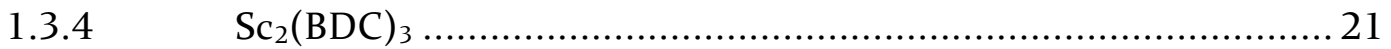

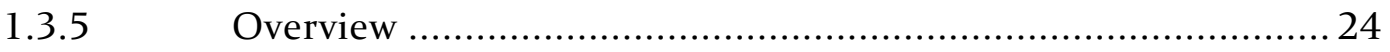

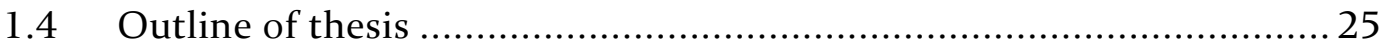

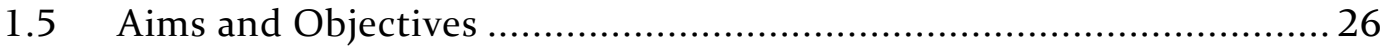

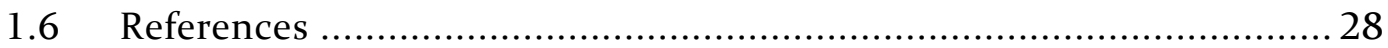

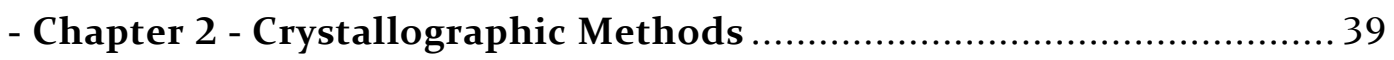

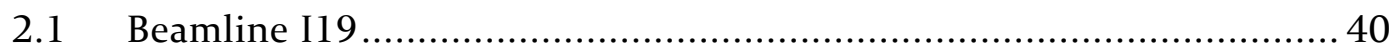

2.2 High-pressure crystallographic methods................................. 42

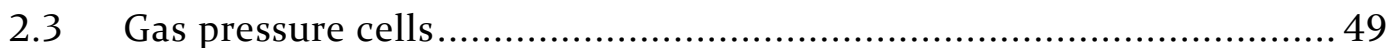

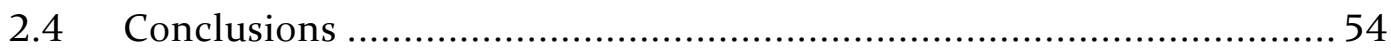

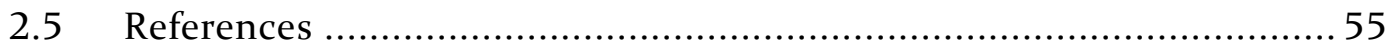

- Chapter 3 - Sapphire Capillary Pressure Cell for In-Situ Single Crystal Diffraction Measurements on Beamline I19 at Diamond Light Source.................................................. 61

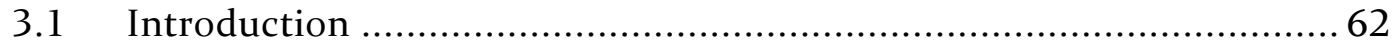

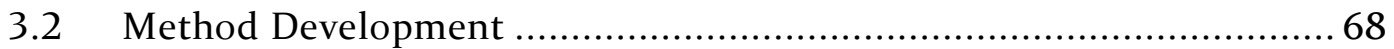

3.2.1 Sapphire capillary pressure cell design ................................69 69

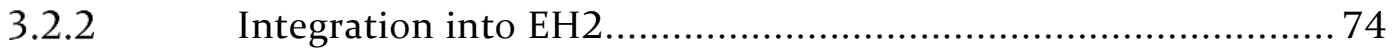

3.2.3 Collecting single-crystal X-ray diffraction data ......................76 


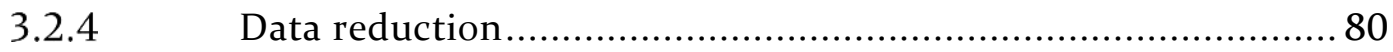

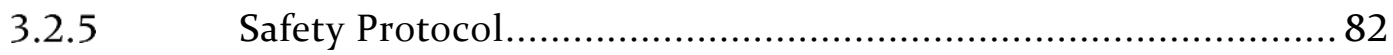

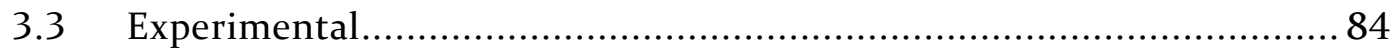

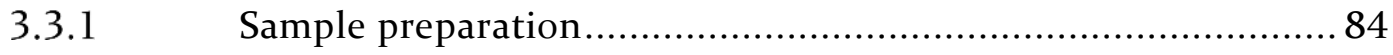

3.3.2 Laboratory single-crystal measurements............................. 84

3.3.3 Synchrotron single-crystal moderate pressure measurements... 84

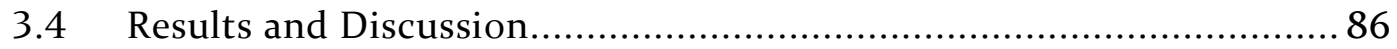

3.4.1 Ambient pressure structures of HMTH and HMTD ................. 86

3.4.2 The effect of pressure on HMTH and HMTD ......................... 88

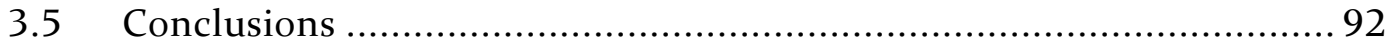

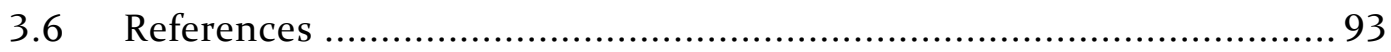

- Chapter 4 - The Effect of Pressure on $\mathrm{Sc}_{2} \mathrm{BDC}_{3}$ using the Sapphire Capillary Pressure Cell ................................................ 98

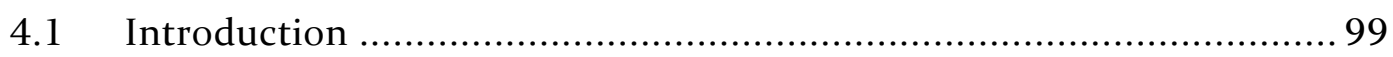

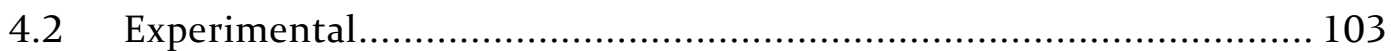

4.2.1 Synthesis of $\mathrm{Sc}_{2} \mathrm{BDC}_{3}$ via a solvothermal route..................... 103

4.2.2 Moderate pressure crystallographic studies ......................... 103

4.3 Results and Discussion.............................................................. 106

4.3.1 Unit cell compression .................................................... 106

4.3.2 The effect on $\mathrm{Sc}_{2} \mathrm{BDC}_{3}$ on uptake of isopentane .................... 107

4.3.3 The effect on $\mathrm{Sc}_{2} \mathrm{BDC}_{3}$ on uptake of $n$-pentane .................... 109

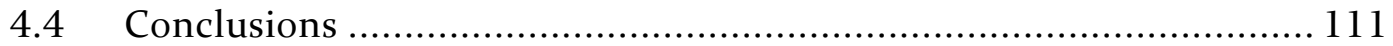

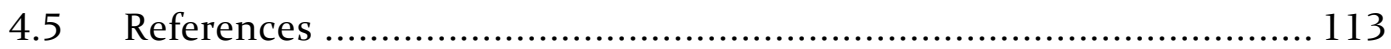

- Chapter 5 - The Effect of High Pressure on a Cu-Based Framework.. 115

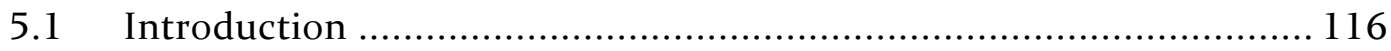

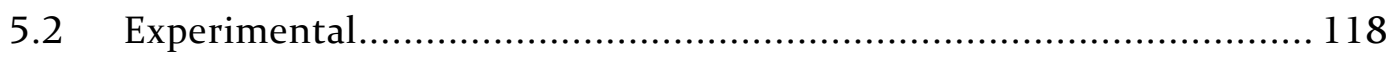

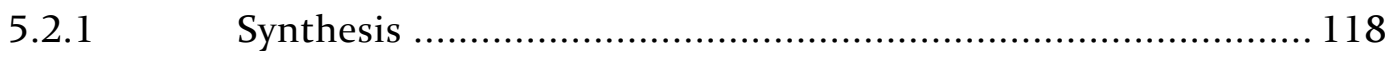

5.2.2 Single-Crystal X-ray Diffraction Experiments ...................... 118

5.2.3 Powder Diffraction Measurements....................................... 120

5.2.4 Thermogravimetric analysis (TGA) ................................ 120

5.3 Results and Discussion...................................................... 121 
5.3.1 High Pressure Experiments ............................................. 122

5.3.2 Void content as a function of pressure............................... 131

5.3.3 The effect of decreasing temperature on CuPyr-I.................. 134

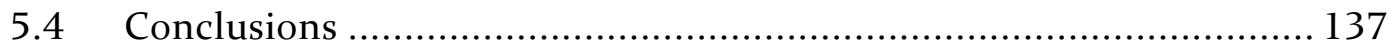

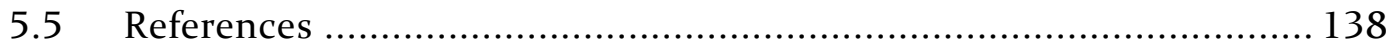

- Chapter 6 - The Effect of Pressure, Temperature, and Chemical Stability of Phthalocyanine Porous Molecular Crystals

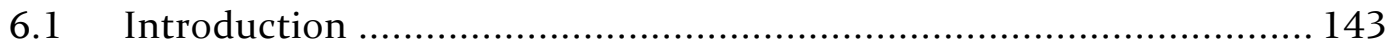

6.1.1 Porous Molecular Crystals ........................................... 143

6.1.2 Phthalocyanine Porous Molecular Crystals .......................... 145

6.1.3 A Note on Nomenclature ............................................ 149

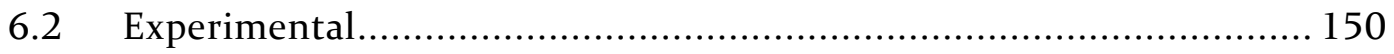

6.2.1 Equipment .......................................................... 150

6ynthesis ............................................................... 150

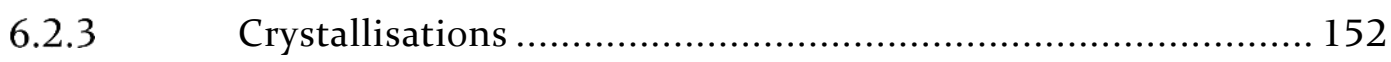

6.2.4 Permanent porosity determination .................................. 152

6.2.5 Thermal gravimetric analyses (TGA) .................................. 152

6.2.6 Powder X-ray diffraction................................................ 153

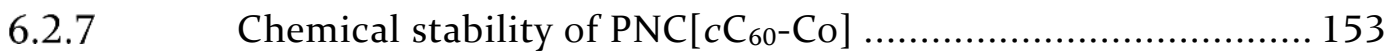

6.2.8 Laboratory single crystal measurements ............................ 153

6.2.9 High-pressure crystallographic studies .............................. 153

6.2.10 High pressure structure refinements ............................... 154

6.3 Results and Discussion...................................................... 156

6.3.1 Permanent Porosity ........................................................ 157

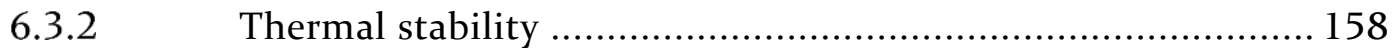

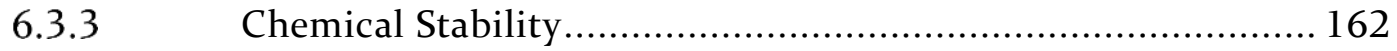

6.3.4 High Pressure Experiments .............................................. 164

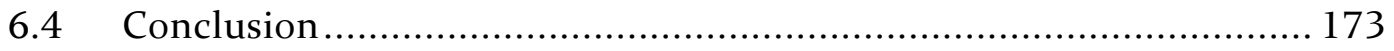

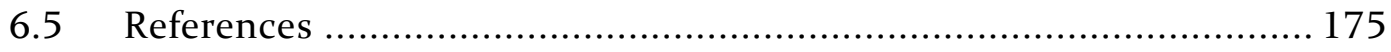


- Chapter 7 - Gas Absorption into Fullerene Stabilised

Phthalocyanine Porous Molecular Crystals 185

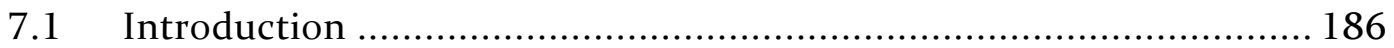

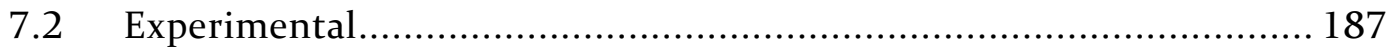

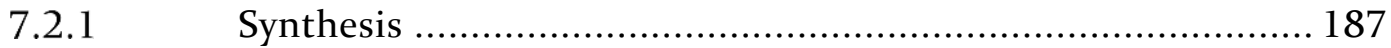

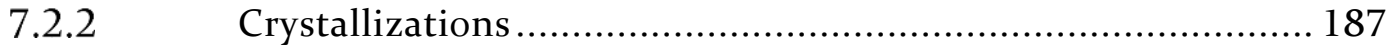

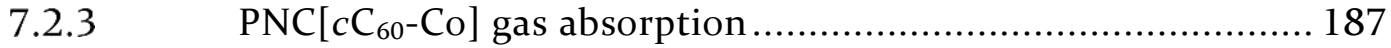

7.2.4 Cambridge structural database (CSD) searches ..................... 188

7.3 Results and Discussion............................................................. 189

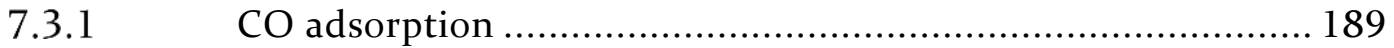

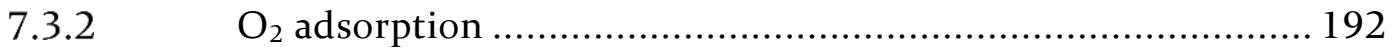

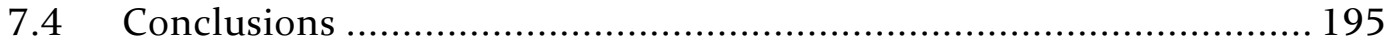

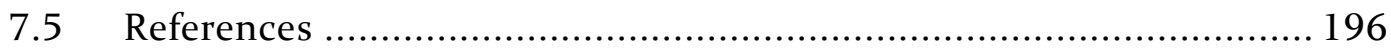

- Chapter 8 - Concluding Remarks and Further Work ......................... 198

8.1 Concluding Remarks and Further Work................................... 199 
- Chapter 1 -

\section{Introduction}




\subsection{Porous materials}

The International Union of Pure and Applied Chemistry (IUPAC) defines a porous solid as 'a solid with pores, i.e. cavities, channels or interstices, which are deeper than they are wide'. ${ }^{1}$ This broad definition results in the majority of materials being classed as porous making truly non-porous materials rare. Therefore it is much more useful to describe the nature and extent of the pores present rather than merely their existence.

The schematic in Figure 1.1 was used by IUPAC to classify different pore types that might be present in a material with regard to their availability to an external fluid or gas. Pores that are totally isolated, without connections to the outside surface of the body of the material are described as closed pores, (a). Closed pores affect many macroscopic properties of the material such as density, compressibility, and mechanical strength, however, they are not accessible and therefore cannot take part in gas adsorption. Open pores have continuous access to the external surface. These can be blind pores (open only at one end (b) \& (c)), or through pores (d), and can be of varying size, shape, and chemical nature. Close to, but different from porosity is the roughness of the external surface (e). By convention, a rough surface is not porous unless it has irregularities that are deeper than they are wide.

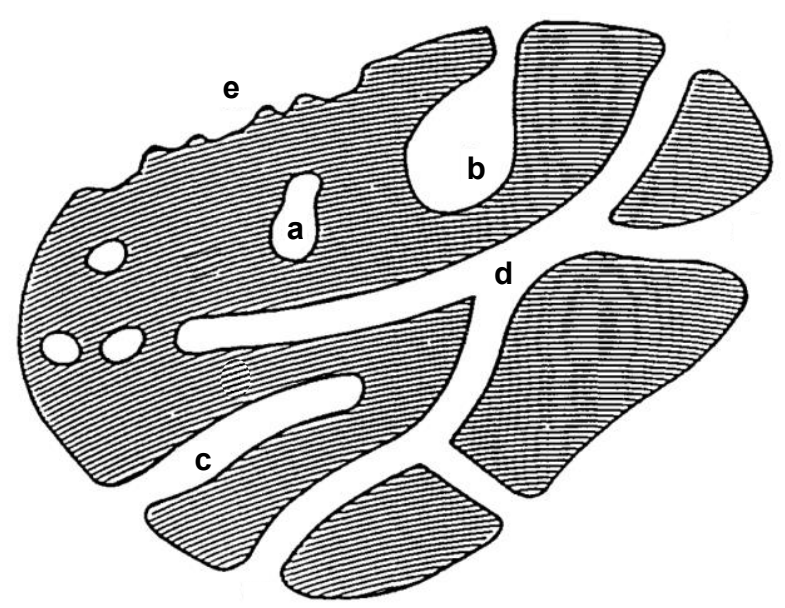

Figure 1.1 - Schematic cross-section of a porous solid. (a) Closed pore, (b) \& (c) Open blind pores (open only at one end), (d) through pores, and (e) surface roughness. Adapted from Rouquerol et al. 1994. 
In order to be of maximum use, any description of porosity should be as comprehensive as possible. Most importantly, the size and shape of the pores should be described. Pore size has a precise meaning when the geometrical shape of the pores is well defined and known (e.g. cylindrical, slit-shaped etc.). For most phenomena in which porous solids are involved the limiting size of a pore is the most critical. This is the smallest dimension of the pore, i.e. the width of a slitshaped pore or the diameter of a cylindrical pore. A size-dependent classification has been outlined by IUPAC with the following nomenclature:

i. micropores have widths smaller than $2 \mathrm{~nm}$;

ii. mesopores have widths between 2 and $50 \mathrm{~nm}$;

iii. macropores have widths larger than $50 \mathrm{~nm}$.

This classing system is not without its detractors and alternatives have been proposed. ${ }^{2}$ In the work presented in this thesis, we will focus on crystalline porous materials that contain open through pores that are an inherent feature of their structures. Materials of this nature include zeolites, zeotypes, porous carbons, metal-organic frameworks (MOFs), covalent organic frameworks (COFs), porous molecular crystals (PMCs), and many other smaller subclasses.

In recent years crystalline porous materials containing organic ligands or components have garnered considerable attention, in particular, MOFs and COFs. Much of this attention is due to potential applications in, but not limited to, heterogeneous catalysis, ${ }^{3-5}$ gas storage and release, ${ }^{6-12}$ chemical separations, ${ }^{13-15}$ drug delivery, ${ }^{16-18}$ and sensing. ${ }^{19-23}$ The pore structure of these materials is a key reason for their versatility as it can have a large effect on the resulting physical properties including density, thermal and electrical conductivity, ${ }^{24-26}$ magnetism, ${ }^{27}$ and luminescence. ${ }^{28}$ It is the link between pore structure and the mechanical, electronic, and chemical properties of porous materials that have led to their investigation as a function of pressure. 


\subsubsection{Zeolites}

Zeolites are porous crystalline materials constructed from silicon, aluminium, and oxygen that form a framework material with cavities and channels where cations, water, and/or small molecules may reside. Many of them occur naturally as minerals and are extensively mined in many parts of the world. The phenomenal success of zeolites in a variety of applications has led to an estimated global production in the range of 2.7 to 3.2 million metric tons a year. ${ }^{29}$ They are used in petrochemical cracking, ion-exchange (water softening and purification), and in the separation and extraction of gases and solvents.

Zeolites are crystalline aluminosilicates with open 3D framework structures built of linked $\mathrm{SiO}_{4}$ and $\mathrm{AlO}_{4}$ tetrahedra to form regular intra-crystalline cavities and channels of molecular dimensions. A defining feature of zeolites is that their frameworks are made up of 4-coordinated atoms forming tetrahedra. These tetrahedra are linked together by their corners to make a wide range of different framework types.

When aluminium, $\mathrm{Al}^{3+}$, replaces silicon, $\mathrm{Si}^{4+}$, in the framework there is an overall negative charge on the framework. Additional cations $\left(\mathrm{M}^{+}\right)$must therefore be

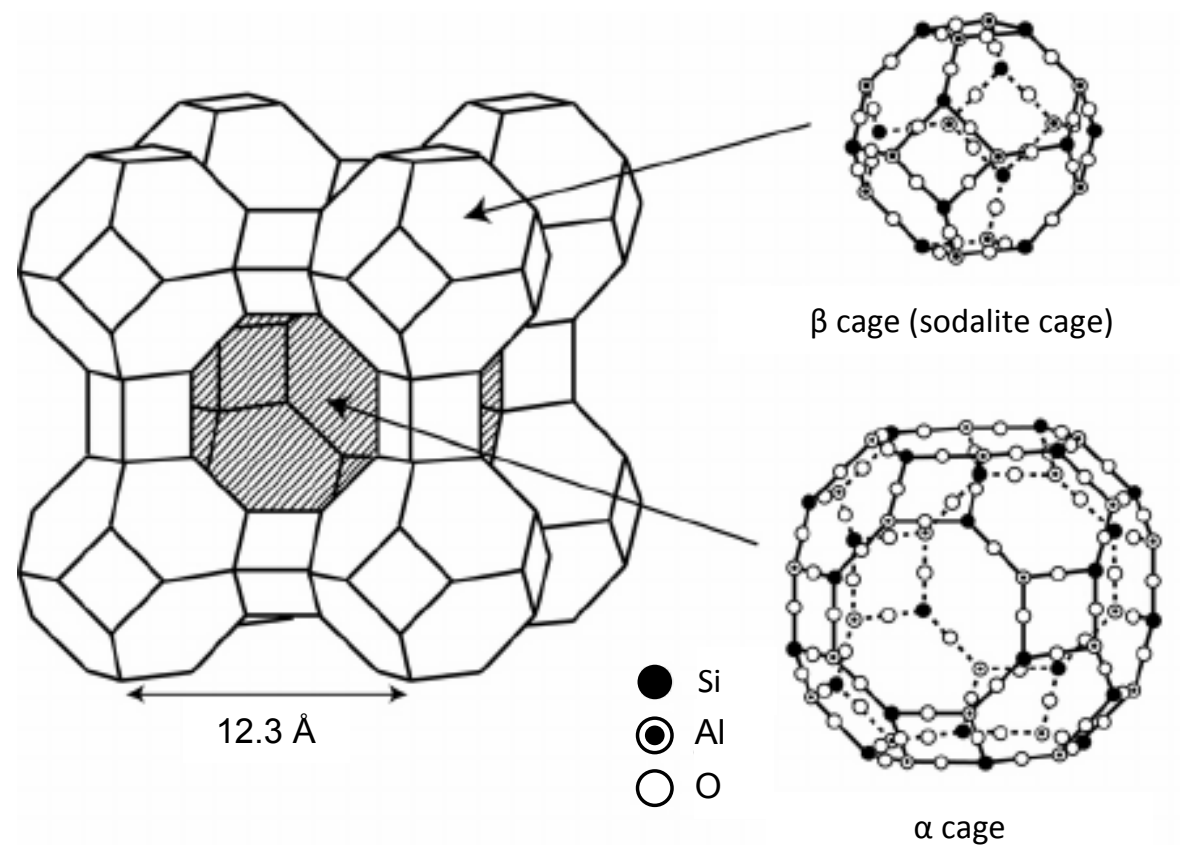

Figure 1.2 - Structure of zeolite Na-A with inserts of the $\alpha$ and $\beta$ cages. The cation and water are not shown for clarity. Adapted from Nakano \& Nozue, 2007. 
present to maintain an overall charge balance, with zeolites having the general chemical formula $\left(\mathrm{M}^{\mathrm{n}+}\right)_{\mathrm{x} / \mathrm{n}}\left(\mathrm{AlO}_{2}\right)_{\mathrm{x}}\left(\mathrm{SiO}_{2}\right)_{\mathrm{y}} \cdot \mathrm{Z}\left(\mathrm{H}_{2} \mathrm{O}\right)$. ${ }^{30}$ The cations and water molecules are only loosely bound to the framework and mobile. As a consequence, they can be exchanged readily. The Si/Al ratio can be varied in the majority of zeolites, either during the synthesis, or by post-synthetic dealumination treatment. Zeolites are widely accepted to obey Löwenstein's rule of "aluminium avoidance" forbidding formation of $-\mathrm{Al}-\mathrm{O}-\mathrm{Al}-$ linkages restricting the minimum Si/Al ratio of any zeolite to 1:1. ${ }^{31}$ Recently this has be challenged but a nonLöwensteinian zeolite is still to be synthetically realised. ${ }^{32}$

Zeolite A is an example of a typical zeolite, Figure 1.2. ${ }^{33}$ When the cation present is sodium, the composition of zeolite $A$ (referred to as $\mathrm{Na}-\mathrm{A}$ ) is $\mathrm{Na}_{12}\left[\mathrm{Al}_{12}\right.$ $\left.\mathrm{Si}_{12} \mathrm{O}_{48}\right] . \sim 27 \mathrm{H}_{2} \mathrm{O}$ under ambient pressure and temperature conditions. All forms of zeolite $\mathrm{A}$, regardless of the $\mathrm{Si} / \mathrm{Al}$ ratio, cations present or water content, have the Linde Type A (LTA) topology. The three-letter nomenclature for zeolites is based on the categorization scheme of the International Zeolite Association, whereby each unique topology is given a code. At present, there are 235 unique zeolite frameworks identified, and over 40 naturally occurring zeolite frameworks are known. ${ }^{34}$

\subsubsection{MOFs}

The purely inorganic nature of zeolites limits their potential chemical and structural diversity. By preparing materials with both inorganic and organic components, chemists have obtained a far greater degree of flexibility in the preparation of porous crystals. The term 'metal-organic framework' was first introduced by Omar Yaghi's group in 1995, and since this time there has been an explosion of research interest in the field of MOFs. ${ }^{35}$ The chemical and structural diversity offered by these systems has led to an exciting and diverse area of research combining supramolecular chemistry with crystallography, computational chemistry, mechanical and chemical engineering, materials science and many more disciplines. With more than 1,000 research papers published every year in the field and the fastestgrowing subset of the Cambridge Structural Database (CSD), with more than 81,000 
MOF structures (May 2017) and doubling every 3.9 years, the sheer volume of work on MOFs becomes apparent. ${ }^{36-38}$

As the field of MOFs has expanded there has been a wide variety of terminologies and definitions as to what a MOF is. This led to IUPAC setting up a task group to recommend a unified terminology for MOFs and coordination polymers using new or existing IUPAC nomenclature. ${ }^{39}$

The task group identified coordination polymers as an umbrella term for polymeric materials whose repeat units are coordination entities and defined as a "coordination compound with repeating coordination entities extending in 1, 2, or 3 dimensions". A coordination entity is an ion or neutral molecule that is composed of a central atom, usually that of a metal, to which is attached a surrounding array of other atoms or groups of atoms, each of which is called a ligand.

The task group then go on to define a coordination network, a subgroup of coordination polymer, as "a coordination compound extending through repeating coordination entities, in 1 dimension, but with cross-links between two or more individual chains, loops, or spiro-links, or a coordination compound extending through repeating coordination entities in 2 or 3 dimensions". This adds the requirement for higher dimensionality seen in network compounds.

And finally to defining MOFs as a subgroup of coordination networks as "a coordination network with organic ligands containing potential voids". This add the requirement for an organic component to the structure but formally there are no requirements for crystallinity, porosity, or a certain chemical motif e.g. carboxylate groups. The definition was also comes very close to the self-definition of the words "metal", "organic", and "framework" that allow this of be understood and more or less correctly interpreted by a more general scientific audience, however, the broadness of the definition also capture the difficulty in standardising a nomenclature for a field as diverse in chemistry, rapidly growing, and mature as MOFs.

A popular nomenclature for MOFs that is more generally used describes MOFs as compounds which are constructed from secondary building units (SBUs) of metal nodes (usually metal ions or clusters) and organic linkers to form framework architectures which tend to be porous and crystalline. Such examples include the 


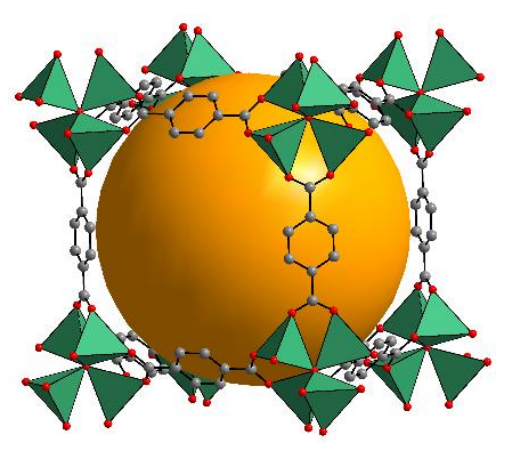

MOF-5

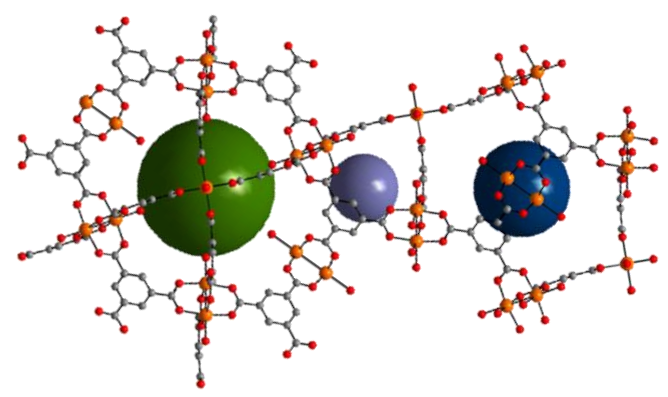

HKUST-1
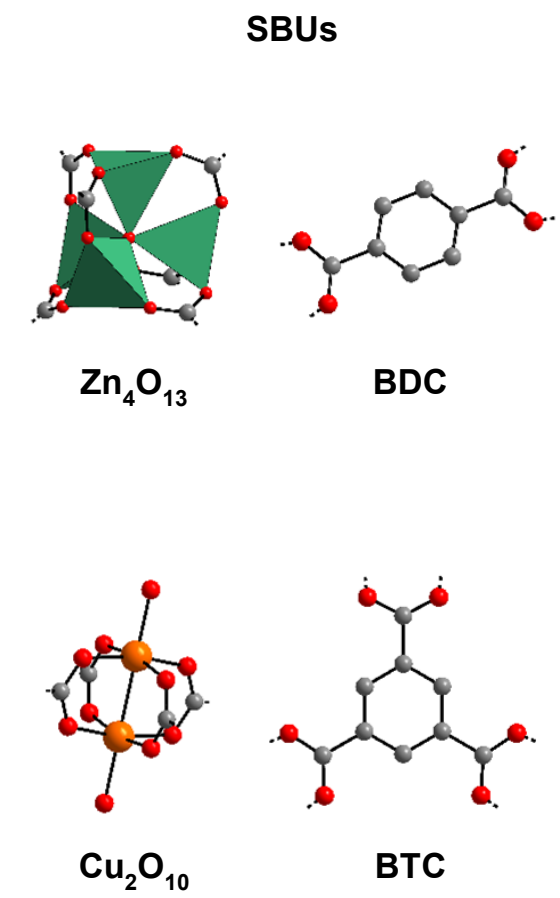

BTC

Figure 1.3 - MOF-5 and HKUST-1 with their respective SBUs. MOF-5 - $\mathrm{Zn}_{4} \mathrm{O}_{13}$ cluster and BDC linker, HKUST-1 - $\mathrm{Cu}_{2} \mathrm{O}_{10}, \mathrm{Cu}$ paddlewheel and BTC linker. Colour scheme: $\mathrm{ZnO}_{4}$ tetrahedra - green, $\mathrm{Cu}$ - orange, $\mathrm{O}$ - red, $\mathrm{C}$ - grey. $\mathrm{H}$ atoms are omitted for clarity. Golden, green, indigo, and blue spheres represent the different pore types present in HKUST-1 and MOF-5. They are centred in the void space and the radius is fixed so the sphere shown has equal volume of the void.

archetypal MOF-5 $\left(\left[\mathrm{Zn}_{4}(\mu 4-\mathrm{O})(\mathrm{BDC})_{3}\right], \mathrm{BDC}=\right.$ benzene-1,4-dicarboxylate $)$ and HKUST-1 $\left(\left[\mathrm{Cu}_{3}(\mathrm{BTC})_{2}\right]\right.$, BTC $=$ benzene-1,3,5-tricarboxylate $)$ where $\mathrm{Zn}$ and $\mathrm{Cu}$ clusters are connected by carboxylate linkers to form 3-dimensional porous frameworks, Figure 1.3. ${ }^{40,41}$

The wide variety of SBUs available and the different ways they can be combined lead to an ever-increasing number of MOFs, with varying degrees of porosity, network topologies and a rich chemical diversity. One approach to MOF synthesis is through reticular synthesis where carefully designed rigid molecular building blocks are assembled into predetermined structures. ${ }^{42}$ This leads to isoreticular chemistry where without changing the topology, the size of the framework pores are tuned in a systematic way, usually by changing the length/size and functionality of the ligands or SBU's. ${ }^{43}$ 
One functionality that can be added to certain MOFs and not seen in more traditional porous materials, such as zeolites, is a degree of flexibility. This has given rise to a subclass of MOFs dubbed "soft porous crystals". ${ }^{44}$ These MOFs can undergo significant structural changes such as massive expansions or contractions in volume and conformational changes when exposed to external stimuli such as temperature, light, pressure, and uptake of guest molecules into the pores. ${ }^{45}$

MOFs have been touted for many applications including catalysis, sensing, separations and gas storage. ${ }^{46} 47$ However, until recently an application had never been realised in a commercial setting. ${ }^{48}$ Under the name TruPick ${ }^{\mathrm{TM}}$, a MOF has been made to slowly release 1-methylcyclopropene (1-MCP) into storage containers for harvested fruit and vegetables. 1-MCP inhibits the production of ethylene by the fruit, a chemical that causes the food to ripen, thus significantly increasing storage times. This is a huge step forward for the field and promising for future industrial uses of MOFs.

\subsubsection{Porous Molecular Crystal (PMC)}

Porous molecular crystals (PMCs) are another class of porous material, however, unlike the framework materials previously discussed, PMCs are assembled from discrete molecules between which there are only non-covalent intermolecular interactions. PMCs can show features not achievable in framework materials. For example, by virtue of their molecular nature, they can be solution processed and have demonstrated advanced properties such as guest-responsive switchable porosity. ${ }^{49-52}$

PMCs are assembled from discrete building units by way of non-covalent interactions. The molecular components can be intrinsically porous (i.e. interconnected voids in molecular containers or cages). . A typical example of this would be porous cage like rigid molecules with windows that allow access to these permanent voids, Figure 1.4a. ${ }^{53}$ An alternative to intrinsic porosity is extrinsic porosity where the pore is in-between neighbouring molecules, Figure $1.4 \mathrm{~b} .{ }^{51}$ All PMCs are crystallised along with a solvent in the pores that must be removed in order to reveal the porosity. However, many structures collapse upon the removal of the guest solvent, leading to more dense or amorphous phases that are non- 
(a)

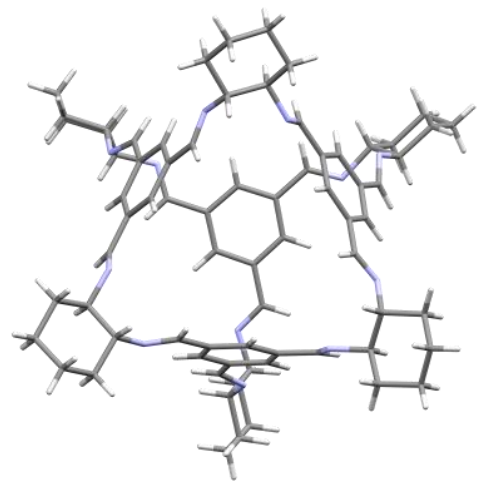

(b)

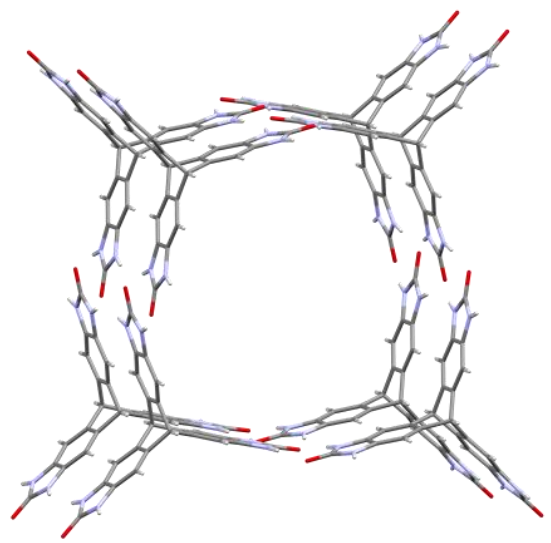

Figure 1.4 - (a) An example of a PMC with intrinsic porosity, 'Cage 3' from Cooper et al., 2009, a tetrahedral organic cage with void space contained within the molecule itself. (b) An example of extrinsic porosity triptycene trisbenzimidazolone (TTBI) from Mastalerz et al., 2012, where the porosity is between molecules.

porous. Molecular crystals that possess permanent micropores are considered rare due to the difficulty in predicting suitable molecular targets and the stability of the resultant PMCs. Novel examples of PMCs are often only serendipitously discovered, akin to locating 'a needle in a haystack'. ${ }^{54}$ However, recent advances in crystal structure prediction for the formation of PMCs are removing the guesswork from identifying potential synthetic targets. ${ }^{55-59}$

A further review of PMCs and their structure-function relationships is presented in Chapter 6 - The Effect of Pressure, Temperature and Chemical Stability of Phthalocyanine Porous Molecular Crystals. 


\subsection{The effect of pressure}

Mechanical loads can be applied to a material in several different ways; tension, compression, torsion, bending, shear, or combinations of these, Figure 1.5. ${ }^{60}$ However, these anisotropic stresses are not the subject of this work. Here we investigate crystalline materials under hydrostatic pressure where the forces are equally applied over the whole system resulting in an isotropic load.

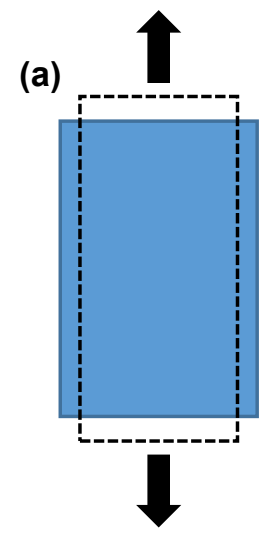

(d)

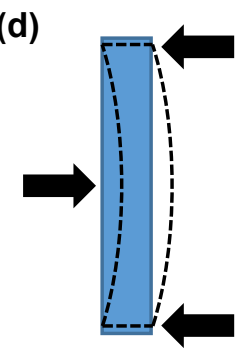

(b)

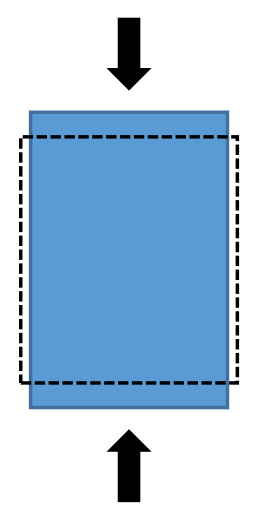

(e)

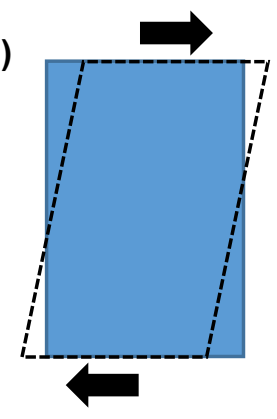

(c)
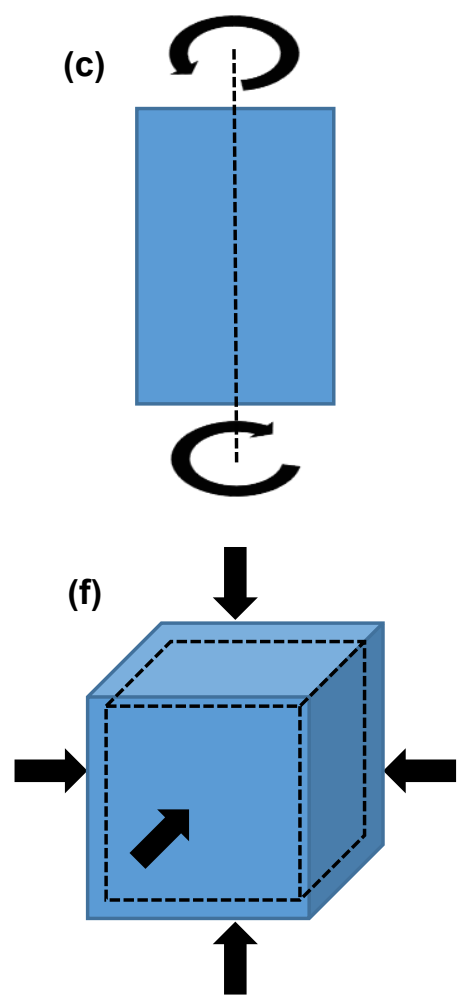

Figure 1.5 - Schematic representations of the common modes of mechanical compression: (a) uniaxial tension, (b) uniaxial compression, (c) torsion, (d) bending, (e) shear and (f) hydrostatic compression. Adapted from Tan et al. 2011.

The fundamental measure of the response of a crystal structure to the application of hydrostatic pressure at constant temperature is its isothermal bulk modulus $(K)$. This is the inverse of its compressibility and quantifies the variation of the volume $(V)$ of a solid with pressure $(P)$ at constant temperature $(T)$. This is defined as:

$$
K=-V\left(\frac{\partial P}{\partial V}\right)_{T}
$$


The bulk moduli for most organic and metal-organic materials fall in the range of $\sim 5$ to $50 \mathrm{GPa}$, and $\sim 25$ to $440 \mathrm{GPa}$ for inorganic solids. The higher the bulk moduli, the harder the solid is to compress. Some representative values for scale: $6.6 \mathrm{GPa}$ for $\mathrm{Ru}_{3}(\mathrm{CO})_{12}$, 13.1 GPa L-alanine, $25 \mathrm{GPa} \mathrm{NaCl}, 37 \mathrm{GPa}$ for quartz and $440 \mathrm{GPa}$ for diamond. ${ }^{61,62}$ They can be experimentally determined by measuring the volume variation with pressure, normally through diffraction, and fitting the resultant compression curve to an equation of state (EoS). Measured equations of state are usually parameterised by the bulk moduli along with the first and second pressure derivatives

$$
\begin{aligned}
K^{\prime} & =\left(\frac{\partial K}{\partial P}\right)_{T} \\
K^{\prime \prime} & =\left(\frac{\partial^{2} K}{\partial^{2} P}\right)_{T}
\end{aligned}
$$

There are many different EoS forms available, each with their own set of assumptions. The validity of these assumptions can only be determined by how well the EoS reproduces the experimental data. ${ }^{63}$ The Birch-Murnaghan EoS is a very successful and widely used EoS. ${ }^{64}$ This EoS uses the finite Eulerian definition of strain $(f)$, and the different orders of the EoS are derived by truncating the strain energy polynomial at various terms. The simplest of which is the $2^{\text {nd }}$ order form where the bulk modulus and pressure is expressed by:

$$
\begin{gathered}
K=K_{0}(1+2 f)^{5 / 2}(1+7 f) \\
P=3 K_{0}(1+2 f)^{5 / 2} f \\
\text { Where } f=\left[\left(V_{0} / V\right)^{2 / 3}-1\right] / 2
\end{gathered}
$$

Fortunately computer programs such as EoSFit from Angel et al. aid in the visualization and calculation of EoS however care must still be taken to fit the data with an appropriate EoS. ${ }^{65}$

Until now, we have assumed that the composition of the crystal does not change with pressure. While this holds for most dense materials, it is not the case for porous materials. If the molecular composition of the fluid that the crystal is compressed in, the pressure transmitting medium (PTM), is significantly larger 

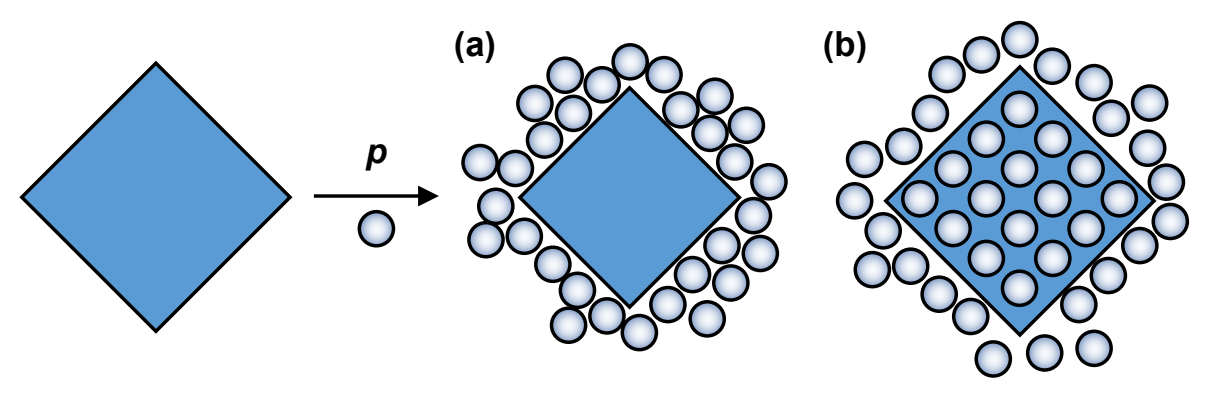

Figure 1.6 - Response to hydrostatic pressure in (a) non-penetrating and (b) penetrating medium.

than the pore widths of the open pores then the crystal will be directly compressed, and the medium is classed as non-penetrating, Figure 1.6a. Conversely, if the PTM is smaller than the pore width, it can act as a penetrating medium, entering the crystal and changing its composition, Figure 1.6b. Whether a PTM is penetrating or not is clearly a consequence of both the porous material and the PTM. The interplay between the host material and the PTM is a key part of this work. 


\subsection{Examples of the effects of pressure}

Over the last 10 years advances in the field of non-ambient crystallography has led to numerous studies at different conditions of temperature and pressure on MOFs, including gas sorption and high-pressure crystallographic studies. Both techniques have been the subject of comprehensive reviews, and here we briefly look at the effect of pressure and gas uptake on some archetypical MOFs, including HKUST-1, STAM-1, ZIF-8 and $\mathrm{Sc}_{2} \mathrm{BDC}_{3}$. ${ }^{66,67}$

The effect of pressure on zeolites will not be covered here. However, many of the pressure responses that will be described for MOFs also hold true for zeolites. ${ }^{68}$ Details of the practical techniques used for conducting high-pressure singlecrystal diffraction and in situ single crystal gas adsorption measurements are described in Chapter 2 - Methods.

\subsubsection{HKUST-1}

HKUST-1, also known as Cu-BTC, is constructed from $\mathrm{Cu}$ paddlewheel SBUs, $\mathrm{Cu}_{2} \mathrm{O}_{10}$, with benzene-1,3,5-tricarboxylate (BTC) linkers, Figure 1.3. Under ambient conditions, water is bound to the axial position of the Jahn-Teller distorted $\mathrm{Cu}$. This water was found to be labile and when removed leaves an open metal site that can play an important role in gas adsorption properties. In situ neutron and $\mathrm{X}$-ray diffraction studies have explored the uptake of gases such as $\mathrm{D}_{2}, \mathrm{CD}_{4}, \mathrm{CO}_{2}$, Ar, Xe and Kr. ${ }^{69-74}$ The neutron diffraction study by Peterson et al. revealed HKUST-1 to have 9 adsorption sites upon the adsorption of $\mathrm{D}_{2} \cdot{ }^{70}$ As the loading of $\mathrm{D}_{2}$ increased, the refined occupancies of the symmetry independent sites increased, demonstrating progressive filling of the adsorption sites. Gasses with a large dipole moment, such as $\mathrm{CO}_{2}$, interact with the open metal sites found in HKUST-1 through strong electrostatics; ${ }^{73}$ whereas noble gases favoured the small tetrahedral pockets (Figure 1.3 indigo sphere) over the open metal sites. ${ }^{73,74}$

High-pressure single-crystal (scXRD) and powder X-ray diffraction (pXRD) experiments have studied the effect of hydrostatic pressure on HKUST-1. ${ }^{75,76}$ Chapman et al. demonstrated that the compressibility of the MOF was strongly dependent on the molecular size of the PTM, Figure 1.7. On compression in the 
non-penetrating PTM FC-70, a bulky perfluorinated amine, HKUST-1 was directly compressed. With the smaller PTM isopropyl alcohol (IPA), and smaller still, a 16:3:1 methanol:ethanol:water mixture (MEW), there was a two-step compression. In the initial "hard" regime, $\mathrm{K} \approx 118 \mathrm{GPa}$, the pores of the framework superfill with the PTM, significantly reducing the compressibility. MEW and IPA transition at 2.2 and $0.8 \mathrm{GPa}$ respectively to a secondary "soft" regime, $\mathrm{K} \approx 30 \mathrm{GPa}$, where the PTM was forced out of the pores, increasing the compressibility. The pressures at which HKUST-1 becomes amorphous is also dependent on the size of the PTM. HKUST-1 retains its crystallinity until 7.5 GPa in MEW, 4.2 GPa in IPA, and only 2 GPa in FC-70.

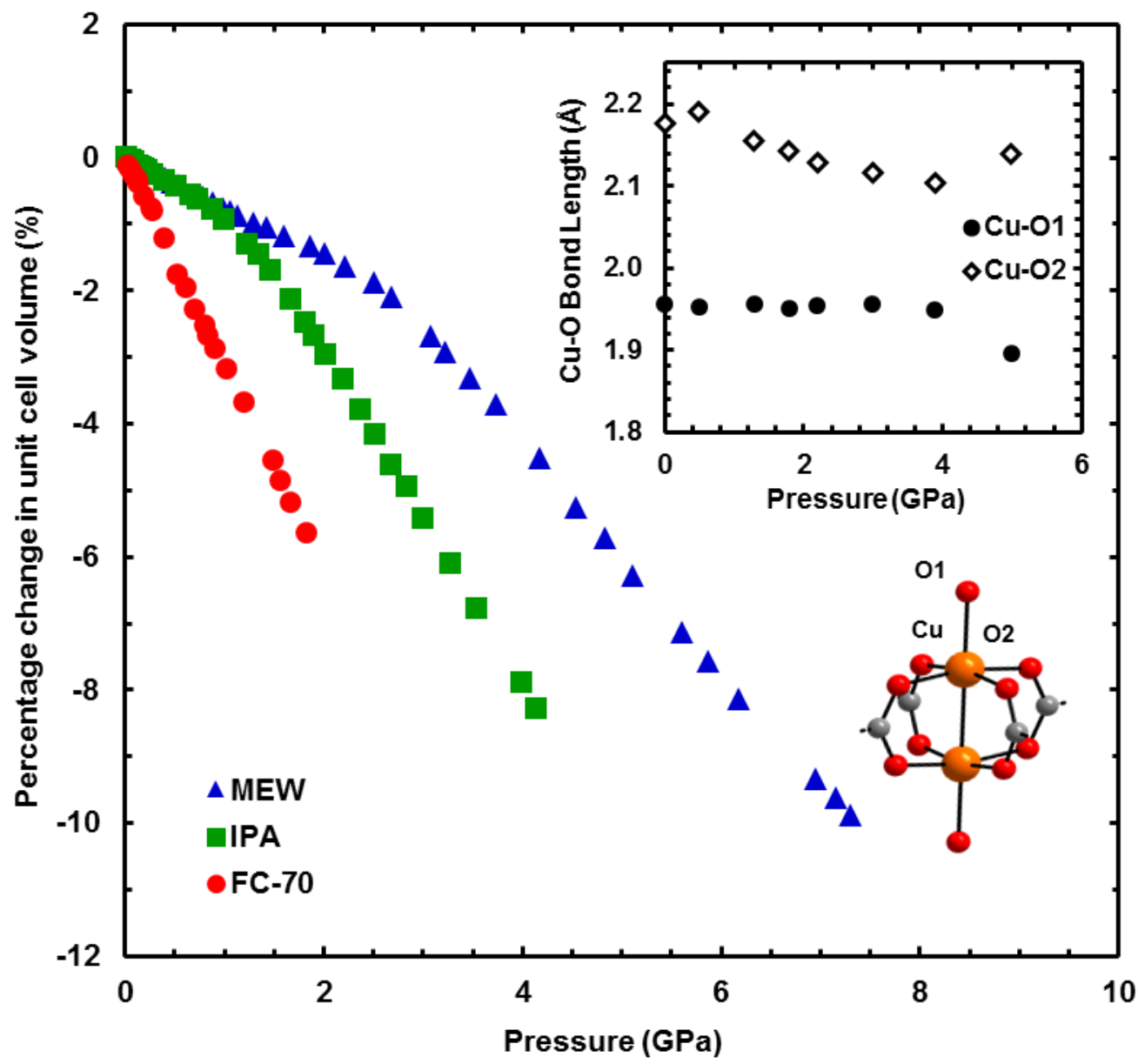

Figure 1.7 - Effect of different PTM on the compressibility of HKUST-1. Blue triangles, 16:3:1 methanol:ethanol:water mixture (MEW); green squares, isopropyl alcohol (IPA); red circles, FC-70, a bulky perfluorinated amine. Adapted from Chapman et al. Inset shows the change in $\mathrm{Cu}-\mathrm{O}$ bond lengths upon compression in $\mathrm{MEW}$. Close-up of the $\mathrm{Cu}$-paddlewheel representing the two different $\mathrm{Cu}-\mathrm{O}$ bond lengths measured by Graham et al. $\mathrm{Cu}$-atoms in brown, $\mathrm{O}$-atoms in red and $\mathrm{C}$-atoms in grey. $\mathrm{H}$ atoms are omitted for clarity. 
Since the high-pressure pXRD study by Chapman et al. did not report crystallographic refinements with atomistic data, Graham et al. carried out highpressure scXRD experiments with MEW to clarify the structural transitions between the hard and soft regions. ${ }^{76}$ From this experiment the calculated electron density in the pores was found to increase with increasing pressure during the hard regime, confirming the increased uptake of the PTM. Upon the transition to the soft regime, a sudden reduction of the equatorial $\mathrm{Cu}-\mathrm{O} 1$ distances and an elongation of the axial $\mathrm{Cu}-\mathrm{O} 2$ was observed, Figure 1.7. This indicated that the pore emptying mechanism was associated with the sudden compression of the shorter equatorial $\mathrm{Cu}-\mathrm{Ol}$ bonds. Compression of the $\mathrm{Cu}-\mathrm{Ol}$ bonds was also responsible for the reduction in volume during the hard to soft regime transition. This transition was observed at higher pressures in the single-crystal form (5 GPa) than in the powder $(\sim 2 \mathrm{GPa})$, which is attributed to a particle-size effect. This is known to exist in framework materials, most notably in the adsorption properties of ZIF-8. ${ }^{77}$ 


\subsubsection{STAM-1}

STAM-1, $\left[\mathrm{Cu}_{3} \mathrm{O}_{21} \mathrm{C}_{30} \mathrm{H}_{24}\right]_{n} .5 \mathrm{n}\left(\mathrm{H}_{2} \mathrm{O}\right)$, can be synthesized from the same starting materials as HKUST-1, $\mathrm{Cu}\left(\mathrm{NO}_{3}\right)_{2} .3 \mathrm{H}_{2} \mathrm{O}$ and benzene-1,3,5-tricarboxylic acid $\left(\mathrm{H}_{3} \mathrm{BTC}\right)$, but by using a 50:50 water:methanol mix in place of ethanol as the solvent during the solvothermal synthesis. ${ }^{78}$ During synthesis the $\mathrm{H}_{3} \mathrm{BTC}$ undergoes esterification, and upon crystallization, only monomethyl-esterified BTC ligands then link the same $\mathrm{Cu}$ paddlewheel nodes that are found in HKUST-1.

The resultant framework has a very different topology to HKUST-1, with STAM-1 forming a layered structure containing two channels types, hydrophilic and hydrophobic, both parallel to the crystallographic c-axis. The hydrophilic channels are lined with water molecules bound to the axial position of the $\mathrm{Cu}$ paddlewheel nodes, and the hydrophobic channels are lined by the ester groups of the monomethyl ester BTC linkers. Both channel types have a pore width of $\approx 4 \AA$ (Figure 1.8).

In situ single crystal experiments at ambient pressure showed that STAM-1 can undergo a single-crystal to single-crystal ligand exchange reaction, where the axial water bound to the $\mathrm{Cu}$ paddlewheel can be exchanged for methanol (referred to as STAM-1 $\left.\mathrm{MeOH}_{\mathrm{H}}\right) .{ }^{79}$ This caused the once open hydrophilic channels to become isolated hydrophobic pores, due to the penetration of methyl groups into the channel (Figure 1.8).

This is an example of post-synthetic modification (PSM), defined simply as the chemical modification of a framework after it has been synthesized. ${ }^{80}$ PSM in the MOF community is a field in its own right, and has been the subject of numerous reviews. ${ }^{81-83}$ PSM allows the synthesis of MOFs which are impossible to make via direct synthetic approaches, opening up new avenues of chemistry and potential applications for MOFs. 
(a)
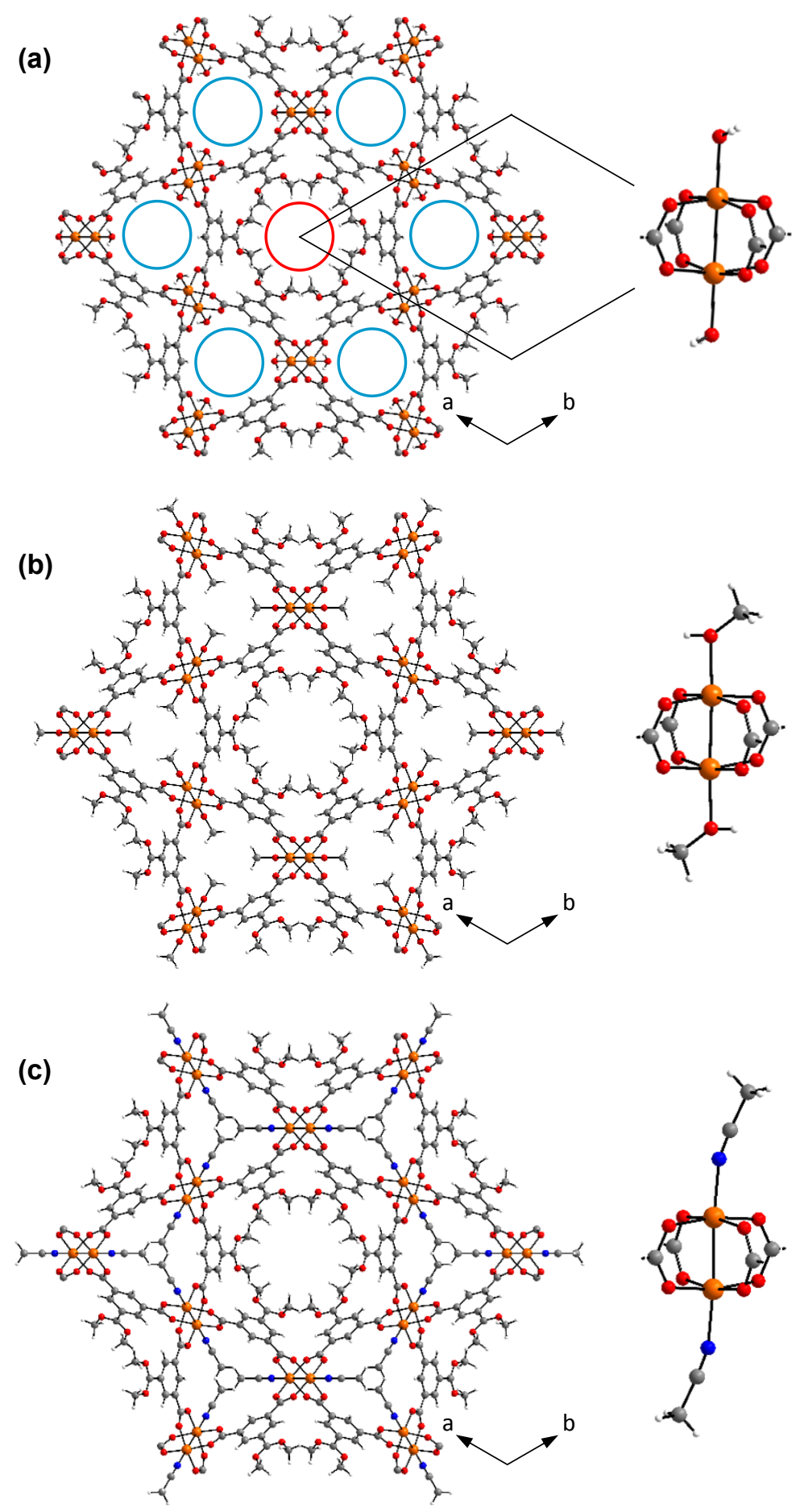

Figure 1.8 - Packing diagrams as viewed parallel to the $c$-axis with central hydrophobic pore (red circle) surrounded by hydrophilic pores (blue circles) and paddlewheel units of STAM-1 (a), STAM-1 $1_{\mathrm{MeOH}}(\mathrm{b})$, and STAM-1 $\mathrm{l}_{\mathrm{MeCN}}(\mathrm{c})$. For STAM-1 $\mathrm{l}_{\mathrm{MeCN}}$ the three possible coordination sites of $\mathrm{MeCN}$ are shown with $1 / 3^{\text {rd }}$ occupancy. 
McKellar et al. went on to investigate STAM-1 at high-pressure with various PTMs including methanol, ethanol, acetonitrile, and acetaldehyde. With methanol, STAM-1 was stable to $5.7 \mathrm{GPa}$ as methanol fills the small hydrophilic channel, exchanging with the axial $\mathrm{H}_{2} \mathrm{O}$, and superfilling the large hydrophobic channel. However, upon immersion in ethanol at ambient pressure the crystal rapidly disintegrated becoming polycrystalline. The breakdown of the crystal was attributed to a strain-induced collapse of the framework caused by the exchange of the axial water for the sterically larger ethanol, which has a molecular volume $\approx 42 \AA^{3}$. This was confirmed on compression in the similarly sized acetaldehyde $\left(\sim 44 \AA^{3}\right)$ where uptake into the pores was observed, yet no exchange took place. STAM-1 was stable up to $5.1 \mathrm{GPa}$ in acetaldehyde.

For acetonitrile (which has a molecular volume of $\sim 43 \AA^{3}$ ) no exchange was observed at ambient pressure, however, upon loading at $0.3 \mathrm{GPa}$, the exchange was observed. The crystal does not undergo the strain-induced collapse observed with ethanol as the acetonitrile only exchanged for $1 / 3$ of the axial $\mathrm{H}_{2} \mathrm{O}$ ligands, suggesting distinct differences in the $\mathrm{Cu}$ affinity for ethanol and acetonitrile. This was the first example of a PSM of a MOF at pressure, demonstrating how the application of pressure is a useful tool to induce PSM and thus facilitate discovery of new framework materials. ${ }^{79}$ 


\subsubsection{ZIF-8}

The zeolitic imidazolate framework ZIF-8 has a sodalite (SOD) topology formed from $\mathrm{Zn}$ nodes and 2-methylimidazolate (MeIm) linkers, [Zn(MeIm) $)_{2}$. The structure contains large cavities ( $11.6 \AA$ in diameter) that are interconnected through 6-ring windows which measure $\sim 3.40 \AA$ and 4-ring windows that under ambient conditions are closed. Gas adsorption has been experimentally reported for $\mathrm{D}_{2}, \mathrm{CD}_{4}$ and $\mathrm{N}_{2}$ despite both $\mathrm{CD}_{4}$ and $\mathrm{N}_{2}$ being theoretically too large to pass through the 6-ring windows of the framework, with kinetic diameters of $3.80 \AA$ and $3.60 \AA$ respectively. ${ }^{10,84-87}$ The fact that both $\mathrm{CD}_{4}$ and $\mathrm{N}_{2}$ are adsorbed into ZIF-8 indicates the framework must have a certain degree of flexibility around the channel openings.

scXRD was used to investigate the behaviour of ZIF-8 under high-pressure. ${ }^{88} \mathrm{~A}$ 4:1 methanol:ethanol mix was used as the PTM and upon increasing pressure, the PTM was observed entering the channels, initially causing swelling of the framework to $0.18 \mathrm{GPa}$. By $1.47 \mathrm{GPa}$, a single-crystal phase transition took place, in which the MeIm linkers rotated by $\sim 25^{\circ}$, hereafter referred to as ZIF-8HP (Figure 1.5). This transition increased the diameter of the 6-ring windows significantly and opened a second channel interconnecting the large cavities through the smaller 4-ring windows. The larger pore volume of ZIF-8HP allowed for further uptake of guest molecules and re-inflation of the framework. The structural change was found to be reversible after decreasing the pressure.

Previous adsorption studies under ambient conditions had revealed a sigmoidal shape for the $\mathrm{N}_{2}$ adsorption isotherm for ZIF-8 at $77 \mathrm{~K}$, and indicated a potential change in the pore structure on the uptake of guest species. GCMC simulations showed that the ZIF-8HP structure obtained at 1.47 GPa could be used to model the $\mathrm{N}_{2}$ uptake after the step in the isotherm. This was confirmed by in situ pXRD studies conducted at $77 \mathrm{~K}$ where $\mathrm{N}_{2}$ loaded ZIF-8 (collected at 0.4 bar) showed the same reorientation of the imidazolate linkers as found in ZIF-8HP. ${ }^{86}$ 


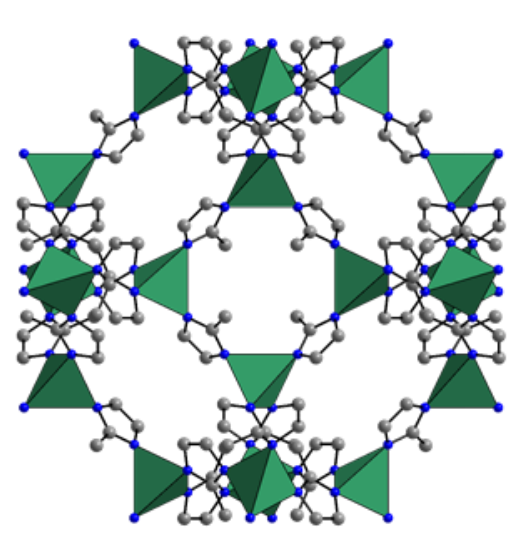

Ambient pressure

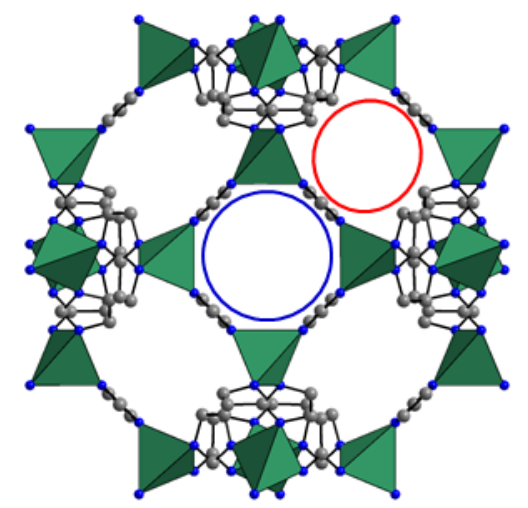

$1.47 \mathrm{GPa}$

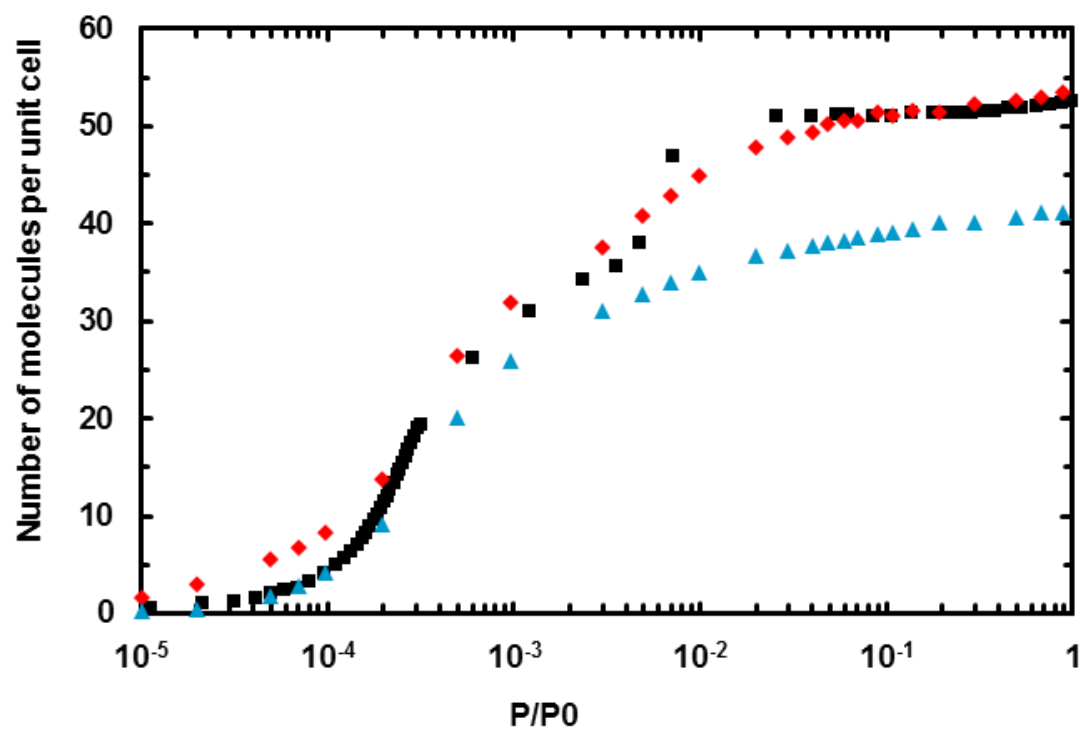

Figure 1.9 - Representation of the phase change adopted by ZIF-8 at 1.47 GPa in ethanol, leading to the channels to open through the rotation of the MeIm linkers. $\mathrm{ZnN}_{4}$ tetrahedra are represented in green, $\mathrm{N}$-atoms in blue and $\mathrm{C}$-atoms in grey. $\mathrm{H}$-atoms are omitted for clarity. 4-ring and 6-ring windows are indicated by blue and red circles respectively. The calculated adsorption isotherms using both the ambient pressure model (light blue triangles) and the high-pressure model (red diamonds), in comparison to the experimentally measured isotherm (black squares). 


\subsection{4 $\mathrm{Sc}_{2}(\mathrm{BDC})_{3}$}

$\mathrm{Sc}_{2} \mathrm{BDC}_{3}$ is a scandium terephthalate $\mathrm{MOF}$ formed from $\mathrm{ScO}_{6}$ octahedral nodes linked by 1,4-benzenedicarboxylate (BDC) linkers. This forms a chemically and thermally stable small pore MOF that crystallises in the orthorhombic space group Fddd under ambient conditions. ${ }^{89}$ Parallel to the $a$-axis are small triangular channels with a free diameter of $\sim 4 \AA$ that are hydrophobic in nature. Under ambient conditions, there are two crystallographically unique BDC linkers designated as Group 1 and 2, Figure 1.10. There are small gaps in the channel walls between adjacent BDC ligands linking the triangular shaped pores, giving rise to porosity in three-dimensions.

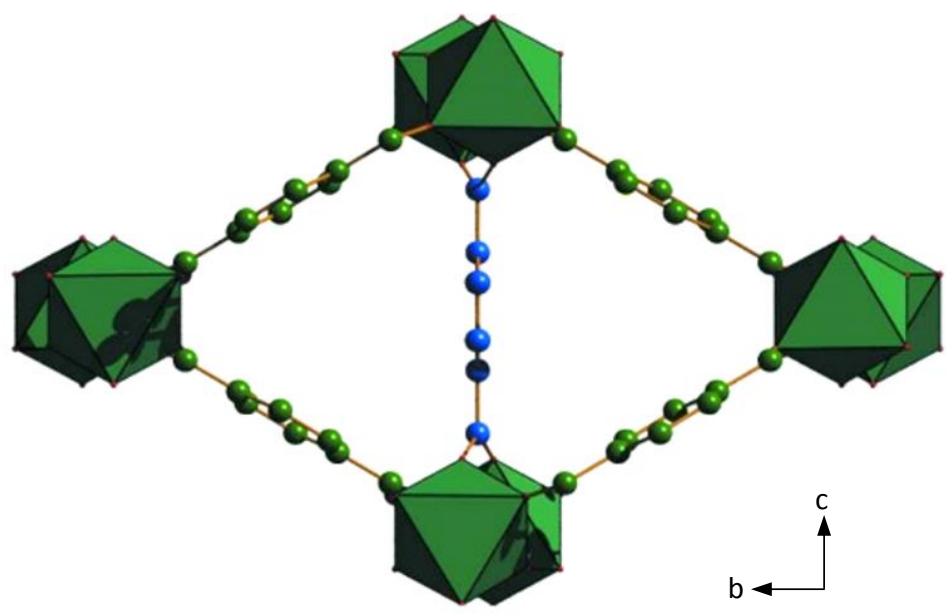

Figure 1.10 - The structure of $\mathrm{Sc}_{2} \mathrm{BDC}_{3}$ at ambient conditions as viewed parallel to the $a$-axis. The two crystallographically unique BDC linkers Group 1 and 2, coloured green and blue, respectively. Figure adapted from Graham et al. (2014)

In situ gas adsorption diffraction studies on $\mathrm{Sc}_{2} \mathrm{BDC}_{3}$ at low temperatures $(\sim 230$ $\mathrm{K}$ ) allowed the adsorption sites of small gas molecules such as $\mathrm{CO}_{2}$ and light hydrocarbons physisorbed in the pores to be located. ${ }^{90,91}$ Upon the uptake of $\mathrm{CO}_{2}$ the structure underwent a displacive phase transition from $F d d d$ to $C 2 / c$ to better coordinate the adsorbate molecules. The small size of the pores and packing of adsorbate molecules made it possible to determine the location of the guests accurately by diffraction. 
The nitro modification of $\mathrm{Sc}_{2} \mathrm{BDC}_{3}, \mathrm{Sc}_{2}\left(\mathrm{NO}_{2}-\mathrm{BDC}\right)_{3}$ has also been investigated. Topologically $\mathrm{Sc}_{2}\left(\mathrm{NO}_{2}-\mathrm{BDC}\right)_{3}$ is very similar to $\mathrm{Sc}_{2} \mathrm{BDC}_{3}$, however, to accommodate the bulky nitro groups the framework distorts to the previously observed $C 2 / c$ phase under ambient conditions. The nitro groups block the channels to $\mathrm{N}_{2}$ uptake at $77 \mathrm{~K}$ but permit some uptake of $\mathrm{CO}_{2}$ at $273 \mathrm{~K}$, and a greater uptake of $\mathrm{CO}_{2}$ at $298 \mathrm{~K}$ compared to $\mathrm{Sc}_{2} \mathrm{BDC}_{3}$. ${ }^{90}$

Graham et al. characterized the high-pressure behaviour of both $\mathrm{Sc}_{2} \mathrm{BDC}_{3}$ and $\mathrm{Sc}_{2}\left(\mathrm{NO}_{2}-\mathrm{BDC}\right)_{3}$ through high-pressure scXRD. ${ }^{92}$ Using methanol as a penetrating $\mathrm{PTM}$, molecules superfill the pores of $\mathrm{Sc}_{2} \mathrm{BDC}_{3}$ leading to an initial swelling of the unit cell. Two distinct adsorption sites (referred to as Site 1 and Site 2) were located on either side of the gaps created by the two symmetry-independent BDC linkers. The occupancy of the methanol at each site increased with increasing pressure leading to Site 1 being fully occupied by $0.3 \mathrm{GPa}$, while Site 2 reached $80 \%$ occupancy by 1.1 GPa, Figure 1.11Figure 1.12. As previously discussed for HKUST-1, the inclusion of guest molecules into the framework delays the onset of amorphisation. In methanol, $\mathrm{Sc}_{2} \mathrm{BDC}_{3}$ remains crystalline until above 3.0 GPa, whereas, on using the non-penetrating PTM Fluorinert (FC-77), $\mathrm{Sc}_{2} \mathrm{BDC}_{3}$ was directly compressed and above $0.14 \mathrm{GPa}$ undergoes a fully reversible crystalline-amorphous phase transition.

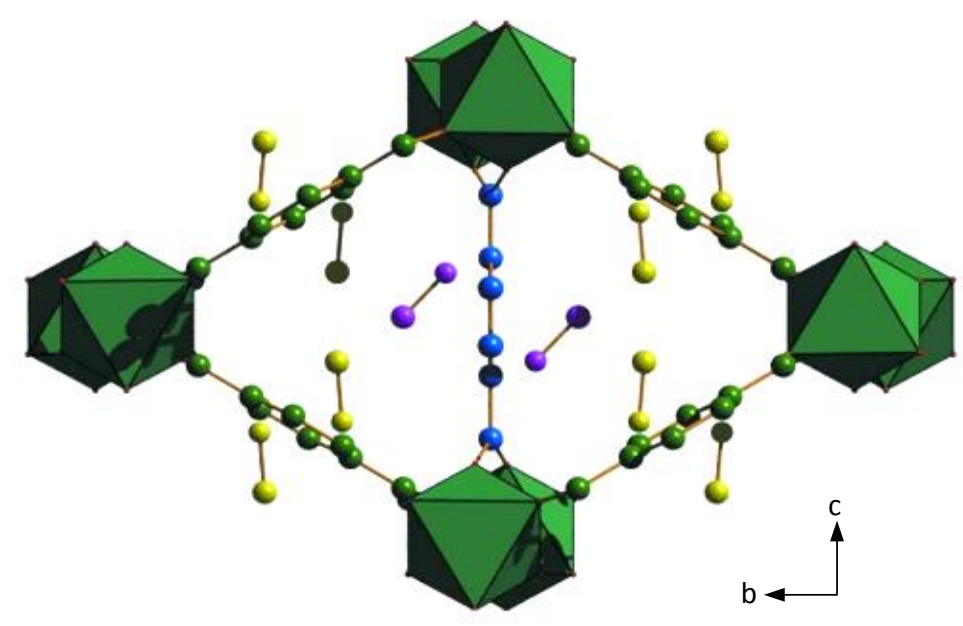

Figure $1.11-\mathrm{Sc}_{2} \mathrm{BDC}_{3}$ at $1.1 \mathrm{GPa}$ in methanol with the adsorption Site 1 (purple) and Site 2 (yellow) shown located on either side of the gaps created by the two symmetryindependent BDC linkers. Site 1 is fully occupied while Site 2 is $80 \%$ occupied. Figure adapted from Graham et al. (2014). 
On direct compression of the nitro-derivatised $\mathrm{Sc}_{2}\left(\mathrm{NO}_{2}-\mathrm{BDC}\right)_{3}$ in FC-77 from ambient pressure to $0.8 \mathrm{GPa}$, a single-crystal to single-crystal phase transition $(C 2 / c$ to $F d d 2)$ via a rotation of the $\mathrm{ScO}_{6}$ octahedra and an associated distortion of the porous channels was observed. On increasing pressure further, the crystalline phases of $\mathrm{Sc}_{2}\left(\mathrm{NO}_{2}-\mathrm{BDC}\right)_{3}$ displayed a significant increase in stability compared to the unfunctionalized $\mathrm{Sc}_{2} \mathrm{BDC}_{3}$, with $\mathrm{Sc}_{2}\left(\mathrm{NO}_{2}-\mathrm{BDC}\right)_{3}$ becoming amorphous above $2.6 \mathrm{GPa}$.
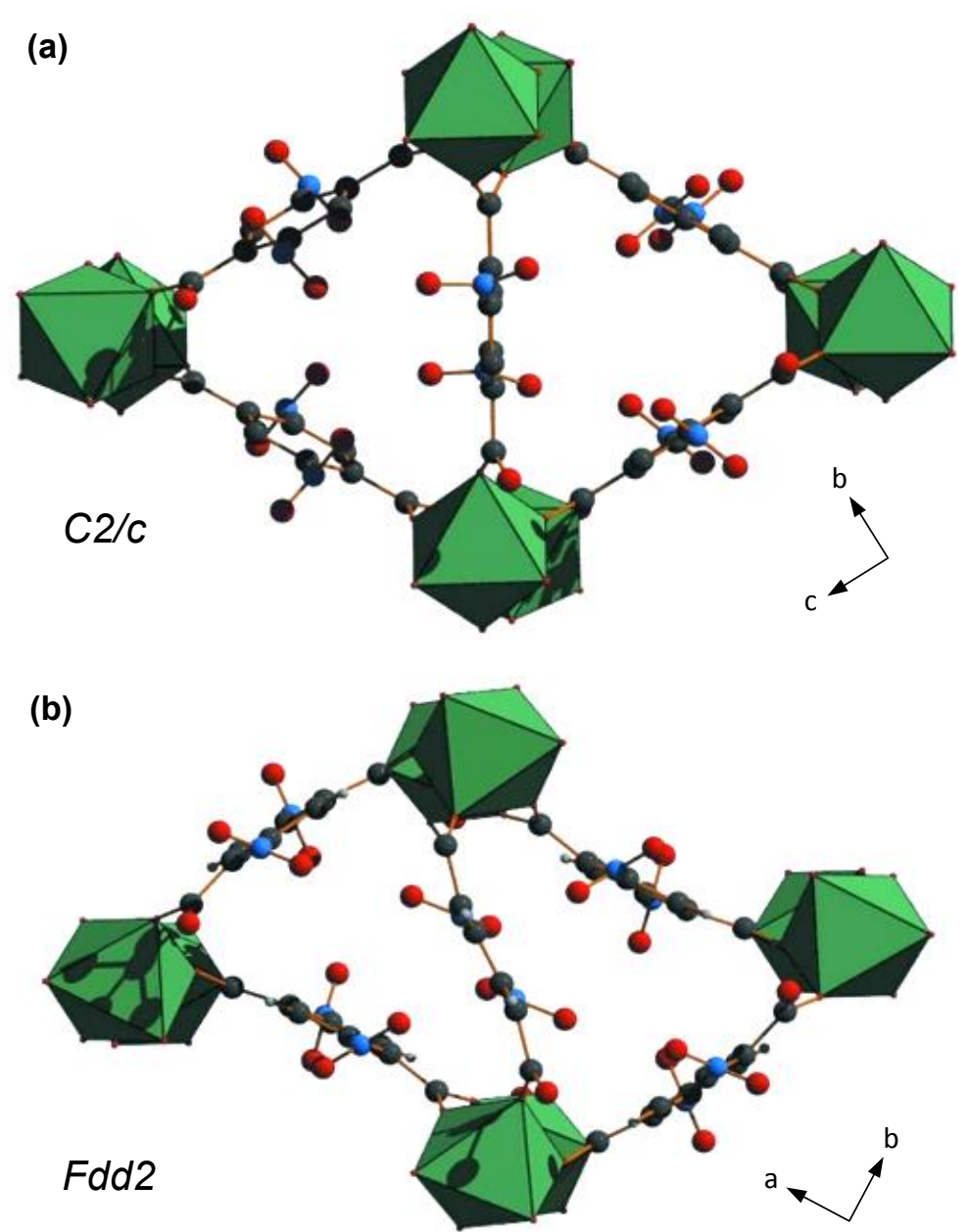

Figure $1.12-\mathrm{Sc}_{2}\left(\mathrm{NO}_{2}-\mathrm{BDC}\right)_{3}$ at (a) ambient pressure and (b) $0.8 \mathrm{GPa}$ on direct compression in $\mathrm{FC}-77$. Note, the $25^{\circ}$ tilt of the $\mathrm{ScO}_{6}$ octahedra in the high pressure phase resulting in the collapse of the porous network. Colour scheme: $\mathrm{O}$, red; $\mathrm{C}$, grey; $\mathrm{N}$, blue and $\mathrm{ScO} 6$ octahedra, green. $\mathrm{H}$ atoms have been removed for clarity. Figure adapted from Graham et al. (2014) 
There was no initial swelling from superfilling on compression of $\mathrm{Sc}_{2}\left(\mathrm{NO}_{2}-\mathrm{BDC}\right)_{3}$ in methanol suggesting that methanol uptake was much lower than for $\mathrm{Sc}_{2} \mathrm{BDC}_{3}$, due to the presence of the bulky $\mathrm{NO}_{2}$ side groups. On increasing pressure, the unit cell compressed steadily until amorphisation took place above $3.3 \mathrm{GPa}$.

The very initial swelling behaviour and structural response of $\mathrm{Sc}_{2} \mathrm{BDC}_{3}$ upon the uptake of branched and unbranched hydrocarbons will be presented in Chapter 4 .

\subsubsection{Overview}

Presented here are only a few of the many studies on a wide range of MOFs chosen to highlight some of the key effects observed with pressure on porous materials. It is clear that the PTM that a sample is compressed in plays a pivotal role in how the framework material responds to pressure. This can massively effect compression behaviour, phase behaviour, and amorphisation pressure. Linked to this is pressure induced superfilling of the pores, and the initial swelling of materials on the uptake of guest molecules when compressed in a penetrating PTM. Changing the content of the pores can lead to PSM, as seen with STAM-1. These highlighted studies also show how the flexibility of MOFs can enhance the selectivity and uptake properties of porous materials, and that high-pressure techniques have proven to be pivotal in understanding guest uptake, mechanical stability and phase behaviour in MOFs, as well as access new MOF structures via high-pressure PSM. 


\subsection{Outline of thesis}

In this thesis, we present the effect of pressure on porous materials at a range of pressure regions using in situ diffraction methods. As part of these studies, we have developed new methods for investigating porous materials at much more moderate pressures, up to 1500 bar, a pressure region that is relatively unexplored, and yet vital for understanding pressure induced behaviour below the pressures attainable in a diamond anvil cell (DAC). Here, we have investigated the effect of pressure on the porous framework $\mathrm{Sc}_{2} \mathrm{BDC}_{3}$ where the uptake and exchange of guest, framework swelling, and breathing modes of this flexible system are all observed up to 1200 bar.

We have also studied the effect of pressure on a $\mathrm{Cu}$ based framework using traditional DAC high-pressure methods. This framework displays an unusual number of pressure induced phenomena including phase transitions, colour changes and PTM dependent Jahn-Teller switching.

Further pushing the boundaries of DAC experiments, we go on to present the first high-pressure diffraction experiment on a porous molecular crystal structure. This fullerene stabilised cobalt phthalocyanine cocrystal exhibits remarkable stability to hydrostatic compression, along with exceptional chemical and thermal stability. Following this, we finish with in-situ gas sorption studies on this porous molecular crystal investigating the uptake, and chemisorption of gasses to the open metal site. 


\subsection{Aims and Objectives}

Chapter 3 covers the design, construction and method development for a new pressure cell for the small molecule beamline I19 at the Diamond Light Source. At the outset a list of aims and objectives for this cell were outlined:

- To enable single-crystal diffraction from ambient pressure to more than 1000 bar regularly with very precise pressure control (ca. 10 bar) both on increasing and decreasing pressure.

- To have little or no restriction to available reciprocal space to allow the study of low symmetry systems.

- As a user facility, the cell would have to be simple, reliable, robust, and safe for all users.

- To integrate this set up with the existing instrumentation on beamline I19.

Chapter 4 follows on from the development and initial testing of the sapphire capillary pressure cell, and makes use of this cell to investigate the effect of pressure on a small pore $\mathrm{MOF}\left(\mathrm{Sc}_{2} \mathrm{BDC}_{3}\right)$. Here the aims and objectives were to:

- Investigate the structural behaviour of $\mathrm{Sc}_{2} \mathrm{BDC}_{3}$ when exposed to $n$-pentane and isopentane up to 1200 bar.

- Map pressure induced phenomena found upon the initial compression of porous materials, such as superfilling and swelling of the structure.

- Complement and expand upon previous high pressure measurements (DAC).

The aims and objectives for Chapter 5 were to investigate the effect of pressure on a novel $\mathrm{Cu}$ based framework using more conventional DAC high-pressure techniques adapted for work on porous materials. These include:

- Investigation using various pressure transmitting medium

- Visual monitoring of the sample at pressure.

- The tracking of pressure induced structural changes. 
Chapters 6 and 7 present a Porous Molecular Crystal (PMC) system that is formed from the simple co-crystallisation of (dipPhO $)_{8} P c C o$ and a fullerene $\left(\mathrm{C}_{60}\right.$ or $\left.\mathrm{C}_{70}\right)$. The aims and objectives were to:

- Present the remarkable chemical and thermal stability of these systems.

- Perform the first high-pressure diffraction investigation of a PMC.

- Investigate the effects of high-pressure on these systems and how the choice of $\mathrm{C}_{60}$ or $\mathrm{C}_{70}$ influences these.

- Investigate, via in-situ X-ray diffraction, the stability of these PMC to highvacuum and study the gas sorption on exposure to $\mathrm{O}_{2}$ and $\mathrm{CO}$. 


\subsection{References}

1 Rouquerol, J., Avnir, D., Fairbridge, C. W., Everett, D. H., Haynes, J. M., Pernicone, N., Ramsay, J. D. F., Sing, K. S. W. \& Unger, K. K. Recommendations for the characterization of porous solids (Technical Report). Pure and Applied Chemistry 66, 1739-1758, doi:10.1351/pac199466081739 (1994).

2 Zdravkov, B., Čermák, J., Šefara, M. \& Janků, J. Pore classification in the characterization of porous materials: A perspective. Central European Journal of Chemistry 5, 385-395, doi:10.2478/s11532-007-0017-9 (2007).

3 Lee, J., Farha, O. K., Roberts, J., Scheidt, K. A., Nguyen, S. T. \& Hupp, J. T. Metal-organic framework materials as catalysts. Chemical Society Reviews 38, 1450-1459, doi:10.1039/b807080f (2009).

4 Li, H., Pan, Q., Ma, Y., Guan, X., Xue, M., Fang, Q., Yan, Y., Valtchev, V. \& Qiu, S. Three-Dimensional Covalent Organic Frameworks with Dual Linkages for Bifunctional Cascade Catalysis. Journal of the American Chemical Society 138, 14783-14788, doi:10.1021/jacs.6b09563 (2016).

5 Wang, X., Han, X., Zhang, J., Wu, X., Liu, Y. \& Cui, Y. Homochiral 2D Porous Covalent Organic Frameworks for Heterogeneous Asymmetric Catalysis. Journal of the American Chemical Society 138, 12332-12335, doi:10.1021/jacs.6b07714 (2016).

6 Lin, X., Jia, J., Hubberstey, P., Schroeder, M. \& Champness, N. R. Hydrogen storage in metal-organic frameworks. Crystengcomm 9, 438-448, doi:10.1039/b706207a (2007).

7 Llewellyn, P. L., Bourrelly, S., Serre, C., Vimont, A., Daturi, M., Hamon, L., De Weireld, G., Chang, J.-S., Hong, D.-Y., Hwang, Y. K., Jhung, S. H. \& Ferey, G. High uptakes of $\mathrm{CO}_{2}$ and $\mathrm{CH}_{4}$ in mesoporous metal-organic frameworks MIL-100 and MIL-101. Langmuir 24, 7245-7250, doi:10.1021/la800227x (2008).

8 Nugent, P., Belmabkhout, Y., Burd, S. D., Cairns, A. J., Luebke, R., Forrest, K., Pham, T., Ma, S., Space, B., Wojtas, L., Eddaoudi, M. \& Zaworotko, M. J. Porous materials with optimal adsorption thermodynamics and kinetics for CO2 separation. Nature 495, 80-84, doi:10.1038/naturel1893 (2013). 
9 Sumida, K., Rogow, D. L., Mason, J. A., McDonald, T. M., Bloch, E. D., Herm, Z. R., Bae, T.-H. \& Long, J. R. Carbon Dioxide Capture in MetalOrganic Frameworks. Chemical Reviews 112, 724-781, doi:10.1021/cr2003272 (2012).

10 Yang, S., Sun, J., Ramirez-Cuesta, A. J., Callear, S. K., David, W. I. F., Anderson, D. P., Newby, R., Blake, A. J., Parker, J. E., Tang, C. C. \& Schröder, M. Selectivity and direct visualization of carbon dioxide and sulfur dioxide in a decorated porous host. Nature Chemistry, 4, 887-894, doi:10.1038/nchem.1457 (2012).

11 Ma, H., Ren, H., Meng, S., Yan, Z., Zhao, H., Sun, F. \& Zhu, G. A 3D microporous covalent organic framework with exceedingly high $\mathrm{C}_{3} \mathrm{H}_{8} / \mathrm{CH}_{4}$ and $\mathrm{C}_{2}$ hydrocarbon/ $\mathrm{CH}_{4}$ selectivity. Chemical Communications 49, 97739775, doi:10.1039/C3CC45217D (2013).

12 Pramudya, Y. \& Mendoza-Cortes, J. L. Design Principles for High $\mathrm{H}_{2}$ Storage Using Chelation of Abundant Transition Metals in Covalent Organic Frameworks for 0-700 bar at 298 K. Journal of the American Chemical Society 138, 15204-15213, doi:10.1021/jacs.6b08803 (2016).

13 Cychosz, K. A., Ahmad, R. \& Matzger, A. J. Liquid phase separations by crystalline microporous coordination polymers. Chemical Science 1, 293302, doi:10.1039/c0sc00144a (2010).

14 Herm, Z. R., Swisher, J. A., Smit, B., Krishna, R. \& Long, J. R. Metal-Organic Frameworks as Adsorbents for Hydrogen Purification and Precombustion Carbon Dioxide Capture. Journal of the American Chemical Society 133, 5664-5667, doi:10.1021/jall1411q (2011).

15 Lu, G. \& Hupp, J. T. Metal-Organic Frameworks as Sensors: A ZIF-8 Based Fabry-Perot Device as a Selective Sensor for Chemical Vapors and Gases. Journal of the American Chemical Society 132, 7832-7833, doi:10.1021/jal01415b (2010).

16 Horcajada, P., Chalati, T., Serre, C., Gillet, B., Sebrie, C., Baati, T., Eubank, J. F., Heurtaux, D., Clayette, P., Kreuz, C., Chang, J. S., Hwang, Y. K., Marsaud, V., Bories, P. N., Cynober, L., Gil, S., Ferey, G., Couvreur, P. \& Gref, R. Porous metal-organic-framework nanoscale carriers as a potential 
platform for drug delivery and imaging. Nature Materials 9, 172-178, doi:10.1038/nmat2608 (2010).

17 Bai, L., Phua, S. Z. F., Lim, W. Q., Jana, A., Luo, Z., Tham, H. P., Zhao, L., Gao, Q. \& Zhao, Y. Nanoscale covalent organic frameworks as smart carriers for drug delivery. Chemical Communications 52, 4128-4131, doi:10.1039/C6CC00853D (2016).

18 Vyas, V. S., Vishwakarma, M., Moudrakovski, I., Haase, F., Savasci, G., Ochsenfeld, C., Spatz, J. P. \& Lotsch, B. V. Exploiting Noncovalent Interactions in an Imine-Based Covalent Organic Framework for Quercetin Delivery. Advanced Materials 28, 8749-8754, doi:10.1002/adma.201603006 (2016).

19 Allendorf, M. D., Bauer, C. A., Bhakta, R. K. \& Houk, R. J. T. Luminescent metal-organic frameworks. Chemical Society Reviews 38, 1330-1352, doi:10.1039/b802352m (2009).

20 Blake, A. J., Champness, N. R., Easun, T. L., Allan, D. R., Nowell, H., George, M. W., Jia, J. \& Sun, X.-Z. Photoreactivity examined through incorporation in metal-organic frameworks. Nature Chemistry, 2, 688-694, doi:10.1038/nchem.681 (2010).

21 Kreno, L. E., Leong, K., Farha, O. K., Allendorf, M., Van Duyne, R. P. \& Hupp, J. T. Metal-Organic Framework Materials as Chemical Sensors. Chemical Reviews 112, 1105-1125, doi:10.1021/cr200324t (2012).

22 Lu, Z.-Z., Zhang, R., Li, Y.-Z., Guo, Z.-J. \& Zheng, H.-G. Solvatochromic Behavior of a Nanotubular Metal-Organic Framework for Sensing Small Molecules. Journal of the American Chemical Society 133, 4172-4174, doi:10.1021/ja109437d (2011).

23 Ding, S.-Y., Dong, M., Wang, Y.-W., Chen, Y.-T., Wang, H.-Z., Su, C.-Y. \& Wang, W. Thioether-Based Fluorescent Covalent Organic Framework for Selective Detection and Facile Removal of Mercury(II). Journal of the American Chemical Society 138, 3031-3037, doi:10.1021/jacs.5bl0754 (2016).

24 Babaei, H., McGaughey, A. J. H. \& Wilmer, C. E. Effect of pore size and shape on the thermal conductivity of metal-organic frameworks. Chemical Science 8, 583-589, doi:10.1039/C6SC03704F (2017). 
25 Hurd, J. A., Vaidhyanathan, R., Thangadurai, V., Ratcliffe, C. I., Moudrakovski, I. L. \& Shimizu, G. K. H. Anhydrous proton conduction at $150{ }^{\circ} \mathrm{C}$ in a crystalline metal-organic framework. Nature Chemistry, 1, 705710, doi:10.1038/nchem.402 (2009).

$26 \mathrm{Xu}, \mathrm{H} .$, Tao, S. \& Jiang, D. Proton conduction in crystalline and porous covalent organic frameworks. Nature Materials 15, 722-726, doi:10.1038/nmat4611 (2016).

27 Kurmoo, M. Magnetic metal-organic frameworks. Chemical Society Reviews 38, 1353-1379, doi:10.1039/b804757j (2009).

28 Hu, Z., Deibert, B. J. \& Li, J. Luminescent metal-organic frameworks for chemical sensing and explosive detection. Chemical Society Reviews 43, 5815-5840, doi:10.1039/C4CS00010B (2014).

29 Flanagan, D. M. Zeolites [Advance Release]. U.S. Geological Survey Minerals Yearbook, 84.81-84.85 (2015).

30 Jha, B. \& Singh, D. N. in Fly Ash Zeolites: Innovations, Applications, and Directions 5-31 (Springer Singapore, 2016).

31 Loëwenstein, W. The distribution of aluminum in the tetrahedra of silicates and aluminates American Mineralogist 39, 92-96 (1954).

32 Fletcher, R. E., Ling, S. \& Slater, B. Violations of Lowenstein's rule in zeolites. Chemical Science 8, 7483-7491, doi:10.1039/C7SC02531A (2017).

33 Nakano, T. \& Nozue, Y. Orbital degeneracy and magnetic properties of potassium clusters incorporated into nanoporous crystals of zeolite A. Journal of Computational Methods in Sciences and Engineering 7, 443-462 (2007).

34 Framework Type Codes, www.iza-structure.org/IZA-SC_FTC_list.htm (2017).

35 Yaghi, O. M. \& Li, H. Hydrothermal Synthesis of a Metal-Organic Framework Containing Large Rectangular Channels. Journal of the American Chemical Society 117, 10401-10402, doi:10.1021/ja00146a033 (1995). 
36 Furukawa, H., Cordova, K. E., O’Keeffe, M. \& Yaghi, O. M. The Chemistry and Applications of Metal-Organic Frameworks. Science 341, doi:10.1126/science.1230444 (2013).

37 Moghadam, P. Z., Li, A., Wiggin, S. B., Tao, A., Maloney, A. G. P., Wood, P. A., Ward, S. C. \& Fairen-Jimenez, D. Development of a Cambridge Structural Database Subset: A Collection of Metal-Organic Frameworks for Past, Present, and Future. Chemistry of Materials 29, 2618-2625, doi:10.1021/acs.chemmater.7b00441 (2017).

Allen, F. The Cambridge Structural Database: a quarter of a million crystal structures and rising. Acta Crystallographica Section B 58, 380-388, doi:10.1107/S0108768102003890 (2002).

39 Batten, S. R., Champness, N. R., Chen, X. M., Garcia-Martinez, J., Kitagawa, S., Ohrstrom, L., O'Keeffe, M., Suh, M. P. \& Reedijk, J. Terminology of metal-organic frameworks and coordination polymers (IUPAC Recommendations 2013). Pure and Applied Chemistry 85, 1715-1724, doi:10.1351/pac-rec-12-11-20 (2013).

40 Li, H., Eddaoudi, M., O'Keeffe, M. \& Yaghi, O. M. Design and synthesis of an exceptionally stable and highly porous metal-organic framework. Nature 402, 276-279, doi:10.1038/46248 (1999).

41 Chui, S. S.-Y., Lo, S. M.-F., Charmant, J. P. H., Orpen, A. G. \& Williams, I. D. A Chemically Functionalizable Nanoporous Material $\left[\mathrm{Cu}_{3}(\mathrm{TMA})_{2}\left(\mathrm{H}_{2} \mathrm{O}\right)_{3}\right]_{\mathrm{n}}$. Science 283, 1148-1150, doi:10.1126/science.283.5405.1148 (1999).

42 Yaghi, O. M., O'Keeffe, M., Ockwig, N. W., Chae, H. K., Eddaoudi, M. \& Kim, J. Reticular synthesis and the design of new materials. Nature 423, 705-714, doi:10.1038/nature01650 (2003).

43 Eddaoudi, M., Kim, J., Rosi, N., Vodak, D., Wachter, J., O'Keeffe, M. \& Yaghi, O. M. Systematic Design of Pore Size and Functionality in Isoreticular MOFs and Their Application in Methane Storage. Science 295, 469-472, doi:10.1126/science.1067208 (2002).

44 Horike, S., Shimomura, S. \& Kitagawa, S. Soft porous crystals. Nature Chemistry, 1, 695-704, doi:10.1038/nchem.444 (2009). 
45 Coudert, F. X. Responsive Metal-Organic Frameworks and Framework Materials: Under Pressure, Taking the Heat, in the Spotlight, with Friends. Chemistry of Materials 27, 1905-1916, doi:10.1021/acs.chemmater.5b00046 (2015).

46 Tian, T., Zeng, Z., Vulpe, D., Casco, M. E., Divitini, G., Midgley, P. A., Silvestre-Albero, J., Tan, J.-C., Moghadam, P. Z. \& Fairen-Jimenez, D. A solgel monolithic metal-organic framework with enhanced methane uptake. Nature Materials, doi:10.1038/nmat5050 (2017).

47 Peplow, M. Materials science: The hole story. Nature 520, 148-150, doi:10.1038/520148a (2015).

48 Frameworks for commercial success. Nature Chemistry, 8, 987, doi:10.1038/nchem.2661 (2016).

49 Perez, E. V., Balkus, K. J., Ferraris, J. P. \& Musselman, I. H. Metal-organic polyhedra 18 mixed-matrix membranes for gas separation. Journal of Membrane Science 463, 82-93, doi:https://doi.org/10.1016/j.memsci.2014 $03.045(2014)$.

50 Jones, J. T. A., Holden, D., Mitra, T., Hasell, T., Adams, D. J., Jelfs, K. E., Trewin, A., Willock, D. J., Day, G. M., Bacsa, J., Steiner, A. \& Cooper, A. I. On-Off Porosity Switching in a Molecular Organic Solid. Angewandte Chemie International Edition 50, 749-753, doi:10.1002/anie.201006030 (2011).

51 Mastalerz, M. \& Oppel, I. M. Rational Construction of an Extrinsic Porous Molecular Crystal with an Extraordinary High Specific Surface Area. Angewandte Chemie International Edition 51, 5252-5255, doi:10.1002/anie.201201174 (2012).

52 Cooper, A. I. Porous Molecular Solids and Liquids. ACS Central Science 3, 544-553, doi:10.1021/acscentsci.7b00146 (2017).

53 Tozawa, T., Jones, J. T. A., Swamy, S. I., Jiang, S., Adams, D. J., Shakespeare, S., Clowes, R., Bradshaw, D., Hasell, T., Chong, S. Y., Tang, C., Thompson, S., Parker, J., Trewin, A., Bacsa, J., Slawin, A. M. Z., Steiner, A. \& Cooper, A. I. Porous organic cages. Nature Materials 8, 973-978, doi:10.1038/nmat2545 (2009). 
54 Barbour, L. J. Crystal porosity and the burden of proof. Chemical Communications, 1163-1168, doi:10.1039/B515612M (2006).

55 Evans, J. D., Fraux, G., Gaillac, R., Kohen, D., Trousselet, F., Vanson, J.-M. \& Coudert, F.-X. Computational Chemistry Methods for Nanoporous Materials. Chemistry of Materials 29, 199-212, doi:10.1021/acs.chemmater.6b02994 (2017).

56 Evans, J. D., Huang, D. M., Haranczyk, M., Thornton, A. W., Sumby, C. J. \& Doonan, C. J. Computational identification of organic porous molecular crystals. Crystengcomm 18, 4133-4141, doi:10.1039/C6CE00064A (2016). computational methods to the design and characterisation of porous molecular materials. Chemical Society Reviews 46, 3286-3301, doi:10.1039/C7CS00084G (2017).

58 Pulido, A., Chen, L., Kaczorowski, T., Holden, D., Little, M. A., Chong, S. Y., Slater, B. J., McMahon, D. P., Bonillo, B., Stackhouse, C. J., Stephenson, A., Kane, C. M., Clowes, R., Hasell, T., Cooper, A. I. \& Day, G. M. Functional materials discovery using energy-structure-function maps. Nature 543, 657-664, doi:10.1038/nature21419 (2017).

59 Slater, A. G., Reiss, P. S., Pulido, A., Little, M. A., Holden, D. L., Chen, L., Chong, S. Y., Alston, B. M., Clowes, R., Haranczyk, M., Briggs, M. E., Hasell, T., Day, G. M. \& Cooper, A. I. Computationally-Guided Synthetic Control over Pore Size in Isostructural Porous Organic Cages. ACS Central Science 3, 734-742, doi:10.1021/acscentsci.7b00145 (2017).

60 Tan, J. C. \& Cheetham, A. K. Mechanical properties of hybrid inorganicorganic framework materials: establishing fundamental structure-property relationships. Chemical Society Reviews 40, 1059-1080, doi:10.1039/COCS00163E (2011).

61 Funnell, N. P., Marshall, W. G. \& Parsons, S. Alanine at 13.6 GPa and its pressure-induced amorphisation at 15 GPa. Crystengcomm 13, 5841-5848, doi:10.1039/C1CE05487B (2011).

62 Slebodnick, C., Zhao, J., Angel, R., Hanson, B. E., Song, Y., Liu, Z. \& Hemley, R. J. High Pressure Study of Ru3(CO)12 by X-ray Diffraction, Raman, and 
Infrared Spectroscopy. Inorganic Chemistry 43, 5245-5252, doi:10.1021/ic049617y (2004).

63 Angel, R. J. in High-pressure crystallography: status artis and emerging opportunities (eds Francesca P. A. Fabbiani, John B. Parise, \& Malcolm Guthrie) 39-50 (NATO, 2016).

64 Birch, F. Finite Elastic Strain of Cubic Crystals. Physical Review 71, 809-824 (1947).

65 Angel, R. J., Gonzalez-Platas, J. \& Alvaro, M. EosFit7c and a Fortran module (library) for equation of state calculations. Zeitschrift Fur KristallographieCrystalline Materials 229, 405-419, doi:10.1515/zkri-2013-1711 (2014).

66 Carrington, E. J., Vitorica-Yrezabal, I. J. \& Brammer, L. Crystallographic studies of gas sorption in metal-organic frameworks. Acta Crystallographica Section B 70, 404-422, doi:10.1107/S2052520614009834 (2014).

67 McKellar, S. C. \& Moggach, S. A. Structural studies of metal-organic frameworks under high pressure. Acta Crystallographica Section B 71, 587607, doi:10.1107/S2052520615018168 (2015).

68 Gatta, G. D. \& Lee, Y. Zeolites at high pressure: A review. Mineralogical Magazine 78, 267, doi:10.1180/minmag.2014.078.2.04 (2014).

69 Peterson, V. K., Liu, Y., Brown, C. M. \& Kepert, C. J. Neutron Powder Diffraction Study of $\mathrm{D}_{2}$ Sorption in $\mathrm{Cu}_{3}(1,3,5 \text {-benzenetricarboxylate })_{2}$. Journal of the American Chemical Society 128, 15578-15579, doi:10.1021/ja0660857 (2006).

70 Peterson, V. K., Brown, C. M., Liu, Y. \& Kepert, C. J. Structural Study of $\mathrm{D}_{2}$ within the Trimodal Pore System of a Metal Organic Framework. The Journal of Physical Chemistry C 115, 8851-8857, doi:10.1021/jp2010937 (2011).

71 Getzschmann, J., Senkovska, I., Wallacher, D., Tovar, M., Fairen-Jimenez, D., Düren, T., van Baten, J. M., Krishna, R. \& Kaskel, S. Methane storage mechanism in the metal-organic framework $\mathrm{Cu}_{3}(\mathrm{btc})_{2}$ : An in situ neutron diffraction study. Microporous and Mesoporous Materials 136, 50-58, doi:https://doi.org/10.1016/j.micromeso.2010.07.020 (2010). 
72 Wu, H., Simmons, J. M., Liu, Y., Brown, C. M., Wang, X.-S., Ma, S., Peterson, V. K., Southon, P. D., Kepert, C. J., Zhou, H.-C., Yildirim, T. \& Zhou, W. Metal-Organic Frameworks with Exceptionally High Methane Uptake: Where and How is Methane Stored? Chemistry - A European Journal 16, 5205-5214, doi:10.1002/chem.200902719 (2010).

73 Wu, H., Simmons, J. M., Srinivas, G., Zhou, W. \& Yildirim, T. Adsorption Sites and Binding Nature of $\mathrm{CO}_{2}$ in Prototypical Metal-Organic Frameworks: A Combined Neutron Diffraction and First-Principles Study. The Journal of Physical Chemistry Letters 1, 1946-1951, doi:10.1021/jz100558r (2010).

74 Hulvey, Z., Lawler, K. V., Qiao, Z., Zhou, J., Fairen-Jimenez, D., Snurr, R. Q., Ushakov, S. V., Navrotsky, A., Brown, C. M. \& Forster, P. M. Noble Gas Adsorption in Copper Trimesate, HKUST-1: An Experimental and Computational Study. The Journal of Physical Chemistry C 117, 20116-20126, doi:10.1021/jp408034u (2013).

75 Chapman, K. W., Halder, G. J. \& Chupas, P. J. Guest-Dependent High Pressure Phenomena in a Nanoporous Metal-Organic Framework Material. Journal of the American Chemical Society 130, 10524-10526, doi:10.1021/ja804079z (2008).

76 Graham, A. J., Tan, J.-C., Allan, D. R. \& Moggach, S. A. The effect of pressure on Cu-btc: framework compression vs. guest inclusion. Chemical Communications 48, 1535-1537, doi:10.1039/C1CC16045A (2012).

77 Zhang, C., Gee, J. A., Sholl, D. S. \& Lively, R. P. Crystal-Size-Dependent Structural Transitions in Nanoporous Crystals: Adsorption-Induced Transitions in ZIF-8. The Journal of Physical Chemistry C 118, 20727-20733, doi:10.1021/jp5081466 (2014).

78 Mohideen, M. I. H., Xiao, B., Wheatley, P. S., McKinlay, A. C., Li, Y., Slawin, A. M. Z., Aldous, D. W., Cessford, N. F., Düren, T., Zhao, X., Gill, R., Thomas, K. M., Griffin, J. M., Ashbrook, S. E. \& Morris, R. E. Protecting group and switchable pore-discriminating adsorption properties of a hydrophilic-hydrophobic metal-organic framework. Nature Chemistry, 3, 304-310, doi:10.1038/nchem.1003 (2011). 
79 McKellar, S. C., Graham, A. J., Allan, D. R., Mohideen, M. I. H., Morris, R. E. \& Moggach, S. A. The effect of pressure on the post-synthetic modification of a nanoporous metal-organic framework. Nanoscale 6, 41634173, doi:10.1039/c3nr04161a (2014).

80 Wang, Z. \& Cohen, S. M. Postsynthetic Covalent Modification of a Neutral Metal-Organic Framework. Journal of the American Chemical Society 129, 12368-12369, doi:10.1021/ja074366o (2007).

81 Cohen, S. M. Modifying MOFs: new chemistry, new materials. Chemical Science 1, 32-36, doi:10.1039/COSC00127A (2010).

82 Tanabe, K. K. \& Cohen, S. M. Postsynthetic modification of metal-organic frameworks-a progress report. Chemical Society Reviews 40, 498-519, doi:10.1039/C0CS00031K (2011).

83 Wang, Z. \& Cohen, S. M. Postsynthetic modification of metal-organic frameworks. Chemical Society Reviews 38, 1315-1329, doi:10.1039/B802258P (2009).

84 Wu, H., Zhou, W. \& Yildirim, T. Methane Sorption in Nanoporous Metal-Organic Frameworks and First-Order Phase Transition of Confined Methane. The Journal of Physical Chemistry C 113, 3029-3035, doi:10.1021/jp8103276 (2009).

85 Wu, H., Zhou, W. \& Yildirim, T. Hydrogen Storage in a Prototypical Zeolitic Imidazolate Framework-8. Journal of the American Chemical Society 129, 5314-5315, doi:10.1021/ja0691932 (2007).

86 Fairen-Jimenez, D., Moggach, S. A., Wharmby, M. T., Wright, P. A., Parsons, S. \& Düren, T. Opening the Gate: Framework Flexibility in ZIF-8 Explored by Experiments and Simulations. Journal of the American Chemical Society 133, 8900-8902, doi:10.1021/ja202154j (2011).

87 Zhou, W., Wu, H., Hartman, M. R. \& Yildirim, T. Hydrogen and Methane Adsorption in Metal-Organic Frameworks: A High-Pressure Volumetric Study. The Journal of Physical Chemistry C 111, 16131-16137, doi:10.1021/jp074889i (2007).

88 Moggach, S. A., Bennett, T. D. \& Cheetham, A. K. The Effect of Pressure on ZIF-8: Increasing Pore Size with Pressure and the Formation of a High- 
Pressure Phase at 1.47 GPa. Angewandte Chemie International Edition 48, 7087-7089, doi:10.1002/anie.200902643 (2009).

89 Miller, S. R., Wright, P. A., Serre, C., Loiseau, T., Marrot, J. \& Ferey, G. A microporous scandium terephthalate, $\mathrm{Sc}_{2}\left(\mathrm{O}_{2} \mathrm{CC}_{6} \mathrm{H}_{4} \mathrm{CO}_{2}\right)_{3}$, with high thermal stability. Chemical Communications, 3850-3852, doi:10.1039/B506677H (2005).

90 Mowat, J. P. S., Miller, S. R., Griffin, J. M., Seymour, V. R., Ashbrook, S. E., Thompson, S. P., Fairen-Jimenez, D., Banu, A.-M., Düren, T. \& Wright, P. A. Structural Chemistry, Monoclinic-to-Orthorhombic Phase Transition, and $\mathrm{CO}_{2}$ Adsorption Behavior of the Small Pore Scandium Terephthalate, $\mathrm{Sc}_{2}\left(\mathrm{O}_{2} \mathrm{CC}_{6} \mathrm{H}_{4} \mathrm{CO}_{2}\right)_{3}$, and Its Nitro- And Amino-Functionalized Derivatives. Inorganic Chemistry 50, 10844-10858, doi:10.1021/ic201387d (2011).

91 Miller, S. R., Wright, P. A., Devic, T., Serre, C., Férey, G., Llewellyn, P. L., Denoyel, R., Gaberova, L. \& Filinchuk, Y. Single Crystal X-ray Diffraction Studies of Carbon Dioxide and Fuel-Related Gases Adsorbed on the Small Pore Scandium Terephthalate Metal Organic Framework, $\mathrm{Sc}_{2}\left(\mathrm{O}_{2} \mathrm{CC}_{6} \mathrm{H}_{4} \mathrm{CO}_{2}\right)_{3}$. Langmuir 25, 3618-3626, doi:10.1021/la803788u (2009).

92 Graham, A. J., Banu, A.-M., Dueren, T., Greenaway, A., McKellar, S. C., Mowat, J. P. S., Ward, K., Wright, P. A. \& Moggach, S. A. Stabilization of Scandium Terephthalate MOFs against Reversible Amorphization and Structural Phase Transition by Guest Uptake at Extreme Pressure. Journal of the American Chemical Society 136, 8606-8613, doi:10.1021/ja411934f (2014). 
- Chapter 2 Crystallographic Methods 


\subsection{Beamline I19}

I19 at the Diamond Light Source is set-up as a dedicated small molecule single crystal X-ray diffraction beamline, utilising an in-vacuum U21 undulator as the source of synchrotron radiation. ${ }^{1}$ The beamline consists of an optics hutch and two experimental hutches, EH1 and EH2. Following a recent upgrade, EH1 is now dedicated to high throughput structural chemistry studies allowing remote access, automatic data collection and processing more akin to the most advanced macromolecular beamlines. EH2 provides a platform and space for a variety of different sample environment set-ups to map structural changes under the influence of external stimuli such as; temperature (open-flow and closed-cycle cryostats or open-flow furnace), light (for photo-excited state studies), pressure (using diamond anvil cells), and exposure to gases (using quartz or sapphire capillaries on individual gases or gas mixtures). Samples can also be studied using a combination of these techniques.

EH2 is equipped with a Newport four-circle kappa geometry goniometer which can support loads of up to $25 \mathrm{~kg}$ on the sample position and, at the time of manufacture, the sphere of confusion was measured to be $<60 \mu \mathrm{m}$. The instrument is fitted with a PILATUS $300 \mathrm{~K}$ photon counting pixel array detector that allows rapid shutterless collections. The photon energy can be tuned in the range $5 \mathrm{keV}$ to $25 \mathrm{keV}$ (corresponding to a wavelength range of $2.5 \AA$ to $0.5 \AA$ ).

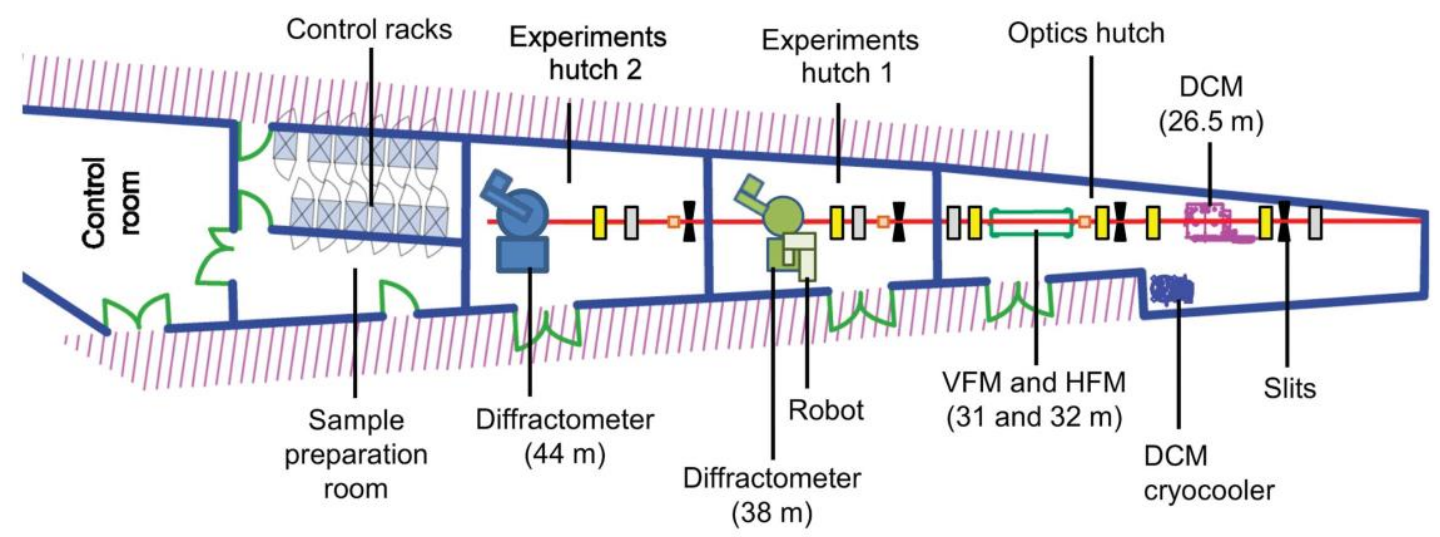

Figure 2.1 - Layout of the I19 beamline, showing the two experimental hutches in which single-crystal diffraction experiments are carried out. Picture from I19 website. 
A large number of the experiments presented in this thesis were conducted on I19. Provided here is a general overview of the practical aspects of the high-pressure techniques that are used in Chapters $5 \& 6$. This is followed by a brief review of gas cells and a technical overview of the gas cell set-up in EH2 used in Chapter 7. 


\subsection{High-pressure crystallographic methods}

For many years generating extremely high pressures posed a significant technical challenge, ${ }^{2}$ however, with the development of the diamond anvil cell (DAC), and subsequent technical innovations, generating pressures in excess of 1 GPa (10 kbar) is now routine. ${ }^{3}$ Pressure is defined as the amount of force applied per unit area. ${ }^{4}$ The maximum force that can be applied is limited by the ultimate strength of the materials used and so to generate very high pressures, the area over which that force is applied must be minimised.

The DAC was first developed in 1958 (Figure 2.2a), and uses the exceptional strength of diamond under compression and the shape of the opposing anvils to reduce the area to generate extreme pressures. ${ }^{22}$ Early designs worked around a hinge and lever mechanism, but this fell out of favour to be replaced by the pin aligned vertical displacement cell..$^{5}$ The adaptability of size and shape of DACs have allowed them to be used for many different experimental setups, including spectroscopic measurements (Raman, IR, UV/vis) ${ }^{6}$, magnetic measurements (SQUID, EPR), ${ }^{7}$ and diffraction (X-ray \& neutron: powder \& single crystal). ${ }^{8}$ The history and early development of the DAC is well documented in retrospective pieces from W. Basset and G. Piermarini. 9,10
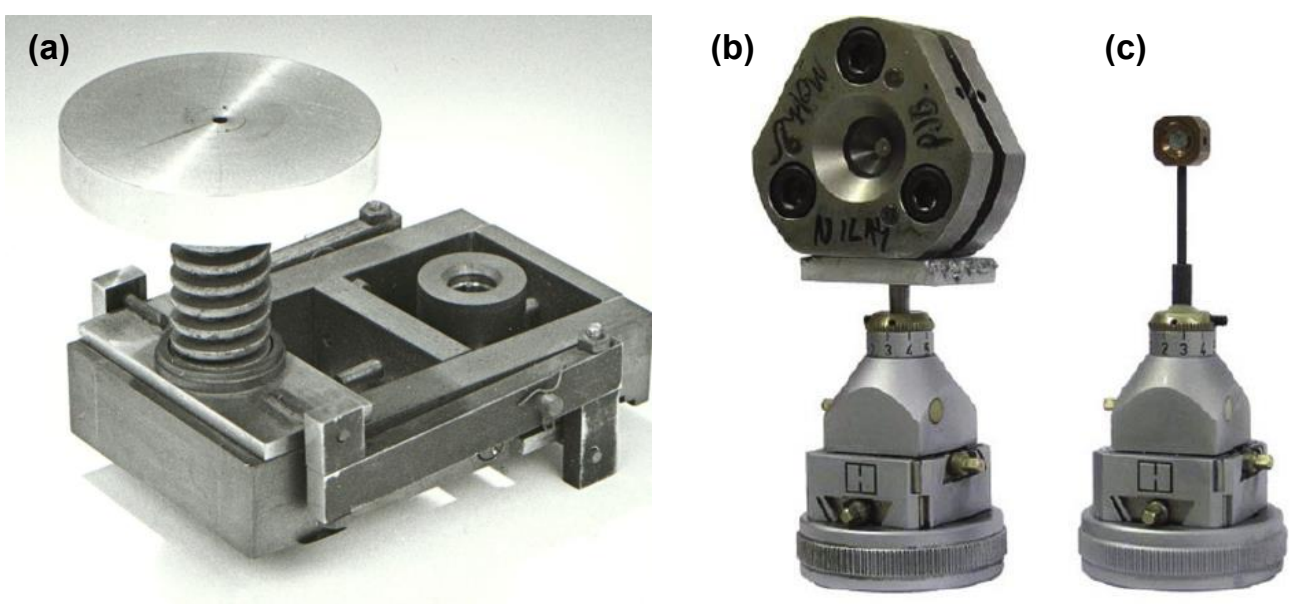

Figure 2.2 - (a) The original diamond anvil pressure cell handmade by C. E. Weir at the National Bureau of Standards (NBS) in 1957-58, image taken from Piermarini, 2001. A Merrill \& Bassett DAC (b) alongside a Mini-DAC turn-buckle style cell (c) mounted onto goniometer heads. Image taken from Ridley \& Kamenev, 2014. 
High-pressure single-crystal X-ray diffraction measurements were first taken in 1965 using a custom built leaver arm DAC and heavily modified precession camera. ${ }^{5,6}$ Fortunately, since this time, the process has become somewhat simpler. Innovations such as the Merrill \& Bassett DAC, Figure 2.2b, made the field far more accessible as they allowed high-pressure experiments to be performed using standard lab diffractometers for relatively little initial expense. ${ }^{11}$ The Merrill \& Bassett DAC has been the basis for many further improvements and variations making it one of the most common DAC's used today. Here a modified MerrilBassett DAC, Figure 2.3, will be described in detail as this is the cell that was used for experiments presented here in Chapters $5 \& 6 .^{12}$

The DAC generates its pressure by using two Boehler-Almax conical cut diamonds that have a flat face (a culet) ground onto the surface of the diamond with a diameter of $600 \mu \mathrm{m} .{ }^{13}$ These two diamonds are aligned one on top of the other to make the two culets parallel and concentric. A metal gasket with a small hole concentric to the culets of 200-300 $\mu \mathrm{m}$ in diameter is placed between the diamonds. ${ }^{14}$ Gasket materials are commonly made from ductile metals such as steel, beryllium or tungsten. The gasket hole and the thickness of the gasket create a small cylindrical experimental chamber that contains the sample. The diamonds are supported by tungsten carbide backing disks which are in turn held by the

(a)

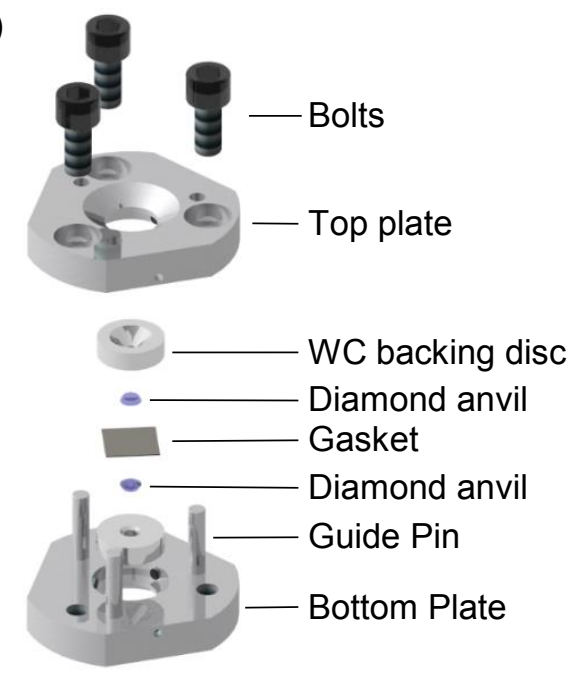

(b)

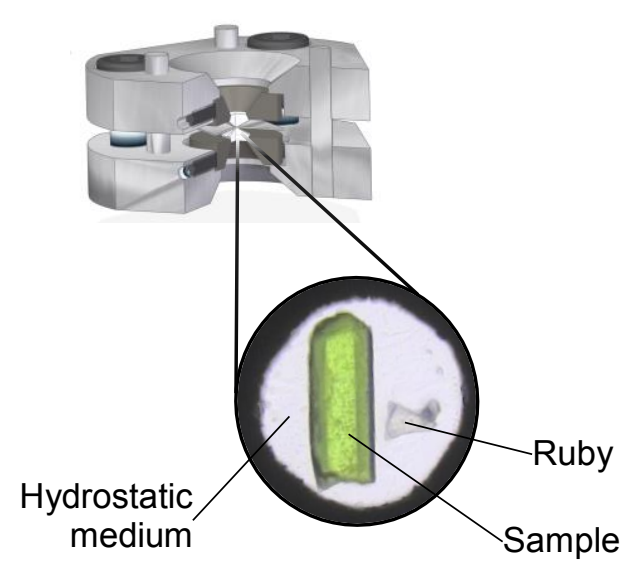

Figure 2.3 - (a) An exploded model of a modified Merrill \& Bassett DAC showing its different components. (b) A sectioned model of an assembled DAC. Insert of the view looking through the diamonds to reveal the sample chamber. 
steel body of the cell. The whole assembly is clamped together axially using guide pins for alignment and the Allen screws for applying pressure. The cell body and backing disks are chamfered to expose the back of the diamond to allow the sample to be viewed whilst under pressure and provide an opening angle $\left(80^{\circ}\right)$ for the incoming and diffracted X-rays.

As the two opposing sides of the cell come together, the diamonds seal against the gasket, at which point the pressure in the sample chamber increases dramatically. To measure the pressure inside the sample chamber during the experiment a small chip of ruby is also placed in the sample chamber, as it has a characteristic fluorescence transition which is dependent on pressure. Ruby is commonly used as the shift in fluorescence is well defined up to $20 \mathrm{GPa}$, and simple to measure, however other pressure calibrants can be used. ${ }^{15-17}$

To ensure that the pressure is applied evenly to the sample a pressure transmitting medium (PTM) is used to fill the remaining sample chamber volume and can be a liquid, gas, or even a soft solid. The choice of PTM can make a large difference to the experiment. Typically gasses ( $\mathrm{He}, \mathrm{Ar}, \mathrm{N}_{2}$ etc.) provide the highest hydrostatic limits, which equates to the point where the pressure across the experimental volume becomes inhomogeneous and differential. ${ }^{18}$ Liquid PTMs are common as they are simple to load into the DAC, however they generally have much more modest hydrostatic limits, Table 2.1.

Table 2.1 - Hydrostatic limits of various PTMs taken from S. Klotz et.al. ${ }^{18}$

\begin{tabular}{ccc}
\hline \multicolumn{2}{c}{ Pressure transmitting medium } & Hydrostatic limit \\
\hline Iso-n-pentane & $1: 1$ iso-n pentane & $7.4 \mathrm{GPa}$ \\
mixtures & $5: 1$ iso-n pentane & $7.4 \mathrm{GPa}$ \\
Methanol- & $4: 1$ methanol-ethanol & $\sim 10.5 \mathrm{GPa}$ \\
ethanol mixtures & $16: 3: 1$ methanol-ethanol-water & $\sim 10.5 \mathrm{GPa}$ \\
Fluorinert & $1: 1$ FC84-FC87 Fluorinert & $2.3 \mathrm{GPa}$ \\
Oils & Daphne 7474 & $3.7 \mathrm{GPa}$ \\
& Silicone oil & $3 \mathrm{GPa}$ \\
Gasses & Nitrogen & $\sim 13 \mathrm{GPa}$ \\
& Neon & $\sim 15 \mathrm{GPa}$ \\
& Argon & $\sim 10 \mathrm{GPa}$ \\
& Helium & $\sim 25 \mathrm{GPa}$ \\
\hline
\end{tabular}


PTMs are usually chosen as they are inert to the sample, and cause direct compression, but there is also the option to use a medium that can interact or react with the sample. This is particularly relevant to porous materials as the PTM can penetrate the pores. The size of the molecules encompassing the PTM therefore determines whether the fluid will directly compress the material, will be non-penetrating, or enter the pores and interact as a penetrating medium. Examples of this were shown in the introduction, and this effect will be central to discussions presented throughout this thesis.

The high-pressure diffraction experiments described here were either carried out using a Bruker-Nonius APEX-II diffractometer with a CCD detector, Figure 2.4a, or on beamline I19, EHl, using a Crystal Logic four-circle goniometer equipped with a Rigaku Saturn 724+ CCD detector. This diffractometer has since been replaced with a recent upgrade to EHl. More details can be found in the appropriate experimental sections of each chapter.

The data collection strategy for high-pressure experiments has to take into consideration the large areas of reciprocal space that are shaded by the cell body. Therefore diffraction data can only be acquired at limited values of $\omega$ and $\phi$. Based on the work by Dawson et al. for a DAC with an opening angle of $80^{\circ}$, a strategy
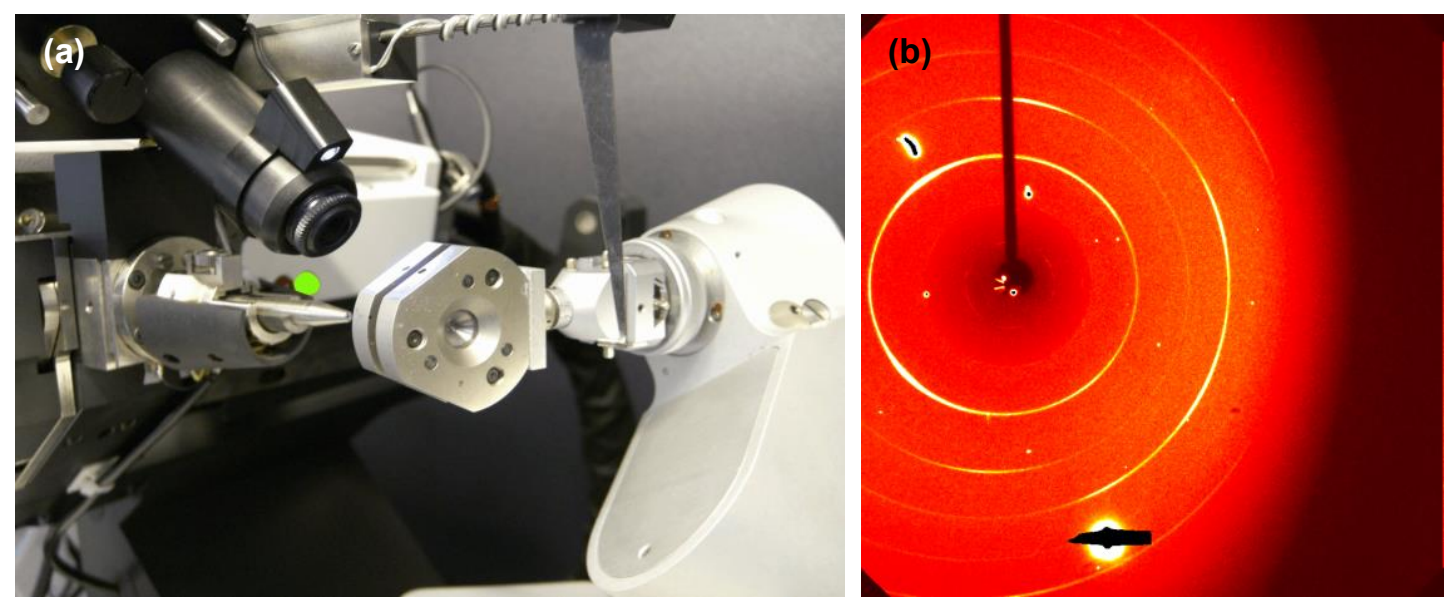

Figure 2.4 - (a) The DAC in place on the diffractometer. (b) A typical frame from a high-pressure experiment. Note the overloaded reflections from the diamond anvils, the Debye-Scherrer rings from the gasket, and the shaded region from the cell body. 
that ensures that the angle between the incident beam and the cell axis is less than $40^{\circ}$ and that a maximum of $80 \%$ of the detector is shaded works best. ${ }^{19}$ A typical strategy therefore consists of a series of $\omega$ scans at eight settings of $2 \theta$ and $\phi$. To improve data completeness different settings of $\chi$ can also be utilised. On fixed $\chi$ instruments this can be simulated by re-collecting data with the pressure cell attached to the goniometer head on each of its three sides and the data merged after processing.

Table 2.2 - Typical run list of a high-pressure diffraction experiment on a fixed $\chi$ diffractometer.

\begin{tabular}{cccccc}
\hline Run & $\begin{array}{c}\text { Detector } \\
\text { Distance }(\mathbf{m m})\end{array}$ & $\mathbf{X}\left({ }^{\circ}\right)$ & $\mathbf{2 \theta}\left({ }^{\circ}\right)$ & $\boldsymbol{\varphi}\left(^{\circ}\right)$ & $\boldsymbol{\omega}\left({ }^{\circ}\right)$ \\
\hline 1 & 72 & 54.7 & -28 & 90 & -10 to -40 \\
2 & 72 & 54.7 & 28 & 90 & 40 to -25 \\
3 & 72 & 54.7 & -28 & 90 & -155 to -220 \\
4 & 72 & 54.7 & 28 & 90 & -140 to -170 \\
5 & 72 & 54.7 & -28 & 270 & -155 to -220 \\
6 & 72 & 54.7 & 28 & 270 & -140 to -170 \\
7 & 72 & 54.7 & -28 & 270 & -10 to -40 \\
8 & 72 & 54.7 & 28 & 270 & 40 to -25 \\
\hline
\end{tabular}

Data reduction for high-pressure single-crystal diffraction requires multiple steps over and above routine single-crystal diffraction experiments. For unit cell indexing, the harvested reflections include peaks from not only the sample but the ruby chip and the diamond anvils. Debye-Scherrer rings from the gasket are also present and, at occluded geometry's, a shaded region from the cell body.

A reciprocal lattice viewer can be used to 'clean up' the harvested reflections to remove those from the ruby and diamond leaving only reflections from the sample. Powder diffraction rings can be removed in the same way, or these regions masked during harvesting reflections. During integration, it is important to mask regions of the frames that are shaded by the cell body, Figure 2.4. This can be done using a predetermined set of dynamic masks 20 or created on-the-fly during integration. ${ }^{21-23}$ Scaling and multi-scan absorption corrections are then applied. ${ }^{24}$ When all of these aspects are taken into account, data quality obtained from highpressure experiments can be very high and the errors on the structural parameters are often comparable to data obtained under ambient conditions. 
Despite the many different designs throughout the evolution of the DAC there are some fundamental issues that are present in all opposed anvil pressure cells. Foremost of these is the reduced completeness due to the shading by the cell body. This can be improved by increasing the opening angle, allowing a greater volume of reciprocal space to be accessed, however there is a limit to how open this angle can be while retaining the necessary mechanical strength to achieve high pressures. Reducing the wavelength of the radiation allows more reciprocal space to be accessed, but this comes with a trade-off of decreased diffracted intensities. This is usually overcome by using synchrotron sources which can produce very brilliance X-rays at short wavelengths. However, low symmetry crystals will usually always suffer from reduced completeness that can make structures difficult to study.

The DAC was designed principally as a high-pressure device (in excess of 2000 bar, 2 kbar or $0.2 \mathrm{GPa}$ ) and it excels at this, comfortably spanning 6 orders of magnitude of pressure. However, upon closing the cell, the diamonds have to form a seal with the gasket, and this requires a certain level of load. Therefore, the lowest attainable pressures are typically in the region of $0.3-0.4 \mathrm{GPa}(3-4 \mathrm{kbar})$, and for a typical mechanical DAC increasing the pressure by less than 0.1 GPa (1 kbar) at a time is virtually impossible. As a consequence, 3 - 5 kbar steps are commonly applied. Many different designs of DACs have been employed to reduce the pressure step size and sealing pressure such as the use of a gas membrane to apply the load on the cell and very large cutlets $(1.4 \mathrm{~mm}) .{ }^{25-28}$ Despite these efforts the minimum accurately attainable pressures is still in the order of 1,000's of bar.

Simple measurement of the pressure inside the DAC is possible through ruby fluorescence. ${ }^{15}$ This is by far the most common method employed but the hardness of ruby means that the shifts in fluorescence are only very slight until significant changes in pressure are achieved. A $0.01 \mathrm{~nm}$ shift, for example, corresponds to a $\sim 274$ bar shift in pressure. The fluorescence is also highly temperature sensitive, 5-6 K temperature changes for example equate to the same shift as a $1 \mathrm{kbar}$ pressure change. ${ }^{29}$ Therefore, even if small increments in pressure were possible mechanically, it is difficult to measure accurately using this method. More 
sensitive fluorescent pressure calibrants are available such as FluoSpheres ${ }^{\circledR}$ but there use is limited due to stability and measurement difficulties. ${ }^{17}$ Diffraction pressure calibrants can be used, and standards such as quartz, ${ }^{16}$ sodium chloride $(\mathrm{NaCl}),{ }^{30,31}$ and calcium fluoride $\left(\mathrm{CaF}_{2}\right)^{32,33}$ have well-defined equations of state in their respective pressure regions allowing the pressure to be calculated from their unit cell parameters. These can provide a more sensitive measure of the pressure than ruby but the drawback in using diffraction calibrants is that the pressure can only be determined after the diffraction measurement has been made. 


\subsection{Gas pressure cells}

Note - The gas cell set-up on I19 is constantly evolving and adapting to the needs of users to provide a state-of-the-art facility, therefore, the description here describes the set-up at the time of writing (2017).

Gas pressure cells for diffraction experiments have seen a recent resurgence of interest, particularly with the rise in interest in porous materials. Since 2008, at least ten new cells have been developed and are mainly found at central facilities. ${ }^{34-44}$ Gas pressure cells broadly fall into two categories: environmental modification cells and pressure cells.

Environmental modification cells aim to replace the atmosphere in the cell with another gas, but not generate extremely high-pressures, with a maximum operating pressure of 1.5 bar. The simplest solution to this is offered by backfilling evacuated capillaries containing the sample with the desired gas, and then flamesealing the top to create a gas cell (up to 1 bar). ${ }^{42,45}$ This method fixes the pressure inside the cell and requires a new cell and crystal for each pressure.

A more sophisticated design uses a thin-walled closed-ended capillary $(0.01 \mathrm{~mm}$ wall thickness) as a sample chamber. This can be made from borosilicate glass 43 or fused quartz ${ }^{35,45}$ with a large internal diameter (ID) ranging from $1.5-3.5 \mathrm{~mm}$. Inside the capillary the crystal is mounted onto a fibre and there is an inlet for the gas. In the design by Cox et al. the inlet consisted of a bent hypodermic needle, which was connected via hoses to an offline gas source. In some cases, a separate vacuum setup which allowed the pressure and composition of the atmosphere to be altered was also added.

Pressure cells are designed to withstand gas pressures up to 300 bar. These higher pressures present an increased technical challenge which include building a capillary capable of withstanding these pressures, and a means to deliver the gas to the cell. To overcome these challenges, capillaries with larger wall thicknesses (which can be closed or open-ended) are used along with high-pressure metal capillary tubing. These two factors make pressure cells harder to implement for single-crystal diffraction measurements using monochromatic radiation, as at 
least three circles on the diffractometer are required to move in-order to collect complete data to high resolution.

Chupas et al. developed (at the Advanced Photon Source (APS), USA) an openended capillary supported in a frame that allowed gasses to flow over crystalline powdered samples at pressures up to 140 bar. ${ }^{44}$ Heating elements were also placed close to the capillary to allow heating up to $973 \mathrm{~K}$. Jensen et al. (at MAX-II, MAX$\mathrm{LAB}$, Sweden) extended the pressure and temperature range of this cell to $300 \mathrm{bar}$ and $1273 \mathrm{~K}$. A lower pressure version rated to 100 bar using a quartz sealed capillary has also been used extensively. ${ }^{41}$ Andrieux et al. (at the European Synchrotron Radiation Facility (ESRF), France), also developed a similar designed cell rated to 200 bar and $1273 \mathrm{~K}$, but with improved gas flow. This cell also allowed gas-mixtures to be studied. ${ }^{37}$ The cells designed by Chupas et al., Jensen et al. and Andrieux et al. were developed for powder diffraction measurements using synchrotron radiation.

One of the very few pressure gas cells designed for single crystal X-ray diffraction is also one of the oldest designs from Tilton, 1988. ${ }^{46}$ The sample chamber of the Tilton cell was a beryllium capillary with an ID of $1.1 \mathrm{~mm}$ and an outside diameter (OD) of $3.2 \mathrm{~mm}$, and was rated to 300 bar. The cell was affixed to a stainless steel base that had ports for the incoming gas line. One disadvantage of this design, was that the sample was not visible, making centring challenging. This design, and its limitations are very similar to the moderate pressure cells that are reviewed later in Chapter 3. 
(a)<smiles>O=CC(Br)(Br)Br</smiles>

- Internal bolt

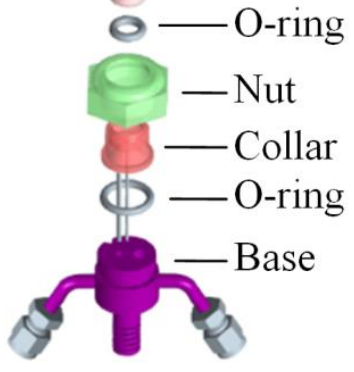

(b)

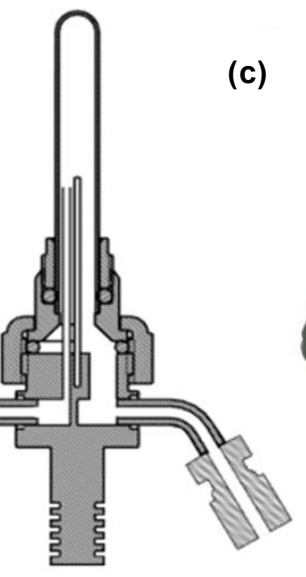

(c)

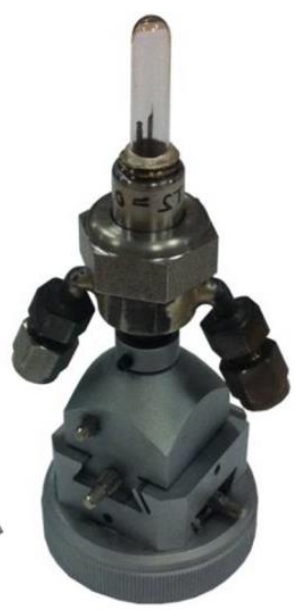

Figure 2.5 - The flow cell, (a) exploded view with parts labelled, (b) in cut away cross section and (c) mounted on to a standard XYZ goniometer head. Images courtesy of Dr Mark Warren.

The gas cell set-up developed on beamline I19 at the Diamond Light Source was used for the experiments presented in Chapter 7. There are several types of gascell available on I19, all based on a closed capillary design, and two modes are available: flow and static.

The flow cell is primarily used for environmental modification allowing the atmosphere around the sample to be exchanged constantly, Figure 2.5. This design consists of a large quartz capillary $(O D=5 \mathrm{~mm}$, length $=22 \mathrm{~mm})$. The flow cell is capable of withstanding high vacuum $\left(10^{-5} \mathrm{mbar}\right)$ to 10 bar of pressure and a maximum flow rate of $130 \mathrm{ml} / \mathrm{min}$.

The static cells are simpler than the flow cell as they do not require an inlet pipe inside the capillary to allow circulation of gas. The OD of the quartz capillary can therefore be much smaller ( $O D=1 \mathrm{~mm}$ ), allowing for higher pressures to be used, with an operational range of $10^{-5} \mathrm{mbar}$ to 100 bar. Sapphire capillaries have been used ( $1 \mathrm{~mm}$ OD and $0.6 \mathrm{~mm}$ ID) to access higher pressures still, up to 200 bar. When using static cells, it was necessary to evacuate the cell and pressure lines completely under high vacuum to ensure the gas surrounding the sample was pure. 
There are a variety of ways to attach the cells to the diffractometer. A standard $\mathrm{XYZ}$ goniometer head with IUCr standard goniometer mount can be used, as shown for the flow cell in Figure 2.5, however, as the goniometer in EH2 Is equipped with a motorised XYZ stage on $\phi$, a custom direct mount was used. This allowed sample changes to be quicker and simpler whilst also increasing the rigidity of the set-up, Figure 2.6.

In both cases the sample was mounted onto a standard MiTeGen ${ }^{\circledast}$ loop or a glass fibre using a minimum amount of an adhesive (cyanoacrylate or epoxy), to prevent movement of the crystal during data collection. As the interaction between the sample and gas is being investigated it is vital to have a clean, dry crystal attached by the minimum surface to the mount. When selecting a crystal oils such as Paratone or Fomblin were avoided in favour of picking crystal directly from a volatile solvent to ensure clean surfaces of the crystal. ${ }^{43}$

The cells were connected to a control rig through flexible 1/16 inch stainless steel capillary tubing (Swagelok SS-T1-S-014-6ME). The static cell requires one line and for the flow cells two.

A gas control rig has been designed, and used to control which gas and flow rate is required to be delivered into the cell. A wide variety of gases are available: $\mathrm{H}_{2}$, $\mathrm{N}_{2}, \mathrm{O}_{2}, \mathrm{Ne}, \mathrm{Ar}, \mathrm{Kr}, \mathrm{CO}_{2}, \mathrm{CO}, \mathrm{CH}_{4}, \mathrm{NO}, \mathrm{N}_{2} \mathrm{O}, \mathrm{H}_{2} \mathrm{~S}$, and $\mathrm{SO}_{2}$. Mixtures of any of these gases can also be used, controlled by the mass flow control (MFC) units to allow different compositions to be dialled in 'on the fly'. There are also two vacuum

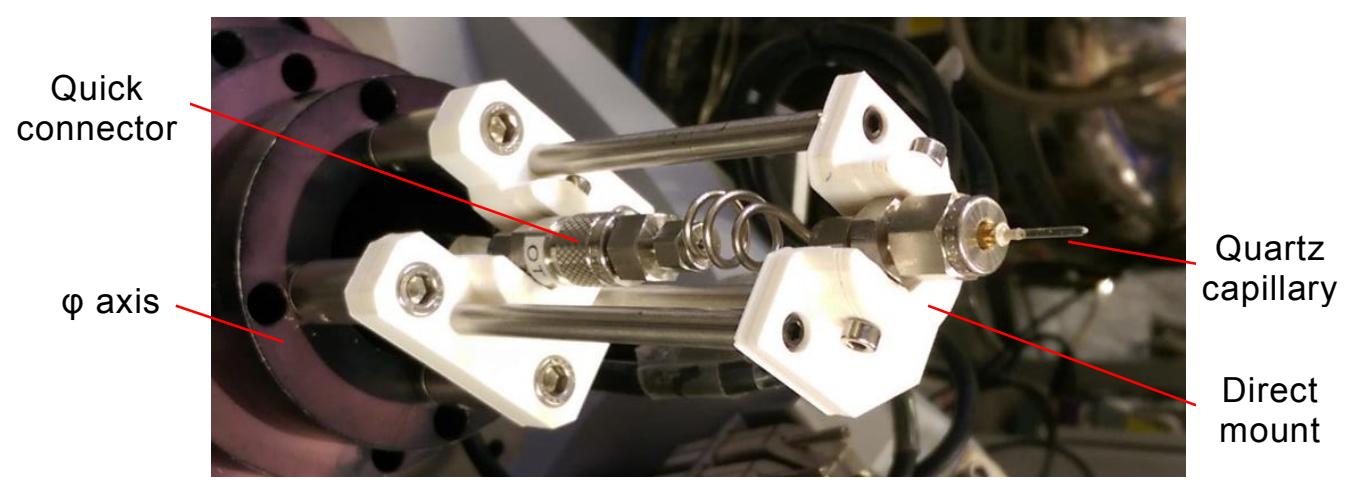

Figure 2.6 - The static quartz cell shown directly mounded to $\phi$ using a custom mount. Note the quick connector for quick sample changes. 
pumps attached to the rig, a roughing pump and an ultra-high vacuum turbo pump. The rig can be controlled remotely from outside the hutch to change pressures and temperature removing the user from the experimental hutch. This decreases the time for data collections and increases the safety of the experiment. Data collections are generally a series of $\phi$-scans (either hemispheres or full spheres depending on symmetry) to minimise tangling of the tubing coming from the cell. Processing of the frames can be carried out directly using Rigaku Oxford Diffraction software (CrysAlisPro) or CCP4 Xia2. ${ }^{21,47}$ The frames can also be converted, using ECLIPSE or CBF2SFRM, to Bruker image format and processed within the APEX 3 software suite. ${ }^{20,23,48}$

(a)
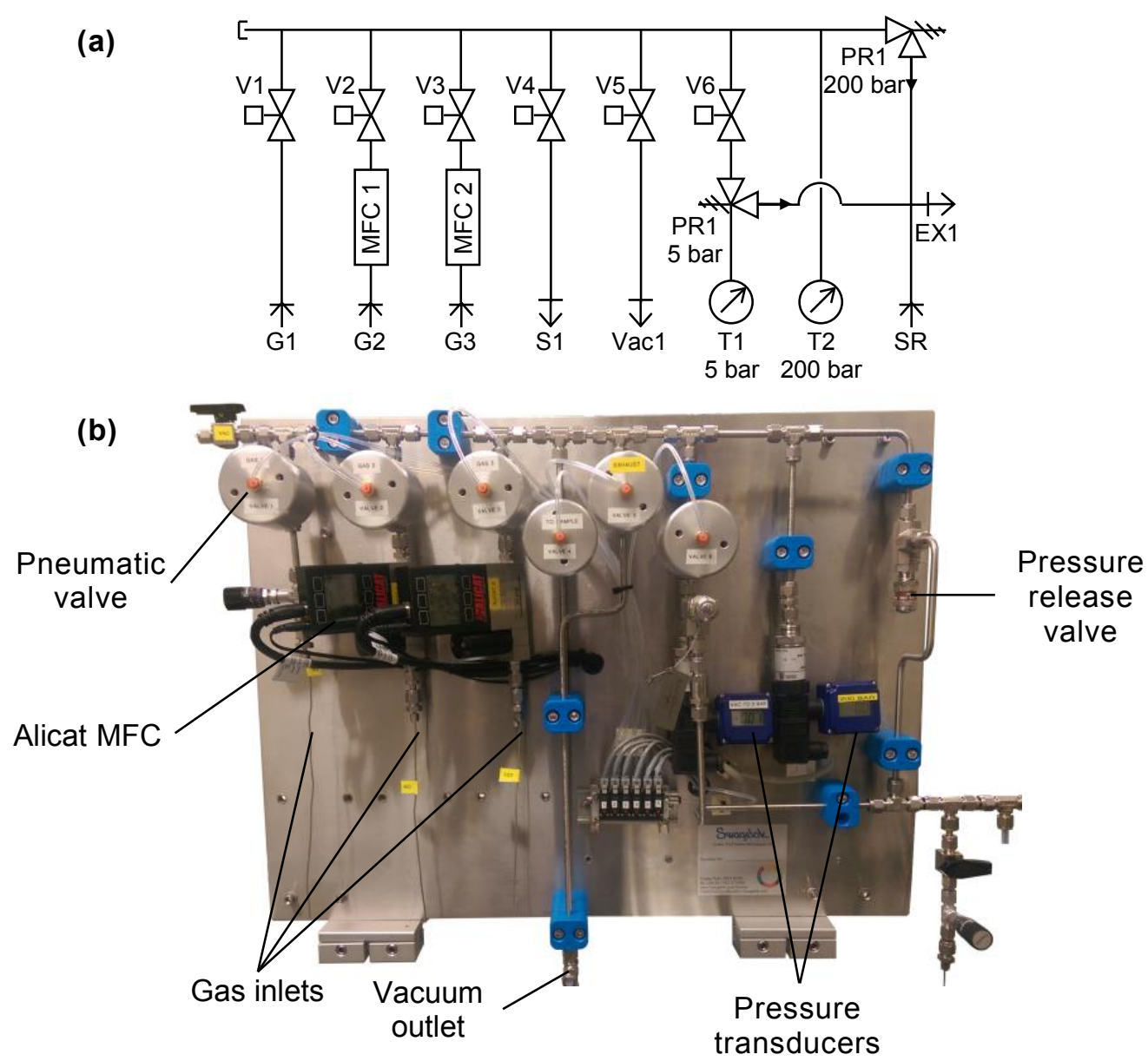

Figure 2.7 - The gas control rig available on EH2. (a) Schematic diagram of set-up and

(b) photograph of the set-up with key components labelled. Images courtesy of Dr Mark Warren. 


\subsection{Conclusions}

DACs are the clear choice for performing measurements at very high pressures, in some cases the only choice. In this role the design is extremely successful and able to cover enormous ranges of pressure. However, to investigate pressures below $0.2 \mathrm{GPa}$ (2000 bar) DACs are, for the reasons outlined above, the wrong tool for the job. Experiments at lower pressures require greater control of the pressure both in terms of the accuracy of pressure measurement and pressure step size than are possible with conventional DAC technology.

Gas cells, in particular the pressure gas cells, offer a great amount of control of the pressure but they commonly employed for in situ gas adsorption experiments rather than hydrostatic compression studies that would be comparable to typical DAC experiments. The highest useable pressure for a gas cell is normally limited by the liquefaction of the gas under pressure but the highest rated cells are in the region of 300 bar at most.

In the following chapter we present the design and use of a new pressure cell that allows access to hydrostatic pressure from 1 to 1500 bar with very precise pressure control (< 10 bar) on both increasing and decreasing pressure. This cell, in conjunction with the world-class instrumentation offered by Beamline I19, gives unparalleled access to the pressure region below the lowest pressures attainable using DACs, making it possible to investigate pressure responses for compounds that would otherwise have to be disregarded. 


\subsection{References}

1 Nowell, H., Barnett, S. A., Christensen, K. E., Teat, S. J. \& Allan, D. R. I19, the small-molecule single-crystal diffraction beamline at Diamond Light Source. Journal of Synchrotron Radiation 19, 435-441, doi:10.1107/s0909049512008801 (2012).

2 Bridgman, P. W. The Technique of High Pressure Experimenting. Proceedings of the American Academy of Arts and Sciences 49, 627-643, doi:10.2307/20025490 (1914).

3 Allan, D. R., Miletich, R. \& Angel, R. J. Diamond-anvil cell for single-crystal $\mathrm{x}$-ray diffraction studies to pressures in excess of $10 \mathrm{GPa}$. Review of Scientific Instruments 67, 840-842, doi:10.1063/1.1146820 (1996).

4 Definitions and symbols for units. Quantities, Units and Symbols in Physical Chemistry: Edition 3, 83-100, doi:10.1039/9781847557889-00083 (2007).

5 Weir, C., Block, S. \& Piermari.G. Single-crystal X-ray diffraction at high pressures. Journal of Research of the National Bureau of Standards Section C-Engineering and Instrumentation C 69, 275-281, doi:10.6028/jres.069C.032 (1965).

6 Weir, C. E., Lippincott, E. R., Vanvalkenburg, A. \& Bunting, E. N. Infrared Studies in the 1-Micron to 15-Micron Region to 30,000 Atmospheres Journal of Research of the National Bureau of Standards Section a-Physics and Chemistry 63, 55-62, doi:10.6028/jres.063A.003 (1959).

7 Graf, D. E., Stillwell, R. L., Purcell, K. M. \& Tozer, S. W. Nonmetallic gasket and miniature plastic turnbuckle diamond anvil cell for pulsed magnetic field studies at cryogenic temperatures. High Pressure Research 31, 533-543, doi:10.1080/08957959.2011.633909 (2011).

8 Ridley, C. J. \& Kamenev, K. V. High pressure neutron and X-ray diffraction at low temperatures. Zeitschrift Fur Kristallographie-Crystalline Materials 229, 171-199, doi:10.1515/zkri-2013-1673 (2014).

9 Bassett, W. A. Diamond anvil cell, 50th birthday. High Pressure Research 29, 163-186, doi:10.1080/08957950802597239 (2009). 
10 Piermarini, G. J. High pressure X-ray crystallography with the diamond cell at NIST/NBS. Journal of Research of the National Institute of Standards and Technology 106, 889-920 (2001).

11 Merrill, L. \& Bassett, W. A. Miniature diamond anvil pressure cell for single-crystal X-ray-diffraction studies. Review of Scientific Instruments $\mathbf{4 5}$, 290-294, doi:10.1063/1.1686607 (1974).

12 Moggach, S. A., Allan, D. R., Parsons, S. \& Warren, J. E. Incorporation of a new design of backing seat and anvil in a Merrill-Bassett diamond anvil cell. Journal of Applied Crystallography 41, 249-251, doi:10.1107/S0021889808000514 (2008).

13 Boehler, R. \& De Hantsetters, K. New anvil designs in diamond-cells. High Pressure Research 24, 391-396, doi:10.1080/08957950412331323924 (2004).

14 Van Valkenburg, A. Visual Observations of High Pressure Transitions. Review of Scientific Instruments 33, 1462-1462, doi:http://dx.doi.org/10.1063/1.1717803 (1962).

15 Piermarini, G. J., Block, S., Barnett, J. D. \& Forman, R. A. Calibration of pressure-dependence of $\mathrm{Rl}$ ruby fluorescence line to 195 kbar. Journal of Applied Physics 46, 2774-2780, doi:10.1063/1.321957 (1975).

16 Angel, R. J., Allan, D. R., Milletich, R. \& Finger, L. W. The use of quartz as an internal pressure standard in high-pressure crystallography. Journal of Applied Crystallography 30, 461-466, doi:10.1107/s0021889897000861 (1997).

17 Picard, A., Oger, P. M., Daniel, I., Cardon, H., Montagnac, G. \& Chervin, J.C. A sensitive pressure sensor for diamond anvil cell experiments up to 2GPa: FluoSpheres ${ }^{\circledR}$. Journal of Applied Physics 100, 034915, doi:http://dx.doi.org/10.1063/1.2234821 (2006).

18 Klotz, S., Chervin, J. C., Munsch, P. \& Marchand, G. L. Hydrostatic limits of 11 pressure transmitting media. Journal of Physics D: Applied Physics 42, 075413 (2009). 
19 Dawson, A., Allan, D. R., Parsons, S. \& Ruf, M. Use of a CCD diffractometer in crystal structure determinations at high pressure. Journal of Applied Crystallography 37, 410-416, doi:10.1107/S0021889804007149 (2004).

20 Parsons, S. ECLIPSE. (University of Edinburgh, 2004).

21 CrysAlis PRO (Agilent, Agilent Technologies Ltd, Yarnton, Oxfordshire, England., 2014).

22 SAINT v. 8.34A (Bruker AXS Inc., Madison, Wisconsin, USA, 2014).

23 APEX3 v. v2016.9-0 (Bruker AXS Inc., Madison, Wisconsin, USA, 2016).

24 SADABS v. 2008-1 ed. (Bruker AXS Inc., Madison, Wisconsin, USA, 2008).

25 Fourme, R. Appareillage pour etudes radiocristallographiques sous pression et a temperature variable. Journal of Applied Crystallography 1, 2330, doi:10.1107/S0021889868004954 (1968).

26 Oger, P. M., Daniel, I. \& Picard, A. Development of a low-pressure diamond anvil cell and analytical tools to monitor microbial activities in situ under controlled P and T. Biochimica et Biophysica Acta (BBA) - Proteins and Proteomics 1764, 434-442, doi:http://dx.doi.org/10.1016/j.bbapap.2005.11.009 (2006).

27 Girard, E., Dhaussy, A. C., Couzinet, B., Chervin, J. C., Mezouar, M., Kahn, R., Ascone, I. \& Fourme, R. Toward fully fledged high-pressure macromolecular crystallography. Journal of Applied Crystallography 40, 912-918, doi:10.1107/s0021889807033833 (2007).

28 Girard, E., Fourme, R., Ciurko, R., Joly, J., Bouis, F., Legrand, P., Jacobs, J., Dhaussy, A.-C., Ferrer, J.-L., Mezouar, M. \& Kahn, R. Macromolecular crystallography at high pressure with pneumatic diamond anvil cells handled by a six-axis robotic arm. Journal of Applied Crystallography 43, 762-768, doi:10.1107/S0021889810016146 (2010).

29 Vos, W. L. \& Schouten, J. A. On the temperature correction to the ruby pressure scale. Journal of Applied Physics 69, 6744-6746, doi:10.1063/1.348903 (1991).

30 Decker, D. L. High-Pressure Equation of State for $\mathrm{NaCl}, \mathrm{KCl}$, and $\mathrm{CsCl}$. Journal of Applied Physics 42, 3239-3244, doi:10.1063/1.1660714 (1971). 
31 Birch, F. Finite strain isotherm and velocities for single-crystal and polycrystalline $\mathrm{NaCl}$ at high pressures and $300^{\circ} \mathrm{K}$. Journal of Geophysical Research: Solid Earth 83, 1257-1268, doi:10.1029/JB083iB03p01257 (1978).

32 Hazen, R. M. \& Finger, L. W. Calcium fluoride as an internal pressure standard in high-pressure crystallography. Journal of Applied Crystallography 14, 234-236, doi:10.1107/S0021889881009266 (1981).

33 Angel, R. J. The high-pressure, high-temperature equation of state of calcium fluoride, CaF 2. Journal of Physics: Condensed Matter 5, L141 (1993).

34 Moury, R., Hauschild, K., Kersten, W., Ternieden, J., Felderhoff, M. \& Weidenthaler, C. An in situ powder diffraction cell for high-pressure hydrogenation experiments using laboratory X-ray diffractometers. Journal of Applied Crystallography 48, 79-84, doi:10.1107/S1600576714025692 (2015).

35 Cox, J. M., Walton, I. M., Benson, C. A., Chen, Y. S. \& Benedict, J. B. A versatile environmental control cell for in situ guest exchange singlecrystal diffraction. Journal of Applied Crystallography 48, 578-581, doi:10.1107/s160057671500432x (2015).

36 Bon, V., Senkovska, I., Wallacher, D., Heerwig, A., Klein, N., Zizak, I., Feyerherm, R., Dudzik, E. \& Kaskel, S. In situ monitoring of structural changes during the adsorption on flexible porous coordination polymers by X-ray powder diffraction: Instrumentation and experimental results. Microporous and Mesoporous Materials 188, 190-195, doi:http://dx.doi.org/10.1016/j.micromeso.2013.12.024 (2014).

37 Andrieux, J., Chabert, C., Mauro, A., Vitoux, H., Gorges, B., Buslaps, T. \& Honkimaki, V. A high-pressure and high-temperature gas-loading system for the study of conventional to real industrial sized samples in catalysed gas/solid and liquid/solid reactions. Journal of Applied Crystallography 47, 245-255, doi:10.1107/s1600576713030197 (2014).

38 Hill, A. A new gas system for automated in situ powder diffraction studies at the European Synchrotron Radiation Facility. Journal of Applied Crystallography 46, 570-572, doi:10.1107/S0021889813000629 (2013). 
39 Gray, E. M. \& Webb, C. J. In-situ diffraction techniques for studying hydrogen storage materials under high hydrogen pressure. International Journal of Hydrogen Energy 37, 10182-10195, doi:10.1016/j.ijhydene.2012.03.051 (2012).

40 Venturini, F., Schoder, S., Kuhs, W. F., Honkimaki, V., Melesi, L., Reichert, H., Schober, H. \& Thomas, F. A large-volume gas cell for high-energy X-ray reflectivity investigations of interfaces under pressure. Journal of Synchrotron Radiation 18, 251-256, doi:10.1107/S0909049510052106 (2011).

41 Jensen, T. R., Nielsen, T. K., Filinchuk, Y., Jorgensen, J. E., Cerenius, Y., Gray, E. M. \& Webb, C. J. Versatile in situ powder X-ray diffraction cells for solid-gas investigations. Journal of Applied Crystallography 43, 1456-1463, doi:10.1107/s0021889810038148 (2010).

42 Zhang, J.-P. \& Chen, X.-M. Optimized Acetylene/Carbon Dioxide Sorption in a Dynamic Porous Crystal. Journal of the American Chemical Society 131, 5516-5521, doi:10.1021/ja8089872 (2009).

43 Warren, J. E., Pritchard, R. G., Abram, D., Davies, H. M., Savarese, T. L., Cash, R. J., Raithby, P. R., Morris, R., Jones, R. H. \& Teat, S. J. A prototype environmental gas cell for in situ small-molecule X-ray diffraction. Journal of Applied Crystallography 42, 457-460, doi:10.1107/S0021889809010036 (2009).

44 Chupas, P. J., Chapman, K. W., Kurtz, C., Hanson, J. C., Lee, P. L. \& Grey, C. P. A versatile sample-environment cell for non-ambient X-ray scattering experiments. Journal of Applied Crystallography 41, 822-824, doi:10.1107/s0021889808020165 (2008).

45 Gonzalez, M. I., Mason, J. A., Bloch, E. D., Teat, S. J., Gagnon, K. J., Morrison, G. Y., Queen, W. L. \& Long, J. R. Structural characterization of framework-gas interactions in the metal-organic framework Co2(dobdc) by in situ single-crystal X-ray diffraction. Chemical Science 8, 4387-4398, doi:10.1039/C7SC00449D (2017). 
46 Tilton, R., Jnr. A fixture for X-ray crystallographic studies of biomolecules under high gas pressure. Journal of Applied Crystallography 21, 4-10, doi:10.1107/S0021889887008173 (1988).

47 Winter, G. xia2: an expert system for macromolecular crystallography data reduction. Journal of Applied Crystallography 43, 186-190, doi:10.1107/S0021889809045701 (2010).

48 Johnson, N. T. \& Probert, M. R. cbf_to_sfrm 2016 (alpha version), <https://github.com/nu-xtal-tools/cbf_to_sfrm> (2016). 
- Chapter 3 -

Sapphire Capillary Pressure Cell for In-Situ Single Crystal Diffraction Measurements on Beamline II9 at Diamond Light Source 


\subsection{Introduction}

Single-crystal and powder X-ray diffraction at kbar and Mbar pressures is now a well-established technique in chemical crystallography, driven by the availability of diamond anvil cells (DACs) that can be used on laboratory and synchrotron sources. ${ }^{1}$ As a high-pressure device the DAC is ubiquitous in the high-pressure field because of its simple desisgn and ease of use; however, it is not without its limitations. Two of the most significant limitations are that the supporting body for the anvils shadows a large volume of reciprocal space, leading to low completeness in all but the highest symmetry systems, and the lowest attainable pressures are usually in the region of 2000 to 3000 bar.

As the variety of compounds that scientists compress become larger and more sophisticated in their chemical architecture, their compressibility usually increases substantially, with crystalline samples becoming amorphous above 2000 bar or even less. ${ }^{2}$ These much larger, 'softer' compounds, therefore, become difficult to study at high-pressures as significant structural changes can occur well below the lowest attainable pressures when using a DAC.

Very few pressure cells have been developed for single-crystal X-ray diffraction in this moderate pressure region, which we define as being between 200 to 1500 bar. The earliest example is the Morosin \& Schirber cell (first described in 1965 but not in detail until 1974) that consisted of a capillary made of beryllium (Be) with a

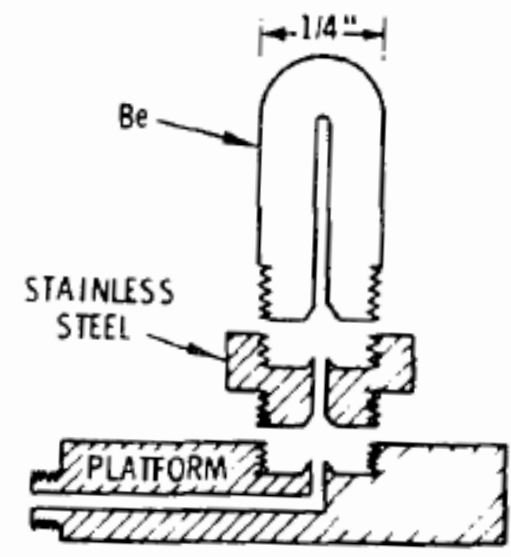

Figure 3.1 - A cross-section schematic of the Morosin \& Schirber cell. Image adapted from Morosin \& Schirber, 1974 
height of $19.05 \mathrm{~mm}$, an outer diameter (OD) of $6.36 \mathrm{~mm}$ and an inner diameter (ID) of $0.016 \mathrm{~mm}$, Figure 3.1..$^{3,4}$ The brittle Be capillary was permanently given a stainless steel ring, and metal-to-metal cone seals were used to allow the repeated disassembly of the cell to load samples without damage to the delicate Be threads. For low-temperature studies, the steel ring was replaced with niobium to better match the thermal expansion of Be. The sample crystal was glued to a glass fibre attached to a hollow metal pin. The crystal and pin were then inserted into the bore of the capillary to a known height to aid in the alignment of the crystal. This cell is reported to operate up to 4,000 bar and was used to investigate phase changes of ammonium fluoride and its deuterated equivalent along with the variation in electronic structures of antimony and bismuth. ${ }^{3,5}$

Moving forward the Kundrot \& Richards cell, closely based on the Morosin \& Schirber cell, was designed and published in 1986(Figure 3.2). ${ }^{6}$ This cell differed in several small ways, principally the dimensions of the capillary were changed to a much larger ID of $1 \mathrm{~mm}$ and a smaller OD of $5.5 \mathrm{~mm}$. The thinner wall thickness leads to this cell being rated to the lower pressure of 2,000 bar, while O-rings

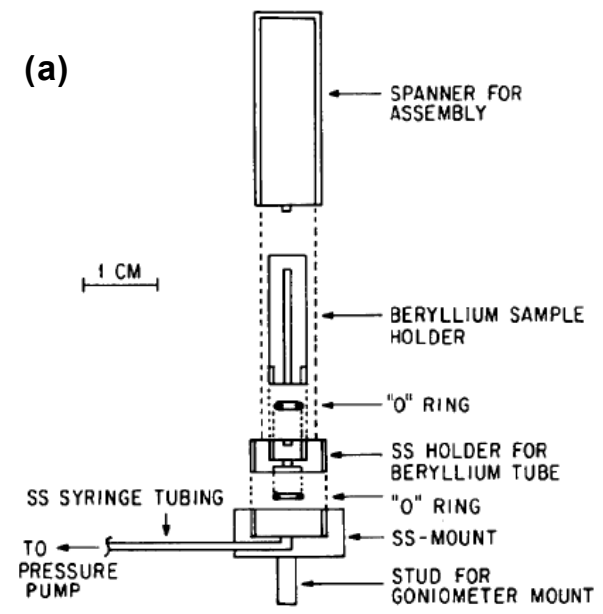

(b)

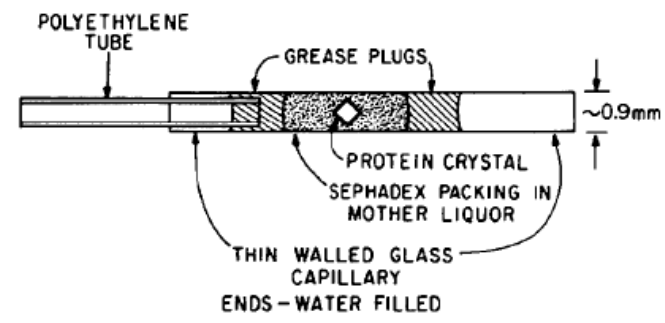

Figure 3.2 - (a) A cross-section schematic of the Kundrot \& Richards cell showing its construction. Note the inclusion of O-rings for the seals. (b) A cross-section schematic of the sample holder showing the crystal suspended inside a thin capillary between grease plugs. This whole assembly would be placed inside the beryllium sample holder. 
were used for the seals rather than metal-to-metal cone seals. The grade of Be used for this cell, I-440, had a known beryllium oxide (BeO) content of 4.2\%.

The larger internal diameter of this cell provided a novel opportunity to modify the sample-environment in which the crystal was held. This allowed Kundrot \& Richards to look at lysozyme protein crystals using the mother liquor as a hydrostatic medium while using an ethylene glycol/water (70:30\%) mixture as the pressurizing fluid. This was achieved by placing a single crystal into a thin-walled capillary suspended in polyacrylamide beads saturated in the mother liquor with grease seals at each end of the capillary. These seals provided a flexible barrier between the crystal and the pressurizing fluid allowing the crystal to be compressed in a small volume of mother liquor while the pressure was applied by a larger volume of ethylene glycol/water solution. A short length of polyethylene tubing was then used to ensure the crystal was at a known height in the capillary to aid with centring, as the sample was not visible due to the cell body.

In 2002 Urayama et al. reproduced the Kundrot \& Richards cell which was virtually unchanged apart from updated seals and a different grade of Be, I-250 (2.5\% BeO content). ${ }^{7}$ The lower $\mathrm{BeO}$ content improved X-ray transmittance without the loss of strength. This cell was used to study various protein systems

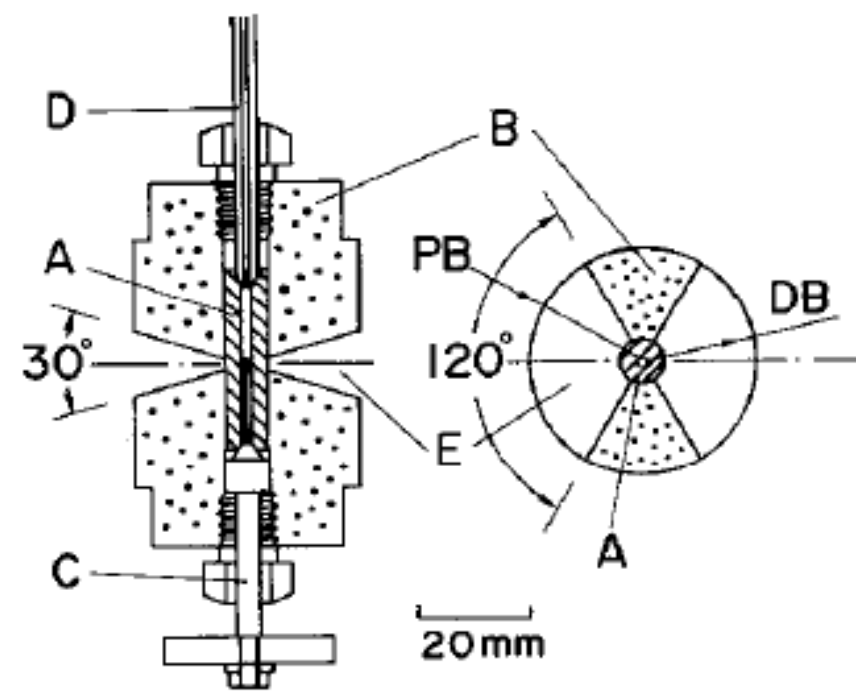

Figure 3.3 - A vertical and horizontal cross-section schematic of the Hamaya et al. cell. A beryllium cylinder, $B$ supporting jacket, $C$ sample holder, $\mathrm{D}$ pressure tubbing, $\mathrm{E}$ window, PB primary beam, DB diffracted beam. 
at Cornel University where the cell was developed. ${ }^{8,9}$ This same design was then reproduced by Suzuki et al., in 2010 with a slimmer Be capillary, $1.08 \mathrm{~mm}$ wall thickness, and further reduced $\mathrm{BeO}$ content $\mathrm{Be}(1 \% \mathrm{BeO}) .{ }^{10}$ This slimming of the wall thickness from the $2.25 \mathrm{~mm}$ present in the Kundrot \& Richards cell reduced the maximum working pressure to 1000 bar. This cell was made into a standalone unit that did not require a constant connection to a pump. In order to achieve this, they introduced a simple spring actuated pressure indicator along with a valve that can isolate the cell from the pump. This was used to investigate glucose isomerase using a rotating copper-anode lab source.

The last Be cell discussed is the Hamaya et al. cell that could achieve pressures as high as 5,000 bar using a supported Be capillary, Figure 3.3. ${ }^{11}$ The body of the cell was constructed from a copper-beryllium alloy and into this two windows were cut to expose the Be capillary. The windows expose $120^{\circ}$ in the horizontal and 2.4 $\mathrm{mm}$ in the vertical with an opening angle of $\pm 15^{\circ}$ to form an X-ray scattering plane. This design allows the pre-compression of the Be capillary by press fitting it into the body to allow the cell to access higher pressures that would otherwise be possible. The size and angles of the windows limit the accessible reciprocal space in a similar fashion to the shading in DAC's.

Be cells have dominated capillary pressure cells for diffraction studies due to their ability to transmit X-rays through the body of the cell. The cells are also ductile, strong and machineable. However, there are inherent drawbacks using Be-based capillaries. By visually obscuring the sample it can only be centred by diffraction, a time-consuming task. ${ }^{12}$ Be also causes intense Debye Scherrer rings on the diffraction images that hampers data processing. Another important consideration to safety is the toxicity of $\mathrm{Be}$ and $\mathrm{BeO}$ and the restrictions on handling and machining that this causes.

To overcome these drawbacks Yufit \& Howard proposed a simple pressure cell using a fused quartz capillary that mitigated some of the prime concerns of using Be, Figure 3.4. ${ }^{13}$ The transparent fused quartz capillary had an OD of $3 \mathrm{~mm}$ and an ID of $1 \mathrm{~mm}$ and was designed to withstand pressures up to $1 \mathrm{kbar}$. (Highest published pressure 550 bar ${ }^{14}$ ) However, the metal to quartz seal between the 
capillary and brass tube was a common site of failure, limiting the cells maximum operating pressure and reliability. The thickness of the amorphous capillary wall and the large ID caused a significant contribution to the background at low angle from diffuse scattering. This was, however still preferable to the intense Debye Scherrer rings found with Be. The setup allowed data to be collected on an inhouse diffractometer (Bruker Smart CCD 6000) with the cell mounted directly to the $\omega$-circle. During data collection, the cell was isolated from the pump and pressure gauge; therefore, in-situ pressure measurement or changes were not possible.
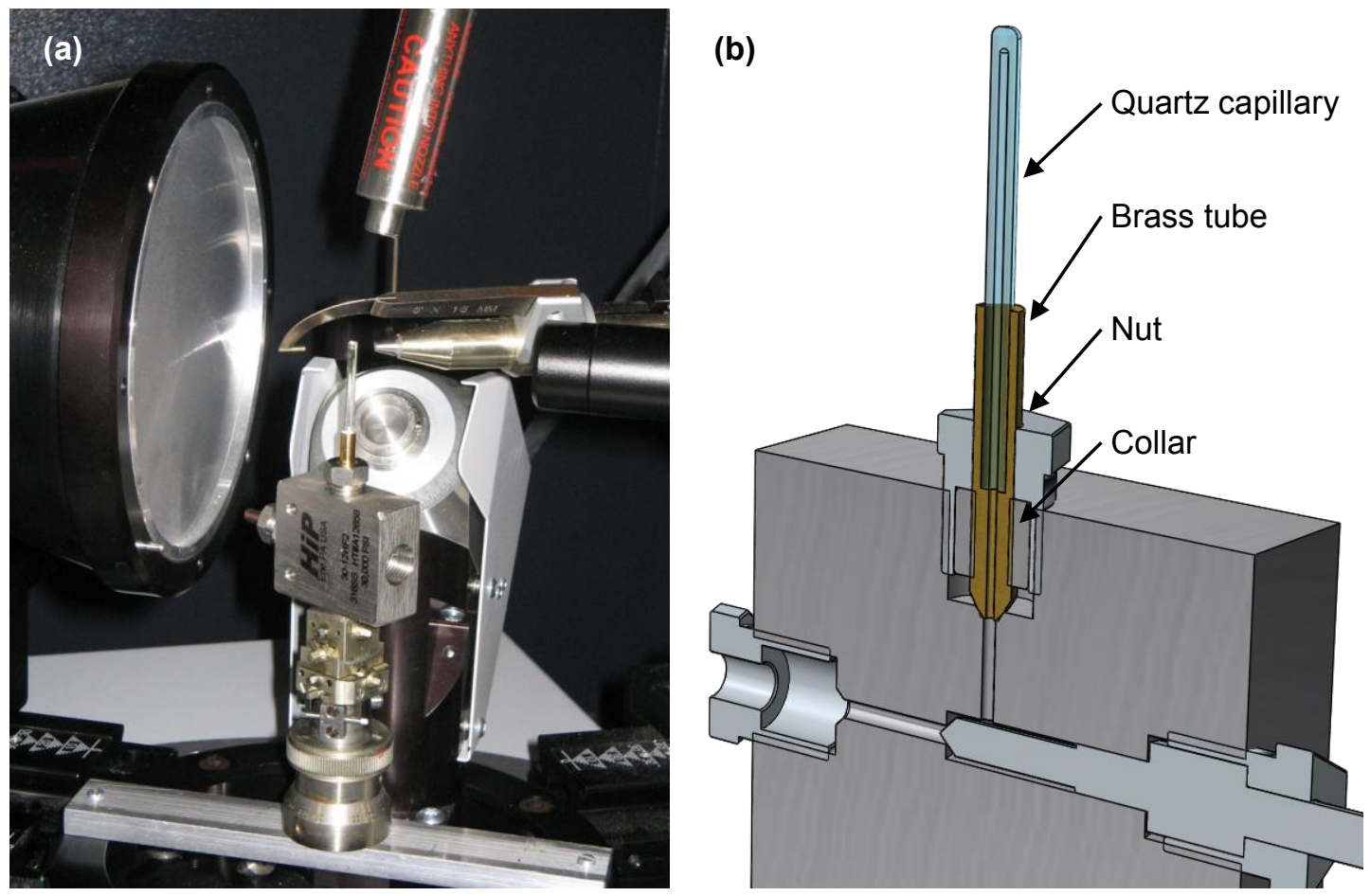

Figure 3.4 - (a) The Yufit \& Howard cell in place on the Bruker Smart CCD 6000 diffractometer at Durham University. Image courtesy of Dr D. Yufit. (b) A crosssectioned CAD model showing the construction of the cell. 
In this chapter, we present a new pressure cell design based around a sapphire capillary that has been optimised for the small molecule beamline I19 at Diamond Light Source. This cell allows access to pressures in excess of 1000 bar regularly with a maximum operating pressure of 1500 bar with very precise pressure control (< 10 bar) on both increasing and decreasing pressure. We have also developed a device that allows the sample to be compressed in a wide range of pressure transmitting medium. Here, the design, construction and method development of this new pressure cell and set-up will be discussed along with two compression studies on Hexamethylenetetramine (hexamine, $\mathrm{C}_{6} \mathrm{H}_{12} \mathrm{~N}_{4}$ ) and its deuterated form $\left(\mathrm{C}_{6} \mathrm{D}_{12} \mathrm{~N}_{4}\right)$, hereafter referred to as HMTH and HMTD respectively. The crystal structure of HMTH was first determined by Dickinson \& Raymond $\left(1923^{15}\right)$ and almost simultaneously by Gonell \& Mark $\left(1923^{16}\right)$, one of the first molecular crystal structures determined by X-ray crystallography. ${ }^{17}$ HMTH has been a model crystalline system for a variety of fundamental diffraction studies including charge density and neutron diffraction. ${ }^{18,19}$ Both HMTH and HMTD crystalize in the cubic space group $I \overline{4} 3 m$ which utilizes the full $\overline{4} 3 \mathrm{~m}$ symmetry to give an asymmetric unit consisting of three atoms, all of which lie on symmetry-constrained positions.

Here we present the compression behaviour and bulk moduli of both HMTH and HMTD up to a pressure of 1000 bar. These were used to assess the accuracy and precision of experiments performed using the new sapphire capillary pressure cell. We also report the previously unreported lattice parameters for HMTD. 


\subsection{Method Development}

This section covers the design, construction and method development for a new pressure cell for the small molecule beamline I19 at the Diamond Light Source. The aims for this cell at the outset were very clear:

- To enable single-crystal diffraction from ambient pressure to more than 1000 bar regularly with very precise pressure control (ca. 10 bar) both on increasing and decreasing pressure.

- Little or no restriction to available reciprocal space to allow the study of low symmetry systems.

- As a user facility, the cell would have to be simple, reliable, robust, and safe for all users.

- Integration with the existing instrumentation in EH2

A capillary cell design coupled to a high-pressure pump was determined to be the best solution building on and adapting tried and tested designs. 4,6,13,20 The pump allowed for precise pressure changes while a capillary cell can provide access to a large region of reciprocal space. A transparent capillary was favoured over a Be capillary as it would allow the sample to be centred optically while removing the toxic hazard of Be. Single-crystal synthetic sapphire, $\alpha-\mathrm{Al}_{2} \mathrm{O}_{3}$, was selected for the capillary material for its optical clarity, its strength, and availability.

All of these different elements were taken into consideration, and the result was the Sapphire Capillary Pressure Cell described in this chapter. 


\subsubsection{Sapphire capillary pressure cell design}

The design of the sapphire capillary pressure cell consists of three distinct elements:

- The pressure cell - This is where the sample was contained

- The phase separator - This connected the cell to the instrument and linked it to the pump.

- The high-pressure hand pump - This generated the pressure that was applied to the sample in the pressure cell.

Each of these elements will be discussed in detail in the following section along with the integration of the whole set up on beamline I19, EH2, at the Diamond Light Source.

\subsubsection{Construction of the sapphire capillary pressure cell}

The high-pressure sample environment was made using a sapphire capillary fixed into a holder. The synthetic sapphire capillaries were obtained from CRYTUR, spol. s r.o. (Czech Republic) in two different sizes. The larger capillaries measured $1.4 \mathrm{~mm}$ OD x $1.0 \mathrm{~mm}$ ID while the smaller measured $1 \mathrm{~mm}$ OD x $0.6 \mathrm{~mm}$ ID. This gave a wall thickness of $0.2 \mathrm{~mm}$ for both sizes. All the capillaries were supplied

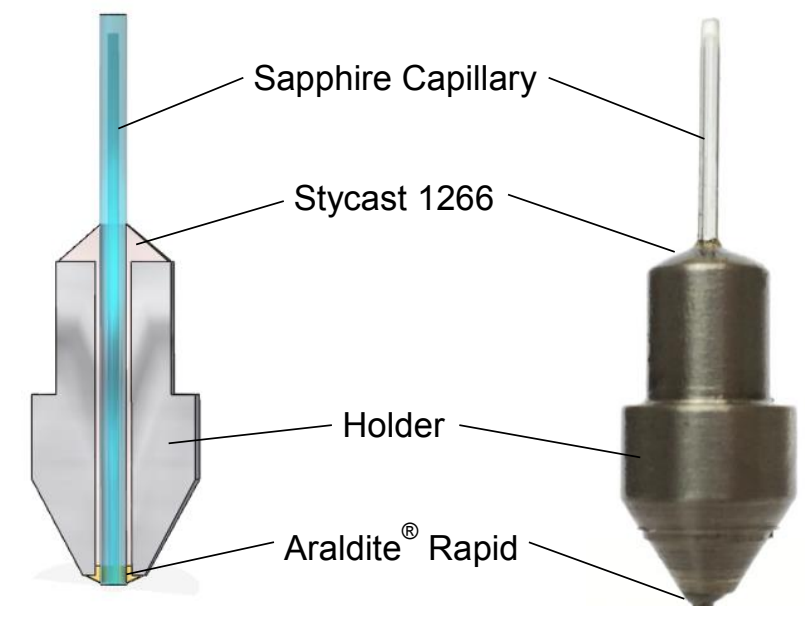

(a)

(b)

Figure 3.5 - (a) A sectioned CAD model showing the deep and narrow glue gap between the holder and the sapphire capillary that is filled by the Stycast 1266. (b) An image of a completed $1 \mathrm{~mm}$ OD x $0.6 \mathrm{~mm}$ ID sapphire capillary pressure cell. 
with a single end closed and measured $30 \mathrm{~mm}$ in length. The sapphire was provided with an 'as grown' finish as the capillaries were too fragile to polish.

Prior to the construction of the pressure cell, the sapphire capillaries were carefully inspected for flaws, scratches or micro-cracks that could lead to premature failure in service. The pressure cell was manufactured from a stainless steel holder in which the capillaries were glued into. The holder, Figure 3.5, was made to interface with existing pressure rated equipment through a cone type metal-to-metal seal which allowed integration into the rest of the pressure apparatus. For this, the male cone of the holder was machined to $60^{\circ}$ to form a seal with the $58^{\circ}$ female cone of the phase separator. This was then sealed using a modified gland nut (Figure 3.6).

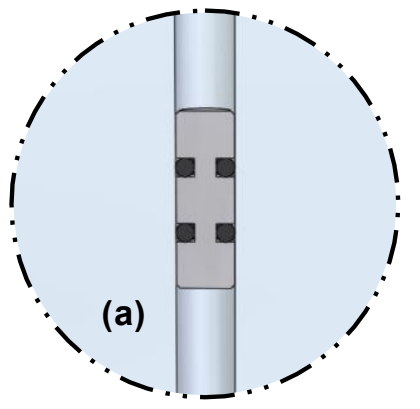

$2: 1$

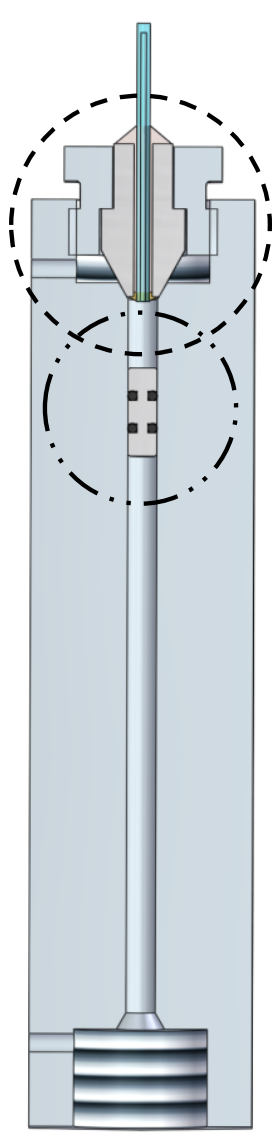

$1: 1$

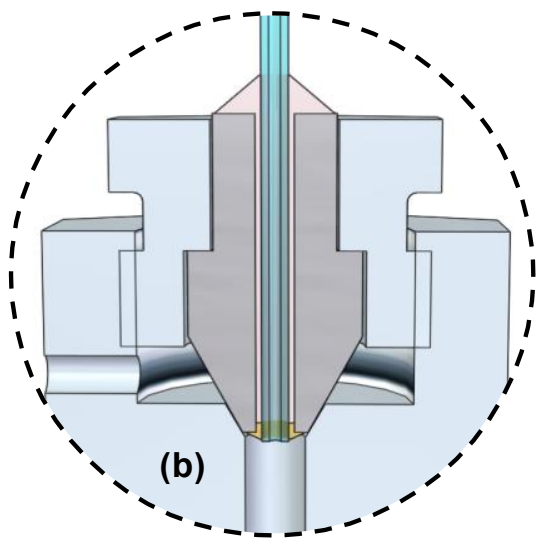

2:1

Figure 3.6 - A 1:1 scale CAD model of the phase separator with 2:1 inserts: (a) The movable piston with double O-rings; (b) The cone type metal-to-metal seal between the holder of the cell and the phase separator body. 
The ID of the holder is specifically oversized compared to the capillary to allow the glueing process to be achieved. In this case, all holders used had an ID of 2 $\mathrm{mm}$. The primary adhesive used was Stycast 1266 (Henkel Loctite) epoxy. The internal bore of the holder was polished to remove imperfections from machining and thoroughly degreased and cleaned. The capillary was then inserted into the holder and initially glued at the base of the holder using a standard quick setting epoxy (Araldite ${ }^{\circledast}$ Rapid). As the epoxy cured, the capillary was kept central and straight in the bore of the holder to make the glue gap equidistant around the capillary. The open end of the capillary extended past the base of the holder by only $1-2 \mathrm{~mm}$. This epoxy plug was not structural but merely held the capillary in place for the next stage of the glueing process.

The Stycast 1266 two-part epoxy was prepared to the manufacturer's recommendations including thorough degassing under vacuum to remove any adsorbed or entrapped air from mixing. With the holder and capillary standing vertically the Stycast was fed into the gap between the two. The low viscosity of the Stycast allowed it to flow down into the workpiece. Air entrapment is inevitable as the gap is narrow and deep. The whole cell was placed under vacuum to draw any trapped air out and entirely fill the gap. As the cell was returned to ambient pressure, the epoxy was drawn deeper into the gap. More epoxy was then fed into the gap and then placed under vacuum again. This was an iterative process until the gap had been filled with epoxy. The degassing process was vital to achieve a good and reliable seal. To finish, a fillet between the capillary and holder was formed using a small excess of epoxy. This lessened the collaring effect and helped smooth the pressure gradient between supported and unsupported capillary. The cell was then left to cure at room temperature for 16 hours followed by a further two hours at $100^{\circ} \mathrm{C}$.

After this process, each cell was subjected to an initial pressure test to remove any cells that were defective from the manufacturing process and to give a maximum working pressure of each individual cell. The safety protocol for pressure testing of the cells is described below, §3.2.5.1. 


\subsubsection{Phase separator}

The phase separator was custom built elongated female union with a $3.15 \mathrm{~mm}$ central bore that the cell was attached too. In the central bore, a piston with double O-rings acted as a movable physical barrier to keep the hydraulic fluid from the pump separate from the hydrostatic media selected to compress the sample, Figure 3.6. This device allows compression of the sample with a wide variety of hydrostatic medium, and quickly change between them. ${ }^{21}$ Completely full, the phase separator holds $0.53 \mathrm{ml}$ of compression media, however, it was rarely necessary to use the entire volume of the cell, as any trapped air in the capillary could be easily dissolved upon increasing pressure.

\subsubsection{High-pressure hand pump}

To generate the pressure, a custom setup was designed and built using commercially available parts, Figure 3.7. The pump selected was an ENERPAC P2282 (supplied by Norcam Hydraulic Services Ltd, UK) a two-stage portable hand pump rated to 2800 bar. To measure the pressure, a digital transducer was used (DMP 304, Impress Sensors \& Systems, UK) which was rated to 4000 bar and

(a)

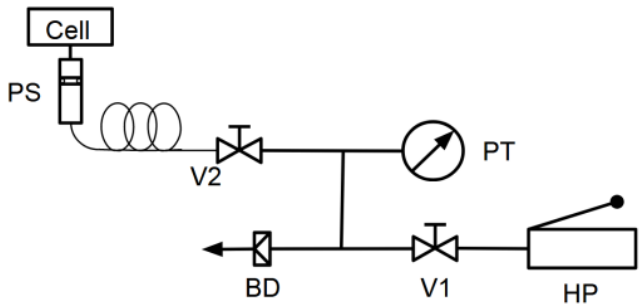

\begin{tabular}{c|c} 
Name & Description \\
\hline HP & Hand pump \\
V1 & Metering Valve \\
V2 & Lock off Valve \\
BD & Burst Disc \\
PT & Pressure Transducer \\
PS & Phase Separator
\end{tabular}

(b)

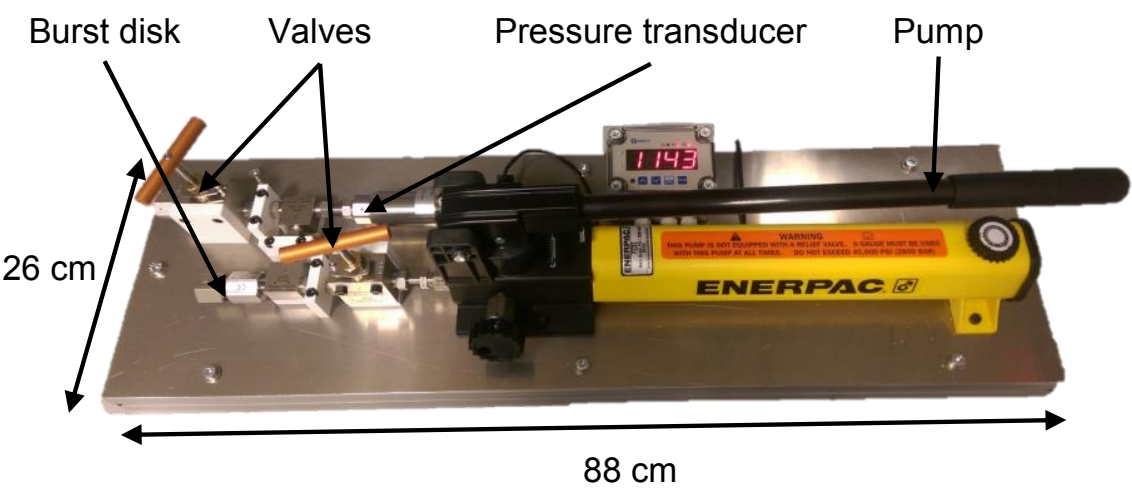

Figure 3.7 - The high pressure hand pump set up: (a) Schematic of the set up with a parts list; (b) Image of the portable set up in use, note the pressure read out on the digital process indicator of 1143 bar. 
Chapter 3 - Sapphire Capillary Pressure Cell for In-Situ Single-Crystal Diffraction

Measurements on Beamline I19 at Diamond Light Source

accurate to $0.5 \%$ full-scale output (FSO). To provide a visual readout of the pressure, this was coupled to a digital process indicator (Simex, SRP-N118) and integrated into the Experimental Physics and Industrial Control System (EPICS) custom software environment to allow the pressure to be logged and monitored in real time from the control room on station I19. All additional parts were manufactured by SITEC-Sieber Engineering AG (supplied by Hi-Pro Pressure Products Ltd., UK) with 1/4" parts rated to 4000 bar. A burst disk was fitted as a prevention to an over pressurisation of the pump and components. The whole setup was mounted onto a portable trolley so that it could be moved around the beamline. The pump was connected to the phase separator by a 1116 " high-pressure tubing rated to 2000 bar. 


\subsubsection{Integration into EH2}

Beamline I19 consists of two experimental hutches and an optics hutch, Figure 3.8. $\mathrm{EHl}$ is closer to the storage ring and is dedicated to high throughput small molecule single-crystal diffraction. EH2 is adaptable to a number of different sample environment setups such as high-pressure diamond-anvil cells, variable temperature open-flow and closed-cycle cryostats, and gas exchange using a gas cell described in Chapter 2, \$2.2. Some features of these setups are semipermanent fixtures in the hutch however the majority need to be able to be fitted and removed for each experiment. The setup on I19 allows more complex set-ups to be installed or removed from EH2 while the beamline is still operational.

The focal point of EH2 is the large 4-circle Newport goniometer that is equipped with a DECTRIS PILATUS 300K hybrid-pixel detector. Due to its size, this goniometer permits considerable freedom when mounting different sample environments and is able to support up to $25 \mathrm{~kg}$ at the diffraction position.

To mount the sapphire capillary pressure cell to the goniometer, a 3D printed mount was made to hold the phase separator securely in the diffraction position, Figure 3.9. The mount was bolted directly to the $\varphi$-circle. A motorised XYZ goniometer head allowed centring of the cell to be performed remotely. Despite the goniometer being able to support large loads, the mount was kept as rigid and as lightweight as possible to minimise the effect on the sphere of confusion.

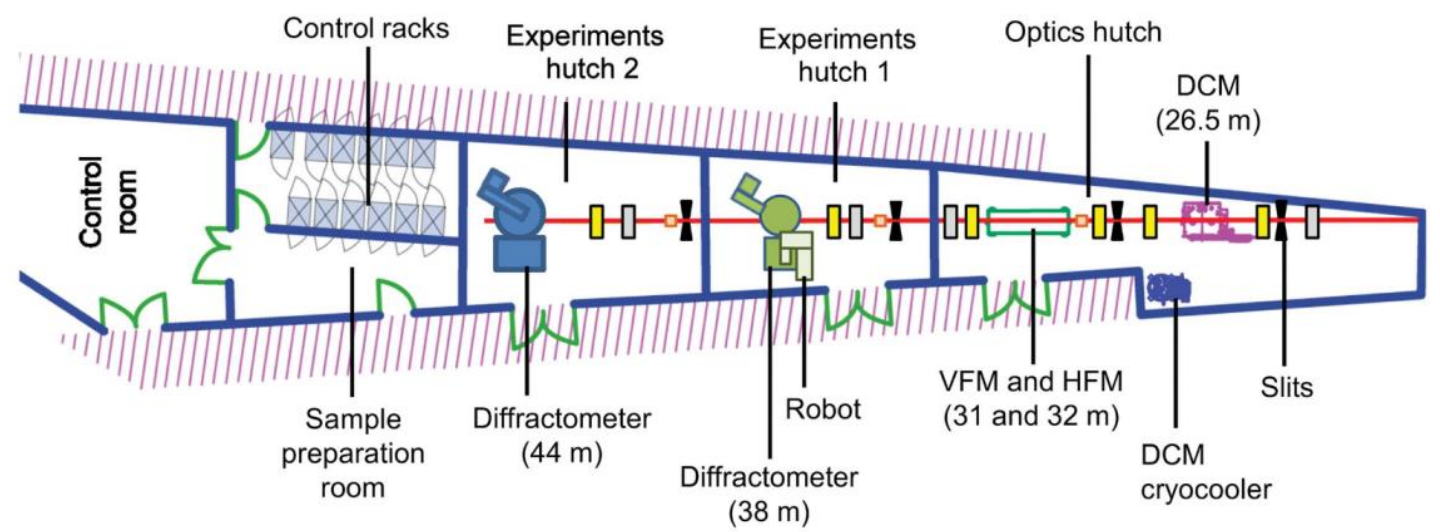

Figure 3.8 - Layout of beamline I19, showing the two experimental hutches in which the single-crystal diffraction experiments are carried out. 
The phase separator was connected to the pump via a length of $1 / 16$ " high-pressure tubing that was routed through the centre of the $\varphi$ axis. In this configuration, the rotation of $\omega$ and $\varphi$ were not hampered by the tubing. However, due to the tight space between $\kappa$ and $\omega, \kappa$ was restricted to $\omega$-values between $45^{\circ}$ and $0^{\circ}$. The highpressure tubing was then routed under the hutch floor and through a chicane into the sample preparation room where the portable high-pressure hand pump was set up, Figure 3.8. This allowed pressure changes to be made while the hutch was interlocked speeding up data collection and adding a layer of protection to the user by removing them from the vicinity of the pressurised sapphire capillary cell. In total there were $8 \mathrm{~m}$ of high-pressure tubing between the pump set-up and the phase separator. Despite this, pressure transfer was found to be virtually instantaneous in tests with two pressure transducers, one at the pump and another at the phase separator.

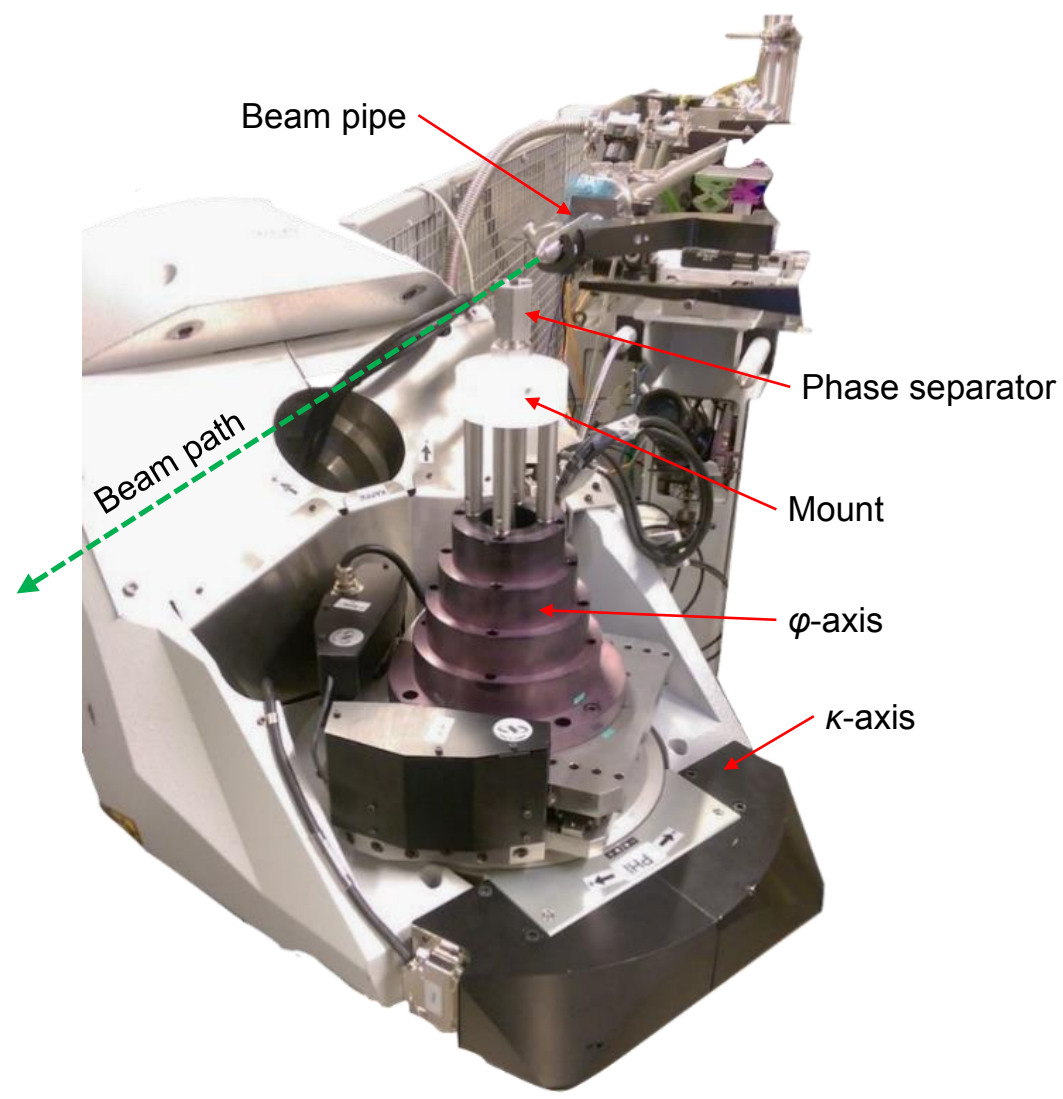

Figure 3.9 - The mount for the phase separator on the 4-axis Newport goniometer in $\mathrm{EH} 2$, only $\varphi$ and $\kappa$ are visible. This vertical geometry is used for attaching the cell to the phase separator and optical alignment. 


\subsubsection{Collecting single-crystal X-ray diffraction data}

\subsubsection{Single-crystal mounting}

Single-crystals were mounted inside the capillary cell in a variety of ways. The simplest and most commonly used method was to mount a single-crystal to the tip of a carbon fibre rod or PVC fibre using a minimum amount of cyanoacrylate adhesive (Figure 3.10), which was particularly important when investigating interactions between the sample and pressurising fluid. The mounted sample was then inserted into the sapphire capillary cell where it was held in place with a small amount of adhesive at the opening of the capillary, sticking the fibre to the capillary wall, taking great care not to seal the opening of the capillary.

\subsubsection{Sample alignment}

On the goniometer, the crystal was centred optically, a major advantage to using a transparent capillary. In certain circumstances, especially when investigating small $(<100 \mu \mathrm{m})$ transparent samples, accurate optical centring could be challenging due to optical distortions from the capillary walls (Figure 3.10). Filling the capillary with a very low pressure of the hydrostatic fluid ( $<10$ bar) could reduce the distortions if the refractive index of the hydrostatic fluid was similar to that of sapphire $\left(n_{o}=1.768 n_{e}=1.760\right.$ at $\left.589.29 n m\right)$.

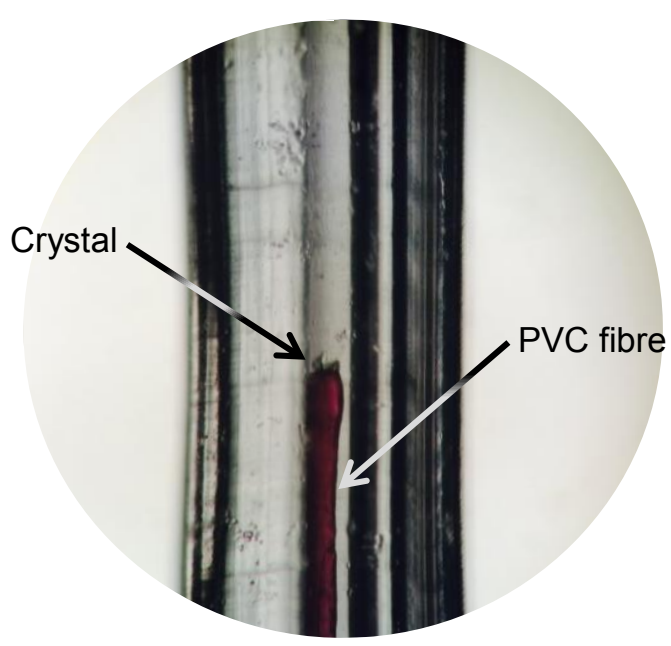

Figure 3.10 - The view of a $100 \mu \mathrm{m}$ crystal during centring. The texture of the as grown finish of the sapphire capillary is clearly visible. 
In addition to optical centring, centring by diffraction was used to align the sample. This was achieved by collecting an initial dataset, then during data reduction, the direction and distance of the crystal from the diffraction centre (the crystal offset from the direct beam centre) were derived. Once the offsets were determined, the motorized goniometer head was used to re-centre the crystal. This has been shown to be a robust method for aligning samples contained inside a displex low-temperature device (which has a Be-shroud), where the sample cannot be aligned (or seen) optically. ${ }^{12}$ This can also be used to monitor small changes in the crystal position as the pressure is increased.

\subsubsection{Collection strategy}

The collection strategy developed for the sapphire capillary pressure cell consisted of a series of $\omega$-scans at three settings of $\varphi$ and two additional $\varphi$ scans, Table 3.1. This minimised the time for data collection while at the same time ensuring significant coverage of reciprocal space for compounds that crystallise with medium-sized (5-20 A) unit cell dimensions. The detector was set to $30^{\circ}$ (20) with a detector distance of $85 \mathrm{~mm}$. This strategy gave data to a resolution of $0.68 \AA$ (at $\lambda=0.6889 \AA)$.

A single data collection takes 12 minutes 40 seconds, using this strategy; however, with pressure increases and repositioning of the goniometer, this time could increase to approximately 15 minutes.

Table 3.1 - Summary of the data collection strategy used for the sapphire capillary pressure cell.

\begin{tabular}{cccccccc}
\hline $\begin{array}{c}\text { Scan circle } \\
(\boldsymbol{\varphi} / \boldsymbol{\omega})\end{array}$ & $\begin{array}{c}\text { Other } \\
\text { axis }\left({ }^{\circ}\right)\end{array}$ & $\boldsymbol{K}\left({ }^{\circ}\right)$ & $\mathbf{2 \theta}\left({ }^{\circ}\right)$ & Scan $\left(^{\circ}\right)$ & Width $\left(^{\circ}\right)$ & Time $(\mathbf{s})$ & $\begin{array}{c}\text { Detector } \\
\text { Distance }(\mathbf{m m})\end{array}$ \\
\hline$\omega$ & -120 & -45 & 30 & -156 to -30 & 0.2 & 0.2 & 85 \\
$\varphi$ & -30 & -45 & 30 & -120 to 0 & 0.2 & 0.2 & 85 \\
$\omega$ & 0 & -45 & 30 & -156 to -30 & 0.2 & 0.2 & 85 \\
$\varphi$ & -30 & -45 & 30 & 0 to 120 & 0.2 & 0.2 & 85 \\
$\omega$ & 120 & -45 & 30 & -156 to -30 & 0.2 & 0.2 & 85 \\
\hline
\end{tabular}




\subsubsection{Calibration of instrument}

A well-calibrated instrument and accurate instrument model was essential for tracking very small unit cell changes and accurate centring by diffraction. Powder diffraction from Lanthanum hexaboride $\left(\mathrm{LaB}_{6}\right)$ at three different detector distances with $2 \theta=0^{\circ}$ was used to determine two key instrument parameters, the beam centre and detector distance. ${ }^{22}$ Following this, a well-defined standard, such as ruby or yild, was run to define the remaining goniometer and detector parameters. This instrument model was then used during data reduction. Attenuation and exposure times were adjusted sample-to-sample to keep the photon count rate to the detector within its linear region ( $>20 \%$ of maximum count rate).

\subsubsection{Wavelength selection}

Wavelength selection is a fine balance between several competing factors. Harder $\mathrm{X}$-rays will reduce absorption from the sapphire capillary; however, the diffracted intensities will be reduced (intensity is proportional to $\lambda^{3}$ ). The efficiency of the detector is also reduced at shorter wavelengths.

Linear absorption coefficients $(\mu)$ for $\mathrm{Al}_{2} \mathrm{O}_{3}$ were calculated for two of the most common edges used on I19, Ag and Zr, Table 3.2. The transmitted intensity (I) was calculated from the initial intensity $\left(\mathrm{I}_{0}\right)$; the total wall thickness $(\mathrm{x})$ of the capillary, $0.4 \mathrm{~mm}$; and the linear absorption coefficient at a given energy using: $I=I_{0} e^{-\mu x}$.

Table 3.2 - Absorption profiles for both $\mathrm{Zr}$ and Ag radiation.

\begin{tabular}{c|cc} 
& $\mathbf{Z r}$ & $\mathbf{A g}$ \\
\hline Wavelength (A) & 0.6889 & 0.4859 \\
Energy (keV) & 18.0 & 25.5 \\
$\boldsymbol{\mu} \mathrm{Al}_{2} \mathrm{O}_{3}(\mathbf{m m}-\mathbf{1})$ & 0.883 & 0.289 \\
$\mathrm{I}$ where $\mathrm{x}=\mathbf{0 . 4} \mathbf{~ m m}$ & $70.2 \%$ & $89.1 \%$
\end{tabular}

Despite the transmitted intensity being higher for $\mathrm{Ag}$ radiation, $\mathrm{Zr}$ was selected as the reduction in transmittance was negated by the greater intensity of the diffracted X-rays at this wavelength and the greater flux from the monochromator 
Chapter 3 - Sapphire Capillary Pressure Cell for In-Situ Single-Crystal Diffraction

Measurements on Beamline I19 at Diamond Light Source

at the $\mathrm{Zr}$-edge. However, for a highly absorbing sample, or a capillary with a greater wall thickness, $\mathrm{Ag}$ radiation could potentially be more suitable.

\subsubsection{Pressure equilibration}

In DAC experiments leaving equilibration time between data collections is important as the pressure can continue to increase after the pressure is increased. In diffraction experiments using the sapphire capillary cell, once the pressure was increased, any changes in pressure were assumed to be instantaneous on the time scale of the data collection. This is in part due to the pressure increases typically being 20-30 times smaller than those made during DAC experiments (100 bar vs 3000 bar) and that the pressure is applied hydraulically to the pressure vessel with no moving parts, unlike in a DAC. 


\subsubsection{Data reduction}

Processing the diffraction images was relatively straightforward compared to DAC experiments, as the sapphire cell does not shade the detector (as a DAC does) while parasitic Debye-Scherrer rings from the gasket material were also not present. However, the background was variable due to intense but broad reflections from the crystalline capillary itself, Figure 3.11. Some of the capillaries were composed of just a single-component, but others could contain up to five domains.

The Dectris Pilatus 300K hybrid-pixel detector outputs frames in the crystallographic binary format, .cbf. These can be directly fed into the data reduction program CrysAlisPro. ${ }^{15}$ Specific user input is required at each stage: indexing the unit cell, integration, absorption correction, and space group determination. APEX3, another data reduction software package produced by Bruker can also be used; however, the frames have to be converted from .cbf to a Bruker proprietary format, .sfrm. ${ }^{23,24}$ To convert the frames the program CBF2SFRM can be used. ${ }^{25}$

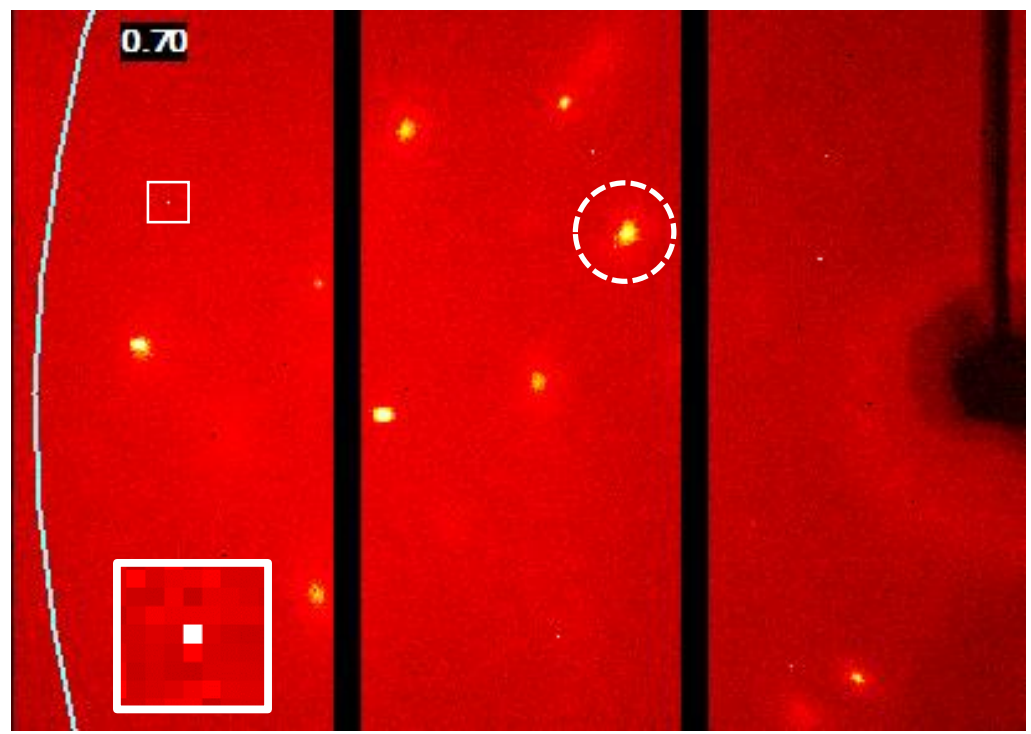

Figure 3.11 - A typical frame during collection from the Dectris Pilatus $300 \mathrm{~K}\left(0.2^{\circ}\right.$, $0.2 \mathrm{~s}$ ). Note the large and broad reflections caused by the sapphire capillary, white dashed circle. The insert shows a magnified peak from the sample. 
Chapter 3 - Sapphire Capillary Pressure Cell for In-Situ Single-Crystal Diffraction

Measurements on Beamline I19 at Diamond Light Source

With recent developments, the automatic data reduction software Xia2 can now handle the multicomponent nature of the diffraction patterns. ${ }^{26}$ Xia2 is developed in-house at Diamond Light Source with the aim of increasing the speed and, crucially, in this case, reducing the amount of user input required to reduce the large quantities of data collected.

In all cases, it was found that for accurate tracking of very small changes observed with small increments of pressure, the instrument must be extremely well calibrated and stable over the duration of the experiment. The key to reliable processing is the use of a precise instrument model determined prior to the experiment, and all data refined against the same model. 


\subsubsection{Safety Protocol}

Conducting experiments safely on the beamline required built-in safety features and a safe system of work. The high pressures involved in these experiments pose a hazard to both the user and the equipment; however, the risk of harm or damage has been minimised as far as possible.

These issues are particularly important, as this cell was designed to be a user facility. A safe system of work was therefore developed. In accordance with protocols at the Diamond light source, a number of additional safety features that were implemented in the design are described below.

\subsubsection{Pressure rating for the sapphire cell and pump-system}

All the commercially available components used have maximum operating pressure of 2000 bar or above. As the pump does not have its own safety release valve, a burst disk was included in the high-pressure side of the setup to prevent accidental over-pressurisation. This disk was rated to 2500 bar as per the manufactures instructions, stating the maximum operating pressure (2000 bar) should not exceed $80 \%$ of the nominal burst pressure.

The sapphire capillary was the most likely point of failure of the entire cell. Calculating safe working pressures was very challenging due to the brittle nature of the sapphire, and so each pressure cell was pressure tested on a separate rig to give a maximum working pressure of each cell. In use, the pressure during an experiment should not exceed $80 \%$ of the maximum working pressure. Therefore a cell with a maximum working pressure of 1250 bar should not be used above 1000 bar and so on.

\subsubsection{Cell failure protection}

Catastrophic failure of the sapphire capillary cell body was identified as the greatest hazard to the user and equipment during data collection. If the cell did fail while pressurised, it would do so catastrophically with no prior indication. Upon failure, the cell disintegrates into extremely small shards of sapphire and a plume of the aerosolised hydrostatic medium. 
To eliminate the risk to users, the setup was designed to remove the user from the vicinity of the cell while it was pressurised. The high-pressure hand pump (§3.2.1.3) was situated in the sample preparation room outside of the experimental hutch, and local rules prohibited the pressurisation of the system without the experimental hutch being interlocked. As an extra precaution, while operating the high-pressure hand pump, users were required to use a full-face shield.

The risk to equipment inside the hutch cannot be mitigated by removal. It was, therefore, necessary to reduce the stored energy to a minimum to reduce the risk of damage to the instrument, in particular, the detector. By using the phase separator, the majority of the liquid under pressure was a highly incompressible hydraulic fluid, significantly reducing the stored potential energy. The detector was by far the most vulnerable piece of equipment due to its close proximity to the cell during data collection, $85 \mathrm{~mm}$. To protect the detector a Kapton( polyimide film, $75 \mu \mathrm{m}$ thick, was affixed to the face. 


\subsection{Experimental}

\subsubsection{Sample preparation}

Single crystals of hexamethylenetetramine $\left(\mathrm{C}_{6} \mathrm{H}_{12} \mathrm{~N}_{4}\right)$, HMTH were used as supplied from Sigma Aldrich without recrystallization (CAS Number: 100-97-0, $\geq$ $99.0 \%)$.

Single crystals of the deutero-hexamethylenetetramine $\left(\mathrm{C}_{6} \mathrm{D}_{12} \mathrm{~N}_{4}\right)$, HMTD were used as supplied from CND Isotopes, purchased from Qmx Laboratories, without recrystallization (CAS Number: 23304-08-7, $99.9 \%$ atom D).

\subsubsection{Laboratory single-crystal measurements}

Ambient temperature and pressure single-crystal data were collected for HMTD on a three-circle Bruker SMART APEXII diffractometer with graphite monochromated $\mathrm{MoK}_{\alpha}$ radiation $(\lambda=0.71073 \AA)$. The data were collected at 297 $\mathrm{K}$, under a stream of nitrogen. ${ }^{27} \mathrm{~A}$ refined strategy was used to yield a complete, and highly redundant data set (redundancy $=26.4$ out to $0.8 \AA$ ). Unit cell indexing and data processing were carried out using the Bruker APEX3 software. Integration was performed using the program SAINT while the absorption corrections were carried out using the program SADABS. ${ }^{28,29}$

Structure refinements were carried out in CRYSTALS. ${ }^{30}$ The structure was refined anisotropically against $F^{2}$ with an $\mathrm{I} / \sigma$ cut-off set to -3.00 and a $[\sin \theta / \lambda]^{2}$ of 0.01 . The deuterium atom was placed geometrically and constrained to ride the carbon atom.

\subsubsection{Synchrotron single-crystal moderate pressure measurements}

Single crystals of both HMTH and HMTD were selected and mounted into separate sapphire capillary cells following the procedure outlined above, §3.2.3.1. The hydrostatic medium was a high bulk modulus hydraulic oil (ENERPAC HFSeries). All the high-pressure data sets were collected at room temperature (297 $\mathrm{K})$. Measurements were taken at ambient pressure and then in 100 bar steps up to a maximum pressure of 1000 bar. Measurements were also made on decreasing pressures in 200 bar steps back to ambient pressure. 
Chapter 3 - Sapphire Capillary Pressure Cell for In-Situ Single-Crystal Diffraction

Measurements on Beamline I19 at Diamond Light Source

Diffraction data using the sapphire capillary pressure cell were collected on beamline I19, Experimental Hutch 2 (EH2) at the Diamond Light Source, Rutherford Appleton Laboratory. EH2 is equipped with a Newport 4-circle goniometer with a DECTRIS PILATUS 300K hybrid-pixel detector using synchrotron radiation $(\lambda=0.6889 \AA)$. Data collection was carried out using an exposure time and a step size of 0.2 second and 0.2 degrees respectively using the strategy outlined above, \$3.2.3.3. CrysAlisPro (version: 171.38.46) was used for integration, absorption correction and space group determination.

Structure refinements were carried out in CRYSTALS. ${ }^{30}$ All structures were refined anisotropically against $F^{2}$. The hydrogen and deuterium atoms were placed geometrically and constrained to ride the carbon atom. 


\subsection{Results and Discussion}

\subsubsection{Ambient pressure structures of HMTH and HMTD}

Before experiments at pressure were conducted, it was important to confirm that data collected at ambient pressure from crystals inside the cell matched literature sources, and data collections from the same sample collected under ambient conditions (on a fibre). This provided a test for the instrument calibration, sample centring protocols and develop the best methods for data reduction.

The literature lattice parameters for $\mathrm{HMTH}$, under ambient pressure and temperature conditions were taken from the high-resolution X-ray study by Stevens \& Hope, 1975, ( $a=7.028(2) \AA$, collected with MoK $\alpha$ radiation). ${ }^{31}$ Here, measurements were conducted at ambient pressure and temperature on beamline I19, EH2, using synchrotron radiation $(\lambda=0.6889 \AA)$ with a single-crystal inside the sapphire capillary cell. The resulting measurements gave $a=7.0266(4) \AA$ and $a=7.0278(4) \AA$ before and after decompression back to ambient pressure, respectively, both of which are within error of the literature value given by Stevens \& Hope.

Literature values for the lattice parameters of HMTD under ambient pressure and temperature were not available. Therefore two additional measurements at ambient pressure and temperature were conducted on separate crystals and

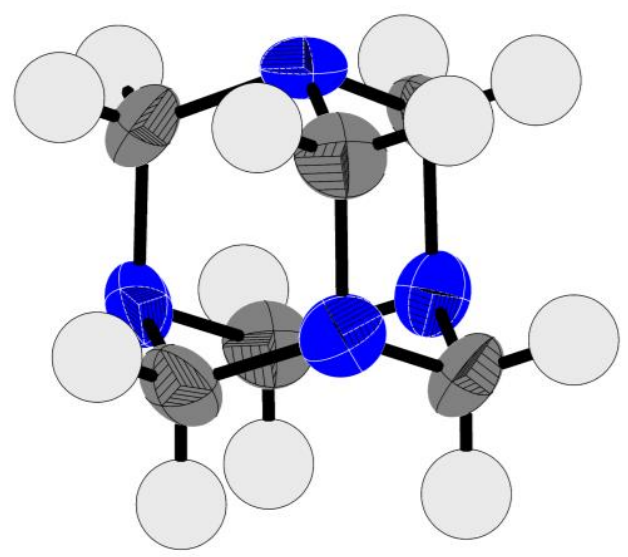

Figure 3.12 - The structure of HMTH at ambient pressure. Ellipsoids set at $50 \%$ probability. $\mathrm{C}$-atoms in grey, $\mathrm{N}$-atoms in blue, and $\mathrm{H}$-atoms in white. 
different instruments. Lattice parameters from a single-crystal on a fibre collected at beamline I19, EH2, using synchrotron radiation ( $\lambda=0.6889 \AA$ ) were determined to be $a=7.0194(2) \AA$ A. Data collected on a single-crystal of HMTD using a Bruker SMART APEXII diffractometer $\left(\mathrm{MoK}_{\alpha}\right)$ were in very good agreement with this initial dataset, giving $a=7.0195(1) \AA$ A. Measurements conducted on a single-crystal of HMTD on beamline I19, EH2 inside the sapphire capillary cell from before and after decompressing back to ambient pressure lay within error of each other, measuring $a=7.0190(3) \AA$ and $a=7.0187(3) \AA$ A respectively.

The resulting unit cell determinations collected under ambient pressure and temperature inside the cell for both HMTH and HMTD, therefore, match both experimental data collected here on other laboratory diffractometers, and values obtained from the literature. It would appear that accurate lattice parameters can, therefore, be determined from crystals inside the sapphire capillary cell, giving confidence that the instrument was well calibrated and the data collection strategy was appropriate. 


\subsubsection{The effect of pressure on HMTH and HMTD}

On increasing pressure, the $a$-axis for both HMTH and HMTD compressed by $0.34 \%$ and $0.39 \%$ between ambient pressure and 1000 bar respectively. This corresponded to extremely small changes in lattice parameters between each pressure point (100 bar steps), on average $0.003 \AA$ A. From 1000 bar, the pressure was decreased back to ambient pressure in 200 bar steps. For both HMTH and HMTD there was no indication of any hysteresis in the compression curves.

The compression curves of both HMTH and HMTD were fitted to a $2^{\text {nd }}$ order Birch-Murnaghan equation of state (EoS) to assess the compressibility using EoSFit ${ }^{32}$. For a $2^{\text {nd }}$ order Birch-Murnaghan the pressure, $p$, is given by:

$$
p=\frac{3 K_{0}}{2}\left[\left(\frac{V_{0}}{V}\right)^{7 / 3}-\left(\frac{V_{0}}{V}\right)^{5 / 3}\right]
$$

Where $K_{0}$ is the isothermal bulk modulus, $V_{0}$ is the volume at zero strain (ambient pressure) and $V$ the measured volume. In a $2^{\text {nd }}$ order Birch-Murnaghan the first pressure derivative $K_{0}^{\prime}=4$.

The bulk modulus of a material is a measure of its resistance to compression: the higher the bulk modulus, the harder the solid is to compress. Some representative values for scale: $6.6 \mathrm{GPa}$ for $\mathrm{Ru}_{3}(\mathrm{CO})_{12}, 13.1 \mathrm{GPa} \mathrm{L}$-alanine, $25 \mathrm{GPa} \mathrm{NaCl}, 37 \mathrm{GPa}$ for quartz and 440 GPa for diamond. ${ }^{33,34}$ The bulk modulus of HMTD was calculated to be significantly lower than that for HMTH ( $>3 \sigma$ difference) and is, therefore, a softer material.

Table 3.3 - Bulk modulus and ambient volume for both HMTH and HMTD.

\begin{tabular}{ccc} 
& $K_{0}$ & $V_{0}\left(a_{0}\right)$ \\
\hline HMTH & $9.45(18) \mathrm{GPa}$ & $346.938(42) \AA^{3}(7.0267 \AA)$ \\
HMTD & 8.31(9) GPa & $345.748(26) \AA^{3}(7.0187 \AA)$
\end{tabular}




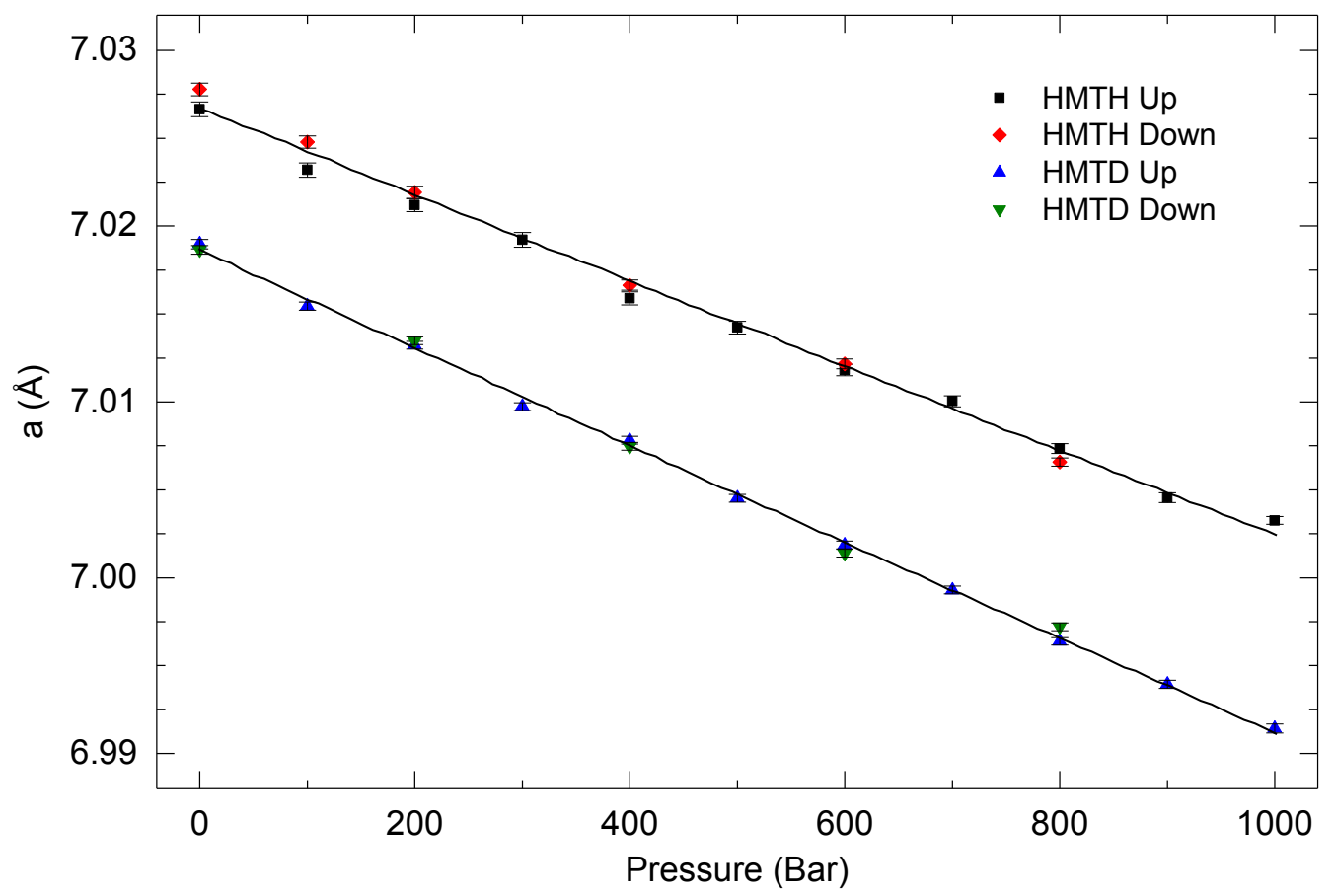

Figure 3.13 - Compression of the a-axis for both HMTH (black squares and red diamonds), and HMTD (blue and green triangles) along with their respective $2^{\text {nd }}$ order Birch-Murnaghan EoS fits. Data collected on increasing and decreasing pressure are indicated in the Figure key.

The H-D isotope effect (IE) has been shown to affect many different properties of materials at variable temperature and pressure. ${ }^{35}$ For example, altering phase change and Curie temperatures, ${ }^{36,37}$ inducing polymorphism, ${ }^{38}$ and can help to determine the reaction mechanism of certain chemical reactions (kinetic isotope effect, KIE). ${ }^{39}$

HMTD was found to have a slightly smaller unit cell volume than HMTH, $0.34 \%$. This follows the general rule for the H-D IE that lattice parameters decrease upon deuteration of a compound. ${ }^{40}$ This can be particularly strong for metal hydrides, $4.55 \%$ in the case of $\mathrm{H} / \mathrm{D}_{2}$ Se. However, the reverse can also be found, ${ }^{41}$ for example in short hydrogen-bonded systems, ${ }^{42-44}$ and for many compounds, almost no effect is noted. Therefore this IE is very system dependent.

The different compressibility observed here for HMTH/D is a somewhat more unusual IE. Examples have been noted where the IE changes the pressure of hydrogen-bond symmetrization in hydrogen bound systems at very high 
pressures, $100 \mathrm{kbar}$ for $\delta$-AlOOH ${ }^{45}$ and 600 kbar for ice X. ${ }^{46}$ This was not the case here as there are no formal hydrogen bonds present in HMTH/D, and the pressures here are much lower. Further work would be required to fully investigate the IE on the compressibility of HMTH/D and determine its origin, though we envisage that this would be hard to determine. The primary reason for this, is that very little structural changes occur within this pressure regime, which was unsurprising considering the highest pressures reached was 1000 bar. On comparing the ambient pressure and 1000 bar structures for both HMTH and HMTD for example, there is essentially no change to the cage-like structure and only very small changes in intermolecular distances, Figure 3.14. This can be quantified by comparing the root-mean-squared deviation (RMSD) for both structures when overlaid, which measure 0.0019 and 0.0015 for HMTH and HMTD respectively, as calculated in Mercury. ${ }^{47}$

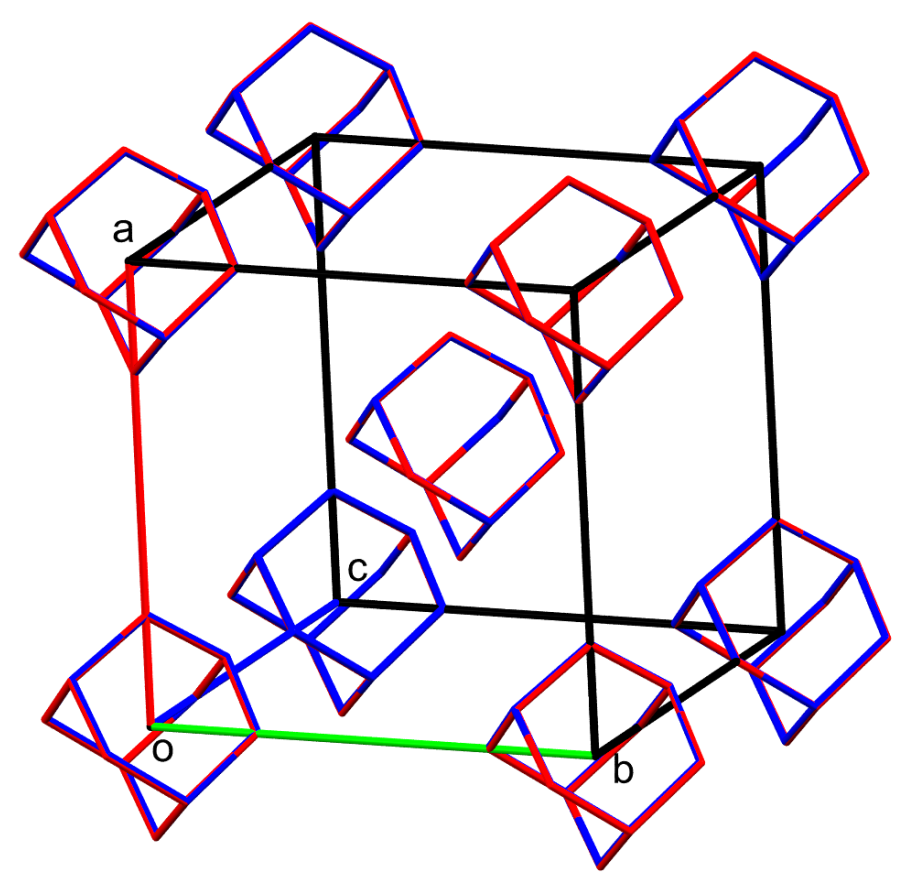

Figure 3.14 - An overlay of a packed unit cell of HMTH at ambient pressure, red, and at 1000 bar, blue. 
Chapter 3 - Sapphire Capillary Pressure Cell for In-Situ Single-Crystal Diffraction Measurements on Beamline I19 at Diamond Light Source

Table 3.4 - Unit cell parameters for HMTH

\begin{tabular}{ccc} 
Pressure (bar) & a-axis $(\mathbf{\AA})$ & Volume $\left(\AA^{3}\right)$ \\
\hline 1 & $7.0266(4)$ & $346.932(37)$ \\
100 & $7.0232(4)$ & $346.420(35)$ \\
200 & $7.0212(4)$ & $346.125(32)$ \\
300 & $7.0192(4)$ & $345.833(35)$ \\
400 & $7.0159(4)$ & $345.342(32)$ \\
500 & $7.0142(4)$ & $345.096(31)$ \\
600 & $7.0118(3)$ & $344.740(27)$ \\
700 & $7.0100(3)$ & $344.478(27)$ \\
800 & $7.0073(3)$ & $344.081(24)$ \\
900 & $7.0046(3)$ & $343.670(24)$ \\
1000 & $7.0033(2)$ & $343.479(19)$ \\
800 & $7.0066(2)$ & $343.969(20)$ \\
600 & $7.0122(3)$ & $344.792(23)$ \\
400 & $7.0166(3)$ & $345.452(25)$ \\
200 & $7.0219(3)$ & $346.233(29)$ \\
100 & $7.0248(3)$ & $346.657(30)$ \\
1 & $7.0278(4)$ & $347.099(30)$ \\
\hline
\end{tabular}

Table 3.5 - Unit cell parameters for HMTD

\begin{tabular}{ccc} 
Pressure (bar) & a-axis $(\mathbf{A})$ & Volume $\left(\mathbf{A}^{\mathbf{3}}\right)$ \\
\hline 1 & $7.0190(3)$ & $345.797(24)$ \\
100 & $7.0154(2)$ & $345.276(20)$ \\
200 & $7.0132(2)$ & $344.948(18)$ \\
300 & $7.0097(2)$ & $344.434(19)$ \\
400 & $7.0078(2)$ & $344.150(19)$ \\
500 & $7.0045(2)$ & $343.666(19)$ \\
600 & $7.0019(2)$ & $343.274(19)$ \\
700 & $6.9993(2)$ & $342.896(19)$ \\
800 & $6.9964(2)$ & $342.469(18)$ \\
900 & $6.9939(2)$ & $342.110(20)$ \\
1000 & $6.9914(3)$ & $341.741(21)$ \\
800 & $6.9972(2)$ & $342.591(19)$ \\
600 & $7.0014(2)$ & $343.205(18)$ \\
400 & $7.0075(2)$ & $344.099(18)$ \\
200 & $7.0135(2)$ & $344.984(19)$ \\
1 & $7.0187(3)$ & $345.749(21)$ \\
\hline
\end{tabular}




\subsection{Conclusions}

In conclusion, we have designed, manufactured and tested a new moderate pressure sapphire capillary pressure cell. This cell was optimised for use on the small molecule beamline I19 at the Diamond Light Source, where we have conducted tests and experiments regularly to pressures in excess of 1000 bar.

Here we have investigated the compression of the small organic molecule HMTH and its deuterated version HMTD. On loading both samples inside the capillary, we have demonstrated that very small changes in lattice parameters on both increasing and decreasing pressure can be determined precisely, between ambient pressure and 1000 bar. This has allowed the determination of accurate equations of state for both HMTH and HMTD. An isotope effect that increases the compressibility of HMTD over this pressure range and reduces the unit cell volume at ambient pressure in respect to HMTH. While the isotope effect on volume is well documented, anomalies in compressibility are less commonly observed. Precise equations of state measurements, like the ones presented here, offer the opportunity to develop new diffraction standards and pressure calibrants for high-pressure experiments.

HMTH and HMTD are two very simple systems; however, they demonstrate just the start of the potential uses for this cell. This cell reopens a regime of pressure long closed, during which time there have been great advances in the technology of single crystal X-ray diffraction and in the variety and complexity of species that scientists want to compress. As these species have become larger and more sophisticated in their chemical architecture, their compressibility has usually increased substantially rendering some not suitable for traditional high-pressure experiments using a DAC. This cell, in conjunction with the world-class instrumentation offered by Beamline I19, make it possible to investigate pressure responses for compounds that would otherwise have to be disregarded. 


\subsection{References}

1 Moggach, S. A., Allan, D. R., Parsons, S. \& Warren, J. E. Incorporation of a new design of backing seat and anvil in a Merrill-Bassett diamond anvil cell. Journal of Applied Crystallography 41, 249-251, doi:10.1107/S0021889808000514 (2008).

2 McKellar, S. C., Sotelo, J., Greenaway, A., Mowat, J. P. S., Kvam, O., Morrison, C. A., Wright, P. A. \& Moggach, S. A. Pore Shape Modification of a Microporous Metal-Organic Framework Using High Pressure: Accessing a New Phase with Oversized Guest Molecules. Chemistry of Materials 28, 466-473, doi:10.1021/acs.chemmater.5b02891 (2016).

3 Morosin, B. \& Schirber, J. E. X-ray investigation of a high-pressure phase of NH4F and ND4F. Journal of Chemical Physics 42, 1389-1390, doi:10.1063/1.1696126 (1965).

4 Morosin, B. \& Schirber, J. E. Low-temperature, high-pressure X-ray cell. Journal of Applied Crystallography 7, 295-296, doi:10.1107/S0021889874009575 (1974).

5 Morosin, B. \& Schirber, J. E. Changes in atomic positions for Sb and Bi with hydrostatic pressure. Physics Letters A A 30, 512-513, doi:10.1016/03759601(69)90277-1 (1969).

6 Kundrot, C. E. \& Richards, F. M. Collection and processing of X-ray diffraction data from protein crystals at high pressure. Journal of Applied Crystallography 19, 208-213, doi:10.1107/S0021889886089562 (1986).

7 Urayama, P., Phillips Jr, G. N. \& Gruner, S. M. Probing Substates in Sperm Whale Myoglobin Using High-Pressure Crystallography. Structure 10, 5160, doi:http://dx.doi.org/10.1016/S0969-2126(01)00699-2 (2002).

8 Collins, M. D., Hummer, G., Quillin, M. L., Matthews, B. W. \& Gruner, S. M. Cooperative water filling of a nonpolar protein cavity observed by highpressure crystallography and simulation. Proceedings of the National Academy of Sciences of the United States of America 102, 16668-16671, doi:10.1073/pnas.0508224102 (2005).

9 Collins, M. D., Quillin, M. L., Hummer, G., Matthews, B. W. \& Gruner, S. M. Structural Rigidity of a Large Cavity-containing Protein Revealed by 
High-pressure Crystallography. Journal of Molecular Biology 367, 752-763, doi:http://dx.doi.org/10.1016/j.jmb.2006.12.021 (2007).

10 Suzuki, Y., Tsukamoto, M., Sakuraba, H., Matsumoto, M., Nagasawa, M. \& Tamura, K. Design of a standalone-type beryllium vessel for high-pressure protein crystallography. Review of Scientific Instruments 81, 084302, doi:http://dx.doi.org/10.1063/1.3478000 (2010).

11 Hamaya, N., Kuroiwa, Y. \& Fujii, Y. A unique high pressure apparatus for X-ray diffraction studies of phase transitions up to 5 kbar. Nuclear Instruments and Methods in Physics Research Section B: Beam Interactions with Materials and Atoms 29, 537-543, doi:http://dx.doi.org/10.1016/0168583X(87)90066-8 (1987).

12 Probert, M. R., Robertson, C. M., Coome, J. A., Howard, J. A. K., Michell, B. C. \& Goeta, A. E. The XIPHOS diffraction facility for extreme sample conditions. Journal of Applied Crystallography 43, 1415-1418, doi:10.1107/S0021889810041282 (2010).

13 Yufit, D. S. \& Howard, J. A. K. Simple pressure cell for single-crystal X-ray crystallography. Journal of Applied Crystallography 38, 583-586, doi:10.1107/s0021889805011258 (2005).

14 Paradis, N., Le Gac, F., Guionneau, P., Largeteau, A., Yufit, D., Rosa, P., Létard, J.-F. \& Chastanet, G. Effects of Internal and External Pressure on the [Fe(PM-PEA)2(NCS)2] Spin-Crossover Compound (with PM-PEA = N(2'-pyridylmethylene)-4-(phenylethynyl)aniline). Magnetochemistry 2, 15 (2016).

15 Dickinson, R. G. \& Raymond, A. L. The crystal structure of hexamethylenetetramine. Journal of the American Chemical Society 45, 22-29, doi:10.1021/ja01654a003 (1923).

16 Gonell, H. W. \& Mark, H. Röntgenographische Bestimmung der Strukturformel des Hexamethylentetramins. Zeitschrift fur Physikalische Chemie 107U, 181, doi:10.1515/zpch-1923-10715 (1923).

17 Mitchinson, A. in Nature Milestones in Crystallography (Nature, 2014).

18 Duckworth, J. A. K., Willis, B. T. M. \& Pawley, G. S. Neutron diffraction study of the atomic and molecular motion in hexamethylenetetramine. 
Chapter 3 - Sapphire Capillary Pressure Cell for In-Situ Single-Crystal Diffraction

Measurements on Beamline I19 at Diamond Light Source

Acta Crystallographica Section A 26, 263-271, doi:10.1107/S0567739470000645 (1970).

19 Terpstra, M., Craven, B. M. \& Stewart, R. F. Hexamethylenetetramine at 298 K: new refinements. Acta Crystallographica Section A 49, 685-692, doi:10.1107/S0108767393001023 (1993).

20 Mencke, A., Cheng, A. c. \& Caffrey, M. A simple apparatus for time-resolved $\mathrm{x}$-ray diffraction biostructure studies using static and oscillating pressures and pressure jumps. Review of Scientific Instruments 64, 383-389, doi:10.1063/1.1144261 (1993).

21 Brooks, N. J. Personal Comunication. (2016).

22 Black, D. R., Windover, D., Henins, A., Filliben, J. \& Cline, J. P. Certification of Standard Reference Material 660B. Powder Diffraction 26, 155-158, doi:10.1154/1.3591064 (2012).

23 CrysAlis PRO (Agilent, Agilent Technologies Ltd, Yarnton, Oxfordshire, England., 2014).

24 APEX3 v. v2016.9-0 (Bruker AXS Inc., Madison, Wisconsin, USA, 2016).

25 Johnson, N. T. \& Probert, M. R. cbf_to_sfrm 2016 (alpha version), <https://github.com/nu-xtal-tools/cbf_to_sfrm> (2016).

26 Winter, G. xia2: an expert system for macromolecular crystallography data reduction. Journal of Applied Crystallography 43, 186-190, doi:10.1107/S0021889809045701 (2010).

27 Cosier, J. \& Glazer, A. M. A nitrogen-gas-stream cryostat for general X-ray diffraction studies. Journal of Applied Crystallography 19, 105-107, doi:10.1107/S0021889886089835 (1986).

28 SAINT v. 8.34A (Bruker AXS Inc., Madison, Wisconsin, USA, 2014).

29 SADABS v. 2008-1 ed. (Bruker AXS Inc., Madison, Wisconsin, USA, 2014/15).

30 Betteridge, P. W., Carruthers, J. R., Cooper, R. I., Prout, K. \& Watkin, D. J. CRYSTALS version 12: software for guided crystal structure analysis. Journal of Applied Crystallography 36, 1487-1487, doi:10.1107/s0021889803021800 (2003). 
31 Stevens, E. D. \& Hope, H. Accurate positional and thermal parameters of hexamethylenetetramine from K-shell X-ray-diffraction data. Acta Crystallographica Section A 31, 494-498, doi:10.1107/s0567739475001064 (1975).

32 Gonzalez-Platas, J., Alvaro, M., Nestola, F. \& Angel, R. EosFit7-GUI: a new graphical user interface for equation of state calculations, analyses and teaching. Journal of Applied Crystallography 49, 1377-1382, doi:10.1107/S1600576716008050 (2016).

33 Funnell, N. P., Marshall, W. G. \& Parsons, S. Alanine at 13.6 GPa and its pressure-induced amorphisation at 15 GPa. Crystengcomm 13, 5841-5848, doi:10.1039/ClCE05487B (2011).

34 Slebodnick, C., Zhao, J., Angel, R., Hanson, B. E., Song, Y., Liu, Z. \& Hemley, R. J. High Pressure Study of Ru3(CO)12 by X-ray Diffraction, Raman, and Infrared Spectroscopy. Inorganic Chemistry 43, 5245-5252, doi:10.1021/ic049617y (2004).

35 Van Hook, W. A. Isotope effects in chemistry. Nukleonika 56, 217-240 (2011).

36 Stritzker, B. \& Buckel, W. Superconductivity in the palladium-hydrogen and the palladium-deuterium systems. Zeitschrift für Physik A Hadrons and nuclei 257, 1-8, doi:10.1007/bf01398191 (1972).

37 McMahon, M. I., Nelmes, R. J., Kuhst, W. F., Dorwarth, R., Piltz, R. O. \& Tun, Z. Geometric effects of deuteration on hydrogen-ordering phase transitions. Nature 348, 317-319 (1990).

38 Ip, B. C. K., Shenderovich, I. G., Tolstoy, P. M., Frydel, J., Denisov, G. S., Buntkowsky, G. \& Limbach, H.-H. NMR Studies of Solid Pentachlorophenol-4-Methylpyridine Complexes Exhibiting Strong OHN Hydrogen Bonds: Geometric H/D Isotope Effects and Hydrogen Bond Coupling Cause Isotopic Polymorphism. The Journal of Physical Chemistry A 116, 11370-11387, doi:10.1021/jp305863n (2012).

39 Melander, L. C. S. \& Saunders, W. H. Reaction rates of isotopic molecules. (Wiley, 1980). 
40 Kogan, V. S. Isotope effects in the structural properties of solids. Soviet Physics Uspekhi 5, 951-975 (1963).

41 David, A. K., Martin, T. D., John, S. O. E., Andrew, L. G., Lars, P. \& Matthew, G. T. The hydrogen-bonding transition and isotope-dependent negative thermal expansion in $\mathrm{H} 3 \mathrm{Co}(\mathrm{CN})$ 6. Journal of Physics: Condensed Matter 22, $404202(2010)$.

42 Nordman, C. E. \& Lipscomb, W. N. Note on the Hydrogen-Deuterium Isotope Effect in Crystals. The Journal of Chemical Physics 19, 1422-1422, doi:10.1063/1.1748075 (1951).

43 Nordman, C. E. \& Lipscomb, W. N. Isotope Effect and Thermal Expansion Coefficients of Crystals. The Journal of Chemical Physics 21, 2077-2077, doi:10.1063/1.1698754 (1953).

44 Ubbelohde, A. R. \& Gallagher, K. J. Acid-base effects in hydrogen bonds in crystals. Acta Crystallographica 8, 71-83, doi:10.1107/S0365110X55000340 (1955).

45 Sano-Furukawa, A., Kagi, H., Nagai, T., Nakano, S., Fukura, S., Ushijima, D., Iizuka, R., Ohtani, E. \& Yagi, T. Change in compressibility of $\delta$-AlOOH and $\delta$-AlOOD at high pressure: A study of isotope effect and hydrogenbond symmetrization. American Mineralogist 94, 1255-1261, doi:10.2138/am.2009.3109 (2009).

46 Goncharov, A. F., Struzhkin, V. V., Somayazulu, M. S., Hemley, R. J. \& Mao, H. K. Compression of Ice to 210 Gigapascals: Infrared Evidence for a Symmetric Hydrogen-Bonded Phase. Science 273, 218-220, doi:10.1126/science.273.5272.218 (1996).

47 Macrae, C. F., Bruno, I. J., Chisholm, J. A., Edgington, P. R., McCabe, P., Pidcock, E., Rodriguez-Monge, L., Taylor, R., van de Streek, J. \& Wood, P. A. Mercury CSD 2.0 - new features for the visualization and investigation of crystal structures. Journal of Applied Crystallography 41, 466-470, doi:10.1107/S0021889807067908 (2008). 
- Chapter 4 -

\section{The Effect of Pressure on $\mathrm{Sc}_{2} \mathrm{BDC}_{3}$ using the Sapphire Capillary Pressure Cell}




\subsection{Introduction}

Scandium 1,4-benzenedicarboxylate, $\mathrm{Sc}_{2} \mathrm{BDC}_{3}$, is a very stable small pore metalorganic framework (MOF). ${ }^{1}$ The structure is formed from $\mathrm{ScO}_{6}$ octahedral nodes, where all oxygen atoms are part of the linking $\mathrm{BDC}$ ligands. $\mathrm{Sc}_{2} \mathrm{BDC}_{3}$ has onedimensional channels with a cross-section of $\approx 4 \AA$ in diameter that have been shown to be accessible to small molecules such as $\mathrm{CO}_{2}$ (kinetic diameter $\approx 3 \AA$ ) and small alkanes (methane, ethane, propane; $\approx 4 \AA$ ). ${ }^{2}$

Under ambient temperature and pressure conditions, $\mathrm{Sc}_{2} \mathrm{BDC}_{3}$ crystallizes in the orthorhombic space group Fddd, having two independent BDC linker molecules (Groups 1 and 2; Figure 4.1a), the phenyl rings of which are parallel to the $a$-axis (i.e., parallel to the principal framework channel direction).

Longer unbranched and branched alkanes (from $C_{5}$ to $C_{8}$ ) have been found to enter the structure despite being oversized for the channel. ${ }^{3}$ This was achieved by using high-pressure to overcome the steric hindrance. Linear hydrocarbons (such as $n$ pentane) were found to be taken up most readily; while tertiary dibranched hydrocarbons, such as 2,2-dimethylbutane, were not absorbed at all and led to direct compression, and amorphisation of the sample.

As these molecules entered the $\mathrm{Sc}_{2} \mathrm{BDC}_{3}$ it was found that the framework structure altered in response to these oversized guests through rotation of the phenyl ring of the Group-2 BDC linker. The uptake of either linear or dibranched

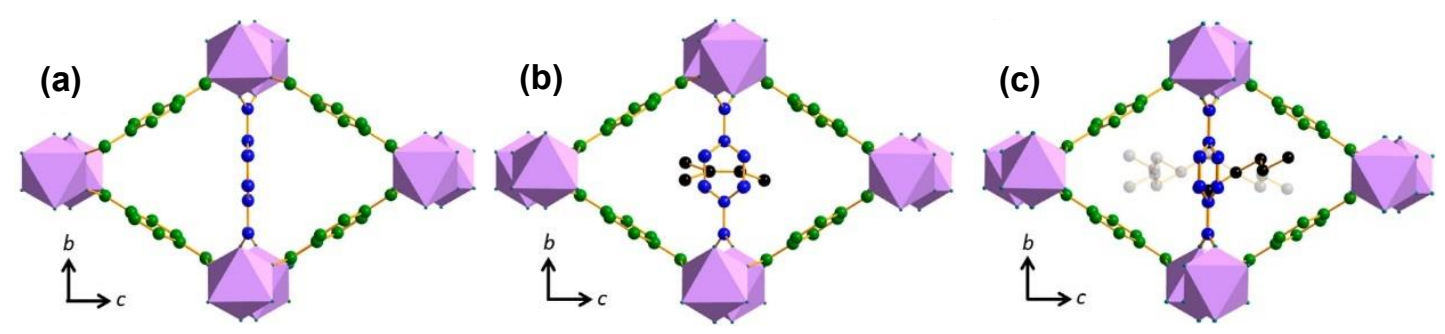

Figure 4.1 - Structure of (a) orthorhombic $\mathrm{S}_{2} \mathrm{BDC}_{3}$ viewed parallel to the crystallographic a-axis, with $\mathrm{ScO}_{6}$ octahedra coloured purple and $\mathrm{BDC}$ linkers coloured by symmetry equivalence. $\mathrm{H}$ atoms are omitted for clarity. In (a), Groups 1 and 2 are coloured green and blue, respectively. In (b) and (c), guest molecules are shown in black. For clarity, an ordered isopentane molecule is shown in (b). The npentane positions disordered by symmetry are shown in gray in (c). Figure adapted from McKellar et al. (2016). 
hydrocarbons into the pores resulted in the phenyl ring rotating into two statically disordered (50:50 occupancy) positions (Figure 4.2b).

This effect was found to be most significant on uptake of isopentane (2methylbutane) henceforth referred to as $\mathrm{Sc}_{2} \mathrm{BDC}_{3}(\mathrm{ip})$; Figure $4.1 \mathrm{~b}$ and Figure $4.2 \mathrm{~b} \& \mathrm{~d}$ represent the structure of $\mathrm{Sc}_{2} \mathrm{BDC}_{3}(\mathrm{ip})$ at $4 \mathrm{kbar}(0.4 \mathrm{GPa})$. At this pressure, and on uptake of isopentane the Group- 2 phenyl rings rotated by $52.4^{\circ}$ (and $-52.4^{\circ}$ by symmetry) with respect to the (001) plane (see Table 4.1 ). By this pressure, the pore size and shape had changed dramatically, from adjacent triangular channels to rhomboidal cavities (Figure 4.2c\&d). This was the first and only pressure point $\mathrm{Sc}_{2} \mathrm{BDC}_{3}(\mathrm{ip})$ collected due to amorphisation above this pressure.

Ambient

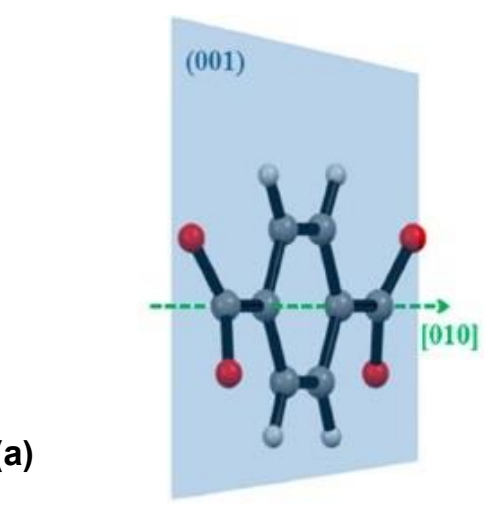

4 kbar

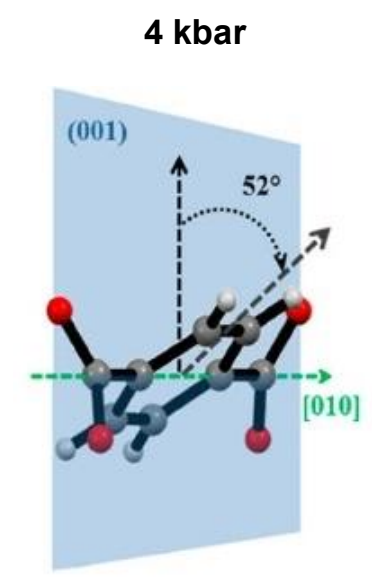

(a)
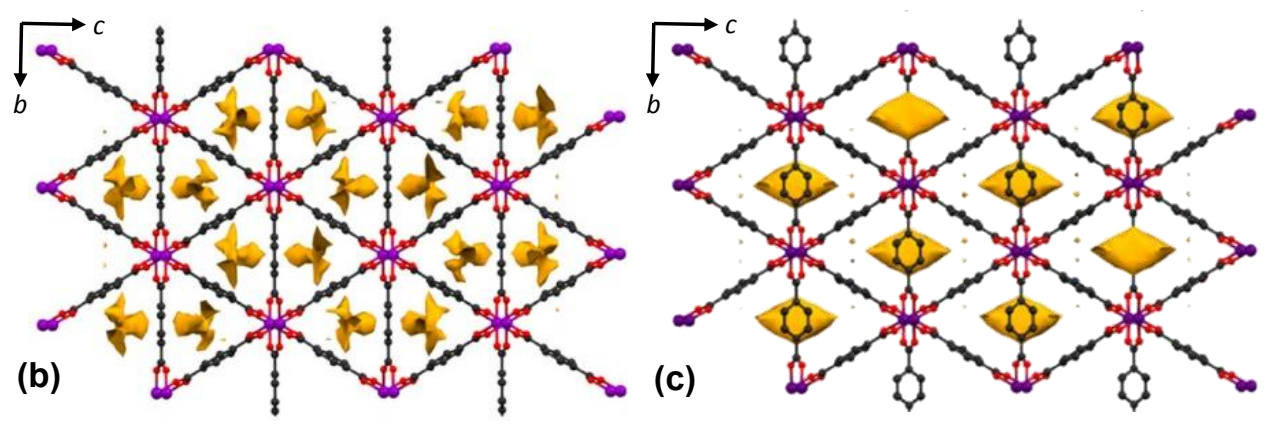

Figure 4.2 -Scheme of the ring rotation around [010] of Group-2 phenyl rings with respect to the (001) plane. (a) The native $\mathrm{Sc}_{2} \mathrm{BDC}_{3}$ structure; (b) the high-pressure guest-included structure $\mathrm{Sc}_{2} \mathrm{BDC}_{3}$ (ip). Only one of the statistically disordered rings is shown for clarity. (c) Solvent accessible surface (yellow) in one unit cell of $\mathrm{Sc}_{2} \mathrm{BDC}_{3}$. (d) Solvent accessible surface (yellow) in one unit cell of $\mathrm{Sc}_{2} \mathrm{BDC}_{3}$ (ip). Isopentane molecules are not shown in (d) to allow clear representation of the modified pore space. Figure adapted from McKellar et al. (2016). 
Table 4.1 - Structures of isopentane and $n$-pentane with their kinetic diameters along with a summary of the rotation angle of the Group-2 BDC phenyl rings for previous high-pressure and in-situ immersion experiments on $\mathrm{Sc}_{2} \mathrm{BDC}_{3}$. Channel content calculated using SQUEEZE is also included. ${ }^{3}$

\begin{tabular}{|c|c|c|c|c|c|}
\hline Solvent & $\begin{array}{c}\text { Molecular } \\
\text { structure }\end{array}$ & $\begin{array}{c}\text { Kinetic } \\
\text { diameter }(\AA)\end{array}$ & $\begin{array}{c}\text { Pressure } \\
\text { (kbar) }\end{array}$ & $\begin{array}{l}\text { Rotation } \\
\text { angle }\left({ }^{\circ}\right)\end{array}$ & $\begin{array}{l}\text { No. of molecules } \\
\text { per channel }\end{array}$ \\
\hline \multirow{2}{*}{ Isopentane } & & \multirow{2}{*}{4.5} & Ambient & 0 & - \\
\hline & & & 4 & 52.4 & 0.87 \\
\hline \multirow{4}{*}{$n$-pentane } & & \multirow{4}{*}{5.0} & Ambient & 14.0 & - \\
\hline & & & 2 & 20.6 & 0.47 \\
\hline & & & 5 & 43.7 & 1.09 \\
\hline & & & 8 & 35.0 & 1.10 \\
\hline
\end{tabular}

Upon immersion under ambient pressure conditions in $n$-pentane (hereafter reffered to as $\left.\mathrm{Sc}_{2} \mathrm{BDC}_{3}(\mathrm{np})\right)$, the adsorbed linear hydrocarbon caused a rotation of the Group-2 phenyl ring by $14^{\circ}$, suggesting that $\mathrm{Sc}_{2} \mathrm{BDC}_{3}$ easily adsorbs linear hydrocarbons, Table 4.1. In a similar immersion experiment, no crystallographic evidence for uptake (or rotation of the Group-2 phenyl ring) caused by the branched and bulkier isopentane was observed under ambient conditions.

High-pressure experiments in $n$-pentane showed an increase in rotation of the Group-2 phenyl ring to $20.8^{\circ}$ at 2 kbar, increasing to $43.7^{\circ}$ by 5 kbar. This was less than in $\mathrm{Sc}_{2} \mathrm{BDC}_{3}(\mathrm{ip})$; however, the uptake of $n$-pentane stabilised the framework, and allowed it to maintain crystallinity up to $8 \mathrm{kbar}(0.8 \mathrm{GPa})$, Table 4.1 . On increasing pressure above $4 \mathrm{kbar}$ in isopentane, $\mathrm{Sc}_{2} \mathrm{BDC}_{3}$ underwent a reversible crystalline-amorphous phase transition. The structure and the shape of the guest molecules, and their location within the cavity, therefore resulted in distinct differences in the structural behaviour of $\mathrm{Sc}_{2} \mathrm{BDC}_{3}$.

$\mathrm{Sc}_{2} \mathrm{BDC}_{3}$ undergoes similar structural deformations on uptake of both isopentane and $n$-pentane, but with subtle differences. The uptake of $n$-pentane was observed at ambient pressure, and stabilized compression of the crystalline phase to much higher pressures. On compression using isopentane, no uptake was observed under ambient pressure, however on loading the DAC at $4 \mathrm{kbar}$, a large rotation of the Group-2 phenyl ring took place. Unfortunately, due to limitations in control of pressure increments when using a $\mathrm{DAC}$, what happens to $\mathrm{Sc}_{2} \mathrm{BDC}_{3}$ (ip) below 
Chapter 4 - The Effect of Pressure on $\mathrm{Sc}_{2} \mathrm{BDC}_{3}$ Using the Sapphire Capillary Pressure Cell

4 kbar is currently unknown. Without detailed structural analysis capturing the intermediate behaviour as the uptake takes place, these subtle differences are difficult to understand fully. Here, we have investigated the uptake of both $n$ pentane and isopentane into $\mathrm{Sc}_{2} \mathrm{BDC}_{3}$ using the sapphire capillary cell. 


\subsection{Experimental}

Note: The synthesis of materials was carried out by Dr John Mowat, Berenice Gonzalez and Dr Alex Greenaway from the University of St. Andrews. ${ }^{2}$

\subsubsection{Synthesis of $\mathrm{Sc}_{2} \mathrm{BDC}_{3}$ via a solvothermal route}

$\mathrm{Sc}\left(\mathrm{NO}_{3}\right)_{3} 4 \mathrm{H}_{2} \mathrm{O}$ (Alfa Aesar, $0.34 \mathrm{~g}, 99.9 \%$, $1.1 \mathrm{mmol}$ ), 1,4-benzenedicarboxylate (Aldrich, $0.170 \mathrm{~g}, 99 \%, 1 \mathrm{mmol}$ ), $\mathrm{H}_{2} \mathrm{O}_{2}$ (Aldrich, 30 wt \% in water, $0.25 \mathrm{~mL}$ ), pyridine (Fisher, $0.5 \mathrm{~mL}, 99 \%$ ), and dimethylformamide (DMF; Aldrich, $10 \mathrm{~mL}$, 99.8\%) were sealed in a Parr autoclave with a $23 \mathrm{~mL}$ Teflon liner. The mixture was subsequently heated to $493 \mathrm{~K}$ and allowed to react for two days. The autoclave was cooled, and the final product was filtered and washed with DMF. Large single crystals $(\sim 150 \mu \mathrm{m})$ were obtained, Figure 4.3 .

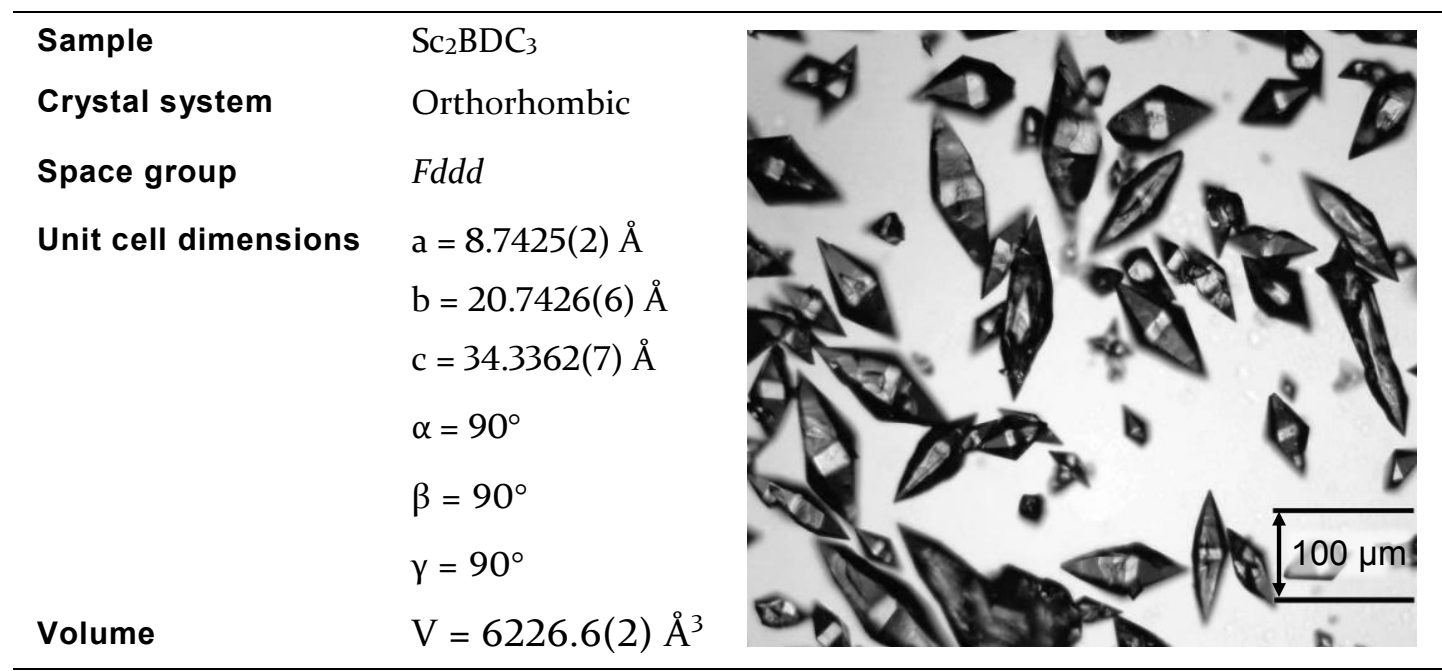

Figure 4.3 - Crystallographic information for $\mathrm{Sc}_{2} \mathrm{BDC}_{3}$ at ambient pressure and temperature. Image from Miller et al., 2009. ${ }^{2}$

\subsubsection{Moderate pressure crystallographic studies}

Two compression series were performed on $\mathrm{Sc}_{2} \mathrm{BDC}_{3}$ using the sapphire capillary pressure cell to investigate whether any structural deformation or uptake of both $n$-pentane and isopentane could be observed. Diffraction data were collected on beamline I19, Experimental Hutch 2 (EH2) at the Diamond Light Source, Rutherford Appleton Laboratory. ${ }^{4}$ EH2 is equipped with a Newport 4-circle goniometer with a DECTRIS PILATUS 300K hybrid-pixel detector using 
synchrotron radiation $(\lambda=0.6889 \AA)$. Data collection was carried out using an exposure time and a step size of 0.2 second and 0.2 degrees respectively using the strategy outlined previously in Chapter 3, §3.4.3.3. CrysAlisPro (version: 171.38.46) was used for integration, absorption correction and space group determination. ${ }^{5}$

For compression in isopentane, a single crystal of $\mathrm{Sc}_{2} \mathrm{BDC}_{3}$ was mounted onto a carbon fibre and collected at ambient pressure. This was then placed into a $1 \mathrm{~mm}$ OD x $0.6 \mathrm{~mm}$ ID sapphire cell and the pressure was increased from 100 bar up to a maximum pressure of 1200 bar in 100 bar steps.

During data reduction, a resolution cut-off of $0.8 \AA ̊$ was used for all datasets. All structures were refined against $\left|F^{2}\right|$ using the program CRYSTALS. ${ }^{6}$ Up to 1100 bar all non-hydrogen atoms were freely refined anisotropically. Hydrogen atoms were geometrically placed and constrained to ride there bonded atoms. For the final pressure point at 1200 bar, it was necessary to model the phenyl ring of the Group-2 BDC linker as a 50:50 split over two positions. For this model, 1-2 and 1-3 restraints, as well as vibrational and thermal similarity restraints were applied to the disordered Group-2 BDC linker. Analysis of the solvent accessible volume and the residual electron density were performed using the SQUEEZE algorithm within PLATON with a probe radius of $1.2 \AA .{ }^{7}$ The results from the residual electron density calculations were used to determine the uptake of solvent at each pressure point.

For compression in $n$-pentane, a single crystal of $\mathrm{Sc}_{2} \mathrm{BDC}_{3}$ was similarly loaded into a larger $1.4 \mathrm{~mm}$ OD x $1 \mathrm{~mm}$ ID sapphire cell. Data were collected from 50 bar up to a maximum pressure of 900 bar.

A resolution cut off of $0.7 \AA$ was used for all data sets and refinements were carried out using CRYSTALS against $\left|F^{2}\right|{ }^{6}$ For the first two pressure points at 50 and 200 bar, all non-hydrogen atoms were freely refined anisotropically. Hydrogen atoms were geometrically placed and constrained to ride there bonded atoms. By 400 bar the phenyl ring of the Group-2 BDC linker became disordered and so was 
modelled over two positions (50:50) by symmetry. $1-2$ and 1-3 restraints, as well as vibrational and thermal similarity restraints were applied to the Group-2 BDC linker.

The final pressure point at 900 bar the phenyl ring of the Group-2 BDC linker was split into a 25:25:25:25 four-way disordered split. At this pressure 1-2 and 1-3 restraints, as well as vibrational and thermal similarity restraints were applied to all non-hydrogen atoms (except for Sc). The Group-2 BDC ligands were also restrained to be planar.

The solvent accessible volume and residual electron density were again analysed using SQUEEZE with the probe radius of $1.2 \AA .{ }^{7}$ This was used to determine the uptake of solvent at each pressure point. 


\subsection{Results and Discussion}

\subsubsection{Unit cell compression}

The different behaviour of $\mathrm{Sc}_{2} \mathrm{BDC}_{3}$ under compression in isopentane $\left(\mathrm{Sc}_{2} \mathrm{BDC}_{3}(\mathrm{ip})\right)$ and $n$-pentane $\left(\mathrm{Sc}_{2} \mathrm{BDC}_{3}(\mathrm{np})\right)$ can be seen clearly in the unit cell compression. For $\mathrm{Sc}_{2} \mathrm{BDC}_{3}$ (ip) the unit cell volume steadily decreased with increasing pressure (Figure 4.4 , blue diamonds). This is contrasted by the behaviour in $\mathrm{Sc}_{2} \mathrm{BDC}_{3}(\mathrm{np})$ (Figure 4.4, black squares) where the unit cell volume actually increased as a function of pressure, by $7.6 \AA^{3}(0.12 \%)$, to 900 bar. This behaviour is very similar to that previously seen on uptake of methanol at highpressure $(0.7 \%$ to 6 kbar $){ }^{8}$
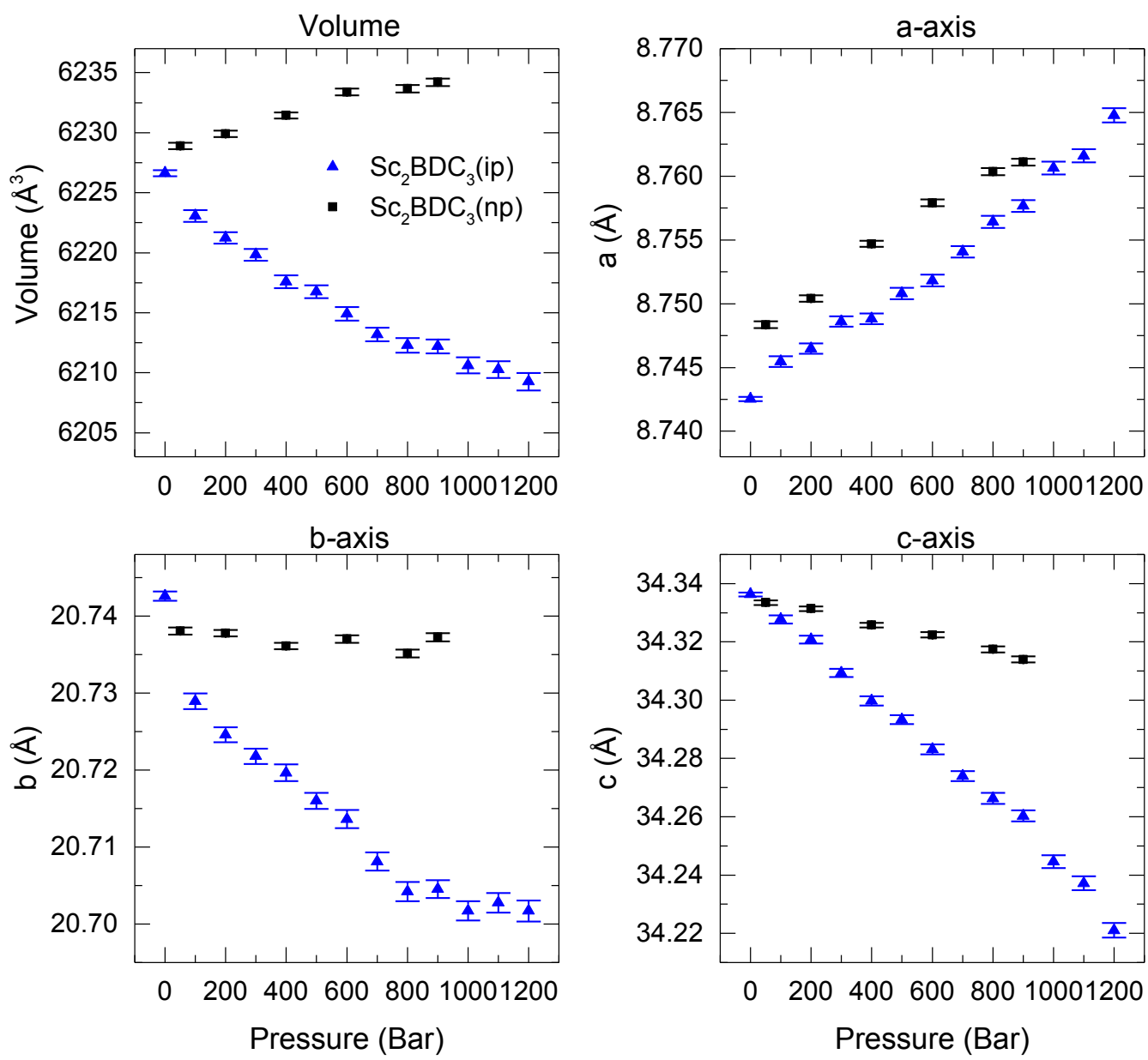

Figure 4.4 - Unit cell volume and lattice parameters as a function of pressure for $\mathrm{Sc}_{2} \mathrm{BDC}_{3}(\mathrm{ip})$, blue triangles, and $\mathrm{Sc}_{2} \mathrm{BDC}_{3}(\mathrm{np})$, black squares. 
The differences seen in the unit cell volume compression is driven by the different responses along the unit cell axes. There is a very similar elongation along the $a$-axis for both $\mathrm{Sc}_{2} \mathrm{BDC}_{3}(\mathrm{ip})$ and $\mathrm{Sc}_{2} \mathrm{BDC}_{3}(\mathrm{np})$. For $\mathrm{Sc}_{2} \mathrm{BDC}_{3}(\mathrm{ip})$ the $b$ and $c$-axes are compressed steadily with increasing pressure while for $\mathrm{Sc}_{2} \mathrm{BDC}_{3}(\mathrm{np})$ there is far less compression of the $b$ and $c$-axes. The $b$ and $c$-axes describe the pore channel diameter as they lie parallel to the face of the porous channels, Figure 4.1.

\subsubsection{The effect on $\mathrm{Sc}_{2} \mathrm{BDC}_{3}$ on uptake of isopentane}

The structures of $\mathrm{Sc}_{2} \mathrm{BDC}_{3}(\mathrm{ip})$ were closely monitored as a function of pressure, in particular, the motion of the phenyl ring of the Group-2 BDC linker was monitored. The Group-2 BDC linker has been previously shown to rotate by $52.4^{\circ}$ along [010] into two statically disordered (50:50 occupancy) positions upon the application of 4 kbar of pressure, Figure $4.2 \mathrm{~b} \& \mathrm{~d} .{ }^{3}$ In order to track any rotation of the phenyl ring the principal axes of the atomic displacement parameters (ADPs) for the carbon atom, C7, and its symmetry equivalents that make up the 2,3,5\&6-positions of the phenyl ring were monitored as a function of pressure, Figure 4.5 .

As the pressure was increased, the ellipsoid for C7 became progressively more prolate. By 1200 bar the magnitude of the principal ADP of the un-split model measured $0.26 \AA^{2}$. At 1200 bar, it was therefore more reasonable to model C7 as a

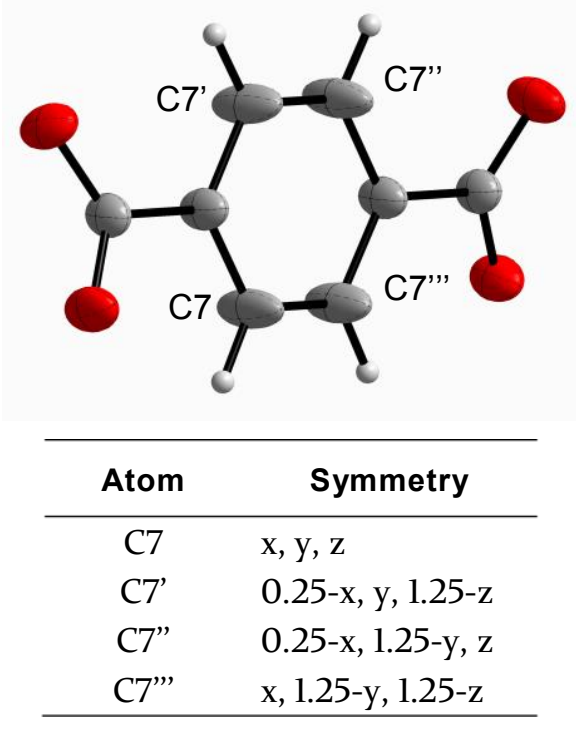

Figure 4.5 - Group-2 BDC linker with C7 and its symmetry equivalents. 
50:50 split disordered BDC ligand, Figure 4.6. The disorder model was stable during refinement and reduced the largest principal axis of the ADP to $0.18 \AA^{2}$. The atoms in the split model were, however, still prolate, suggesting dynamic rather than static disorder. With the split model, the angle of rotation for the phenyl ring along [010] with respect to the (001) plane was calculated to be $14.9^{\circ}$, Figure 4.6. This rotation is much less than the $52.4^{\circ}$ seen at high-pressure ( $4 \mathrm{kbar}$ ) therefore this rotation must continue to slowly increase with pressure.

It was not possible to model the disordered solvent in the channels with an atomistic model. To determine the extent of uptake of solvent with pressure the SQUEEZE algorithm was therefore used to calculate the electron density in the pores. At ambient pressure, before exposure to solvent, the channels of $\mathrm{Sc}_{2} \mathrm{BDC}_{3}$ were essentially empty (18 $\mathrm{e}^{-}$for a void volume of $\left.2186 \AA^{3}\right)$. For $\mathrm{Sc}_{2} \mathrm{BDC}_{3}(\mathrm{ip})$ there was an immediate uptake of isopentane into the channels on loading at 100 bar, Figure 4.7. This absorption appeared to level off at approximately 0.8 molecules per channel by 700 bar. The SQUEEZE output, along with an increase in the principal axis of the C7-phenyl carbon atom ADP, and the need for a 'split' disorder model at 1200 bar, demonstrates that uptake of isopentane into the channels with

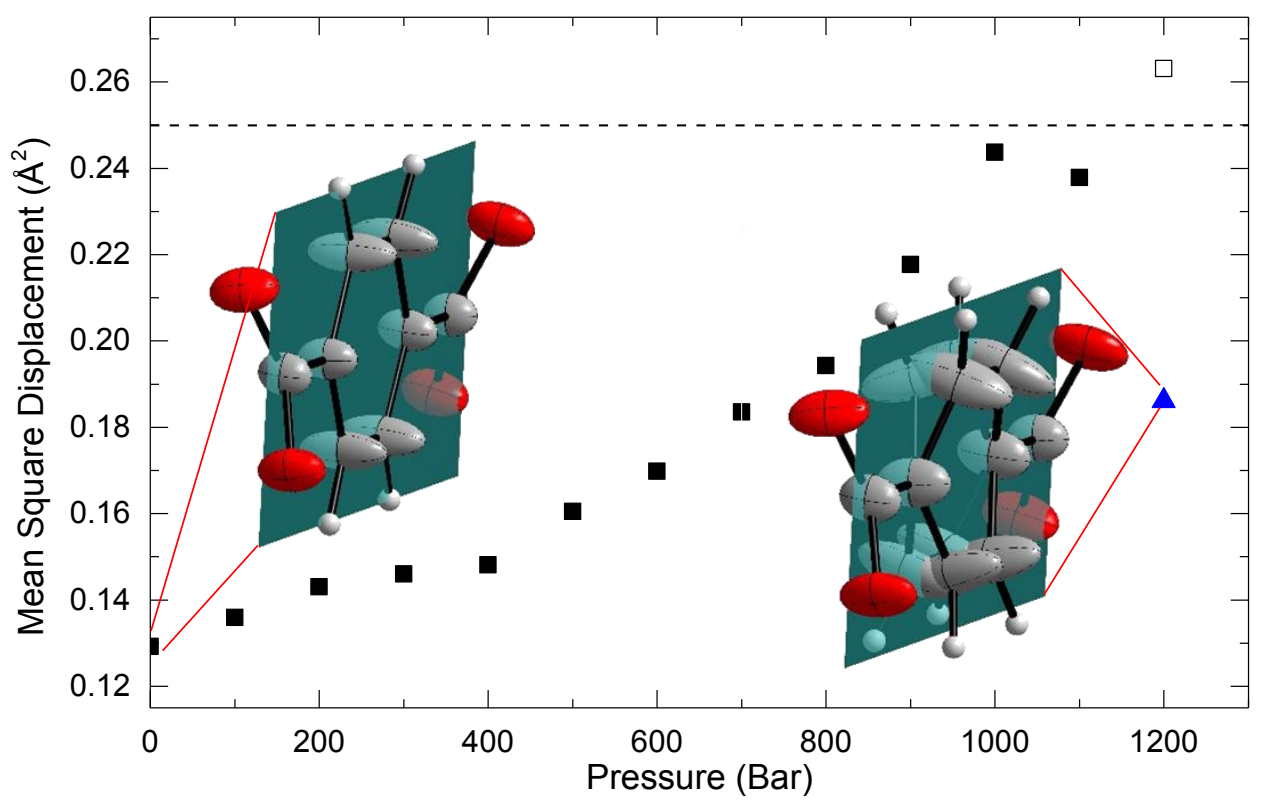

Figure 4.6 - The magnitude of the principle ADP of C7 as a function of pressure for $\mathrm{Sc}_{2} \mathrm{BDC}_{3}$ (ip) as an un-split model, black squares, and a 50:50 split model, blue triangle. Open square at 1200 bar above $0.25 \AA^{2}$ therefore the 50:50 split model is employed. Inserts of the Group-2 $\mathrm{BDC}$ linker of; $\mathrm{Sc}_{2} \mathrm{BDC}_{3}$ at ambient pressure, left; and $\mathrm{Sc}_{2} \mathrm{BDC}_{3}$ (ip) as a 50:50 split model at 1200 bar, right. Both inserts show nonhydrogen atoms with $50 \%$ probability ellipsoids and the (001) plane shown in green. 


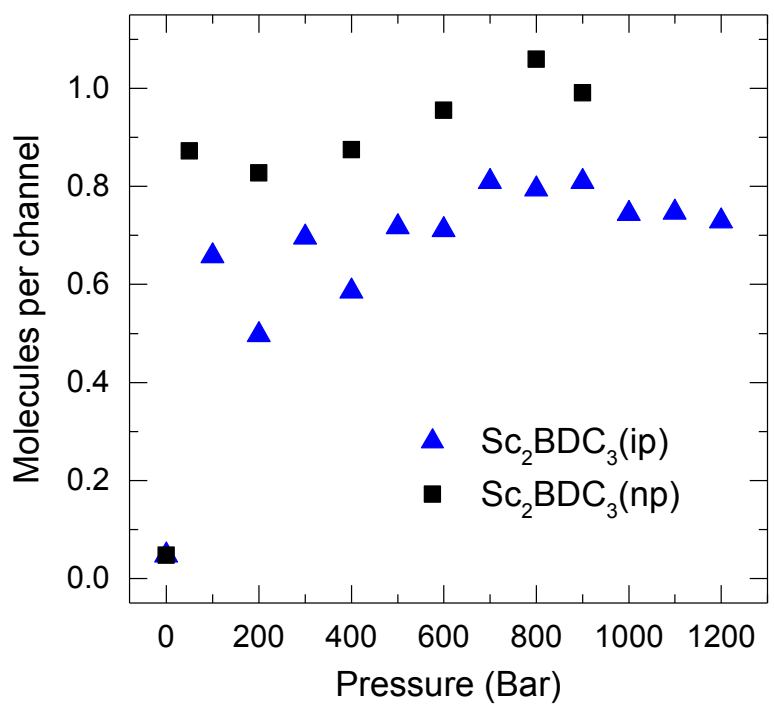

Figure 4.7 - Number of molecules channel for both $\mathrm{Sc}_{2} \mathrm{BDC}_{3}(\mathrm{ip})$, blue triangles, and $\mathrm{Sc}_{2} \mathrm{BDC}_{3}(\mathrm{np})$, black squares, with increasing pressure.

increasing pressure occurred before a pressure of 4000 bar was reached, as shown in the previous study. The maximum uptake of 0.8 molecules per channel calculated here, also matches very well with the previous high-pressure DAC experiments where the uptake of isopentane at 4 kbar equated to 0.87 molecules per channel, Table 4.1. ${ }^{3}$

\subsubsection{The effect on $\mathrm{Sc}_{2} \mathrm{BDC}_{3}$ on uptake of $n$-pentane}

The same approach for monitoring structural changes was used for $\mathrm{Sc}_{2} \mathrm{BDC}_{3}(\mathrm{np})$ however the effect of uptake of $n$-pentane were much larger than that seen in $\mathrm{Sc}_{2} \mathrm{BDC}_{3}(\mathrm{ip})$. Again, the motion of the phenyl ring of the Group-2 $\mathrm{BDC}$ linker was monitored through the principal axes of the ADPs for the carbon atom C7, Figure 4.8 , and the uptake of $n$-pentane monitored using the SQUEEZE algorithm, Figure 4.7.

On increasing the pressure from ambient to 200 bar there was an immediate large uptake of $n$-pentane into the channels, increasing to 0.83 molecules per channel at 200 bar, Figure 4.7. On increasing pressure further, to $400 \mathrm{bar}$, the principal axis of the ADP for C7 measured $0.29 \AA^{2}$. This degree of distortion of the ADP's on the Group-2 BDC ligands was not observed in $\mathrm{Sc}_{2} \mathrm{BDC}_{3}(\mathrm{ip})$ until 1200 bar. The $\mathrm{BDC}$ ligand in $\mathrm{Sc}_{2} \mathrm{BDC}_{3}(\mathrm{np})$ therefore had to be modelled in two positions (50:50 
split) immediately on applying pressure to 400 bar. On increasing the pressure further, the angle of rotation quickly increased from $13.4^{\circ}$ at 400 bar to $28.7^{\circ}$ at 800 bar. On increasing pressure to $900 \mathrm{bar}$, the angle of rotation for the 50:50 split model exceeded $30^{\circ}$. Atoms in both disordered phenyl groups became very prolate. As a consequence, at 900 bar the C7-phenyl atom was split to give a 25:25:25:25 four component disorder to better represent the motion of the phenyl ring.

The angle of rotation of the phenyl ring at 900 bar was calculated to be $49.3^{\circ}$. This was much larger than the rotation seen at 2 kbar $\left(20.6^{\circ}\right)$, during previous highpressure DAC experiments and was more comparable to that observed at $5 \mathrm{kbar}$ $\left(43.7^{\circ}\right)$.

From the SQUEEZE output, as seen in $\mathrm{Sc}_{2} \mathrm{BDC}_{3}(\mathrm{np})$, on increasing pressure to 200 bar, a sudden increase in electron density in the pores was observed, Figure 4.7. On increasing pressure further to 900 bar, the electron density continued to increase (with some fluctuation) equating to a maximum of 1.1 molecules per channel. The SQUEEZE result, together with the rotation of the Group-2 phenyl group observed on increasing pressure, again confirmed that in $\mathrm{Sc}_{2} \mathrm{BDC}_{3}$ (ip) uptake of isopentane occurred before 4000 bar, as observed in the previous DAC study.

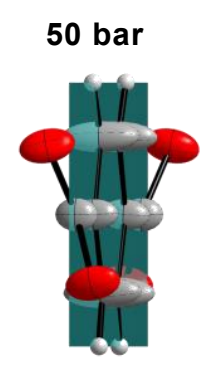

-

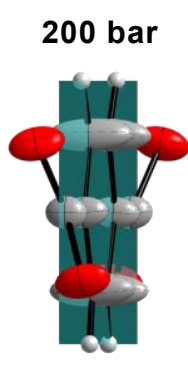

$-$

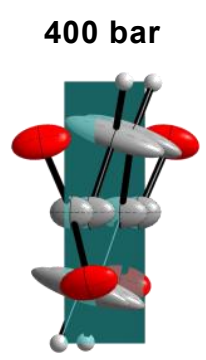

$13.4^{\circ}$

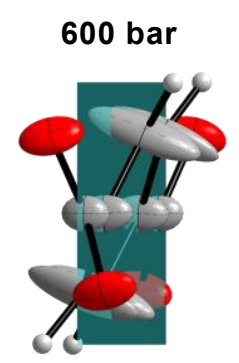

$21.8^{\circ}$

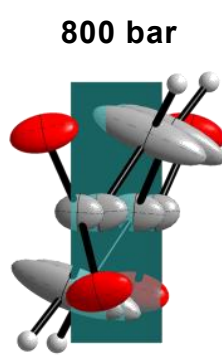

$28.7^{\circ}$

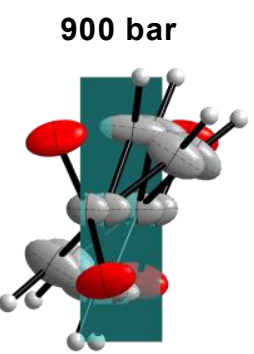

$49.3^{\circ}$

Figure 4.8 - The effect of uptake of n-pentane with increasing pressure upon the phenyl group in the Group-2 BDC linker. Only half of the disordered phenyl rings are shown in each for clarity. Non-hydrogen atoms are modelled with $50 \%$ probability ellipsoids and the (001) plane shown in green. 


\subsection{Conclusions}

We have, for the first time, used a sapphire capillary pressure cell to analyse and follow the structural behaviour of a small pore $\mathrm{MOF}\left(\mathrm{Sc}_{2} \mathrm{BDC}_{3}\right)$ below 1500 bar when exposed to $n$-pentane and isopentane. The only difference between these two hydrocarbons is the shape and kinetic diameter of the molecules, Table 4.1.

On increasing pressure surrounding the crystal with $n$-pentane, one of the BDC phenyl groups' twisted, caused by the uptake of $n$-pentane molecules into the pores. This was also observed in isopentane, along with uptake of isopentane molecules into the pores. The longer chain and increased flexibility of $n$-pentane, in comparison to isopentane, allowed for a greater uptake of the solvent into the channels. As the pressure was increased, the $n$-pentane superfilled the channels resulting in swelling of the structure, a feature not clearly observed in previous high-pressure DAC experiments. Swelling is a known important stabilisation phenomenon found in MOFs at pressure, and confirmed the high-pressure stability for $\mathrm{Sc}_{2} \mathrm{BDC}_{3}(\mathrm{np})$ at elevated pressures. ${ }^{8-11}$

Isopentane on the other hand, has a larger kinetic diameter than $n$-pentane, and lowers the uptake of this solvent into channels. Without the stabilising effect of the initial swelling and subsequent superfilling of the channels $\mathrm{Sc}_{2} \mathrm{BDC}_{3}(\mathrm{ip})$ is much more compressible. This in turn greatly reduced the high-pressure stability.

The uptake of isopentane below 1200 bar, observed here for the first time, also resulted in the rotation of the phenyl ring of the Group-2 BDC linker, however to a much lesser extent than seen with $n$-pentane $\left(14.9^{\circ}\right.$ at $\left.1200 \mathrm{bar}\right)$.

Previous experiments on $\mathrm{Sc}_{2} \mathrm{BDC}_{3}$ have shown that the MOF shows promise as a stationary phase for gas chromatography (GC). ${ }^{12}$ In this previous study, a packed column of $\mathrm{Sc}_{2} \mathrm{BDC}_{3}$ could separate hydrocarbons with similar boiling points (Figure 4.9). The retention time for methylcyclohexane, for example, was found to be $70 \%$ longer than that for $n$-heptane. The boiling points for these two compounds differ by only $3{ }^{\circ} \mathrm{C}$.

The high-pressure work presented here and the initial work on GC suggest the possible use of $\mathrm{Sc}_{2} \mathrm{BDC}_{3}$ as the adsorbent in ultra-high-pressure liquid 
chromatography (UHPLC) systems. UHPLC can be run at pressure up to 1400 bar. ${ }^{13}$ As shown here, these pressures could easily be high enough to cause the uptake of oversized guest into $\mathrm{Sc}_{2} \mathrm{BDC}_{3}$ and this pressure dependant response could have a beneficial effect on the resolving power when separating mixtures.

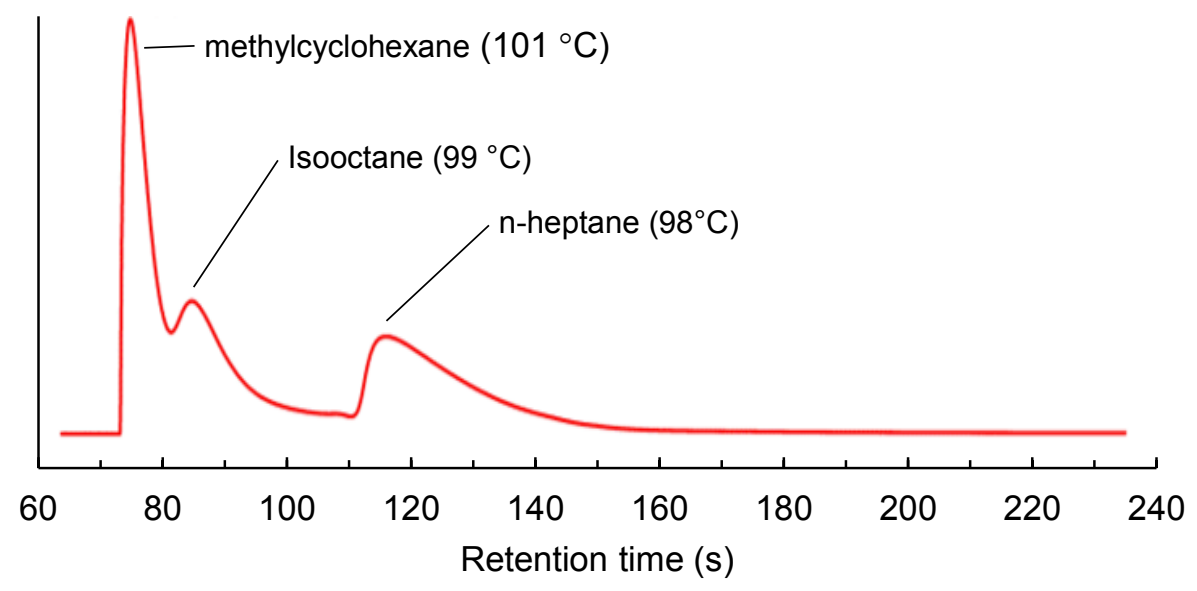

Figure 4.9 - GC traces for an alkane mixture of similar boiling points but differing size.

Figure adapted from Mowat, 2012. 


\subsection{References}

1 Miller, S. R., Wright, P. A., Serre, C., Loiseau, T., Marrot, J. \& Ferey, G. A microporous scandium terephthalate, $\mathrm{Sc}_{2}\left(\mathrm{O}_{2} \mathrm{CC}_{6} \mathrm{H}_{4} \mathrm{CO}_{2}\right)_{3}$, with high thermal stability. Chemical Communications, 3850-3852, doi:10.1039/B506677H (2005).

2 Miller, S. R., Wright, P. A., Devic, T., Serre, C., Férey, G., Llewellyn, P. L., Denoyel, R., Gaberova, L. \& Filinchuk, Y. Single Crystal X-ray Diffraction Studies of Carbon Dioxide and Fuel-Related Gases Adsorbed on the Small Pore Scandium Terephthalate Metal Organic Framework, $\mathrm{Sc}_{2}\left(\mathrm{O}_{2} \mathrm{CC}_{6} \mathrm{H}_{4} \mathrm{CO}_{2}\right)_{3}$. Langmuir 25, 3618-3626, doi:10.1021/la803788u (2009).

3 McKellar, S. C., Sotelo, J., Greenaway, A., Mowat, J. P. S., Kvam, O., Morrison, C. A., Wright, P. A. \& Moggach, S. A. Pore Shape Modification of a Microporous Metal-Organic Framework Using High Pressure: Accessing a New Phase with Oversized Guest Molecules. Chemistry of Materials 28, 466-473, doi:10.1021/acs.chemmater.5b02891 (2016).

4 Nowell, H., Barnett, S. A., Christensen, K. E., Teat, S. J. \& Allan, D. R. I19, the small-molecule single-crystal diffraction beamline at Diamond Light Source. Journal of Synchrotron Radiation 19, 435-441, doi:10.1107/s0909049512008801 (2012).

5 CrysAlis PRO (Agilent, Agilent Technologies Ltd, Yarnton, Oxfordshire, England., 2014).

6 Betteridge, P. W., Carruthers, J. R., Cooper, R. I., Prout, K. \& Watkin, D. J. CRYSTALS version 12: software for guided crystal structure analysis. Journal of Applied Crystallography 36, 1487-1487, doi:10.1107/s0021889803021800 (2003).

7 Spek, A. L. PLATON SQUEEZE: a tool for the calculation of the disordered solvent contribution to the calculated structure factors. Acta Crystallographica Section C 71, 9-18, doi:10.1107/s2053229614024929 (2015).

8 Graham, A. J., Banu, A.-M., Dueren, T., Greenaway, A., McKellar, S. C., Mowat, J. P. S., Ward, K., Wright, P. A. \& Moggach, S. A. Stabilization of 
Scandium Terephthalate MOFs against Reversible Amorphization and Structural Phase Transition by Guest Uptake at Extreme Pressure. Journal of the American Chemical Society 136, 8606-8613, doi:10.1021/ja411934f (2014).

9 Graham, A. J., Allan, D. R., Muszkiewicz, A., Morrison, C. A. \& Moggach, S. A. The Effect of High Pressure on MOF-5: Guest-Induced Modification of Pore Size and Content at High Pressure. Angewandte Chemie International Edition 50, 11138-11141, doi:10.1002/anie.201104285 (2011).

10 Graham, A. J., Tan, J.-C., Allan, D. R. \& Moggach, S. A. The effect of pressure on $\mathrm{Cu}$-btc: framework compression vs. guest inclusion. Chemical Communications 48, 1535-1537, doi:10.1039/C1CC16045A (2012).

11 Moggach, S. A., Bennett, T. D. \& Cheetham, A. K. The Effect of Pressure on ZIF-8: Increasing Pore Size with Pressure and the Formation of a HighPressure Phase at $1.47 \mathrm{GPa}$. Angewandte Chemie International Edition 48, 7087-7089, doi:10.1002/anie.200902643 (2009).

12 Mowat, J. P. S. Synthesis, characterisation and adsorption properties of metal-organic frameworks and the structural response to functionalisation and temperature Doctor of Philosophy thesis, University of St Andrews, (2012).

13 Dong, M. W. \& Zhang, K. Ultra-high-pressure liquid chromatography (UHPLC) in method development. TRAC Trends in Analytical Chemistry 63, 21-30, doi:https://doi.org/10.1016/j.trac.2014.06.019 (2014). 


\section{- Chapter 5 - \\ The Effect of High Pressure on a \\ Cu-Based Framework}




\subsection{Introduction}

High-pressure crystallographic experiments over the last 20 years or so have proved to be a unique tool in probing the mechanical properties of the organic solid state, ${ }^{1}$ metal-complexes, and 2D/3D coordination compounds. ${ }^{2}$ In particular, high-pressure techniques have been used to study a vast array of mechanical and chemical properties, such as monitoring changes in electrical conductivity, ${ }^{3}$ amorphisation, ${ }^{4}$ post-synthetic modification ${ }^{5}$ and chemical reactions such as polymerisation $^{6}$ and cycloaddition chemistry, ${ }^{7}$ where reaction rates can be increased substantially. Previous high-pressure experiments on porous metalorganic framework (MOF) materials have shown that on loading a diamond anvil cell (DAC) with a single-crystal or polycrystalline powder, the hydrostatic medium which surrounds the sample (to ensure hydrostatic conditions) can be forced inside the pores on increasing pressure, causing the pore and sample volume to increase on increasing pressure. ${ }^{8}$ More recently, this technique has also been used to determine the position of $\mathrm{CH}_{4}$ and $\mathrm{CO}_{2}$ molecules inside the pores of a small pore Sc-based MOF at room temperature using a laboratory X-ray diffractometer, and has proved useful in experimentally determining the maximum size of guest molecule which can penetrate into a pore. ${ }^{9}$ On direct compression of more dense frameworks, negative linear compressibility (NLC) has also been observed, which results in an expansion of one or more of the unit cell dimensions, but an overall contraction in volume. Such changes in the compressibility behaviour of metalcontaining framework materials is usually as a result of common structural motifs which rotate or bend in such a way as to increase the length of certain crystallographic axes. ${ }^{10}$ Changes in coordination environment can also be induced at pressure, as metal-ligand bonds are more susceptible to compression than covalent bonds. ${ }^{2}$ In previous high pressure studies on metal complexes or coordination compounds in which the metal ion has an asymmetric octahedral environment caused by Jahn-Teller (JT) distortions for example (such as those observed in $\mathrm{Cu}^{2+}$ and $\mathrm{Mn}^{3+}$ complexes), the application of pressure can result in compression of the JT axis, and can even be switched to lie along another bonding direction within the octahedron. ${ }^{11,12}$ 
Here we present a high-pressure crystallographic study on a previously unreported $\mathrm{Cu}$-framework bis[1-(4-pyridyl)butane-1,3-dione]copper(II) (hereafter referred to as CuPyr-I). On application of pressure, CuPyr-I demonstrates several of these phenomena simultaneously within the same framework, including a single-crystal to single-crystal phase transition, a switching of the JT axis which is dependent on the hydrostatic media, piezochromism and NLC behaviour. 


\subsection{Experimental}

Note: Synthesis of Bis[1-(4-pyridyl)butane-1,3-dione]copper(II), CuPyr-I was carried out by Dr Priyanka Comar and Dr Sergio Sanz from the University of Edinburgh.

\subsubsection{Synthesis}

Bis[1-(4-pyridyl)butane-1,3-dione]copper(II) $\left[\mathrm{Cu}\left(\mathrm{C}_{9} \mathrm{H}_{8} \mathrm{NO}_{2}\right)_{2}\right]$ (hereafter referred to as CuPyr-I), was synthesised from $\mathrm{CuSO}_{4}(0.2 \mathrm{mmol})$ dissolved in DCM (10 mL) and added to a solution of $\mathrm{GdL}_{3}(0.2 \mathrm{mmol}$, where $\mathrm{HL}=1$-(4-pyridyl) butane-1,3dione) in $\mathrm{MeOH}(10 \mathrm{~mL})$. The reaction mixture was stirred for $1 \mathrm{hr}$ before being filtered and diffused from diethyl ether. Green hexagonal prism crystals formed after 4 days suitable for single crystal X-ray diffraction.

\subsubsection{Single-Crystal X-ray Diffraction Experiments}

Ambient and variable temperature single-crystal data were collected on a threecircle Bruker SMART APEXII diffractometer with graphite monochromated $\mathrm{MoK}_{\alpha}$ radiation $(\lambda=0.71073 \AA$ ) . Short data collections were performed in order to index the crystal on cooling from $300 \mathrm{~K}$ to $150 \mathrm{~K}$, at $50 \mathrm{~K}$ steps, and at room temperature for face indexing. Full data collections were made at room temperature and $120 \mathrm{~K}$. Unit cell indexing, face indexing and data processing were carried out using the Bruker APEX II software. Integration was performed using the program SAINT while the absorption corrections were carried out using the program SADABS. ${ }^{13,14}$

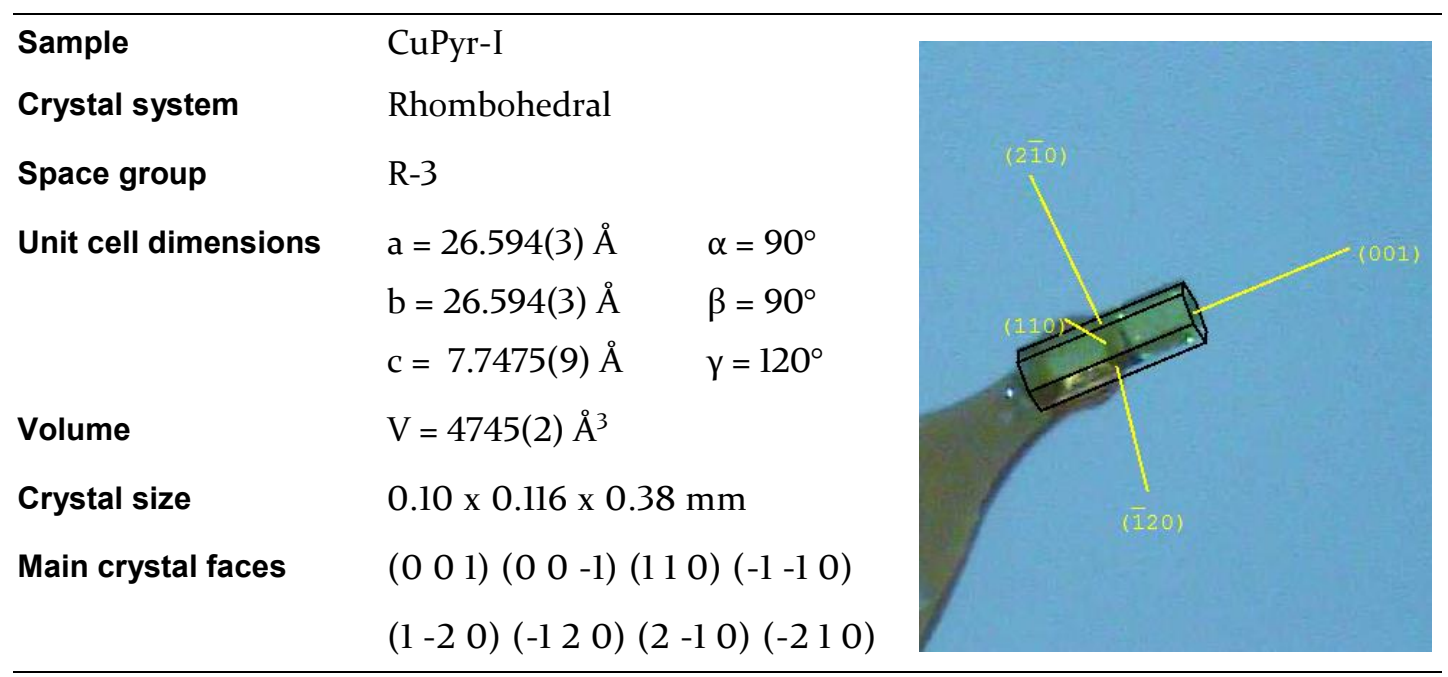

Figure 5.1 - Summary of CuPyr-I crystallographic and face indexing information at room temperature. 
High-pressure single-crystal diffraction data were obtained using the set-up described in Chapter 2. High pressure experiments were performed using a Merrill-Bassett diamond anvil cell (DAC) with a half opening angle of $40^{\circ}$ equipped with $600 \mu \mathrm{m}$ culet Boehler-Almax cut diamonds, tungsten-carbide backing discs and a tungsten gasket. ${ }^{15,16}$ A single crystal of the sample with a small chip of ruby (as the pressure calibrate) were loaded into the DAC using either methanol $(\mathrm{MeOH})$, Fluorinert (FC-70) or $n$-pentane as a hydrostatic media. The ruby fluorescence method was used to measure the pressure throughout the experiment. $^{17}$

High pressure measurements using FC-70 as a hydrostatic medium were collected on a Bruker SMART APEXII diffractometer with graphite monochromated $\mathrm{MoK}_{\alpha}$ radiation $(\lambda=0.71073 \AA$ ). Data collection strategies were as described by Dawson et al., with a step size of $0.5^{\circ}$ and exposure time of $40 \mathrm{~s}$ (60s for the last pressure point). ${ }^{18}$ The data were collected from 0.07 to $1.56 \mathrm{GPa}$. Unit cell indexing and data processing were carried out using the Bruker APEX II software. Integration was performed using SAINT using dynamic masks created to remove regions of the detector which were shaded by the pressure cell. The absorption corrections were carried out using SADABS. ${ }^{13,14,19}$ The same procedure was used to collect high-pressure data using $\mathrm{MeOH}$ as a hydrostatic media from 0.34 to $2.70 \mathrm{GPa}$, and $n$-pentane from 0.29 to $1.61 \mathrm{GPa}$.

High-pressure measurements using $\mathrm{MeOH}$ as a hydrostatic medium were also collected at the Diamond Light Source on beamline I19-EHl using synchrotron radiation $(\lambda=0.48590 \AA$ ) on a four-circle Crystal Logic diffractometer equipped with a Rigaku Saturn 724+CCD detector. Data collection strategies were based on those described by Dawson et al., with a step size of $0.5^{\circ}$ and exposure time of 1 s. ${ }^{18}$ Unit cell indexing and data processing were carried out using the Bruker APEX II software. ${ }^{19}$ Integrations were performed using SAINT and absorption corrections were carried out using SADABS. ${ }^{13,14}$ The data were integrated using dynamic masks to not only remove regions of the detector which were shaded by the pressure cell, but also to mask intense powder rings from the tungsten gasket. Data were collected in $\mathrm{MeOH}$ from 0.52 to $5.40 \mathrm{GPa}$. 
An initial structure was determined by direct methods using ShelXT. ${ }^{20}$ This same model was then used to refine against the high pressure data. Merging and refinement of the data were performed using CRYSTALS. ${ }^{21}$ All 1,2 and 1,3 distances for the ligand were restrained to the values obtained from the ambient-pressure structure determination. All metal-ligand distances and angles were allowed to refine freely, as were all torsion angles. Residual electron density in the pores was modelled using the SQUEEZE algorithm in PLATON.

\subsubsection{Powder Diffraction Measurements}

Variable temperature X-ray powder diffraction data were collected on CuPry-I from $300 \mathrm{~K}$ to $180 \mathrm{~K}$ in $10 \mathrm{~K}$ steps, then from $180 \mathrm{~K}$ to $130 \mathrm{~K}$ in $5 \mathrm{~K}$ steps. The sample was loaded into a $0.7 \mathrm{~mm}$ glass capillary, and mounted on a Bruker D8 diffractometer using $\mathrm{Cu} \mathrm{K} \alpha_{1}$ radiation $(\lambda=1.54056 \AA$ ). Data were collected from $5-50^{\circ}$ in $2 \theta$ in $0.02^{\circ}$ steps.

\subsubsection{Thermogravimetric analysis (TGA)}

TGA was carried out on CuPyr-I to assess its thermal stability. A heating rate of $10^{\circ} \mathrm{C} / \mathrm{min}$ was applied up to a final temperature of $550{ }^{\circ} \mathrm{C}$ in an inert atmosphere of nitrogen at a flow rate of $50 \mathrm{ml} / \mathrm{min}$ on a Netzsch STA $449 \mathrm{Fl}$ fitted with a platinum furnace. 


\subsection{Results and Discussion}

Under ambient temperature and pressure CuPyr-I crystallises in the rhombohedral space group $R-3(a, b=26.594(3) \AA, c=7.7475(9) \AA)$, Figure 5.1. Each $\mathrm{Cu}$-centre is coordinated to four 1-(4-pyridyl)butane-1,3-dione linkers, two of these ligands are bound through the dione $\mathrm{O}$-atoms, with the remaining two binding through the $\mathrm{N}$-atom of the pyridine ring to form a 3D polymer (Figure 5.2). The crystal structure of CuPyr-I is composed of an interpenetration of these $3 \mathrm{D}$ polymers to form one-dimensional porous channels $(\approx 2 \AA$ in diameter) that run along the c-axis direction (Figure 5.2).

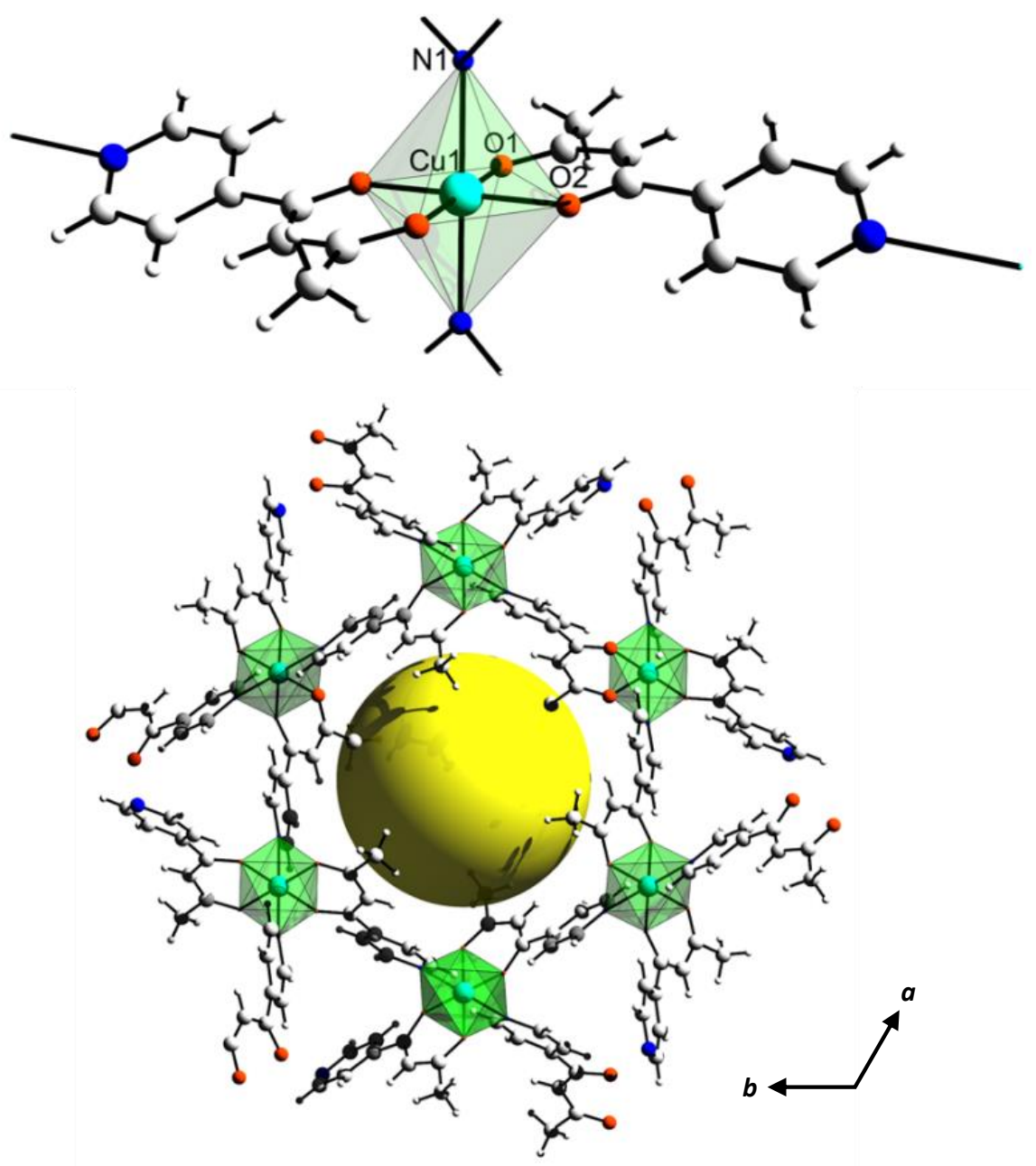

Figure 5.2 - Ball and stick model showing the coordination environment around the $\mathrm{Cu}^{2+}$ ion in $\mathrm{CuPyr}-\mathrm{I}$, and 3D-pore structure as viewed along the c-axis direction. The yellow sphere represents the available pore-space. Colour scheme is red: Oxygen, blue: Nitrogen, black: Carbon, white: Hydrogen and cyan: Copper. The $\mathrm{Cu}^{2+}$ octahedron is drawn in green. 


\subsubsection{High Pressure Experiments}

To directly compress CuPyr-I Fluorinert FC-70, a mixture of large perfluorinated hydrocarbons, was selected as a hydrostatic medium. On increasing pressure from 0.07 to $1.56 \mathrm{GPa}$ compression of the framework occurs, resulting in a $9.89 \%$ reduction in volume, while the $a / b$-axes and $c$-axis reduced by $4.46 \%$ and $1.25 \%$ respectively (Figure 5.3 (blue triangles), Table 5.1).

On increasing pressure to $1.84 \mathrm{GPa}$, the framework became amorphous, though this is unsurprising as the hydrostatic limit for FC-70 is $\approx 2 \mathrm{GPa}$, and compression of frameworks in non-hydrostatic conditions usually results in amorphisation. ${ }^{22,23}$
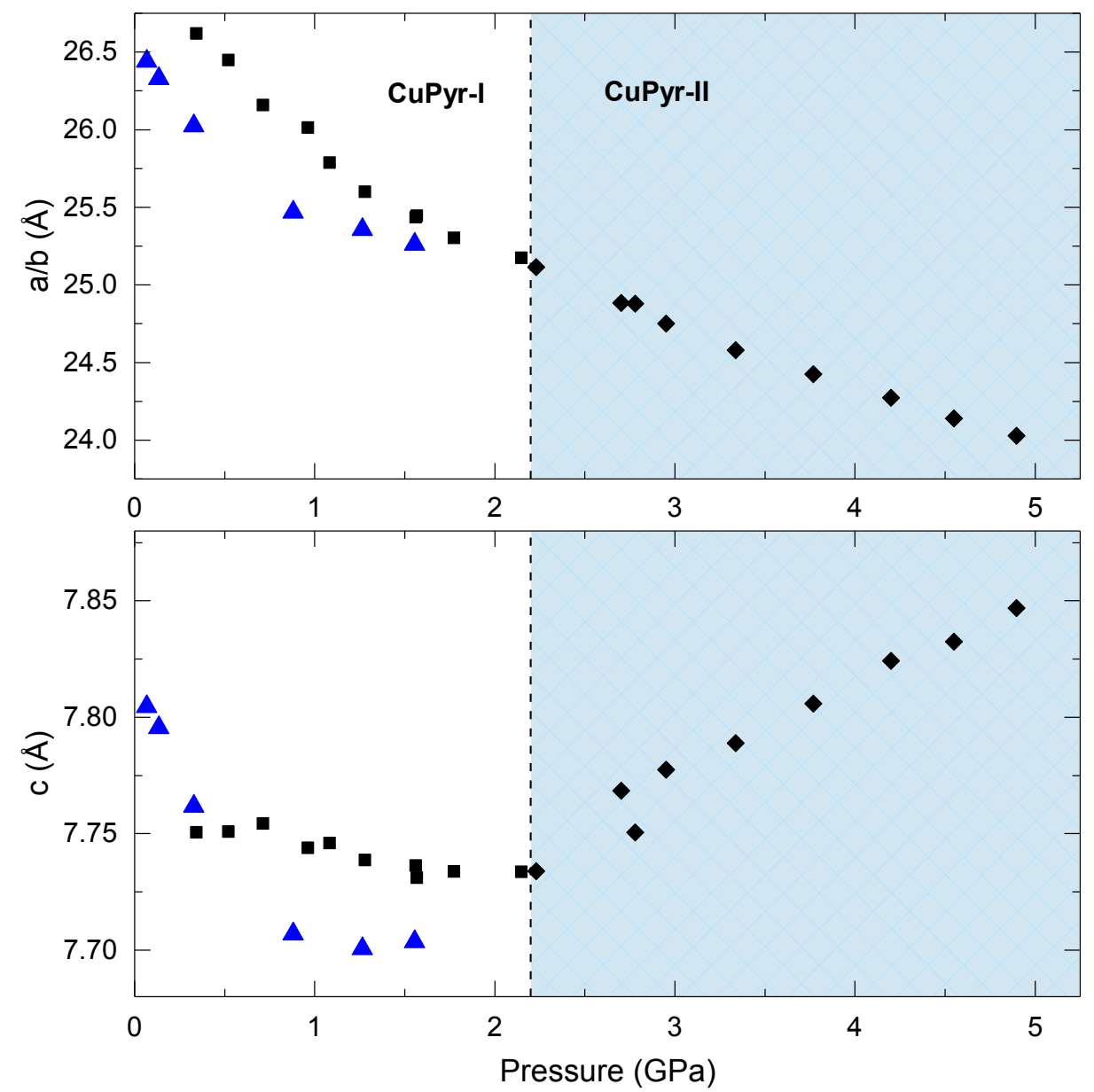

Figure $5.3-a / b$ and $c$-axes as a function of pressure. Compression using FC-70 is shown in triangles and $\mathrm{MeOH}$ in squares and diamonds for CuPyr-I to CuPyr-II respectively. The values for the $a / b$ axes in CuPyr-II have been halved to allow the direct comparison to the compression seen in CuPyr-I. The vertical dashed line indicates the phase transition above $2.15 \mathrm{GPa}$. 
On increasing pressure to $1.56 \mathrm{GPa}$, the three-symmetry independent $\mathrm{Cu}-\mathrm{O} / \mathrm{N}$ bond lengths to the ligand were monitored (Figure 5.4, Table 5.5). Under ambient pressure conditions, the two $\mathrm{Cu}-\mathrm{Nl}$ pyridine bonds are longer than the four $\mathrm{Cu}$ Ol/O2 dione bonds, typical for a JT distorted $\mathrm{Cu}^{2+}$ complex. However, on increasing pressure the direction of the JT axis gradually changed from $\mathrm{Cu}-\mathrm{N} 1$ to the $\mathrm{Cu}$-Ol bond (the dione oxygen in the 3-position), becoming equidistant at $\approx 0.57 \mathrm{GPa}$. By $1.56 \mathrm{GPa}$ the lengths of the $\mathrm{Cu}-\mathrm{Nl}$ and $\mathrm{Cu}-\mathrm{Ol}$ bonds had steadily reduced and increased by $12.3 \%$ and $8.9 \%$, respectively. Throughout this the $\mathrm{Cu}$ $\mathrm{O} 2$ bond remained essentially unchanged.

Pressure induced JT switching has been observed in other systems, including a $\mathrm{Mn}_{12}$ single-molecule magnet cluster that re-orientates the JT axis on one of the Mn centres at 2.5 GPa. $^{11}$ A similar transition was also observed in $\left[\mathrm{CuF}_{2}\left(\mathrm{H}_{2} \mathrm{O}\right)_{2}(\mathrm{pyz})\right]$ (pyz=pyrazine), where the JT axis was reoriented from the $\mathrm{Cu}$ $\mathrm{N}$ bond to the perpendicular $\mathrm{Cu}-\mathrm{O}$ bond, though this occurs during a phase transition at $1.1 \mathrm{GPa} .{ }^{24}$ Here, in CuPyr-I, no phase transition takes place, and unusually the JT switching appears to occur progressively on increasing pressure. ${ }^{12}$

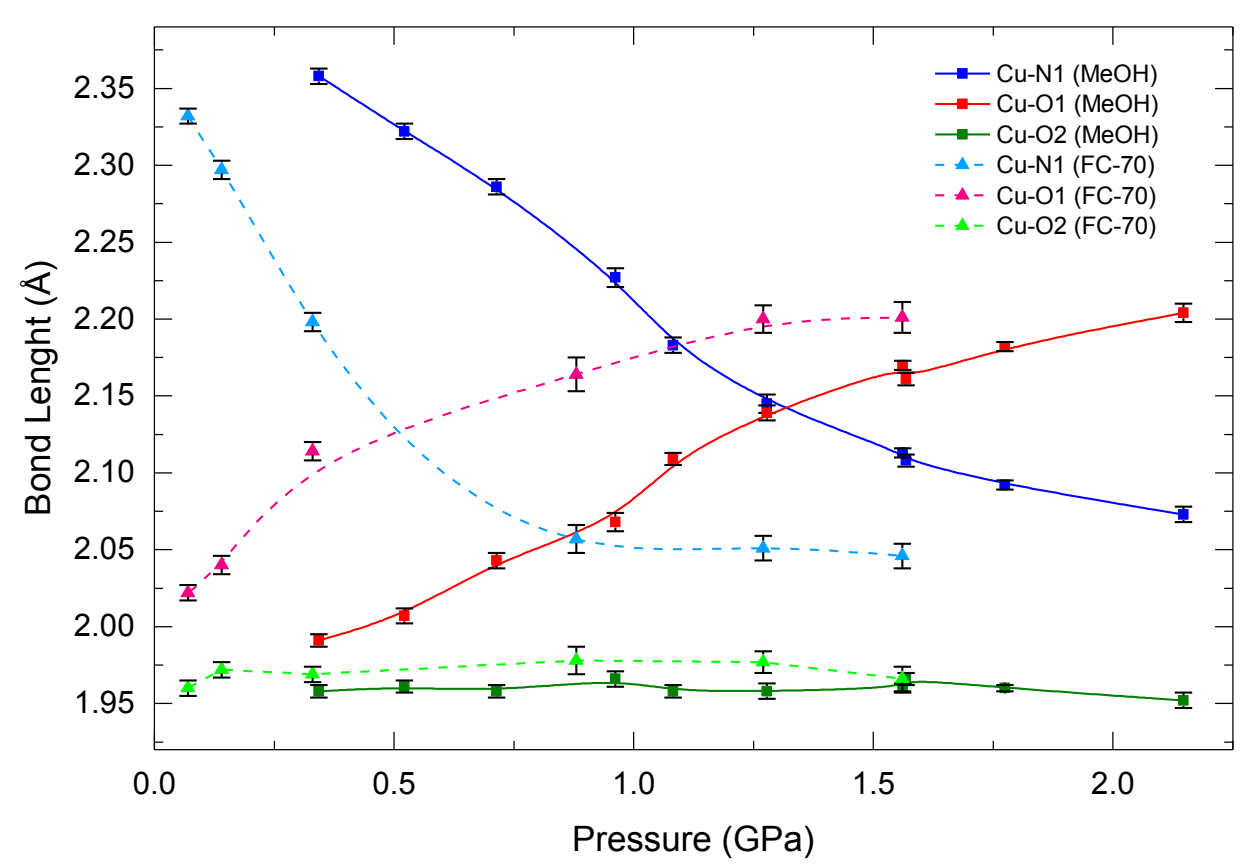

Figure $5.4-\mathrm{Cu}-\mathrm{Ol}, \mathrm{Cu}-\mathrm{Nl}$ and $\mathrm{Cu}-\mathrm{O} 2$ bond lengths on increasing pressure in both FC70 (triangles, dashed lines) and $\mathrm{MeOH}$ (squares, solid lines). 
Using methanol (MeOH) as a hydrostatic medium, CuPyr-I was compressed in two separate experiments. The first, from 0.52 to $5.28 \mathrm{GPa}$, was collected using synchrotron radiation, and the second, from 0.34 to $2.95 \mathrm{GPa}$, was collected using a laboratory X-ray source. On increasing pressure to $2.15 \mathrm{GPa}$, the $a / b$ and $c$-axes compressed by $6.22 \%$ and $0.39 \%$, respectively (Figure 5.3, Table 5.2 and Table 5.3). On increasing pressure from 2.15 to $2.78 \mathrm{GPa}$, CuPyr-I underwent a singlecrystal to single-crystal isosymmetric phase transition to a previously unobserved phase (hereafter referred to as CuPyr-II). The transition to CuPyr-II resulted in a doubling of the $a / b$-axes, whilst the $c$-axis remained essentially unchanged. This could be easily observed from calculated precession images, as extra reflections could be observed along the [100] and [010] directions as a result of the doubling of the $a / b$-axes (Figure 5.5). On increasing the pressure further, the $a / b$-axes continued to be compressed, however the c-axis increased in length, exhibiting negative linear compressibility (NLC) until the sample became amorphous at 5.28 GPa. The data were of poor quality after the phase transition, and only the connectivity of the CuPyr-II phase could be determined at 3.34 GPa. Above 3.34 GPa, only unit cell dimensions could be extracted.

The occurrence of positive linear compressibility (PLC) followed by NLC is unusual in a framework material, and we could find only a few examples in the literature where this occurs. ${ }^{25,26}$ During the NLC, the $c$-axis expanded by $1.46 \%$, to give a compressibility of $K_{\mathrm{NLC}}=-5.3(0.8) \mathrm{TPa}^{-1}(\Delta p=2.23-4.90 \mathrm{GPa}) . K_{\mathrm{NLC}}$ is calculated using the relationship $K=-1 / l(\partial l / \partial p)_{T}$, where $l$ is the length of the

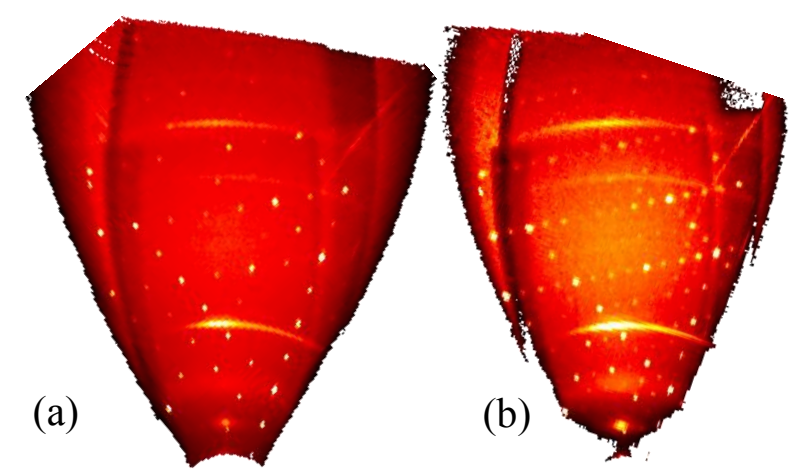

Figure 5.5 - Precession image of the $h k 0$ plane at (a) $1.77 \mathrm{GPa}$ and (b) $2.23 \mathrm{GPa}$ using $\mathrm{MeOH}$ as a hydrostatic media. 
axis and $\partial p$ is the change in pressure at constant temperature. ${ }^{27}$ The value of $K_{\mathrm{NLC}}$ here is rather small compared to the massive NLC behaviour observed in the low pressure phase of $\mathrm{Ag}_{3}\left[\mathrm{Co}(\mathrm{CN})_{6}\right]^{7}\left(K_{\mathrm{NLC}}=-76(9) \mathrm{TPa}^{-1}, \Delta p=0-0.19 \mathrm{GPa}\right)$ or the flexible MOF MIL-53(Al) $\left(K_{\mathrm{NLC}}=-28 \mathrm{TPa}^{-1}, \Delta p=0-3 \mathrm{GPa}\right)$ for example, ${ }^{23}$ and is much more comparable to the dense $\mathrm{Zn}$ formate MOF $\left[\mathrm{NH}_{4}\right]\left[\mathrm{Zn}(\mathrm{HCOO})_{3}\right]$, by Cheetham et al., (-1.8(8) $\left.\mathrm{TPa}^{-1}(\Delta p=0-0.94 \mathrm{GPa})\right) .{ }^{28}$

On increasing pressure using $\mathrm{MeOH}$, the JT axis was again supressed on compression, with the $\mathrm{Cu}-\mathrm{N} 1$ bond reducing in length by $0.288 \AA$ (12\%) between 0.34 and $2.15 \mathrm{GPa}$, while the $\mathrm{Cu}-\mathrm{O} 1$ bond length increased by $0.216 \AA$ (11\%). The pressure at which $\mathrm{Cu}-\mathrm{Nl}$ and $\mathrm{Cu}-\mathrm{Ol}$ became equidistant was $1.28 \mathrm{GPa}$, measuring 2.140(5) $\AA$ and 2.131(6) $\AA$ respectively (Figure 5.4, Table 5.6). Across the entire pressure range, little to no compression or expansion was observed in the $\mathrm{Cu}-\mathrm{O} 2$ bond in the 1-positon of the dione in CuPyr-I, the same trend observed when compressed in FC-70. The JT switching pressure in $\mathrm{MeOH}$ however was $0.71 \mathrm{GPa}$ higher than observed by direct compression in FC-70 (0.57 GPa) with the JT switching maintained in CuPyr-II at 3.34 GPa (Table 5.6). This, to our knowledge, is the first time that pressure induced JT switching has been observed to be dependent on the hydrostatic medium.

Table 5.1 - Unit cell dimensions, volume, SQUEEZE output, including the average void volume and number of electrons per pore as a function of pressure using FC-70 as a hydrostatic liquid. The ambient data set is given for comparison.

\begin{tabular}{ccccccc}
\hline Phase & $\begin{array}{c}\text { Pressure } \\
(\mathbf{G P a})\end{array}$ & $\mathbf{a} / \mathbf{b}(\boldsymbol{A})$ & $\boldsymbol{c}(\boldsymbol{A})$ & $\begin{array}{c}\text { Volume } \\
\left(\boldsymbol{A}^{3}\right)\end{array}$ & $\begin{array}{c}\text { Average void } \\
\text { volume }\left(\boldsymbol{A}^{3}\right)\end{array}$ & $\begin{array}{c}\text { electrons/ } \\
\text { void }\end{array}$ \\
\hline CuPyr-I & ambient & $26.594(3)$ & $7.7475(9)$ & $4745.1(12)$ & 384 & 112 \\
CuPyr-I & 0.07 & $26.4400(6)$ & $7.8045(2)$ & $4725.0(2)$ & 388 & 112 \\
CuPyr-I & 0.14 & $26.3304(7)$ & $7.7955(2)$ & $4680.5(3)$ & 380 & 112 \\
CuPyr-I & 0.33 & $26.0243(5)$ & $7.7622(2)$ & $4552.7(2)$ & 353 & 119 \\
CuPyr-I & 0.88 & $25.469(2)$ & $7.7069(6)$ & $4329.4(6)$ & 319 & 110 \\
CuPyr-I & 1.27 & $25.342(2)$ & $7.7011(5)$ & $4283.1(8)$ & 312 & 138 \\
CuPyr-I & 1.56 & $25.258(1)$ & $7.7047(4)$ & $4256.4(4)$ & 313 & 126
\end{tabular}


Table 5.2 - Unit cell dimensions, volume, SQUEEZE output, including the average void volume and number of electrons per pore as a function of pressure using $\mathrm{MeOH}$ as a hydrostatic liquid, collected using synchrotron radiation.

\begin{tabular}{ccccccc}
\hline Phase & $\begin{array}{c}\text { Pressure } \\
(\mathbf{G P a})\end{array}$ & $\mathbf{a} / \mathbf{b}(\mathbf{A})$ & $\boldsymbol{c}(\mathbf{A})$ & $\begin{array}{c}\text { Volume } \\
\left(\AA^{3}\right)\end{array}$ & $\begin{array}{c}\text { Average void } \\
\text { volume }\left(\AA^{3}\right)\end{array}$ & $\begin{array}{c}\text { electrons/ } \\
\text { void }\end{array}$ \\
\hline CuPyr-I & 0.52 & $26.453(2)$ & $7.7499(6)$ & $4696.5(8)$ & 397 & 173 \\
CuPyr-I & 0.96 & $26.025(2)$ & $7.7498(7)$ & $4545.7(8)$ & 371 & 174 \\
CuPyr-I & 1.57 & $25.4451(17)$ & $7.7312(5)$ & $4335.0(6)$ & 336 & 141 \\
CuPyr-I & 2.15 & $25.1855(18)$ & $7.7300(6)$ & $4246.3(6)$ & 341 & 129 \\
CuPyr-II & 2.78 & $49.757(3)$ & $7.7506(5)$ & $16618(2)$ & N/A & N/A \\
CuPyr-II & 3.34 & $49.109(3)$ & $7.7842(5)$ & $16258(2)$ & N/A & N/A \\
CuPyr-II & 3.77 & $48.844(3)$ & $7.8050(5)$ & $16126(3)$ & N/A & N/A \\
CuPyr-II & 4.20 & $48.546(4)$ & $7.8242(5)$ & $15969(3)$ & N/A & N/A \\
CuPyr-II & 4.55 & $48.2797(4)$ & $7.8324(6)$ & $15810(3)$ & N/A & N/A \\
CuPyr-II & 4.90 & $48.055(4)$ & $7.8469(6)$ & $15692(3)$ & N/A & N/A
\end{tabular}

Table 5.3 - Unit cell dimensions, volume, SQUEEZE output, including the average void volume and number of electrons per pore as a function of pressure using $\mathrm{MeOH}$ as a hydrostatic liquid, collected using a laboratory diffractometer and $\mathrm{Mo}-\mathrm{K \alpha}$ radiation.

\begin{tabular}{ccccccc}
\hline Phase & $\begin{array}{c}\text { Pressure } \\
(\mathbf{G P a})\end{array}$ & $\mathbf{a} / \mathbf{b}(\boldsymbol{A})$ & $\boldsymbol{c}(\boldsymbol{A})$ & $\begin{array}{c}\text { Volume } \\
\left(\boldsymbol{A}^{3}\right)\end{array}$ & $\begin{array}{c}\text { Average void } \\
\text { volume }\left(\boldsymbol{\AA}^{3}\right)\end{array}$ & $\begin{array}{c}\text { electrons/ } \\
\text { void }\end{array}$ \\
\hline CuPyr-I & 0.34 & $26.620(1)$ & $7.7505(4)$ & $4756.4(5)$ & 416 & 133 \\
CuPyr-I & 0.71 & $26.1591(14)$ & $7.7544(5)$ & $4595.4(5)$ & 392 & 140 \\
CuPyr-I & 1.08 & $25.7864(8)$ & $7.7459(3)$ & $4460.5(3)$ & 365 & 125 \\
CuPyr-I & 1.28 & $25.600(1)$ & $7.7386(4)$ & $4392.2(4)$ & 358 & 134 \\
CuPyr-I & 1.56 & $25.4345(8)$ & $7.7363(3)$ & $4334.2(3)$ & 346 & 113 \\
CuPyr-I & 1.77 & $25.3023(7)$ & $7.7337(3)$ & $4287.8(3)$ & 344 & 115 \\
CuPyr-II & 2.23 & $50.229(3)$ & $7.7339(5)$ & $16898(2)$ & N/A & N/A \\
CuPyr-II & 2.70 & $49.766(4)$ & $7.7685(8)$ & $16662(3)$ & N/A & N/A
\end{tabular}

Table 5.4 - Unit cell dimensions, volume, SQUEEZE output, including the average void volume and number of electrons per pore as a function of pressure using $n$-pentane as a hydrostatic liquid, collected using a laboratory diffractometer and Mo-K $\alpha$ radiation.

\begin{tabular}{ccccccc}
\hline Phase & $\begin{array}{c}\text { Pressure } \\
(\mathbf{G P a})\end{array}$ & $\mathbf{a} / \mathbf{b}(\mathbf{A})$ & $\boldsymbol{c ~ ( \boldsymbol { A } )}$ & $\begin{array}{c}\text { Volume } \\
\left(\mathbf{A}^{3}\right)\end{array}$ & $\begin{array}{c}\text { Average void } \\
\text { volume }\left(\boldsymbol{A}^{3}\right)\end{array}$ & $\begin{array}{c}\text { electrons/ } \\
\text { void }\end{array}$ \\
\hline CuPyr-I & 0.29 & $26.038(3)$ & $7.7843(10)$ & $4570.4(10)$ & 356 & 132 \\
CuPyr-I & 0.77 & $50.919(5)$ & $7.7514(10)$ & $17405(4)$ & N/A & N/A \\
CuPyr-II & 1.61 & $50.017(5)$ & $7.7483(9)$ & $16787(3)$ & N/A & N/A
\end{tabular}


Table $5.5-\mathrm{Cu}-\mathrm{Nl}, \mathrm{Cu}-\mathrm{O} 1$ and $\mathrm{Cu}-\mathrm{O} 2$ in $\mathrm{CuPyr}-\mathrm{I}$ as a function of pressure using FC-70 as a hydrostatic media. $\mathrm{Cu}-\mathrm{N} / \mathrm{O}$ distances are also given for the ambient data set for comparison.

\begin{tabular}{ccccc}
\hline Phase & $\begin{array}{c}\text { Pressure } \\
(\mathbf{G P a})\end{array}$ & Cu-N1 (A) & Cu-01(A) & Cu-O2 (A) \\
\hline CuPyr-I & ambient & $2.332(4)$ & $2.024(3)$ & $1.961(3)$ \\
CuPyr-I & 0.07 & $2.332(5)$ & $2.022(5)$ & $1.960(5)$ \\
CuPyr-I & 0.14 & $2.297(6)$ & $2.040(6)$ & $1.972(5)$ \\
CuPyr-I & 0.33 & $2.198(6)$ & $2.114(6)$ & $1.969(5)$ \\
CuPyr-I & 0.88 & $2.057(9)$ & $2.164(11)$ & $1.978(9)$ \\
CuPyr-I & 1.27 & $2.051(8)$ & $2.200(9)$ & $1.977(7)$ \\
CuPyr-I & 1.56 & $2.046(8)$ & $2.201(10)$ & $1.966(8)$
\end{tabular}

Table 5.6 - Cu-N1, Cu-Ol and Cu-O2 in CuPyr-I as a function of pressure using $\mathrm{MeOH}$ as hydrostatic media. Data is also given for the best data set at $3.34 \mathrm{GPa}$ for the four symmetry independent $\mathrm{Cu}-\mathrm{O} / \mathrm{N}$ bonds in $\mathrm{Cu}-\mathrm{Pyr}-\mathrm{II}$.

\begin{tabular}{ccccc}
\hline Phase & $\begin{array}{c}\text { Pressure } \\
(\text { GPa) }\end{array}$ & Cu-N1 (A) & Cu-O1 (A) & Cu-O2 (A) \\
\hline CuPyr-I & 0.34 & $2.361(5)$ & $1.988(5)$ & $1.963(4)$ \\
CuPyr-I & 0.52 & $2.322(5)$ & $2.007(5)$ & $1.961(4)$ \\
CuPyr-I & 0.71 & $2.277(4)$ & $2.042(4)$ & $1.957(5)$ \\
CuPyr-I & 0.96 & $2.227(6)$ & $2.068(6)$ & $1.966(5)$ \\
CuPyr-I & 1.08 & $2.183(4)$ & $2.113(4)$ & $1.963(3)$ \\
CuPyr-I & 1.28 & $2.140(5)$ & $2.131(6)$ & $1.959(5)$ \\
CuPyr-I & 1.56 & $2.111(3)$ & $2.167(3)$ & $1.959(3)$ \\
CuPyr-I & 1.57 & $2.108(4)$ & $2.161(4)$ & $1.966(4)$ \\
CuPyr-I & 1.77 & $2.092(3)$ & $2.183(3)$ & $1.959(3)$ \\
CuPyr-I & 2.15 & $2.073(5)$ & $2.204(6)$ & $1.952(5)$ \\
CuPyr-II & 3.34 & $2.111(17)$ & $2.147(24)$ & $1.935(19)$ \\
CuPyr-II & 3.34 & $2.108(17)$ & $2.249(23)$ & $1.945(20)$ \\
CuPyr-II & 3.34 & $2.029(16)$ & $2.260(18)$ & $1.893(18)$ \\
CuPyr-II & 3.34 & $2.080(15)$ & $2.308(22)$ & $1.838(18)$
\end{tabular}


Compression of the coordination bonds was not the only distortion to take place in CuPyr-I, with the Cu-octahedra also twisting with respect to the 1-(4pyridyl)butane-1,3-dione linkers on increasing pressure. Twisting of the $\mathrm{Cu}$ octahedra in CuPyr-I with respect to the dione section of the linker could be quantified by measuring both the $\angle \mathrm{N} 1 \mathrm{CulOlC} 2\left(\theta_{1}\right.$ Figure 5.6) and the $\angle \mathrm{N} 1 \mathrm{CulO} 2 \mathrm{C} 4\left(\theta_{2}\right.$ Figure 5.6$)$ torsion angles. In $\mathrm{MeOH} \angle \mathrm{NlCulOlC} 2$ gradually decreases by $12.2^{\circ}$ while $\angle \mathrm{N} 1 \mathrm{CulO} 2 \mathrm{C} 4$ increases sharply initially before plateauing to give a total change of $7.3^{\circ}$ to $2.15 \mathrm{GPa}$ (Table 5.7). In FC-70, $\angle \mathrm{N} 1 \mathrm{CulO1C} 2$ and $\angle \mathrm{N} 1 \mathrm{Cu} 1 \mathrm{O} 2 \mathrm{C} 4$ decrease and increase by $5.4^{\circ}$ and $2.8^{\circ}$ respectively to $1.56 \mathrm{GPa}$ (Table 5.8). On increasing pressure to $1.57 \mathrm{GPa}$ using $\mathrm{MeOH}$, a difference of $\approx 5$ degrees for both angles were observed compared to FC-70 at $1.56 \mathrm{GPa}$.
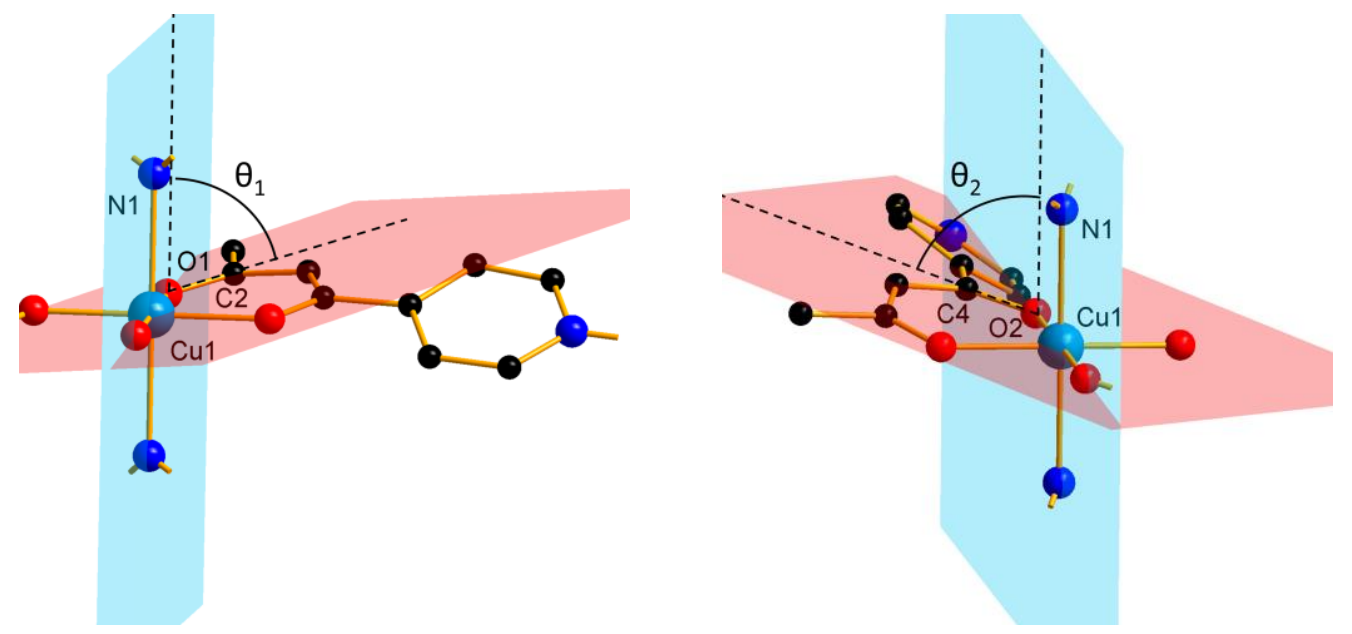

Figure 5.6 - Ball and stick model of CuPyr-I with planes showing the $\angle \mathrm{NlCulOlC} 2\left(\theta_{1}\right)$ and $\angle \mathrm{NlCulO} 2 \mathrm{C} 4\left(\theta_{2}\right)$ torsion angles.

Twisting about the octahedra allows compression of the channels to take place in a 'screw' like fashion and has been observed in other porous materials with channel structures. ${ }^{29}$ The overall effect is to reduce the pore volume, and decrease the size of the channels (Table 5.2, Table 5.3). Using $\mathrm{MeOH}$ as a hydrostatic medium therefore appears to reduce this effect. 
Table $5.7-\angle \mathrm{NlCulO1C2}$ and $\angle \mathrm{NlCulO} 2 \mathrm{C} 4$ torsion angles as a function of pressure using $\mathrm{MeOH}$ as a hydrostatic media.

\begin{tabular}{|c|c|c|}
\hline $\begin{array}{l}\text { Pressure } \\
\text { (GPa) }\end{array}$ & $\angle \mathrm{N} 1 \mathrm{Cu} 101 \mathrm{C2}\left({ }^{\circ}\right)$ & $\angle \mathrm{N} 1 \mathrm{Cu} 102 \mathrm{C} 4\left(^{\circ}\right)$ \\
\hline Ambient & $76.0(4)$ & $-75.9(4)$ \\
\hline 0.34 & $71.2(5)$ & $-69.4(5)$ \\
\hline 0.52 & $69.8(5)$ & $-70.0(5)$ \\
\hline 0.71 & $68.6(5)$ & $-67.9(5)$ \\
\hline 0.96 & $67.7(7)$ & $-69.2(7)$ \\
\hline 1.08 & $67.3(4)$ & $-68.9(4)$ \\
\hline 1.28 & $66.8(6)$ & $-67.5(6)$ \\
\hline 1.56 & $65.4(3)$ & $-69.0(4)$ \\
\hline 1.57 & $65.4(5)$ & $-68.8(5)$ \\
\hline 1.77 & $64.5(4)$ & $-69.1(4)$ \\
\hline 2.15 & $63.8(6)$ & $-68.6(6)$ \\
\hline
\end{tabular}

Table $5.8-\angle \mathrm{NlCulOlC} 2$ and $\angle \mathrm{NlCulO} 2 \mathrm{C} 4$ torsion angles as a function of pressure using FC-70 as a hydrostatic media.

\begin{tabular}{ccc}
\hline $\begin{array}{c}\text { Pressure } \\
(\mathrm{GPa})\end{array}$ & $\angle \mathrm{N} 1 \mathrm{Cu} 101 \mathrm{C2}\left({ }^{\circ}\right)$ & $\angle \mathrm{N} 1 \mathrm{Cu} 102 \mathrm{C4}\left({ }^{\circ}\right)$ \\
\hline Ambient & $76.0(4)$ & $-75.9(4)$ \\
0.07 & $75.8(6)$ & $-74.8(6)$ \\
0.14 & $74.8(6)$ & $-75.1(6)$ \\
0.33 & $74.6(6)$ & $-74.3(6)$ \\
0.88 & $72.2(12)$ & $-76.1(11)$ \\
1.27 & $71.6(10)$ & $-73.8(9)$ \\
1.56 & $70.6(11)$ & $-73.1(10)$
\end{tabular}

Unfortunately, it was not possible to determine the pressure dependence in other longer-chain alcohols, including ethanol (EtOH) and isopropanol (IPA). Upon loading a crystal in EtOH the crystal rapidly started to split. This continued over only a few minutes until the crystal had fully disintegrated making the collection of high pressure data impossible. On loading a separate sample in IPA, similar behaviour was observed, with the crystal losing colour, eventually becoming amorphous over the course of 2-3 hours (Figure 5.7). We believe this is a result of both $\mathrm{EtOH}$ and IPA potentially acting as reducing agents, as indicated by the loss in colour of CuPyr-I on exposure to these media. 

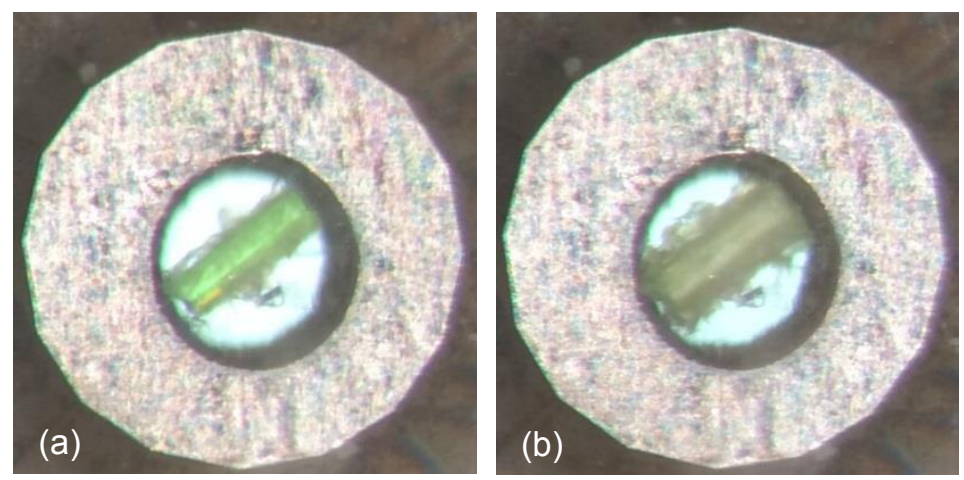

Figure 5.7 - Confocal microscopy images of a loaded diamond anvil cell (DAC) loaded with CuPyr-I using IPA as a hydrostatic media after initial loading (a) and after 12 hours (b).

A compression study was performed using $n$-pentane, the lightest alkane that is a liquid at ambient temperature, as a hydrostatic medium to $1.61 \mathrm{GPa}$. The data only allowed unit cell parameters to be measured and from these $n$-pentane was found to directly compress CuPyr-I (7.97\% reduction in corrected unit cell volume, Table 5.4). On increasing pressure from 0.29 to $0.77 \mathrm{GPa}$, CuPyr-I underwent a phase transition to a CuPyr-II. Again calculated precession images were used to identify the transition, Figure 5.8 .

This transition happens at a much lower pressure than found in $\mathrm{MeOH}$ (2.15 GPa) and we speculated that this difference in pressure is caused by the $n$-pentane entering the channels at a lower pressure than $\mathrm{MeOH}$ due to the hydrophobic nature of the channels. This can be overcome by $\mathrm{MeOH}$ but not until substantially

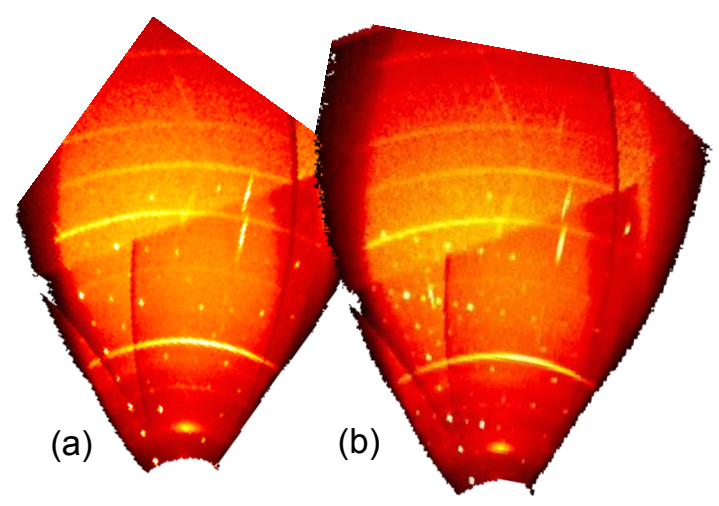

Figure 5.8 - Precession image of the $h k 0$ plane at (a) $0.29 \mathrm{GPa}$, and (b) $0.77 \mathrm{GPa}$ using $n$-pentane as a hydrostatic media. 
higher pressures, an affect which has been seen in other MOFs that contain hydrophobic pores. ${ }^{30}$ However with both a significant reduction in data quality associated with the transition to $\mathrm{CuPyr}-\mathrm{II}$, and a lack of high resolution structural data, it is difficult to resolve or model such behaviour crystallographically.

Compression of the JT axis in both $\mathrm{MeOH}$ and FC-70 caused the crystals to change colour, from light green to dark red on increasing pressure (Figure 5.9). This piezochromic behaviour is consistent with a suppression of the JT axis causing a red-shift, and is fully reversible in both $\mathrm{MeOH}$ and FC-70.
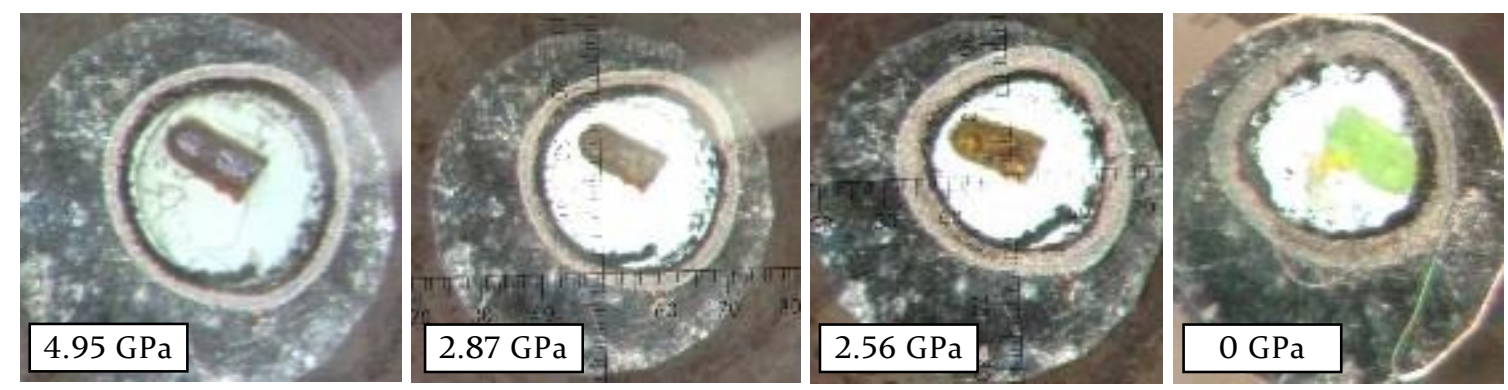

Figure 5.9 - Confocal microscopy images of CuPyr-I on decreasing the pressure in $\mathrm{MeOH}$. Pressures are shown below

\subsubsection{Void content as a function of pressure}

To ascertain the origin of this hydrostatic medium-induced dependence in the JT switching pressure and unit cell compressibility, the pore size and content were monitored as a function of pressure. The pore volume and electron density were calculated and modelled respectively using the SQUEEZE algorithm within PLATON (Table 5.1-5.4). ${ }^{31}$ CuPyr-I under ambient pressure conditions has three symmetry equivalent channels per unit cell with a total volume of $\approx 1152 \AA^{3}$ containing ether trapped in the pores during the synthesis of the framework, confirmed by TGA analysis and SQUEEZE content calculated under ambient pressure and temperature (Table 5.1, Figure 5.10). TGA showed an initial loss of $2.52 \%$ at $140{ }^{\circ} \mathrm{C}$, attributed to the loss of diethyl ether present from the synthesis, and this is followed by the decomposition of CuPyr-I at $220{ }^{\circ} \mathrm{C}$. The entrapped ether could not be modelled atomistically under ambient temperature and temperature conditions. Any attempt to remove the solvent via heating under vacuum also proved unsuccessful, resulting in amorphisation of the crystals. 


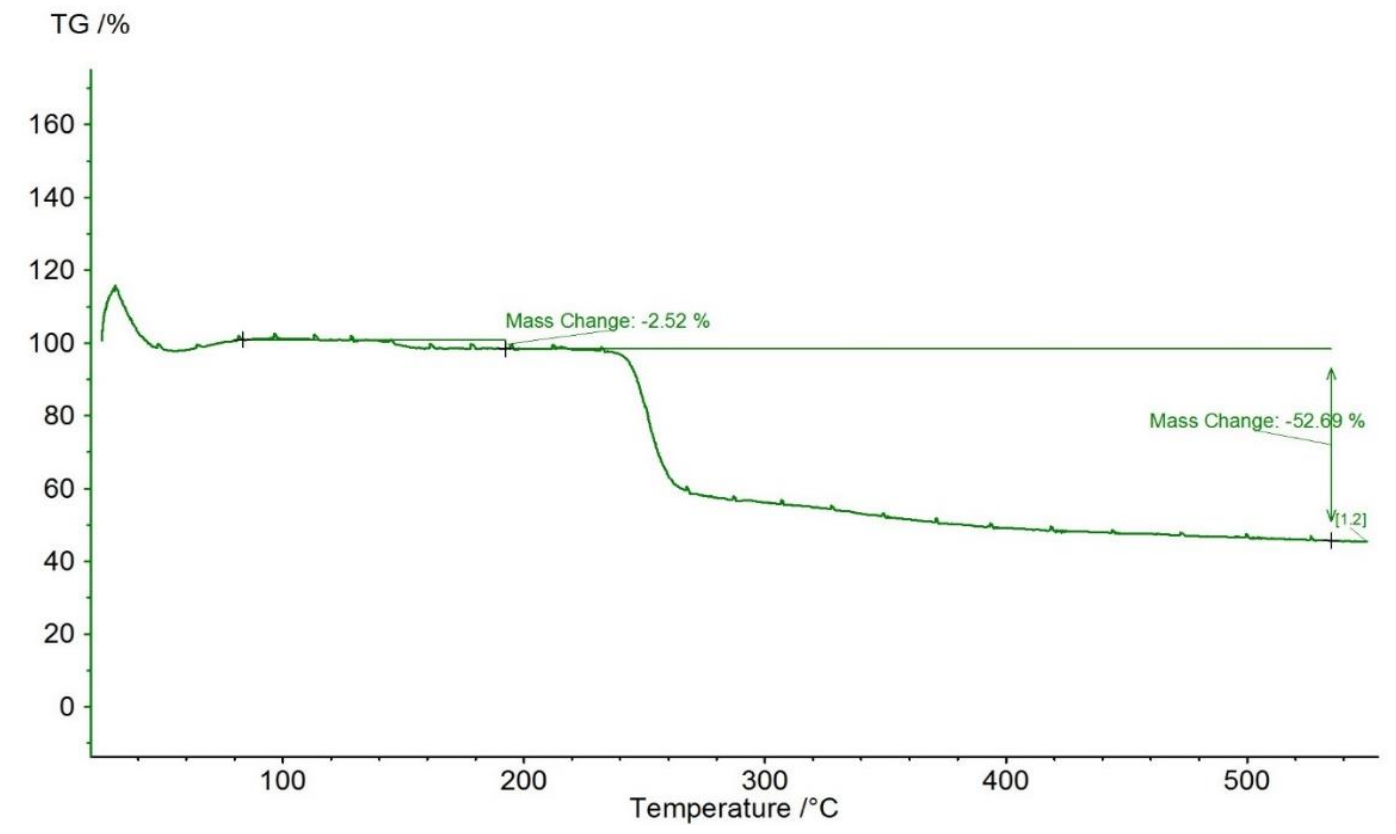

Figure 5.10 - TGA trace of CuPyr-I from room temperature to $550{ }^{\circ} \mathrm{C}$ with a heating rate of $10^{\circ} \mathrm{C} / \mathrm{min}$ under an atmosphere of nitrogen.

On surrounding the crystal with FC-70, no ingress of the hydrostatic liquid into the pores takes place, with the pore content remaining almost constant until 0.88 GPa (Table S1). On increasing pressure further to $1.56 \mathrm{GPa}$, an increase in the calculated electron density was observed (23\%), though the data here were of depreciating quality and less reliable.

Using $\mathrm{MeOH}$ as a hydrostatic medium there was an initial increase in pore volume and electron density in the channels by $4.5 \%$ and $54 \%$ respectively on increasing pressure to $0.52 \mathrm{GPa}$ as the $\mathrm{MeOH}$ entered the pores. On increasing pressure further to $0.96 \mathrm{GPa}$, and then to $2.15 \mathrm{GPa}$, a general reduction in pore content takes place, Table 5.2 \& Table 5.3. This behaviour has been observed in other MOFs, including MOF-5 ${ }^{32}$ and HKUST-1 ${ }^{33}$, and is typical for the ingress of hydrostatic media into the pores, although the exact composition is unknown due to the initial ether content, which could exchange with the $\mathrm{MeOH}$ hydrostatic media. ${ }^{32-34}$

The ingress of $\mathrm{MeOH}$ into the pores on initially increasing pressure to $0.52 \mathrm{GPa}$ is also reflected in the twisting of the $\mathrm{Cu}$-octahedra, in-particular the $\angle \mathrm{N} 1 \mathrm{CulO} 2 \mathrm{C} 4$ angle, which increased by $5.8^{\circ}$ in $\mathrm{MeOH}$, then plateaued, Table 5.7. Whereas on 
compression in FC-70, little to no change was observed in the $\angle \mathrm{N} 1 \mathrm{CulO} 2 \mathrm{C} 4$ angle to $1.56 \mathrm{GPa}$, Table 5.8. These angles represent twisting of the dione backbone, which we speculate must interact with the $\mathrm{MeOH}$ molecules which penetrate into the framework.

The ingress of hydrostatic media would also appear to be responsible for the phase transition from CuPyr-I to CuPyr-II, which is observed in both $\mathrm{MeOH}$ and $n$ pentane, but not FC-70. We note that the transition to CuPyr-II in pentane occurs before $0.77 \mathrm{GPa}$, significantly lower than in $\mathrm{MeOH}$ (2.15 GPa). This difference in pressure is probably caused by the greater ease by which $n$-pentane can enter the framework due to the hydrophobic nature of the channels, which can be overcome using $\mathrm{MeOH}$ as a hydrostatic medium, but not until much higher pressures are reached. This type of behaviour would be consistent with other porous materials studied under pressure, including another $\mathrm{Cu}$-based framework STAM-1. ${ }^{30}$

Unfortunately, because the phase transition from CuPyr-I to CuPyr-II resulted in a significant reduction in data quality, it is difficult to postulate the mechanism behind the transition, and the resulting NLC behaviour.

Further work will be carried out computationally in order to elucidate the structural mechanism that gives rise to the PLC followed by NLC. However, it is clear that the uptake of hydrostatic media is responsible, and therefore the NLC behaviour could well be caused by swelling along the pore channels, caused by uptake of more guest molecules. 


\subsubsection{The effect of decreasing temperature on CuPyr-I}

Low temperature single crystal diffraction at ambient pressure was used in an attempt to obtain an atomistic model of the entrapped ether. On cooling from $300 \mathrm{~K}$ to $150 \mathrm{~K}$, short data collections were performed in order to index the crystal (Table 5.10). On cooling below $150 \mathrm{~K}$ in a single crystal (or $170 \mathrm{~K}$ in a polycrystalline powder), CuPyr-I undergoes a phase transition to a previously unobserved phase, CuPyr-III (Figure 5.11, Table 5.9). CuPyr-III crystallises in the triclinic space group $P-1$, though the pore shape and size remains almost identical to CuPyr-I. In CuPyr-III however, ether molecules could be modelled in the pores (Figure 5.11), and we suspect the transition occurs as a result of the solvent freezing in the channels and breaking the three-fold crystallographic symmetry of the parent phase. The solvent would therefore appear to be an integral part of the pore structure.

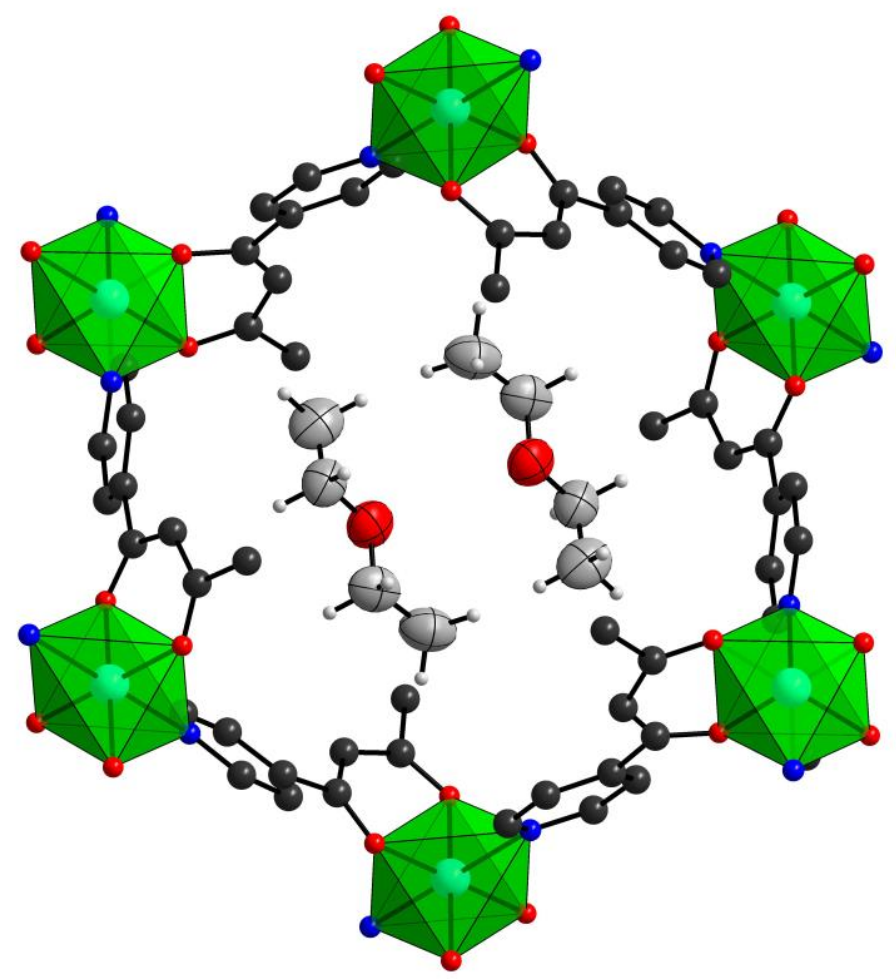

Figure 5.11 - CuPyr-III as viewed along the a-axis direction showing the ordered ether in the pore. Colour scheme is red: Oxygen, blue: Nitrogen, black/grey: Carbon, white: Hydrogen and cyan: Copper. The $\mathrm{Cu}^{2+}$ octahedron are drawn in green with the hydrogens atoms bonded to the framework omitted for clarity. The ether is shown with displacement ellipsoids set to $50 \%$ probability and hydrogen atoms as ball and stick. 
Table 5.9 - Table of structural information for CuPyr-III at 200K.

\begin{tabular}{lll}
\hline Sample & CuPyr-III & \\
Crystal system & Triclinic & \\
Space group & $\mathrm{P}-1$ & \\
Unit cell dimensions & $\mathrm{a}=7.7799(3) \AA$ & $\alpha=116.604(6)^{\circ}$ \\
& $\mathrm{b}=15.1586(9) \AA$ & $\beta=100.395(4)^{\circ}$ \\
& $\mathrm{C}=15.4901(9) \AA$ & $\gamma=98.422(4)^{\circ}$ \\
Volume & $\mathrm{V}=1551.99(15) \AA^{3}$ & \\
Temperature & $120 \mathrm{~K}$ & \\
\hline
\end{tabular}

Table 5.10 - Unit cell dimensions of CyPyr-I on cooling from 300 to $150 \mathrm{~K}$ from singlecrystal diffraction data.

\begin{tabular}{cccccccc}
\hline \multirow{2}{*}{ Temperature (K) } & $\boldsymbol{a}(\boldsymbol{A})$ & $\boldsymbol{b}(\boldsymbol{A})$ & $\boldsymbol{c}(\boldsymbol{A})$ & $\boldsymbol{\alpha}$ & $\boldsymbol{\beta}$ & $\boldsymbol{V}$ & Volume $\left(\boldsymbol{A}^{3}\right)$ \\
\hline 300 & $26.56(1)$ & $26.56(1)$ & $7.708(3)$ & 90 & 90 & 120 & $4709(5)$ \\
250 & $26.48(1)$ & $26.48(1)$ & $7.721(3)$ & 90 & 90 & 120 & $4688(5)$ \\
200 & $26.42(1)$ & $26.42(1)$ & $7.731(3)$ & 90 & 90 & 120 & $4673(5)$ \\
150 & $26.25(2)$ & $26.25(2)$ & $7.723(7)$ & 90 & 90 & 120 & $4609(11)$
\end{tabular}




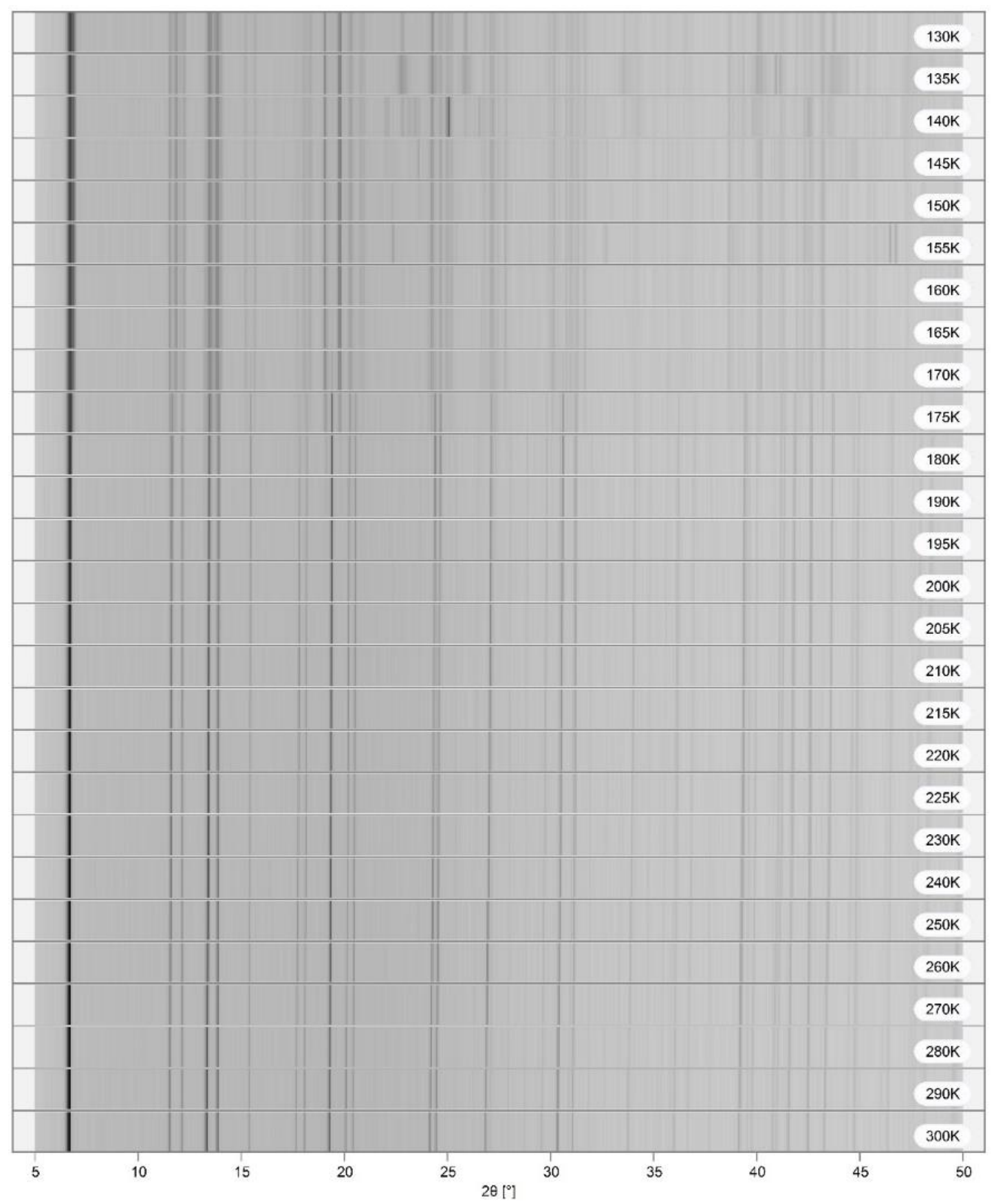

Figure 5.12 - Variable temperature powder diffraction data on CuPyr-I. Note the phase transition from CuPyr-I to CuPyr-III on cooling from 175 to $170 \mathrm{~K}$ respectively. 


\subsection{Conclusions}

In conclusion, we have presented both high-pressure compression and a low temperature diffraction study on a newly synthesised Cu-based framework bis[1(4-pyridyl)butane-1,3-dione]copper(II), CuPyr-I, compressed in FC-70 to $1.56 \mathrm{GPa}$, $\mathrm{MeOH}$ to $4.90 \mathrm{GPa}$, and cooled to $120 \mathrm{~K}$ under ambient pressure conditions. In both FC-70 and $\mathrm{MeOH}$ the JT axis, which extends along the $\mathrm{Cu}-\mathrm{N}$ pyridyl bond, compresses steadily and then switches to lie along one of the $\mathrm{Cu}-\mathrm{O}$ dione bonds. On increasing pressure in $\mathrm{MeOH}, \mathrm{MeOH}$ molecules enter the pores, causing the JT switching pressure to increase from $0.57 \mathrm{GPa}$ in FC-70, to $1.28 \mathrm{GPa}$ in $\mathrm{MeOH}$, causing a greater degree of distortion to occur within the $\mathrm{Cu}$-octahedra. Suppression of the JT axis is also accompanied by a colour change in the crystals from light green to dark red, consistent with suppression of the JT axis. On increasing pressure above $2.15 \mathrm{GPa}$, CuPyr-I undergoes an isosymmetric phase transition to a previously unobserved phase, which results in a doubling of the $a / b$ axes. On increasing pressure NLC behaviour is observed between 2.15 and 4.90 GPa in CuPyr-II, while on cooling below $150 \mathrm{~K}$, a second phase transition occurs to CuPyr-III, caused by ether (from crystallisation) freezing in the pores. Although each of these phenomena has been reported numerous times in a range of materials, this is to the best of our knowledge the first time high-pressure and low temperature phase transitions, NLC, piezochromic and pressure induced JT switching behaviour have been observed within the same material. We have also reported for the first time a pressure induced JT switch which is hydrostatic medium dependant. 


\subsection{References}

1 Moggach, S. A., Parsons, S. \& Wood, P. A. High-pressure polymorphism in amino acids. Crystallography Reviews 14, 143-184, doi:10.1080/08893110802037945 (2008).

2 Moggach, S. A. \& Parsons, S. in Spectroscopic Properties of Inorganic and Organometallic Compounds: Volume 40 Vol. 40 (eds Jack Yarwood, Richard Douthwaite, \& Simon B. Duckett) 324-354 (The Royal Society of Chemistry, 2009).

3 Ohta, K., Kuwayama, Y., Hirose, K., Shimizu, K. \& Ohishi, Y. Experimental determination of the electrical resistivity of iron at Earth's core conditions. Nature 534, 95-98, doi:10.1038/naturel7957 (2016).

4 Umeyama, D., Horike, S., Tassel, C., Kageyama, H., Higo, Y., Hagi, K., Ogiwara, N. \& Kitagawa, S. Pressure-induced amorphization of a dense coordination polymer and its impact on proton conductivity. APL Materials 2, 124401, doi:10.1063/1.4898806 (2014).

5 McKellar, S. C., Sotelo, J., Mowat, J. P. S., Wright, P. A. \& Moggach, S. A. Perfluorocarbon liquid under pressure: a medium for gas delivery. Crystengcomm 18, 1273-1276, doi:10.1039/C5CE01989C (2016).

6 Johnston, B. F., Marshall, W. G., Parsons, S., Urquhart, A. J. \& Oswald, I. D. H. Investigation of Acrylic Acid at High Pressure Using Neutron Diffraction. The Journal of Physical Chemistry B 118, 4044-4051, doi:10.1021/jp502095n (2014).

7 Kiselev, V. D. High-Pressure Influence on the Rate of Diels-Alder Cycloaddition Reactions of Maleic Anhydride with Some Dienes. International Journal of Chemical Kinetics 45, 613-622, doi:10.1002/kin.20800 (2013).

8 McKellar, S. C. \& Moggach, S. A. Structural studies of metal-organic frameworks under high pressure. Acta Crystallographica Section B 71, 587607, doi:10.1107/S2052520615018168 (2015).

9 Sotelo, J., Woodall, C. H., Allan, D. R., Gregoryanz, E., Howie, R. T., Kamenev, K. V., Probert, M. R., Wright, P. A. \& Moggach, S. A. Locating Gases in Porous Materials: Cryogenic Loading of Fuel-Related Gases Into a 
Sc-based Metal-Organic Framework under Extreme Pressures. Angewandte Chemie International Edition 54, 13332-13336, doi:10.1002/anie.201506250 (2015).

10 Cairns, A. B. \& Goodwin, A. L. Negative linear compressibility. Physical Chemistry Chemical Physics 17, 20449-20465, doi:10.1039/C5CP00442J (2015).

11 Parois, P., Moggach, S. A., Sanchez-Benitez, J., Kamenev, K. V., Lennie, A. R., Warren, J. E., Brechin, E. K., Parsons, S. \& Murrie, M. Pressure-induced Jahn-Teller switching in a Mn-12 nanomagnet. Chemical Communications 46, 1881-1883, doi:10.1039/b923962f (2010).

12 Prescimone, A., Morien, C., Allan, D., Schlueter, J. A., Tozer, S. W., Manson, J. L., Parsons, S., Brechin, E. K. \& Hill, S. Pressure-Driven Orbital Reorientations and Coordination-Sphere Reconstructions in [CuF2(H2O)2(pyz)]. Angewandte Chemie International Edition 51, 74907494, doi:10.1002/anie.201202367 (2012).

13 SAINT v. 8.34A (Bruker AXS Inc., Madison, Wisconsin, USA, 2014).

14 SADABS v. 2008-1 ed. (Bruker AXS Inc., Madison, Wisconsin, USA, 2014/15).

15 Merrill, L. \& Bassett, W. A. Miniature diamond anvil pressure cell for single-crystal X-ray-diffraction studies. Review of Scientific Instruments $\mathbf{4 5}$, 290-294, doi:10.1063/1.1686607 (1974).

16 Moggach, S. A., Allan, D. R., Parsons, S. \& Warren, J. E. Incorporation of a new design of backing seat and anvil in a Merrill-Bassett diamond anvil cell. Journal of Applied Crystallography 41, 249-251, doi:10.1107/S0021889808000514 (2008).

17 Piermarini, G. J., Block, S., Barnett, J. D. \& Forman, R. A. Calibration of pressure-dependence of $\mathrm{Rl}$ ruby fluorescence line to 195 kbar. Journal of Applied Physics 46, 2774-2780, doi:10.1063/1.321957 (1975).

18 Dawson, A., Allan, D. R., Parsons, S. \& Ruf, M. Use of a CCD diffractometer in crystal structure determinations at high pressure. Journal of Applied Crystallography 37, 410-416, doi:10.1107/S0021889804007149 (2004).

19 APEX2 v. v2014.11-0 (Bruker AXS Inc., Madison, Wisconsin, USA, 2014). 
20 Sheldrick, G. SHELXT - Integrated space-group and crystal-structure determination. Acta Crystallographica Section A 71, 3-8, doi:10.1107/S2053273314026370 (2015).

21 Betteridge, P. W., Carruthers, J. R., Cooper, R. I., Prout, K. \& Watkin, D. J. CRYSTALS version 12: software for guided crystal structure analysis. Journal of Applied Crystallography 36, 1487-1487, doi:10.1107/s0021889803021800 (2003).

22 Ortiz, A. U., Boutin, A., Fuchs, A. H. \& Coudert, F.-X. Investigating the Pressure-Induced Amorphization of Zeolitic Imidazolate Framework ZIF8: Mechanical Instability Due to Shear Mode Softening. The Journal of Physical Chemistry Letters 4, 1861-1865, doi:10.1021/jz400880p (2013).

23 Bennett, T. D., Saines, P. J., Keen, D. A., Tan, J.-C. \& Cheetham, A. K. BallMilling-Induced Amorphization of Zeolitic Imidazolate Frameworks (ZIFs) for the Irreversible Trapping of Iodine. Chemistry - A European Journal 19, 7049-7055, doi:10.1002/chem.201300216 (2013).

24 Lanza, A., Fiolka, C., Fisch, M., Casati, N., Skoulatos, M., Ruegg, C., Kramer, K. W. \& Macchi, P. New magnetic frameworks of [(CuF2(H2O)2)x(pyz)]. Chem. Commun. 50, 14504-14507, doi:10.1039/C4CC06696K (2014).

25 Qiao, Y., Wang, K., Yuan, H., Yang, K. \& Zou, B. Negative Linear Compressibility in Organic Mineral Ammonium Oxalate Monohydrate with Hydrogen Bonding Wine-Rack Motifs. The Journal of Physical Chemistry Letters 6, 2755-2760, doi:10.1021/acs.jpclett.5b01129 (2015).

26 Yeung, H. H. M., Kilmurray, R., Hobday, C. L., McKellar, S. C., Cheetham, A. K., Allan, D. R. \& Moggach, S. A. Hidden negative linear compressibility in lithium 1-tartrate. Physical Chemistry Chemical Physics 19, 3544-3549, doi:10.1039/C6CP08690J (2017).

27 Cliffe, M. J. \& Goodwin, A. L. PASCal: a principal axis strain calculator for thermal expansion and compressibility determination. Journal of Applied Crystallography 45, 1321-1329, doi:10.1107/S0021889812043026 (2012).

28 Li, W., Probert, M. R., Kosa, M., Bennett, T. D., Thirumurugan, A., Burwood, R. P., Parinello, M., Howard, J. A. K. \& Cheetham, A. K. Negative 
Linear Compressibility of a Metal-Organic Framework. Journal of the American Chemical Society 134, 11940-11943, doi:10.1021/ja305196u (2012).

29 Moggach, S. A., Gorbitz, C. H. \& Warren, J. E. The effect of pressure on the porous peptide l-alanyl-l-valine. Crystengcomm 12, 2322-2324, doi:10.1039/C000942C (2010).

30 McKellar, S. C., Graham, A. J., Allan, D. R., Mohideen, M. I. H., Morris, R. E. \& Moggach, S. A. The effect of pressure on the post-synthetic modification of a nanoporous metal-organic framework. Nanoscale 6, 41634173, doi:10.1039/c3nr0416la (2014).

31 Spek, A. L. PLATON SQUEEZE: a tool for the calculation of the disordered solvent contribution to the calculated structure factors. Acta Crystallographica Section C 71, 9-18, doi:10.1107/s2053229614024929 (2015).

32 Graham, A. J., Allan, D. R., Muszkiewicz, A., Morrison, C. A. \& Moggach, S. A. The Effect of High Pressure on MOF-5: Guest-Induced Modification of Pore Size and Content at High Pressure. Angewandte Chemie International Edition 50, 11138-11141, doi:10.1002/anie.201104285 (2011).

33 Graham, A. J., Tan, J.-C., Allan, D. R. \& Moggach, S. A. The effect of pressure on Cu-btc: framework compression vs. guest inclusion. Chemical Communications 48, 1535-1537, doi:10.1039/C1CC16045A (2012).

34 Graham, A. J., Banu, A.-M., Dueren, T., Greenaway, A., McKellar, S. C., Mowat, J. P. S., Ward, K., Wright, P. A. \& Moggach, S. A. Stabilization of Scandium Terephthalate MOFs against Reversible Amorphization and Structural Phase Transition by Guest Uptake at Extreme Pressure. Journal of the American Chemical Society 136, 8606-8613, doi:10.1021/ja411934f (2014). 


\section{- Chapter 6 -}

\section{The Effect of Pressure,}

\section{Temperature, and Chemical}

Stability of Phthalocyanine

Porous Molecular Crystals 


\subsection{Introduction}

\subsubsection{Porous Molecular Crystals}

Materials such as Metal-Organic Frameworks (MOFs), ${ }^{1}$ Covalent Organic Frameworks $(\mathrm{COFs})^{2}$ and porous organic polymers ${ }^{3,4}$ form part of an emergent field of porous materials. They are characterised by containing interconnected pores of molecular-sized dimensions and have found uses in a wide range of applications from new catalysts to drug delivery. ${ }^{5-9}$ MOFs and COFs are formed from a network of intramolecular chemical bonds that can give rise to highly ordered crystalline materials.

Another class of porous material are porous molecular crystals (PMCs). These are assembled from discrete molecules between which there are only non-covalent intermolecular interactions. However, most molecular systems tend to crystallise in a dense form, to maximise intermolecular interactions and minimise void space. This is obviously not conducive to the formation of a porous material. ${ }^{10,11}$ One way to overcome this is to have a cavity built into the molecule, referred to as intrinsic porosity. A typical example of this would be porous cage like rigid molecules with windows that allow access to these permanent voids, Figure 6.1a. ${ }^{12-17}$

An alternative to intrinsic porosity is extrinsic porosity where the pore is inbetween neighbouring molecules, Figure 6.1b. ${ }^{18-24}$ Molecules that are unable to pack efficiently can crystallize in a low-density form. This inefficient packing causes void space to form between two or more non-covalently bound molecules and, if interconnected, can lead to porosity (defined as the ability for molecules to penetrate the crystalline material). The intermolecular interactions can be directional (such as hydrogen bonding, $\pi-\pi$ stacking, or $\mathrm{CH} \cdots \pi$ interactions) or contain solely van der Waal interactions.

In all cases, PMCs are formed by simple crystallisation rather than through selfassembly and bond formation, which occurs in framework materials such as MOFs, COFs, and zeolites. This affords PMCs with the distinct advantage of being able to be processed in solution, as they can be dissolved and reassembled without the need to break coordination or covalent bonds, allowing purification via recrystallization. ${ }^{17,21,25,26}$ Solution based techniques, such as solvent evaporation, 
(a)

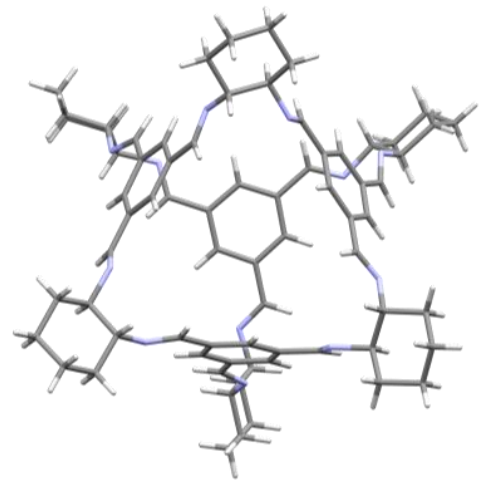

(b)

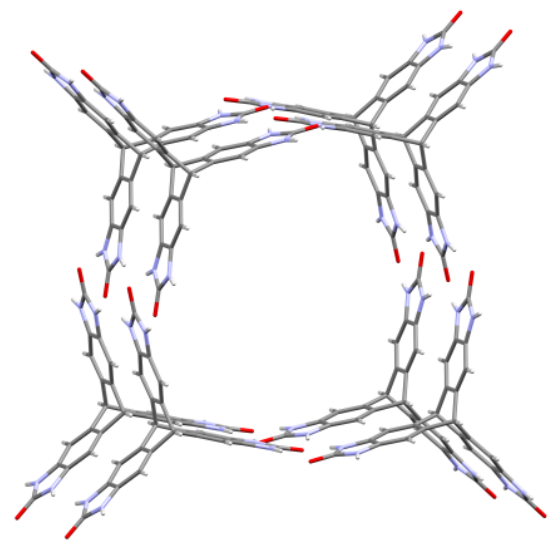

Figure 6.1 - (a) An example of a PMC with intrinsic porosity, 'Cage 3' from Cooper et al., 2009, a tetrahedral organic cage with void space contained within the molecule itself. (b) An example of extrinsic porosity triptycene trisbenzimidazolone (TTBI) from Mastalerz et al., 2012, where the porosity is between molecules.

are commonly used to form high quality single crystals. PMCs are often initially formed with the solvent of crystallisation as an inclusion compound, and to activate the material (i.e. have available pore-space to occupy) the solvent must be removed. For most systems the removal of the inclusion compound results in structural collapse to a denser crystalline or amorphous form, Figure 6.2a,b. ${ }^{27}$ Some PMC's are not stable to the evacuation of the inclusion compound, however, are stable to solvent exchange. This makes them potentially useful for molecular separations, Figure 6.2 c. $^{28-32}$

Structures that allow the full evacuation of the included solvent to form a 'permanent' PMC are still rare, especially when compared to other crystalline porous network materials such as MOFs and COFs, Figure 6.2d. ${ }^{33}$ To test and confirm permanent porosity, the PMC must demonstrate reversible gas adsorption. ${ }^{34}$ The gas used will vary from one system to another. ${ }^{27}$ A few PMCs can now match the high surface areas of conventional porous materials, ${ }^{17,21,35}$ nevertheless, it is questionable whether PMCs possess the chemical and environmental stability for many of the practical applications for which porous organic materials are being proposed. ${ }^{36}$ Indeed, to date, only a few porous molecular crystals have demonstrated stability towards high temperatures, ${ }^{15,37-40}$ strong aqueous acid or base, ${ }^{38,41}$ and, to date, none have been shown to survive extreme pressures. 


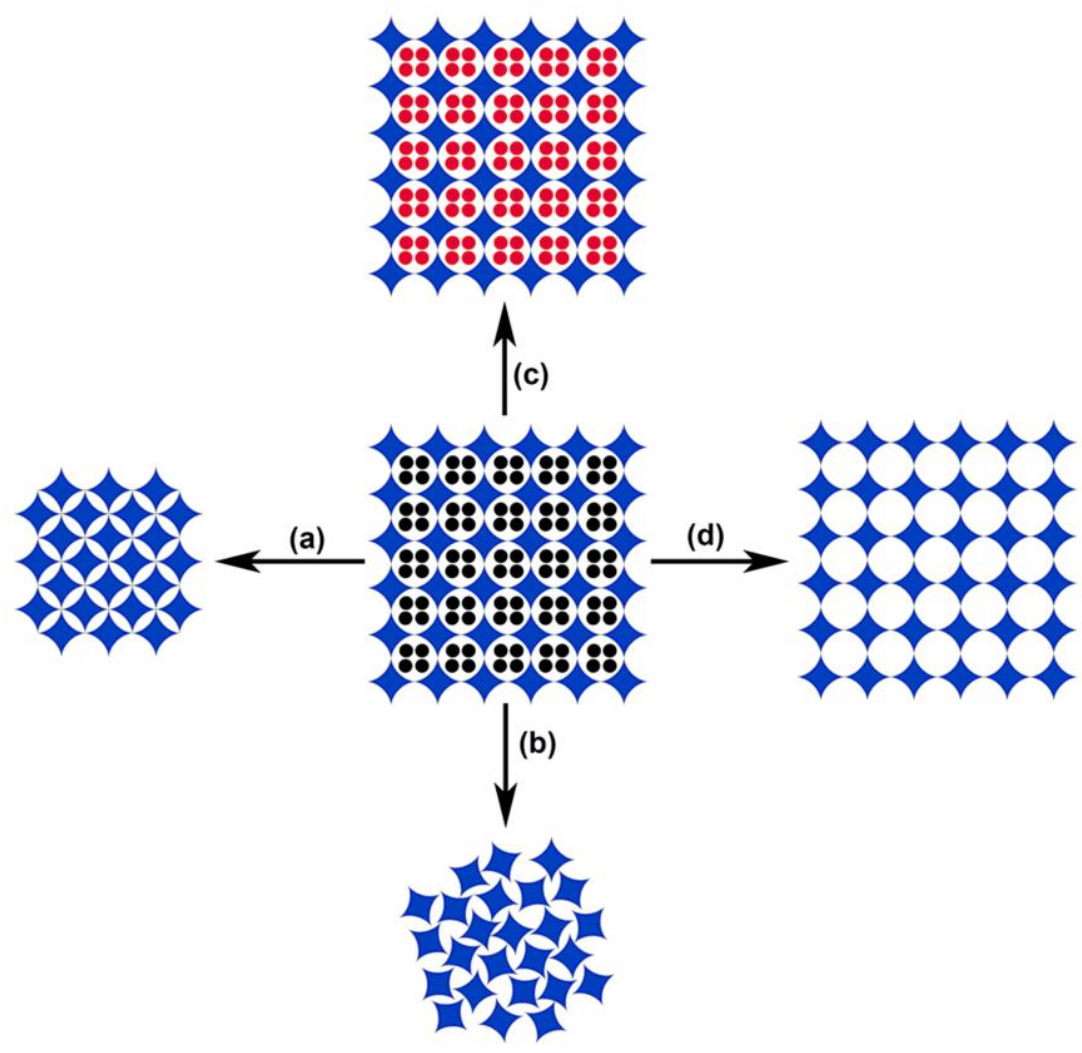

Figure 6.2 - Possible processes involving removal or exchange of included solvent within an inclusion compound: (a) transformation to a non-porous, denser crystal; (b) collapse of crystal structure to a non-porous amorphous solid; (c) exchange of included solvent; and (d) formation of a porous molecular crystal. (Figure adapted from McKeown, 2010)

\subsubsection{Phthalocyanine Porous Molecular Crystals}

The PMCs that are investigated in this chapter are constructed with metal complexes of 2,3,9,10,16,17,23,24-octa(2',6'-di-iso-propylphenoxy)phthalocyanine, (dipPhO) $)_{8}$ PcM, (Figure 6.3) first reported in 2005 by McKeown et al. ${ }^{42}$

These form a clathrate structure that crystallise in the high symmetry cubic space group $P n \overline{3} n$ with a large unit cell $(a \sim 37 \AA)$. The unit cell contains cubic assemblies of six phthalocyanine molecules surrounding very large $\left(8 \mathrm{~nm}^{3}\right)$ solvent filled voids that are interconnected at each corner through channels of $\sim 4 \AA$ in diameter. In addition to this large void there are smaller cavities that lie between the phthalocyanine assemblies, $\sim 12 \AA$ in diameter. 
Isostructural crystal structures occur for most metal complexes of (dipPhO) ${ }_{8} \mathrm{PcM}$ (e.g. $\mathrm{M}=\mathrm{Mg}^{2+}, \mathrm{Al}^{3+}, \mathrm{Ti}^{4+}, \mathrm{Mn}^{2+}, \mathrm{Fe}^{2+}, \mathrm{Co}^{2+}, \mathrm{Zn}^{2+}, \mathrm{Ru}^{2+}, \mathrm{In}^{3+}$ ) as well as the metal free complex (dipPhO) ${ }_{8} \mathrm{PcH}_{2}$. This packing is also not effected by variation in size, shape, type and number of axial ligands attached to the metal cation. ${ }^{43}$ The original solvent of recrystallisation within the void and cavity can be rapidly and

(a)
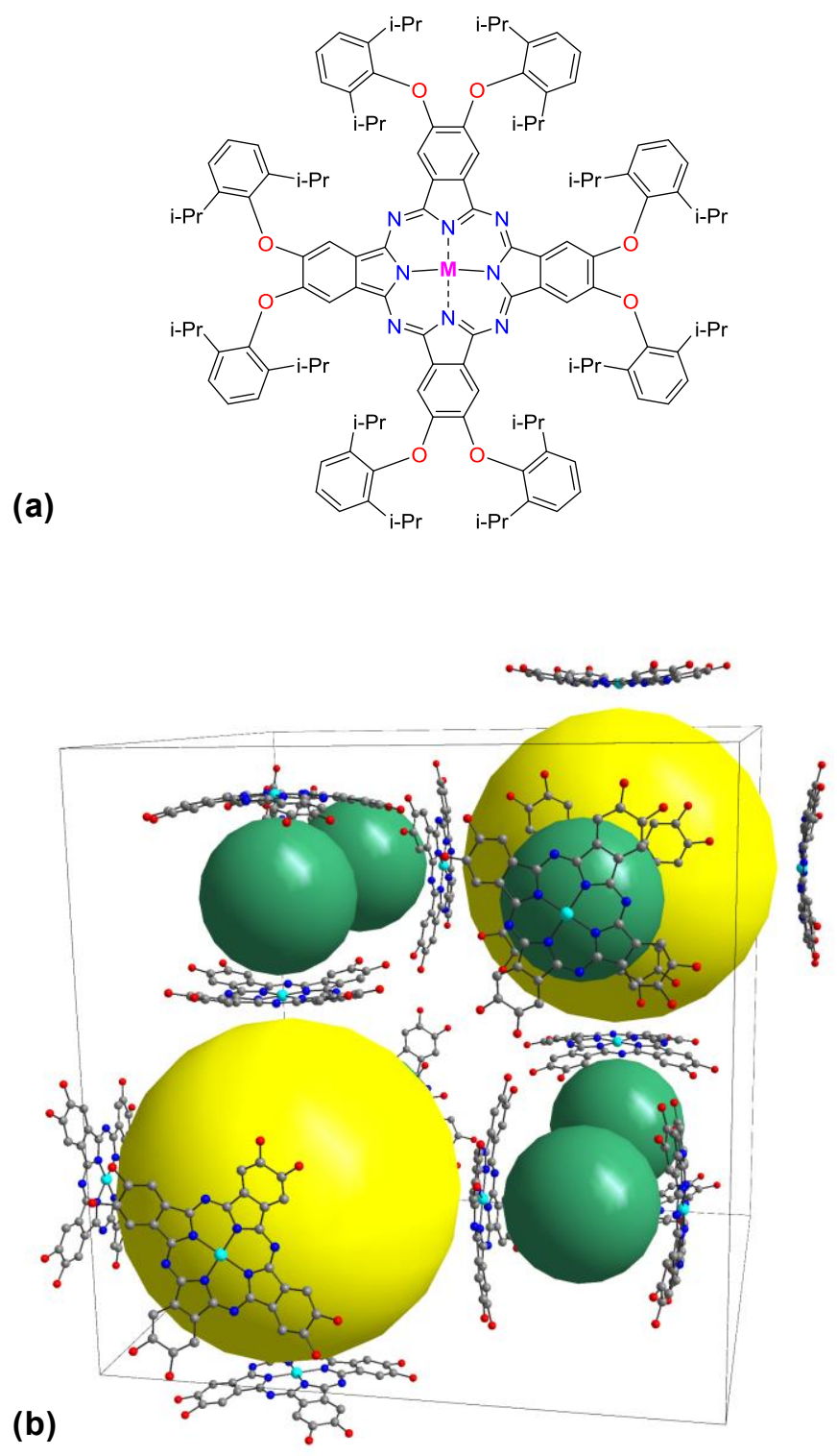

Figure 6.3 - (a) General molecular structure of (dipPhO) ${ }_{8} \mathrm{PcM}$. (b) A perspective representation of the crystal structure of (dipPhO) ${ }_{8} \mathrm{PcZn}$ with the substituent groups and hydrogens removed for clarity. The yellow and green spheres represent the voids and cavities respectively. The volume of the spheres are set equal to the solvent accessible void volume as calculated by SQUEEZE, probe radius $1.2 \AA$. 

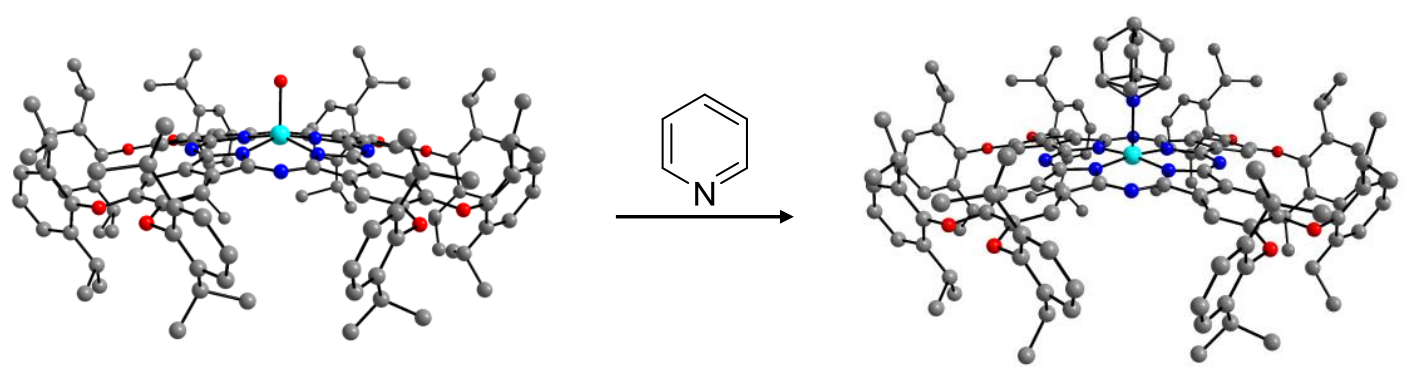

Figure 6.4 - In-situ single-crystal to single-crystal, ligand exchange of water for pyridine within the large void within crystals of (dipPhO) $)_{8} \mathbf{P c Z n}$. The hydrogens are removed for clarity.

reversibly exchanged with other solvents as can the axial ligands on the metal cation, Figure 6.4. ${ }^{44}$ However, as is commonly encountered in molecular crystals containing very large solvent-filled voids, structural collapse occurs rapidly on removal of the solvate.

Previous work has shown that both axial positions on the metal are accessible for coordination, and by using a suitably sized bidentate ligand (e.g. 4,4'-bipyridyl, bipy; 1,4-phenylenediisocyanide, PDIC) the cavity between two metal cations could be bridged joining two phthalocyanine molecules (referred to as 'wall ties'), Figure 6.5. It is important to note that these molecular wall-ties bridge two
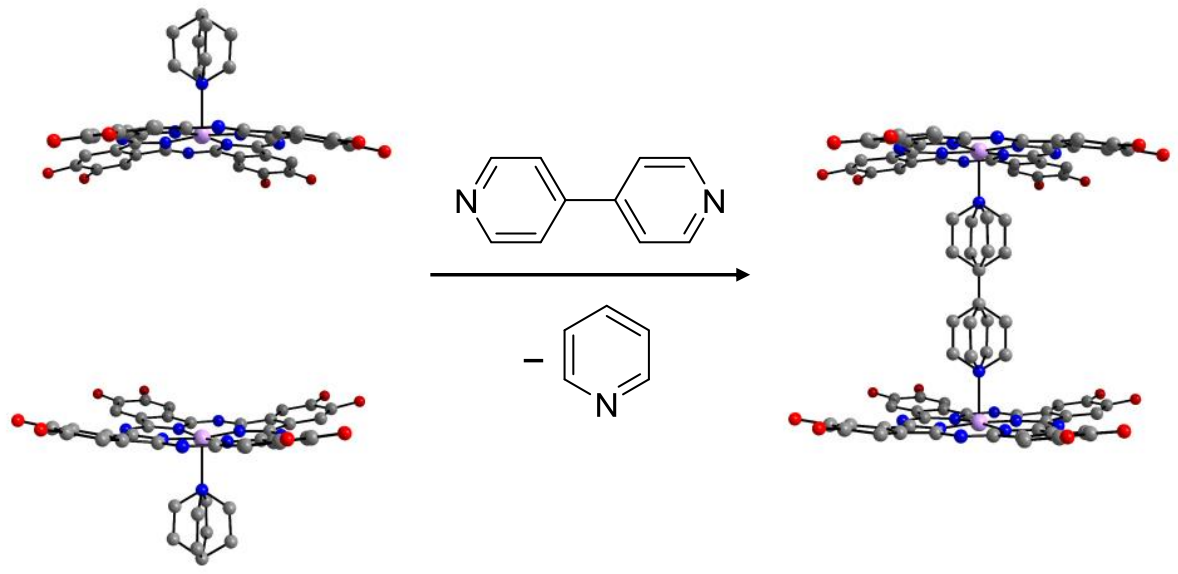

Figure 6.5 - In-situ single crystal to single-crystal transformation with bipy ligand coordinating across the cavities of PNC[Co-vPy] to act as a wall-tie resulting in the formation of PUNC[Co-cbipy-Co]. The substituents and hydrogens are removed for clarity. 
phthalocyanines to form a dimeric complex rather than an extended framework, therefore these are still molecular crystal structures. The wall-ties provided enough stability to allow the total removal of the included solvent resulting in permanently porous Phthalocyanine Unsolvated Nanoporous Crystals (or PUNCs), as demonstrated by nitrogen adsorption. ${ }^{43}$ However, these wall-tie ligands are lost at moderate temperatures and under acidic conditions. This lack of stability could limit the potential application of these PUNCs, for example, in heterogenous catalysis. $^{45}$

Here we present the exceptional stability of a permanently porous Phthalocyanine Nanoporous Crystal (PNC) prepared by the simple co-crystallisation of $(\text { dipPhO })_{8}$ PcCo and a fullerene $\left(\mathrm{C}_{60}\right.$ or $\left.\mathrm{C}_{70}\right)$. These crystals retain their porous structure on evacuation of solvent; heating; during prolonged immersion in boiling aqueous acid, base, and in water; and at extreme pressures, where we report the first high pressure study of a PMC. The stabilizing fullerene is captured in the cavity between two phthalocyanines in a ball and socket arrangement and affords remarkable resilience to the application of external hydrostatic pressure. 


\subsubsection{A Note on Nomenclature}

Porous Molecular Crystal, PMC, refers to all porous molecular systems and so is a general term. Phthalocyanine Nanoporous Crystal, PNC, here will refer to solvated materials with the cubic (dipPhO) $)_{8} \mathbf{P c M}$ structure whether they are permanently porous or not. Phthalocyanine Unsolvated Nanoporous Crystal, PUNC, is a PNC that has been evacuated of solvent and therefore is permanently porous.

The naming scheme for these PNC's was set out by Bezzu et al. 2013, Figure 6.6. ${ }^{44}$ This allows the shorthand recognition of the construction on the PNCs and whether a ligand is facing into the large void or the smaller cavity.
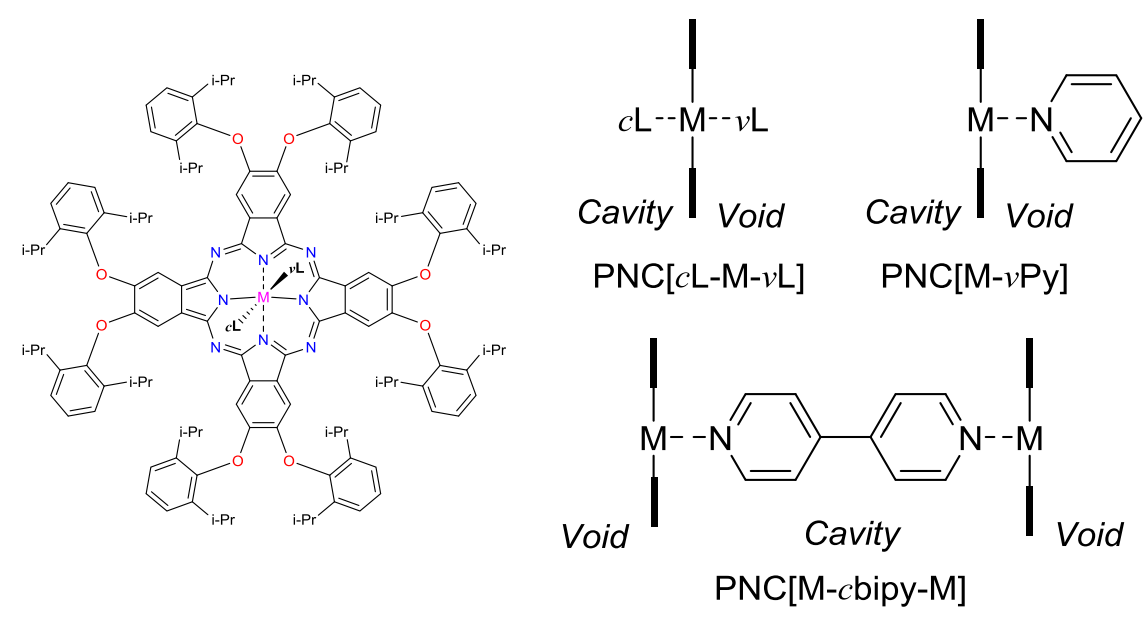

Figure 6.6 - The outlined naming scheme for the PNCs showing the structure of (dipPhO $)_{8}$ PcM along with two example PNCs, PNC[M-vPy and PNC[M-cbipy-M] Adapted from Bezzu et al. 2013. 


\subsection{Experimental}

Note: Synthesis, purity, chemical stability, and volumetric $N_{2}$ sorption studies of (dipPhO $)_{8} P c M,\left(M=H_{2}\right.$, Co) were carried out by Dr C. Grazia Bezzu and Mr Luke Burt from the University of Edinburgh. Variable temperature powder diffraction measurements were conducted by Dr Stephen A. Moggach from the University of Edinburgh. These are included here for completeness.

\subsubsection{Equipment}

Melting points were recorded using a Gallenkamp melting point apparatus and are uncorrected. Infra-red spectra were recorded in the range $4000-600 \mathrm{~cm}^{-1}$ using a Jasco FTIR-660 plus spectrometer. Elemental analysis was carried out with a Carlo Erba EAllolor a CE 440 elemental analyser. UV/vis absorption spectra were recorded in the range 200-800 $\mathrm{nm}$ using a Jasco V-570 UV/vis/NIR spectrophotometer. Volumetric $\mathrm{N}_{2}$ sorption studies were undertaken at $77 \mathrm{~K}$ using a Beckman Coulter 3100 Surface Area Analyzer (Fullerton, California, USA). ${ }^{1} \mathrm{H}$ NMR spectra were measured in $\mathrm{CDCl}_{3}$ using an Avance Bruker DPX 400 instrument (400 MHz), with ${ }^{13} \mathrm{C}$ NMR spectra recorded at $100 \mathrm{MHz}$ respectively. MALDI-TOF mass spectroscopic analyses were performed with a Waters MALDI Micro MX spectrometer. Thermogravimetric analysis was performed on a TA SDT Q 600 instrument.

\subsubsection{Synthesis}

(dipPhO) $)_{8} \mathbf{P c H}_{2}$. - 2,3,9,10,16,17,23,24-octa(2',6'-di-iso-propylphenoxy)phthalocyanine.

To a solution of 4,5-bis(2,6-di-iso-propylphenoxy)phthalonitrile (0.20 g, 0.42 $\mathrm{mmol})$ in refluxing anhydrous 1-pentanol $(3 \mathrm{ml})$, under nitrogen, an excess of lithium was added. The reaction mixture was refluxed for $5 \mathrm{~h}$ and then cooled and acetic acid added $(1 \mathrm{ml}, 0.1 \mathrm{M})$. The solvent was removed under reduced pressure to give the crude product. Purification by column chromatography on $\mathrm{SiO} 2$, eluting with DCM, gave the title compound as a green solid (0.08 g, 40\%). 
M.p. > $300{ }^{\circ} \mathrm{C}$; IR (film)/cm ${ }^{-1}$ 3576, 2962, 1439, 1328, 1265, 1184, 1093, 1017, 877, 754; UV/vis (DCM): $\lambda_{\max } 702.5,667.5,607,420.5,348.5,297,229.5 \mathrm{~nm} ;{ }^{1} \mathrm{H}$ NMR $\left(500 \mathrm{MHz} ; \mathrm{CDCl}_{3}\right) \delta 8.13(\mathrm{br}, 8 \mathrm{H}), 7.50(\mathrm{~m}, 24 \mathrm{H}, \mathrm{ArH}), 3.37$ (sept, 16H, J = $6.5 \mathrm{~Hz}$, $\mathrm{CH}_{3} \mathrm{CHCH}_{3}$ ), 1.28 (br m, $96 \mathrm{H}, \mathrm{CH}_{3} \mathrm{CHCH}_{3}$ ), -0.84 (s, 2H, NH); ${ }^{13} \mathrm{C}$ NMR (125 MHz; $\left.\mathrm{CDCl}_{3}\right) \delta 151.0,149.1,141.7,126.4,124.7,107.4,27.4$, some aromatic Cs are missing which is commonly encountered in phthalocyanine ${ }^{13} \mathrm{C}$ NMR spectra; MS (MALDITOF): cluster centred at $\mathrm{m} / \mathrm{z}$ 1925.71 $\left(\mathrm{MH}^{+}\right)$; elemental analysis calc (\%) for $\mathrm{C}_{128} \mathrm{H}_{146} \mathrm{~N}_{8} \mathrm{O}_{8}$ : C 79.88, H 7.65, N 5.82, found C 80.04, H 7.70, N 5.82.

(dipPhO) $)_{8} P c C o$ - Cobalt 2,3,9,10,16,17,23,24-octa(2',6'-di-iso-propylphenoxy)phthalocyanine,

A stirred solution of 4,5-bis(2,6-di-iso-propylphenoxy)phthalonitrile (0.50 g, 1.04 $\mathrm{mmol})$ and cobalt(II)acetate $(0.18 \mathrm{~g}, 1.01 \mathrm{mmol})$ in anhydrous NMP (1 ml) was heated at $165^{\circ} \mathrm{C}$ for $20 \mathrm{~h}$ under nitrogen. The reaction mixture was cooled to room temperature and poured into water $(15 \mathrm{ml})$. The crude product was collected by filtration. Purification by column chromatography on $\mathrm{SiO}_{2}$, eluting with hexane$\operatorname{DCM}(4: 6)$, gave the title compound as a green solid (0.22 g, 40\%). M.p. $>300{ }^{\circ} \mathrm{C}$; IR (film)/cm ${ }^{-1}$ 2961, 1612, 1456, 1462, 1413, 1353, 1269, 1186, 1095, 1050, 904, 864, 799, 777, 755, 729; UV/vis (DCM): $\lambda_{\max }$ 673, 608, 404, 331, 302, 229 nm; MS (MALDI-TOF): cluster centred at $\mathrm{m} / \mathrm{z} 1982.055\left(\mathrm{MH}^{+}\right)$; elemental analysis calc (\%) for $\mathrm{C}_{128} \mathrm{H}_{144} \mathrm{~N}_{8} \mathrm{O}_{8} \mathrm{Co}$ : C 77.59, H 7.32, N 5.66 found C 77.68, H 7.52, N 5.52.

Commercially available reagents were purchased from Sigma-Aldrich and used without further purification. Fullerenes C60 98\% purity and C70 99\% purity were used. Unless otherwise stated all reactions were carried out under an inert atmosphere of dry nitrogen gas. Oven-dried glassware was left in a $100{ }^{\circ} \mathrm{C}$ oven for at least 2 hrs and cooled under dry nitrogen flow prior to use. 


\subsubsection{Crystallisations}

PNC $\left[c_{60}-\mathrm{Co}\right]$ and $\mathrm{PNC}\left[\mathrm{cC}_{70}-\mathrm{Co}\right]$ crystals were obtained by slow diffusion of methanol into equimolar toluene solutions of (dipPhO) $)_{8} P c C o$ and $\mathrm{C} 60$ or C70 respectively and then filtered and dried under flow of nitrogen to obtain their relative desolvated crystals $\mathbf{P U N C}\left[\boldsymbol{c C}_{60}-\mathbf{C o}\right]$ and $\mathbf{P U N C}\left[\boldsymbol{c C}_{70}-\mathbf{C o}\right]$. The purity of PNC $\left[\boldsymbol{c C}_{60}-\mathbf{C o}\right]$ crystals was tested by elemental analysis using a Carlo Erba EAllolor a CE 440 elemental analyser: Calc (\%) for $\mathrm{C}_{188} \mathrm{H}_{144} \mathrm{~N}_{8} \mathrm{O}_{8} \mathrm{Co}$ : C 83.56, H 5.37, N 4.15 found C 80.90, H 5.43, N 4.35.

PNC [Co-vPy] crystals were prepared by a slow diffusion of acetone into a $\mathrm{CHCl}_{3}$ solution of (dipPhO) $)_{8} P c C o(0.01 \mathrm{~g}, 0.005 \mathrm{mmol})$, to which had been previously added 2 drops of pyridine. CCDC deposition number 761407, Refcode VUSFEV.

PNC $\left[v(\mathrm{Py})\right.$ 0.6 $\left(\mathrm{H}_{2} \mathrm{O}\right)$ 0.4-Co-cbipy-Co- $\left.v(\mathrm{Py}) 0.6\left(\mathrm{H}_{2} \mathrm{O}\right) 0.4\right]$ crystals were prepared by the addition of an excess of 4,4-bipyridyl to some PNC[Co-vPy] crystals still in contact with the solvent of crystallisation (MeOH/chloroform/Py). CCDC deposition number 761419, Refcode VUSHOH.

PUNC[Co-cbipy-Co] crystals were prepared by exposing crystals PNC $\left[v(\mathrm{Py}) 0.6\left(\mathrm{H}_{2} \mathrm{O}\right) 0.4\right.$-Co-cbipy-Co- $\left.v(\mathrm{Py}) 0.6\left(\mathrm{H}_{2} \mathrm{O}\right) 0.4\right]$ to a stream of dry nitrogen at $20^{\circ} \mathrm{C}$. CCDC deposition number 761420, Refcode VUSHUN.

\subsubsection{Permanent porosity determination}

Volumetric $\mathrm{N}_{2}$ adsorption/desorption for a sample of $\mathbf{P N C}\left[\boldsymbol{c C}_{60}-\mathbf{C o}\right]$, which had been previously outgassed under vacuum at $125^{\circ} \mathrm{C}$ for $2 \mathrm{~h}$, was performed using a Beckman Coulter 3100 Surface Area Analyzer (Fullerton, California, USA) at $77 \mathrm{~K}$.

\subsubsection{Thermal gravimetric analyses (TGA)}

The thermal stabilities of $\mathrm{C} 60$ and the unsolvated crystals of PUNC $\left[\mathbf{c C}_{\mathbf{6 0}}-\mathbf{C o}\right]$, $(\text { dipPhO })_{8}$ PcCo, and PUNC[cbipy-Co] were measured by thermal gravimetric analysis using a TA SDT Q 600 instrument (Figure 6.9). The temperature was ramped by $10{ }^{\circ} \mathrm{C}$ per minute to $1000{ }^{\circ} \mathrm{C}$ for the first two, and to $600{ }^{\circ} \mathrm{C}$ for the others. 


\subsubsection{Powder X-ray diffraction}

Variable temperature X-ray powder diffraction data were collected on $\mathbf{P N C}\left[\mathbf{c C}_{\mathbf{6 0}} \mathbf{C}^{-}\right.$ Co] and PNC[cbipy-Co] from $293 \mathrm{~K}$ to $500 \mathrm{~K}$ in approximately $25 \mathrm{~K}$ steps. The samples were loaded in glass capillaries, and mounted on a Bruker D8 diffractometer using $\mathrm{Cu} \mathrm{K} \alpha_{1}$ radiation $(\lambda=1.54056 \AA)$. Data were collected from 6 to $20^{\circ}$ in $2 \theta$. Pawley fits of these data were performed using TOPAS, in order to extract cell dimensions as a function of temperature.

\subsubsection{Chemical stability of $\mathrm{PNC}\left[\mathrm{CC}_{60}-\mathrm{Co}\right]$}

The chemical stability in water, acid and base of $\mathbf{P N C}\left[\mathbf{c C}_{\mathbf{6 0}}-\mathbf{C o}\right]$ crystals was examined. Some $\mathbf{P N C}\left[\mathbf{c C}_{\mathbf{6 0}}-\mathbf{C o}\right]$ crystals were suspended in $\mathrm{MeOH}$, which was then exchanged with water and the sample heated at $100{ }^{\circ} \mathrm{C}$ for $4 \mathrm{~h}$. The same sample was then split in three batches. The water was exchanged with $2 \mathrm{M} \mathrm{HCL}$ in one sample and with $2 \mathrm{M} \mathrm{NaOH}$ in another, then both were heated at $100{ }^{\circ} \mathrm{C}$ for $24 \mathrm{~h}$. The solid from each of the three samples, which looked macroscopically unchanged, was then washed and suspended in $\mathrm{MeOH}$. Crystals exposed to $\mathrm{H}_{2} \mathrm{O}$, $2 \mathrm{M} \mathrm{NaOH}$, and $2 \mathrm{M} \mathrm{HCl}$ were then isolated and indexed using single-crystal X-ray diffraction, collected on a Bruker ApexII diffractometer with graphitemonochromated Mo K $\alpha$ radiation $(\lambda=0.71073 \AA)$ at room temperature.

\subsubsection{Laboratory single crystal measurements}

For both PNC $\left[\mathbf{c C}_{60}-\mathbf{C o}\right]$ and $\mathbf{P N C}\left[\mathbf{c C}_{70}-\mathbf{C o}\right]$ single crystals were mounted onto a MiTiGen Microloops TM and a sphere of data collected on a Bruker SMART APEX II diffractometer with graphite-monochromated Mo K $\alpha$ radiation $(\lambda=0.71073 \AA)$ at $150 \mathrm{~K}$. These data were integrated using the program SAINT and the absorption correction was carried out using the program SADABS. ${ }^{46,47}$

\subsubsection{High-pressure crystallographic studies}

Compressibility studies were carried out on PNC[cbipy-Co], PNC $\left[\mathbf{c C}_{60}-\mathbf{C o}\right]$, $\mathbf{P N C}\left[\boldsymbol{c C}_{70}-\mathrm{Co}\right]$ and PNC[Co-vPy]. High-pressure single-crystal diffraction experiments were carried out on all four samples using methanol as a pressure transmitting media (PTM) until the samples became polycrystalline. The total pressure range for each experiment along with pressures to which structural 
refinements were possible are shown in Table 6.2. No structural refinements could be obtained for PNC[Co-vPy] so only unit cell parameters are reported.

A single crystal of each of the four compounds was loaded into a Merrill-Bassett diamond anvil cell with a half-opening angle of 40 degrees, composed of Boehler Almax diamonds with $600 \mu \mathrm{m}$ cutlet diamond anvils, a tungsten gasket and tungsten carbide backing plates. ${ }^{48} \mathrm{~A}$ small ruby chip was also loaded into the cell to act as a pressure calibrant, using the pressure dependent fluorescence of the ruby to measure the pressure. Diffraction data were collected on station I19 at the Diamond Light Source, Rutherford Appleton Laboratory, on a Rigaku Saturn 724 CCD detector using synchrotron radiation $(\lambda=0.5159 \AA$ ). Data collections were carried out using an exposure time and a step size of 1 second and 0.5 degrees respectively. The data were integrated using the programme SAINT using dynamic masks, these mask the regions of the detector which are shaded due to the pressure cell. ${ }^{46}$ Omission of shaded reflections, absorption correction and merging of data were carried out in a three-step process, firstly with the programme SHADE, then SADABS and finally XPREP. ${ }^{47,49,50}$

\subsubsection{High pressure structure refinements}

Structure refinements were carried out in CRYSTALS. ${ }^{51}$ For $\mathbf{P N C}\left[\mathbf{c C}_{60}-\mathbf{C o}\right]$, the (dipPhO) ${ }_{8} \mathrm{PcCo}_{c}$ group was refined anisotropically, while the encapsulated $\mathrm{C}_{60}$ was refined isotropically. All structures were refined against $F$ with an $\langle I / \sigma\rangle$ cut-off of 2. All 1,2 and 1,3 distances for the organic linker were restrained, whilst all torsion angles and metal - ligand bond distances were allowed to freely refine. The $C_{60}$ is disordered about the $\overline{4}$ axis. The best refinement results were obtained by assigning eight q-peaks within the cavity as C-atoms. 1,2 distances were then restrained to $1.54 \AA$ A. Vibrational and thermal similarity restraints were also applied to the organic linker and $\mathrm{C}_{60}$. Hydrogen atoms on the linker were placed geometrically and constrained to ride on their host atoms. The pore volume and electron count per unit cell (and therefore the solvent count) were calculated using the SQUEEZE algorithm in PLATON. ${ }^{52}$

For PNC $\left[\boldsymbol{c C}_{70}-\mathbf{C o}\right]$, the data were of particular low resolution (1.5 $\AA$ at best), and therefore no acceptable model for the $C_{70}$ could be obtained except for the first 
high-pressure data set, collected at $0.8 \mathrm{GPa}$. Above $0.8 \mathrm{GPa}$, we were still interested in the conformation of the $(\mathrm{dipPhO})_{8} \mathrm{PcM}$ group, so these data were refined against $F$ with $\langle I / \sigma\rangle$ cut-off of 2 , while both the included solvent and $C_{70}$ were modelled using the SQUEEZE algorithm in PLATON. All 1,2 and 1,3 distances for the organic linker were restrained whilst all torsion angles and metal - ligand bond distances were allowed to freely refine. Vibrational and thermal similarity restraints were also applied to the organic linker. Hydrogen atoms on the linker were placed geometrically and constrained to ride on their host atoms. The pore volumes were calculated using the SQUEEZE algorithm in PLATON. ${ }^{52}$

For PNC[cbipy-Co], the (dipPhO) $)_{8} \mathrm{PcM}$ group was refined anisotropically, along with bound bipy and pyridine molecules, which were disordered about the $\overline{4}$ axis. All structures were refined against $F$ with $\langle I / \sigma\rangle$ cut-off of 2 . All 1,2 and 1,3 distances for the organic linker were restrained, whilst all torsion angles and metal - ligand bond distances were allowed to freely refine. For PNC[Co-vPy] only unit cell dimensions are reported. 


\subsection{Results and Discussion}

The cubic structure of ( $\mathbf{d i p} \mathbf{P h O})_{8} \mathbf{P} \mathbf{c M}$ contains very large voids that account for $41 \%$ of the unit cell volume. ${ }^{*}$ However, until the success in stabilising the PNC with the inclusion of a suitably sized ligand, such as bipy, tying together two phthalocyanines across the cavity, the structure would collapse to an amorphous state upon desolvation. These molecular wall ties showed that the whole system can be stabilised by linking the phthalocyanine building units. The cavity that is bridged by the wall tie is almost spherical in shape due to the curved aromatic surfaces of the two phthalocyanine molecules. With a diameter of approximately $12 \AA$ this cavity was anticipated to be the perfect size and shape for the "ball and

(a)
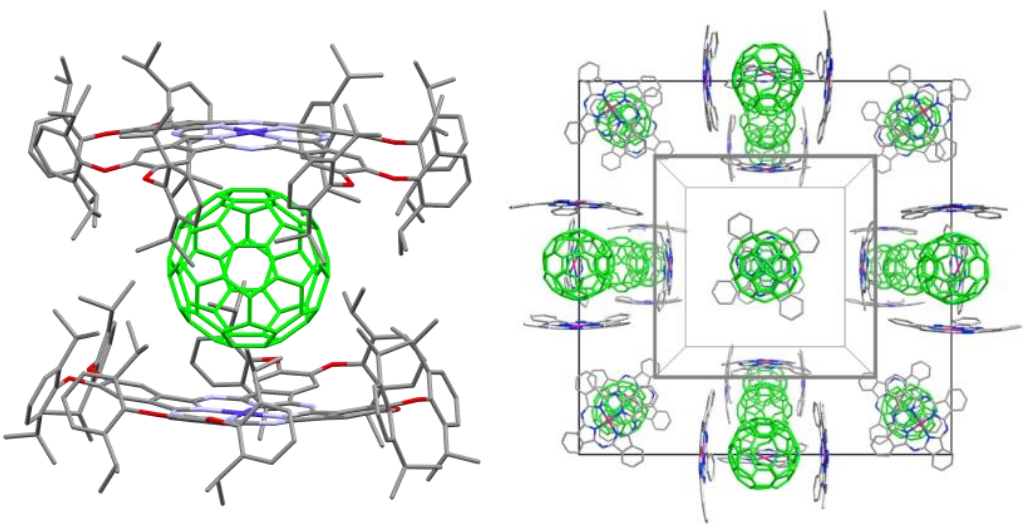

(b)
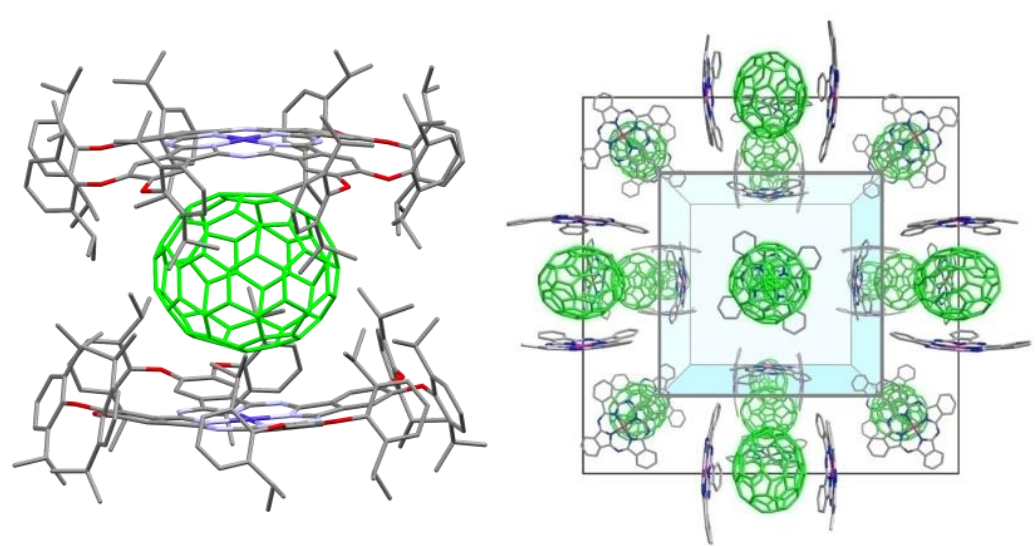

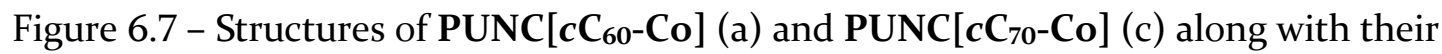
associated packing diagrams. Note the ellipsoid shape of the $C_{70}$ as opposed to the spherical $\mathrm{C}_{60}$. The hydrogens removed for clarity along with the phenoxy substituents for the packing diagrams.

${ }^{*}$ Void contact surface calculated using Mercury CSD 3.9. Probe radius $=1.2 \AA$, grid spacing $=0.7 \AA$, Contact surface $=21518.40 \AA^{3}$. 
socket" inclusion of a fullerene molecule that has previously been seen in other phthalocyanine systems. ${ }^{53-55}$

Co-crystallisation was successful through the diffusion of methanol into toluene solutions of cobalt derivative ( $\mathbf{d i p P h O})_{8} \mathrm{PcCo}$ and the fullerene $\mathrm{C}_{60}$ or $\mathrm{C}_{70}$, to yield crystals of $\mathbf{P N C}\left[\mathbf{c C}_{60}-\mathrm{Co}\right]$ and $\mathbf{P N C}\left[\mathbf{c C}_{70}-\mathrm{Co}\right]$. For both systems, large single crystals were grown and the presence of the fullerene was confirmed by laboratory single crystal X-ray diffraction (scXRD). Under ambient conditions $\mathbf{P N C}\left[\mathbf{c C}_{60}-\mathbf{C o}\right]$ and $\mathbf{P N C}\left[\mathrm{CC}_{70}-\mathrm{Co}\right]$ both crystallise in the cubic space group $P n \overline{3} n$ with a large unit cell ( $a=37.5761(2) \AA$ and $a=37.921(2) \AA$ respectively).

\subsubsection{Permanent Porosity}

The stability of fullerene-based PNCs to evacuation of the included solvent of crystallisation was the first step to confirming permanent porosity. To remove the solvent, crystals of $\mathbf{P N C}\left[\mathbf{c C}_{60}-\mathbf{C o}\right]$ were placed under vacuum or simply under a stream of nitrogen at room temperature. This was confirmed by thermal gravimetric analysis (TGA) of PUNC $\left[\mathbf{c C}_{60}-\mathbf{C o}\right]$ showing no mass loss at low temperatures that would be ascribed to solvent.

scXRD confirmed that both PUNC $\left[c_{60} \mathbf{C}_{60}-\mathbf{C o}\right]$ and PUNC $\left[\mathbf{c C}_{70}-\mathbf{C o}\right]$ retain the structure of their solvated counterparts and therefore both fullerenes stabilize the

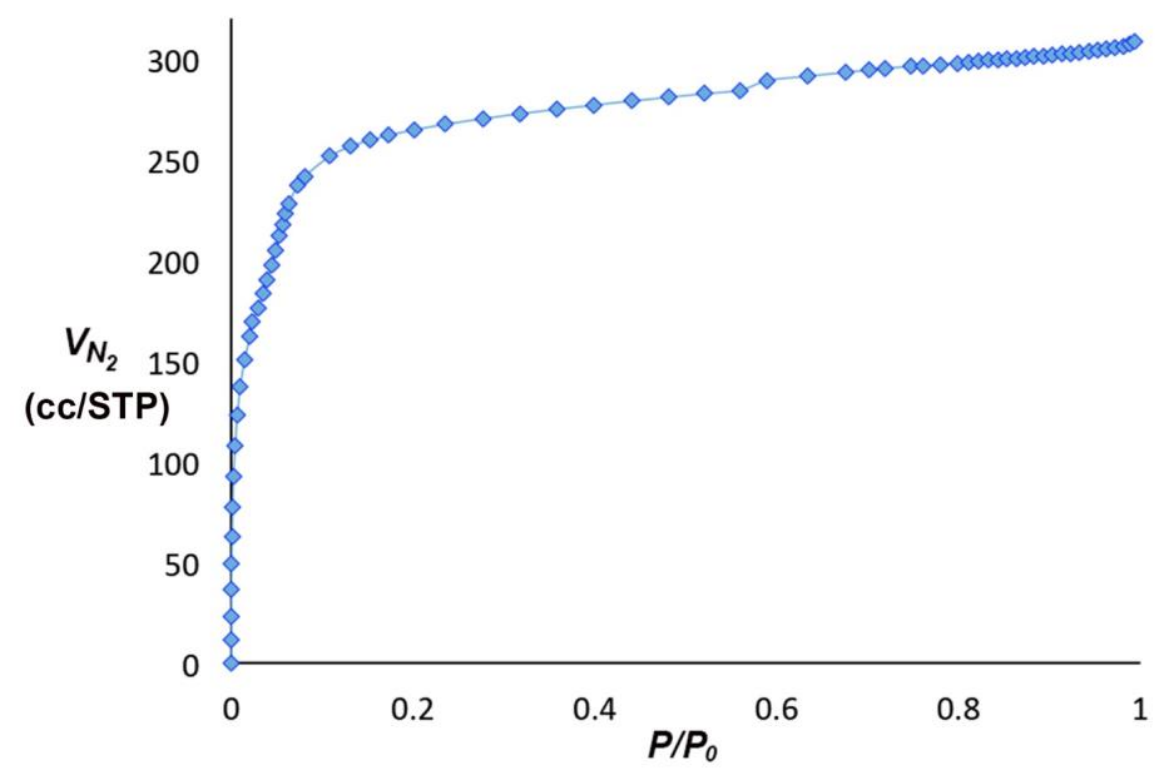

Figure 6.8 - Nitrogen adsorption isotherm for PUNC[cC60-Co]. 
PNC structure. Permanent porosity was confirmed by nitrogen adsorption at $77 \mathrm{~K}$ for PUNC $\left[\mathbf{c C}_{60}-\mathbf{C o}\right]$ and from this a BET surface area of $970 \mathrm{~m}^{2} \mathrm{~g}^{-1}$ was calculated, Figure 6.8 .

\subsubsection{Thermal stability}

The thermal stability of PUNC $\left[\mathbf{c C}_{60}-\mathbf{C o}\right]$ was assessed using TGA and compared to the wall tie stabilized PUNC[cbipy-Co], the unstablised (dipPhO) ${ }_{8} P \mathbf{P C o}$ and $\mathrm{C}_{60}$, Figure 6.9. Upon heating, a mass loss of $\sim 7 \%$ starting at $\sim 230{ }^{\circ} \mathrm{C}(503 \mathrm{~K})$ was observed for PUNC[cbipy-Co] that is due to the loss of the supporting bipyridyl ligand. For all three phathlocyanine samples a mass loss of $\sim 50 \%$ was observed starting at $\sim 430{ }^{\circ} \mathrm{C}(703 \mathrm{~K})$. This corresponds to the decomposition temperature of the phthalocyanine itself. PUNC $\left[\mathbf{c C}_{60}-\mathbf{C o}\right]$ under goes a further mass loss starting at $\sim 700{ }^{\circ} \mathrm{C}(973 \mathrm{~K})$ which is associated to the thermal decomposition of the fullerene.

Variable temperature powder X-ray diffraction ( $p \mathrm{XRD}$ ) showed that for $\mathbf{P N C}\left[\mathbf{c C}_{60} \mathbf{6}^{-}\right.$ Co] the crystal structure was unchanged upon heating to the maximum accessible temperature of 500 K, Figure 6.10. Pawley fits of these data show the unit cell dimensions smoothly increasing,.

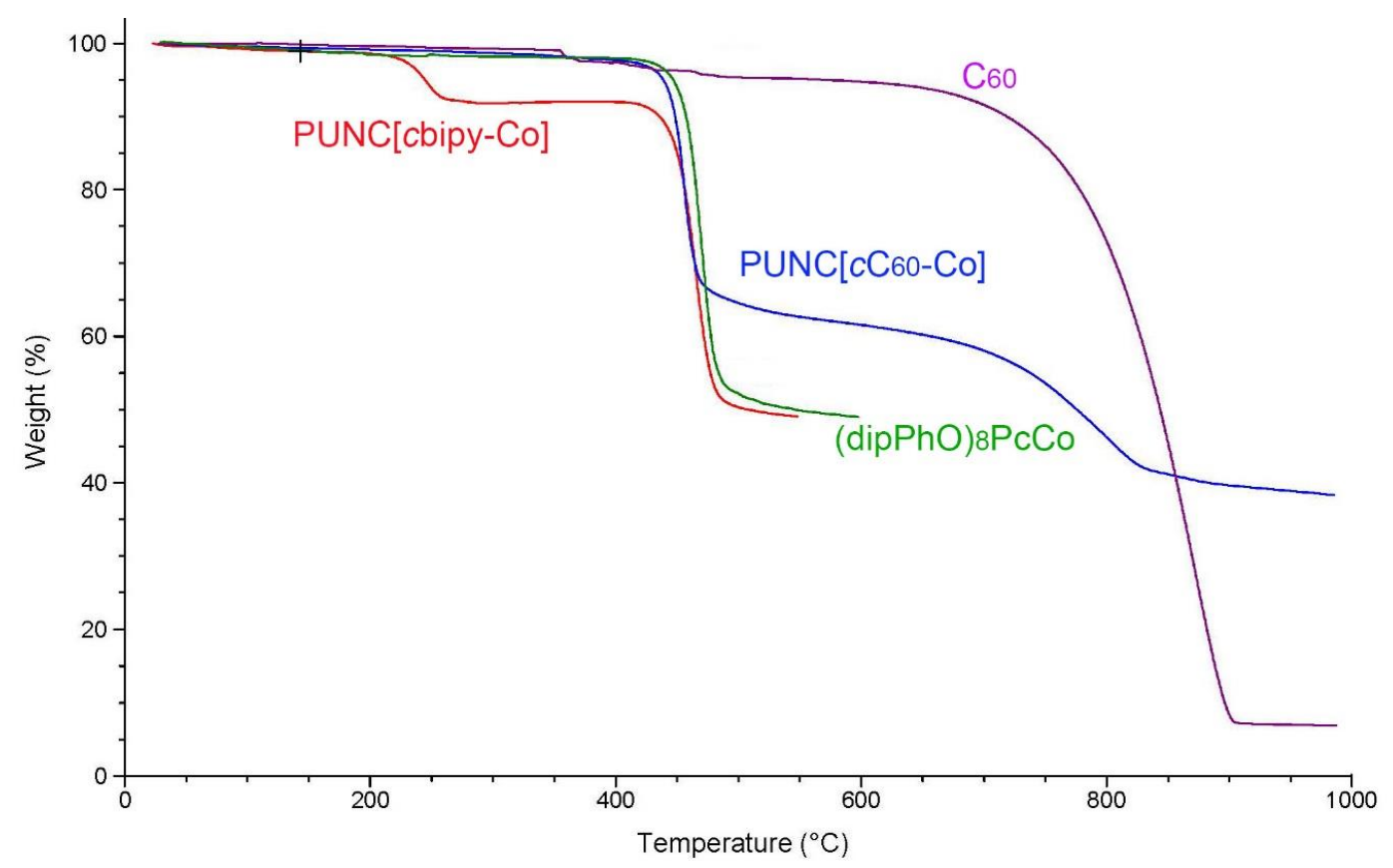

Figure 6.9 - TGA curves of $\mathrm{C}_{60}, \quad(\text { dipPhO })_{8} P c C o, \quad P U N C[c b i p y-C o]$, and PUNC $\left[\mathrm{cC}_{60}-\mathrm{Co}\right]$. 


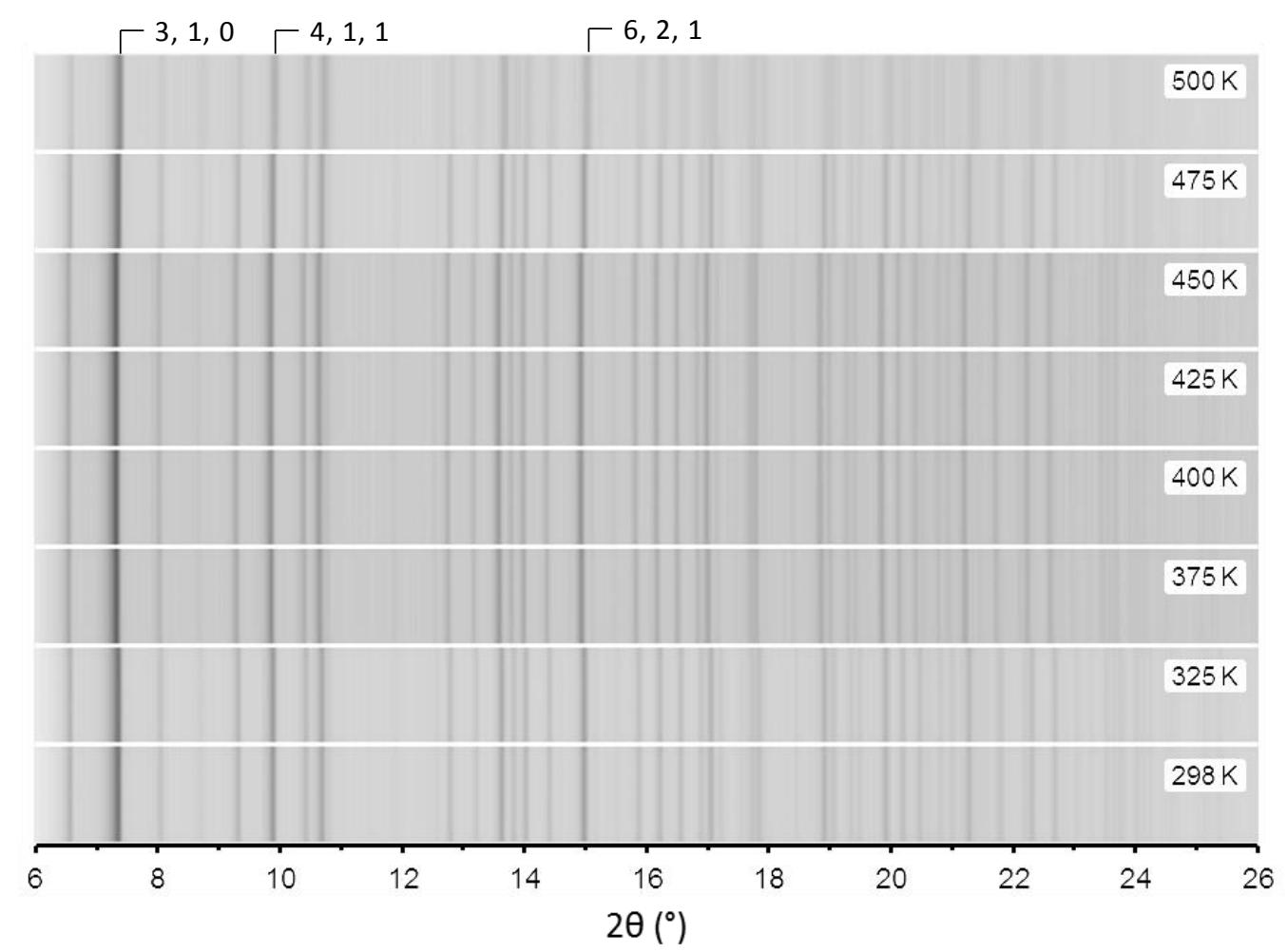

Figure $6.10-p X R D$ patterns displayed as a stacking plot for $\mathbf{P N C}\left[c^{-} \mathbf{C}_{60}-\mathbf{C o}\right]$ as a function of temperature.

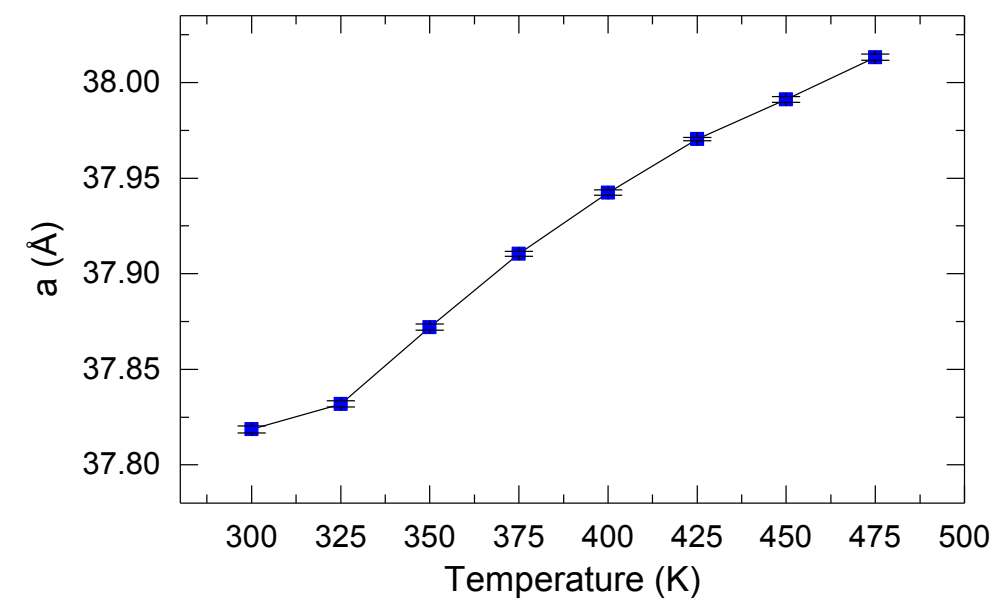

Figure 6.11 - Unit cell dimensions for $\mathbf{P N C}\left[c_{60} \mathbf{C}_{60}-\mathbf{C o}\right]$ as a function of temperature from Pawley refinements 
Variable temperature $p$ XRD for PUNC[cbipy-Co] showed that the loss of the bipy wall tie at 400 to $500 \mathrm{~K}$ shown in the TGA resulted in an irreversible transition above $475 \mathrm{~K}$ to a potentially new polymorph, Figure 6.12. The unit cell parameters of this new phase could not be determined from the powder pattern alone and attempts to index a recovered crystal were unsuccessful.

Expansion of the unit cell dimensions was observed between 300 and $500 \mathrm{~K}$, however, on plotting the fitted unit cell parameters on heating from 325 to $400 \mathrm{~K}$ the expansion plateaued, Figure 6.13. We propose that this inflection is indicative of losing one of the ligands from the Co-centre, probably caused by the removal of an axially bound species. The temperature that this is observed at in the crystallographic experiment does not coincide with any mass loss in the same temperature range in the TGA, however, these experiments we not performed on exactly the same samples.

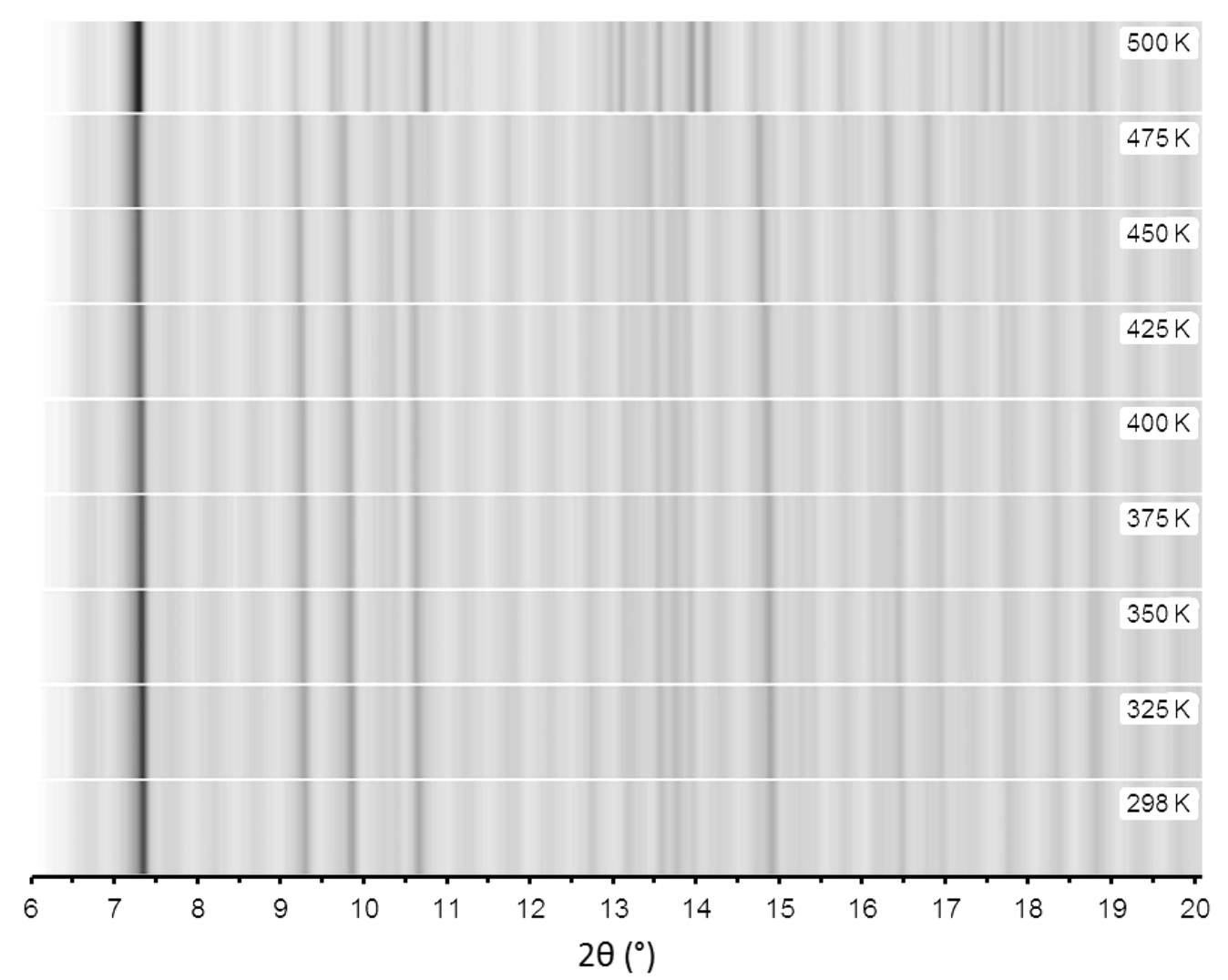

Figure 6.12 - pXRD patterns displayed as a stacking plot for PNC[cbipy-Co] as a function of temperature. Note the clear phase transitions on increasing temperature from 475 to $500 \mathrm{~K}$. 


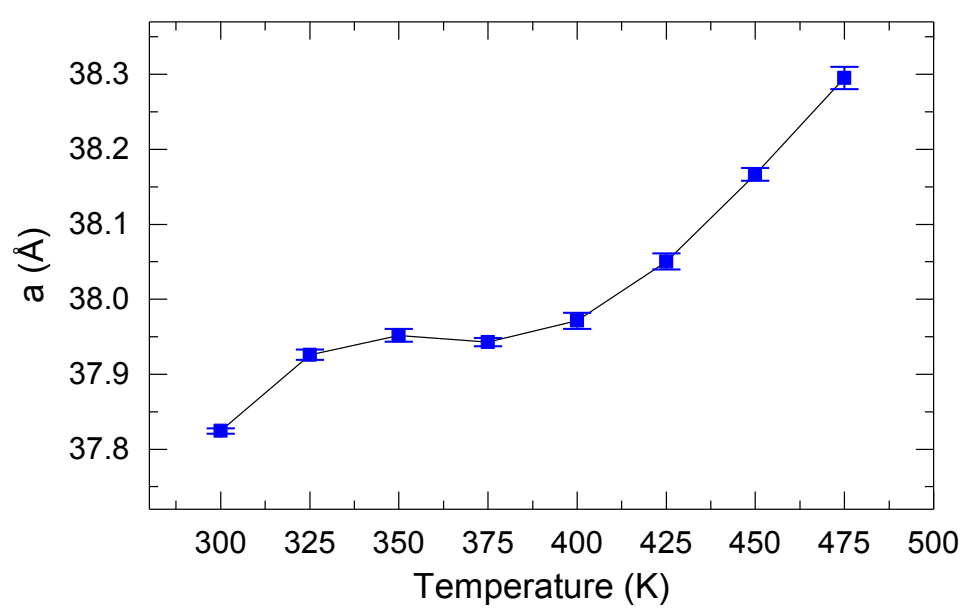

Figure 6.13 - Unit cell dimensions for PNC[cbipy-Co] as a function of temperature from Pawley refinements.

By using fullerene in place of a bipy wall tie the thermal stability improved significantly. $\mathbf{P N C}\left[\mathbf{c C}_{60}-\mathrm{Co}\right]$ was shown to remain crystalline to at least $500 \mathrm{~K}$. From the TGA, the sample appears stable up to the decomposition of the phthalocyanine itself (at around $700 \mathrm{~K}$ ). PNC[cbipy-Co] is compromised by the loss of the bipy ligand and irreversible transition to a new phase above $475 \mathrm{~K}$. The loss of the bipy wall tie at these elevated temperatures would inevitability have a destructive effect on the crystal. This was seen macroscopically, when trying to recover a single crystal from the powder, as the crystals had lost their distinct cubic morphology. 


\subsubsection{Chemical Stability}

For porous framework materials such as MOFs and COFs the greatest concern for the improvement of chemical stability has been the resistance, or complete lack of reactivity with water, either as a liquid or vapour. ${ }^{56}$ The metal-ligand bonds in MOFs for example are prone to hydrolysis under acidic or basic conditions.

There is no formal protocol for accessing chemical stability but it is important to understand how a material performs in hot acidic, and basic aqueous conditions. ${ }^{57}$ Crystals of $\mathrm{PNC}\left[\boldsymbol{c C}_{60}-\mathrm{Co}\right]$ demonstrated exceptional chemical stability by withstanding immersion in boiling water followed by boiling aqueous solutions of $2 \mathrm{M} \mathrm{NaOH}$ or $2 \mathrm{M} \mathrm{HCl}$ for $24 \mathrm{~h}$. Macroscopically the samples from each of the three experiments remained unchanged in appearance, with the crystallinity confirmed by scXRD, where each sample was indexed, Table 6.1.

Table 6.1 - Unit cell dimensions and volume of $\mathbf{P N C}\left[\mathbf{c C}_{60}-\mathbf{C o}\right]$ after soaking in water, $\mathrm{NaOH}$ and $\mathrm{HCl}$

\begin{tabular}{ccc}
\hline & $\begin{array}{c}\text { Unit cell } \\
\text { dimension }(\boldsymbol{A})\end{array}$ & $\begin{array}{c}\text { Unit cell } \\
\text { volume }\left(\boldsymbol{A}^{3}\right)\end{array}$ \\
\hline $\mathrm{H}_{2} \mathrm{O}$ & $37.46(1)$ & $52580(60)$ \\
$\mathrm{NaOH}$ & $37.60(1)$ & $53159(56)$ \\
$\mathrm{HCl}$ & $37.99(2)$ & $54848(87)$ \\
\hline
\end{tabular}

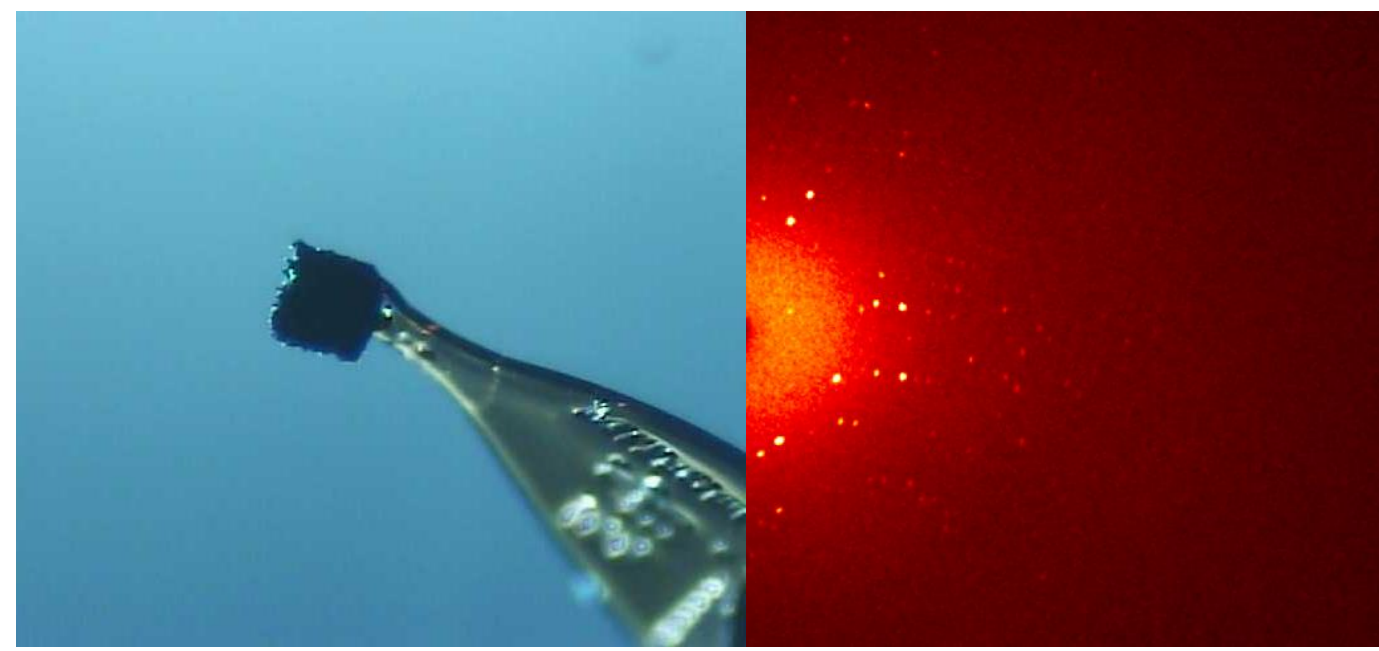

Figure 6.14 - Single-crystal micrograph and diffraction image of $\mathbf{P N C}\left[\mathbf{c C}_{60}-\mathbf{C o}\right]$ after soaking in boiling water. 


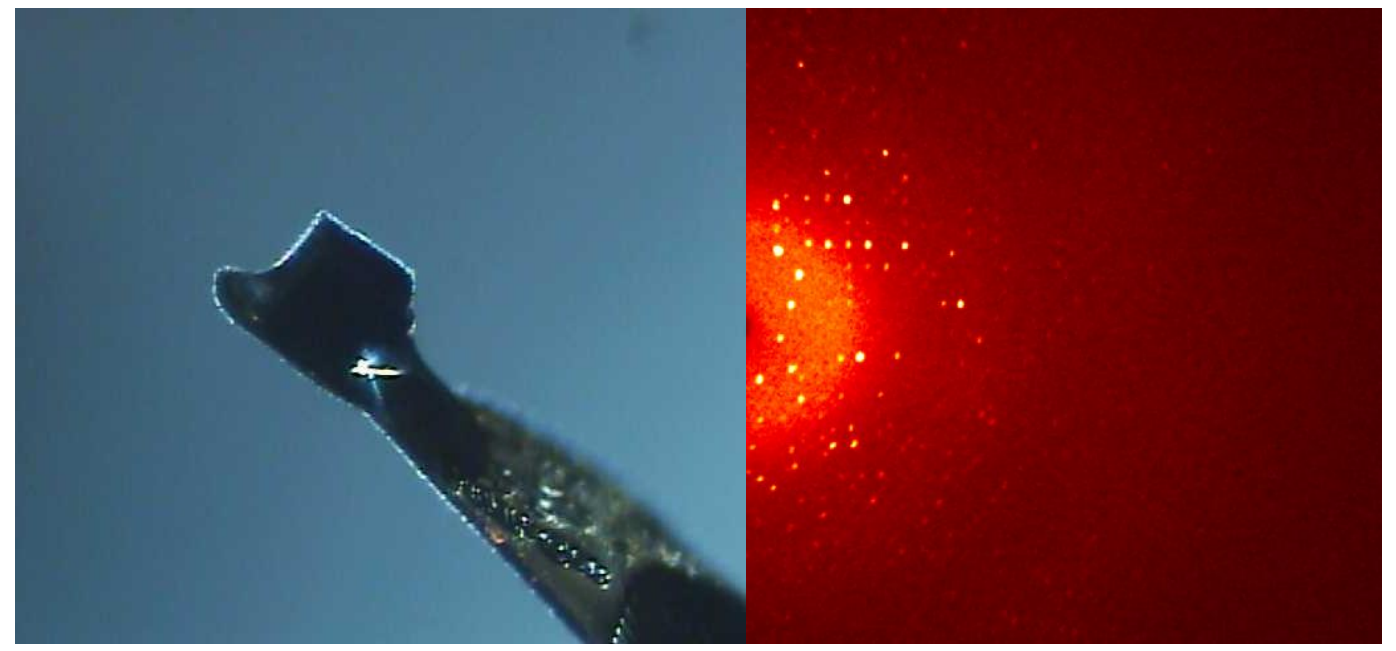

Figure 6.15 - Single-crystal micrograph and diffraction image of $\mathbf{P N C}\left[\mathbf{c C}_{60}-\mathbf{C o}\right]$ after soaking in $\mathrm{NaOH}$.

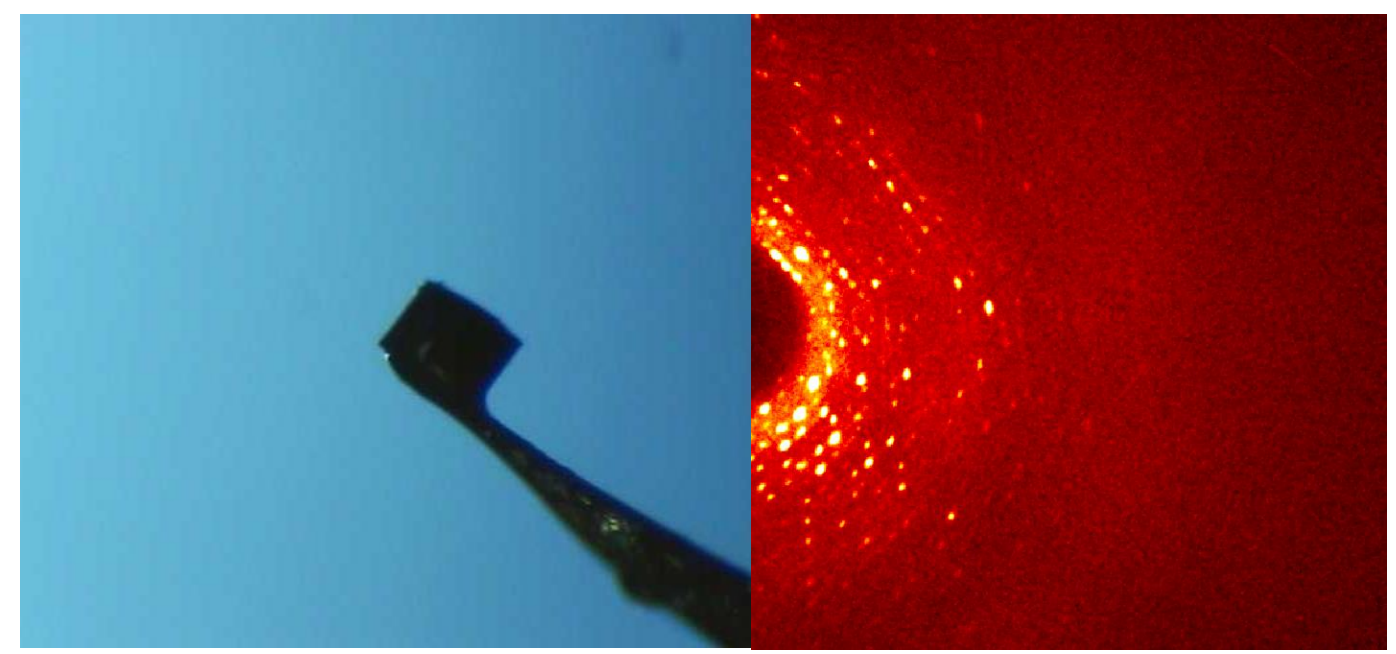

Figure 6.16 - Single-crystal micrograph and diffraction image of $\mathbf{P N C}\left[\boldsymbol{c C}_{60}-\mathbf{C o}\right]$ after soaking in $\mathrm{HCl}$. This sample is clearly not single however multi component crystals are not uncommon for $\mathbf{P N C}\left[\mathbf{c C}_{60}-\mathrm{Co}\right]$ but demonstrated the retention of crystallinity after soaking in $\mathrm{HCl}$. 


\subsubsection{High Pressure Experiments}

High pressure experiments on porous materials is a rapidly growing field. For MOFs, the pressure at which amorphisation occurs can also be used as an indicator of structural stability of the crystalline phase. ${ }^{58,59}$ There are a number of potential applications where stability in a high pressure environment would be key, such as gas separations or heterogeneous catalysis, and these studies can also reveal interesting anomalies in phase space, such as pressure induced phase transitions. ${ }^{60}$ However, to date there have been no high pressure structural stability studies conducted on a nanoporous molecular system.

Compressibility studies using high pressure scXRD were carried out on PNC [cbipy-Co], PNC $\left[c_{60} \mathrm{C}_{60}-\mathrm{Co}\right], \mathrm{PNC}\left[\mathrm{cC}_{70}-\mathrm{Co}\right]$ and PNC[Co-vPy], Table 6.2. This allowed a comparison between the unsupported structure and those stabilised by a molecular wall tie. In all cases methanol was used as a PTM and all four samples were compressed until they became amorphous, Figure 6.17.

Table 6.2 - Summary of high pressure scXRD experiments carried out using synchrotron radiation

\begin{tabular}{|c|c|c|}
\hline Compound & $\begin{array}{c}\text { Total pressure } \\
\text { range (GPa) }\end{array}$ & $\begin{array}{c}\text { Structural } \\
\text { refinements (GPa) }\end{array}$ \\
\hline $\mathrm{PNC}\left[c_{600}-\mathrm{Co}\right]$ & 0.10 to 4.53 & 0.10 to 3.88 \\
\hline $\mathrm{PNC}\left[c \mathrm{C}_{70}-\mathrm{Co}\right]$ & 0.08 to 5.85 & 0.08 to 2.67 \\
\hline PNC $[$ cbipy-Co $]$ & 0.14 to 3.06 & 0.14 to 0.96 \\
\hline PNC $[$ Co-vPy $]$ & 0.16 to 1.87 & - \\
\hline
\end{tabular}

Upon the application of pressure, diffraction from PNC[Co-vPy] immediately became too weak to obtain atomistic structural refinements, therefore only unit cell parameters could be reported to $1.87 \mathrm{GPa}$. On increasing pressure, the unit cell volume compressed by $6.74 \%$ to $1.87 \mathrm{GPa}$.

The bipy wall tie of PNC[cbipy-Co] afforded some stabilisation and atomistic * diffraction data were obtained up to $0.96 \mathrm{GPa}$. Above this pressure there was a

\footnotetext{
* The data are considered atomistic when collected to at least 1 Å resolution
} 


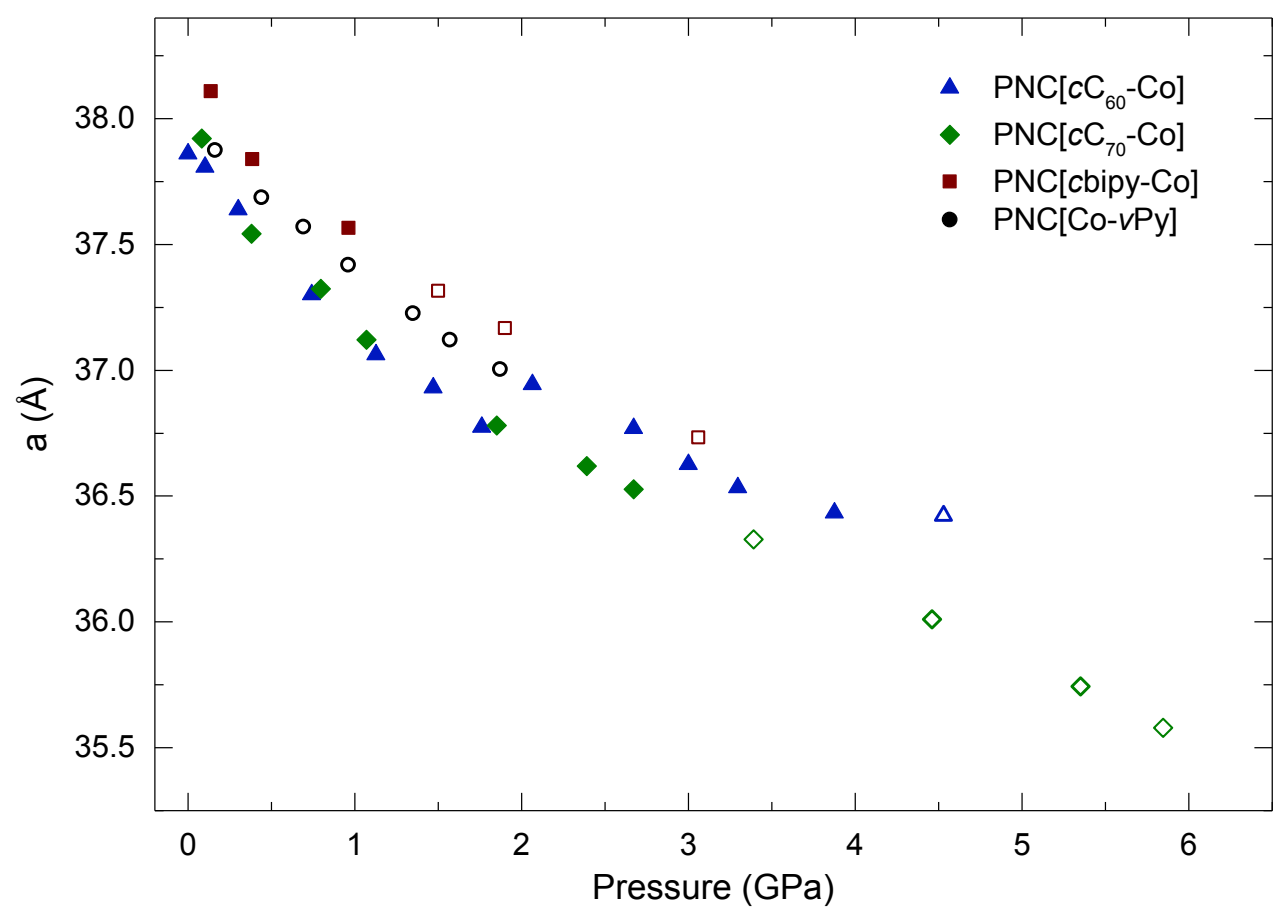

Figure 6.17 - Unit cell length as a function of increasing pressure for PNC[cbipy-Co],

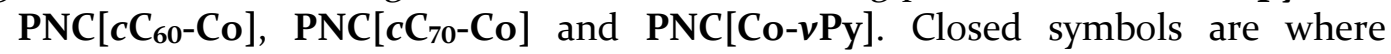
structural refinements were possible and open symbols show where only unit cell parameters could be extracted.

considerable reduction in the data quality, with the resolution dropping from 0.9 to $1.5 \AA$ An. Increasing the pressure above $3.06 \mathrm{GPa}$ resulted in total amorphisation of the sample. On increasing pressure to $3.06 \mathrm{GPa}$, the unit cell volume compressed by $10.45 \%$.

To understand the mechanism behind the pressure induced structural changes two key intermolecular distances were monitored, corresponding to the Co-to-Co distances across the nano-sized void and the fullerene/bipy containing cavity.

For PNC[cbipy-Co] the unit cell volume compressed by $4.21 \%(0.54 \AA)$ on increasing pressure from 0.14 to $0.96 \mathrm{GPa}$. Structurally this compression is almost entirely taken up by the compression of the Co-to-Co distance across the cavity, $95 \%$. The N-to-N distance of the bipy wall tie was only compressed by $0.12 \AA$ over this pressure range. However, the Co-N bonds connecting the bipy to the phthalocyanine were much more labile, reducing from 2.418 (13) $\mathrm{A}$ to 2.195 (10) $\AA$ between 0.08 and $0.95 \mathrm{GPa}$ respectively, causing the Co-Co distance to reduce by 
$0.515 \AA$ across the cavity. The remaining $5 \%$ reduction in unit cell is accounted for through the compression of the nano-sized void.

The fullerene containing $\mathbf{P N C}\left[\mathbf{c C}_{60}-\mathbf{C o}\right]$ and $\mathbf{P N C}\left[\boldsymbol{c C}_{70}-\mathbf{C o}\right]$ were both considerably more stable with diffraction data obtained up to 3.88 and $2.67 \mathrm{GPa}$ respectively. Amorphisation in both $\mathrm{PNC}\left[\mathbf{c C}_{60}-\mathrm{Co}\right]$ and $\mathrm{PNC}\left[\mathbf{c C}_{70}-\mathbf{C o}\right]$ occurred above 4.53 and $5.85 \mathrm{GPa}$ respectively. This puts these systems on a par with the more pressure-stable MOFs including the much denser ZIF-4, and the more porous HKUST-1, ${ }^{61,62}$, MOF-5, ${ }^{63} \mathrm{ZIF}-8^{64}$ and $\mathrm{Sc}_{2} \mathrm{BDC}_{3}{ }^{65}$.

PNC $\left[\mathbf{c C}_{70}-\mathbf{C o}\right]$ compressed smoothly on increasing pressure to $5.85 \mathrm{GPa}$, with the unit cell volume compressing by $10.97 \%$. Initially, $\mathbf{P N C}\left[\mathbf{c C}_{60}-\mathbf{C o}\right]$ followed a very similar compression curve to $1.76 \mathrm{GPa}$, however, on increasing pressure to 2.07

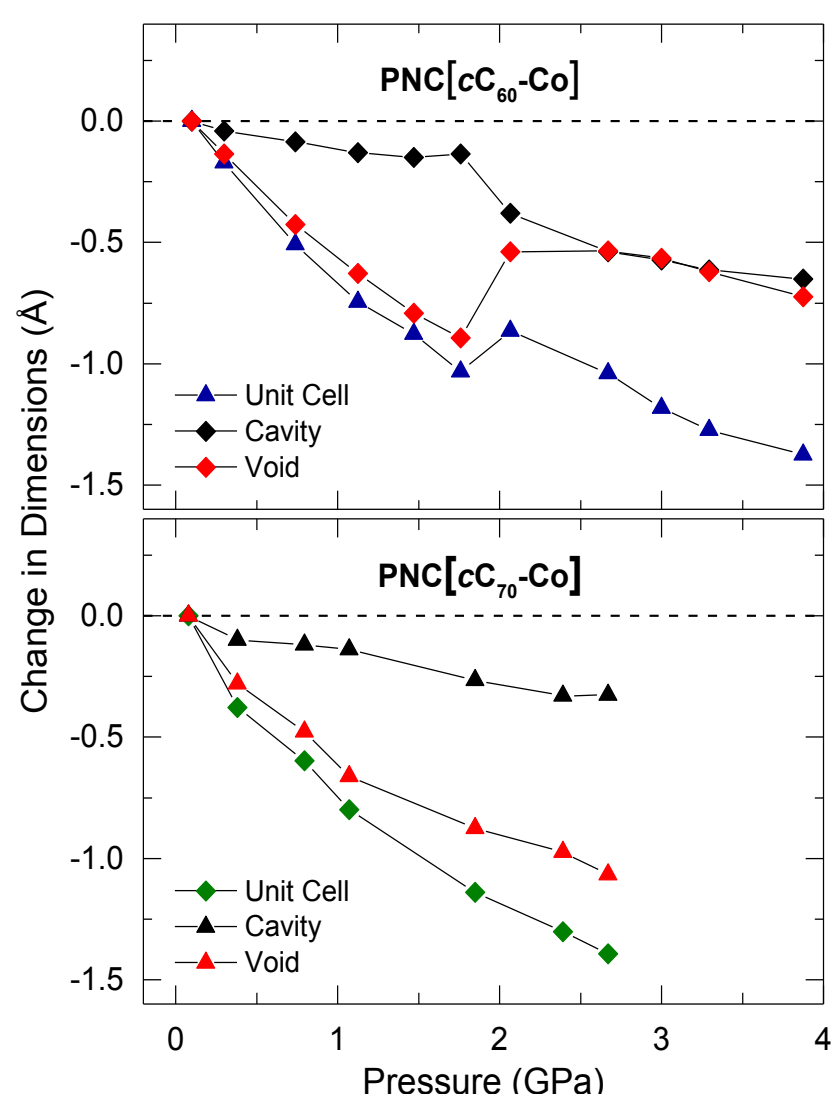

Figure 6.18 - Top graph: Changes in unit cell dimensions for PNC[cC60-Co], blue triangles, along with the Co-to-Co distance across the cavity and void, red and black diamonds respectively. Note the clear transition to $\mathrm{PNC}[\mathrm{cC60}-\mathrm{Co}]-\mathrm{II}$ above 1.76 GPa. Bottom graph: Changes in unit cell dimensions for $\mathrm{PNC}[\mathrm{cC} 70-\mathrm{Co}]$, green diamonds, along with the Co-to-Co distance across the cavity and void, red and black triangles respectively. 
GPa, a sharp increase in unit cell volume (1.39\%) occurred as the result of a phase transition to a previously unseen phase, hereafter referred to as $\mathbf{P N C}\left[\mathbf{c C}_{\mathbf{6 0}}-\mathbf{C o}\right]-\mathbf{I I}$, Figure 6.17.

For $\mathbf{P N C}\left[\mathbf{c C}_{70}-\mathbf{C o}\right]$ the smooth compression of the unit cell is accounted for by steady reductions in both the Co-to-Co distance across the void and cavity to 2.67 GPa, Figure 6.18. The compression across the void accounts for the majority of the compression $(77 \%)$ of the unit cell volume, unlike the cavity dominated compression found for PNC[cbipy-Co].

Up to $1.76 \mathrm{GPa}$, the unit cell volume of $\mathrm{PNC}\left[\mathrm{cC}_{60}-\mathrm{Co}\right]$ compressed smoothly by $7.98 \%$ (1.03 $\AA$ ) and, similar to $\mathbf{P N C}\left[\mathbf{c C}_{70}-\mathbf{C o}\right]$, this compression was dominated by the reduction in the Co-to-Co distance across the void (86\%). On increasing the pressure to $2.07 \mathrm{GPa}$ the transition to $\mathbf{P N C}\left[\mathbf{c C}_{60}-\mathbf{C o}\right]-\mathbf{I I}$ resulted in an increase in the void Co-to-Co distance of $0.36 \AA$, while the cavity Co-to-Co distance reduced by $0.24 \AA$.

On increasing the pressure further from 2.07 to $4.53 \mathrm{GPa}, \mathbf{P N C}\left[\mathbf{c C}_{60}-\mathbf{C o}\right]-\mathbf{I I}$ continued to be compressed smoothly, however, there was a distinct reduction in the compressibility of the Co-to-Co distance across the void, while compression across the cavity became more favourable, Figure 6.18. 
Intramolecular structural changes to the (dipPhO) ${ }_{8} P c C o$ building block of PNC $\left[\mathbf{c C}_{60}-\mathbf{C o}\right]$ occurred on increasing pressure and during the phase transition. Under ambient temperature and pressure, the phthalocyanine is not planner but slightly curved, with the centre being pushed into the void. We quantify this by measuring the out of plane distance, $\Delta$, between the phenoxy $\mathrm{O}$-atoms on the phthalocyanine and the metal centre (Figure 6.19).

Up to $1.76 \mathrm{GPa}, \Delta$ increased by $0.22 \AA$ giving a more curved phthalocyanine socket for the spherical $C_{60}$ to interact with. Throughout, the Co-to- $C_{60}$ distance and diameter of the $C_{60}$ between the two Co centres remained virtually unchanged at 3.03(2) $\AA$ and $\sim 6.58(5) \AA$. However, as $\Delta$ increased the di-iso-propylphenoxy groups that form the side walls of the cavity become very close to the $C_{60}$. The
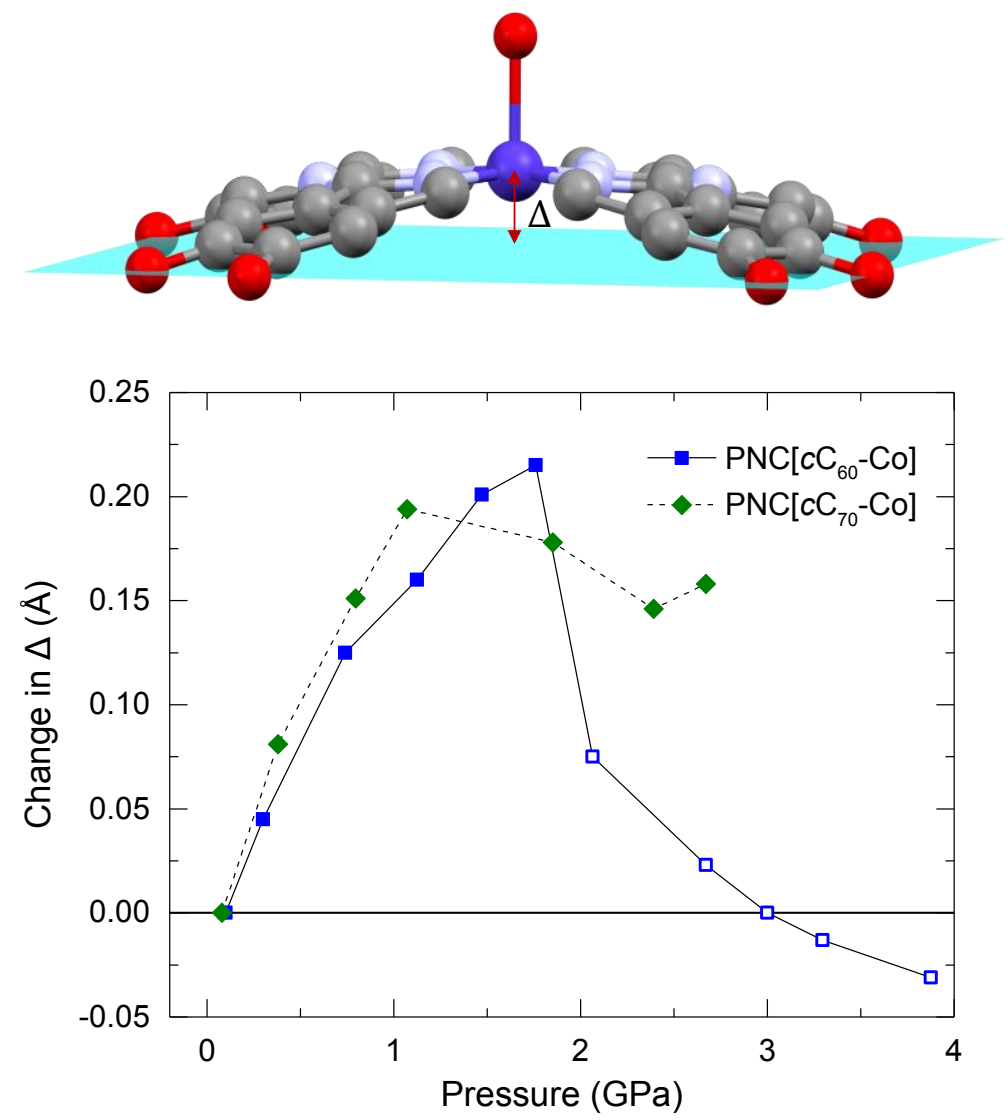

Figure 6.19 - The movement of the central Co atom out of the plane of the phthalocyanine, $\Delta$, with increasing pressure. The open symbols show the changes after the transition to PNC $\left[\mathbf{C C}_{60}\right.$-Co]-II. $\Delta$ - Defined as the distance between the Co atom and the mean plane of the eight phenoxy $\mathrm{O}$ atoms on the periphery of the phthalocyanine. The hydrogens, substituents, and a nitrogen atom where removed for clarity. 
shortest contact to the $\mathrm{C}_{60}$ measures $2.83 \AA$ at $0.10 \mathrm{GPa}$, and is reduced to $2.42 \AA$ by $1.76 \mathrm{GPa}$.

On undergoing the transition to $\mathbf{P N C}\left[\mathbf{c C}_{\mathbf{6 0}}-\mathbf{C o}\right]-\mathrm{II}$ at $2.07 \mathrm{GPa}, \Delta$ rapidly reduced shortening the Co-to-Co distance across the cavity by $0.24 \AA$, Figure 6.19. The reduction of $\Delta$ and the resultant flattening of the Pc caused the di-isopropylphenoxy groups to 'pull back' allowing the cavity diameter to expand by $\sim 0.7 \AA$. This was accompanied by a rotation of an iso-propyl group with the torsion angle of $\mathrm{C} 25-\mathrm{C} 26-\mathrm{C} 30-\mathrm{C} 32$ by $65^{\circ}$ (from $-47.1^{\circ}$ to $18.6^{\circ}$ ), Figure 6.20. The combination of the flattening Pc and the rotation of the iso-propyl group caused an increase the cavity diameter by $\sim 1.8 \AA$, Figure 6.21 .

While the $\mathrm{C}_{60}$ is restricted by the Co-to-Co distance across the cavity the increase of the cavity diameter changes the shape of the cavity and allows considerably more space for the $\mathrm{C}_{60}$ laterally. The effects of this are seen in the large increase in the average Ueq value $\left(0.26 \AA^{2}\right.$ to $\left.0.94 \AA^{2}\right)$ for the fullerene, which occurs after the phase transition, Figure 6.21. This is indicative of increased disorder or the fullerene starting to spin and move considerably more in the cavity. This is

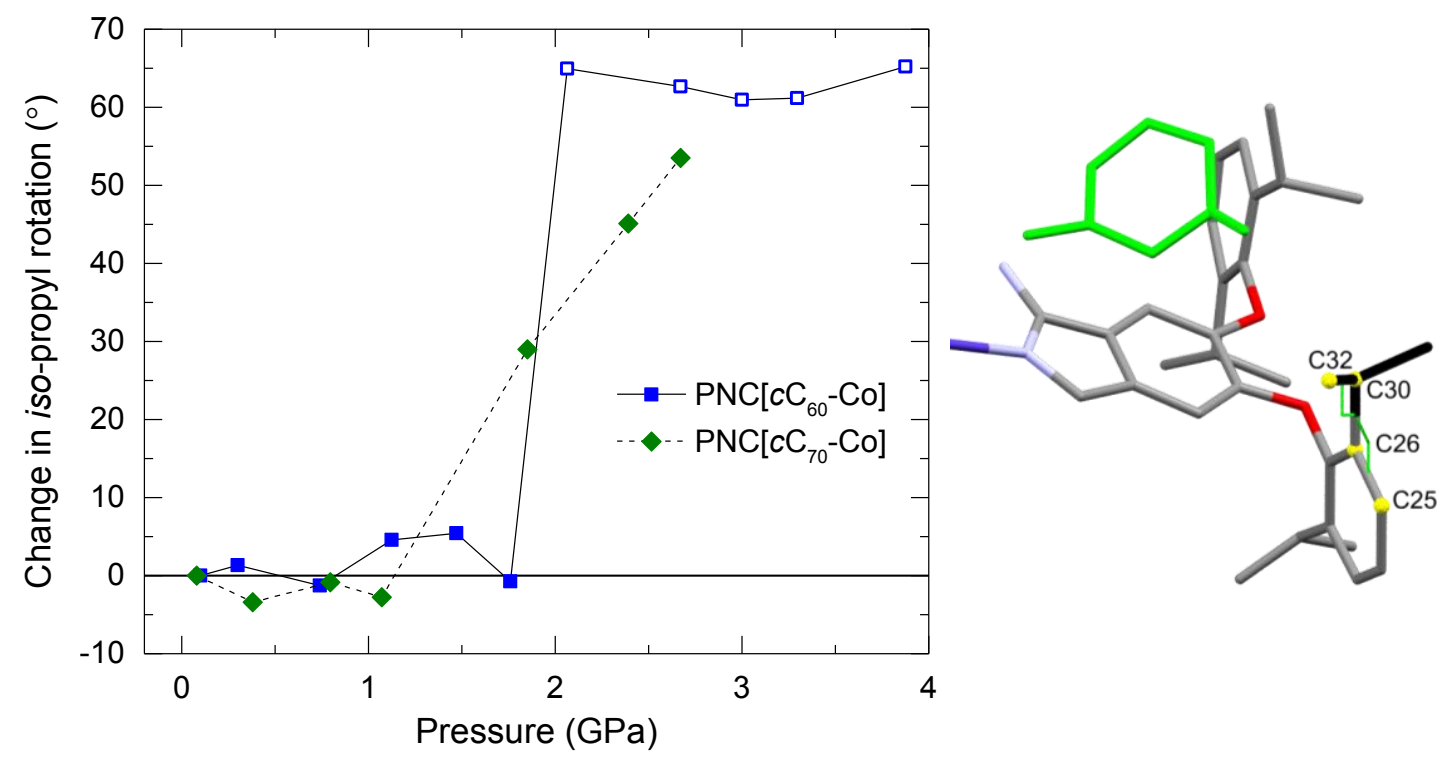

Figure 6.20 - The change in the C25-C26-C30-C32 torsion angle measuring the isopropyl rotation. The open symbols show the changes after the transition to PNC $\left[\mathbf{C C}_{60}\right.$-Co]-II. Insert of asymmetric unit with the torsion angle labelled on isopropyl group that rotates, shaded black. The $\mathrm{C}_{60}$ is shown in green and hydrogens have been removed for clarity. 
presumably caused by the shorter contacts between the Co metal centre of the phthalocyanine and fullerene, as the $\mathrm{C}_{60}$ is no longer being gripped so tightly by the close proximity of iso-propyl groups on the Pc. High-pressure low-temperature measurements could perhaps resolve the origin of this increased disorder; however, these measurements have not been performed here.

Further increasing the pressure on $\mathbf{P N C}\left[\mathbf{c C}_{60}-\mathbf{C o}\right]-\mathbf{I I}$ steadily reduced $\Delta$, the inverse of behaviour observed before the transition. The resultant decrease of the Co-to-Co distance across the cavity is almost entirely taken up by compression of

$$
\begin{aligned}
& \mathrm{PNC}\left[\mathrm{CC}_{60}-\mathrm{Co}\right] \\
& 1.76 \mathrm{GPa}
\end{aligned}
$$

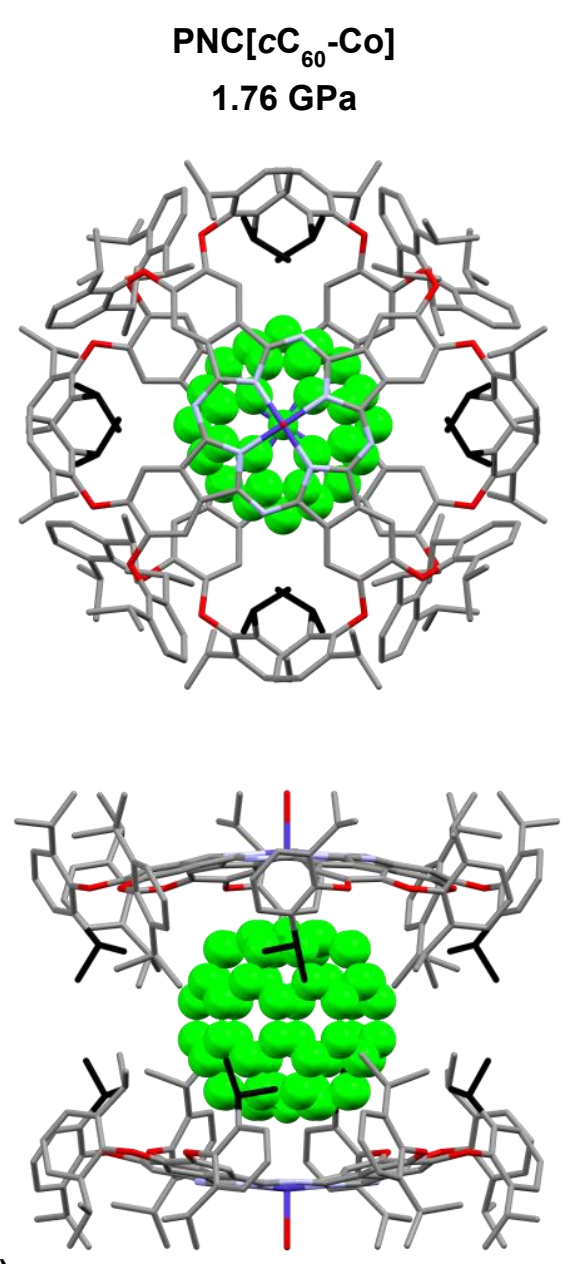

(a)

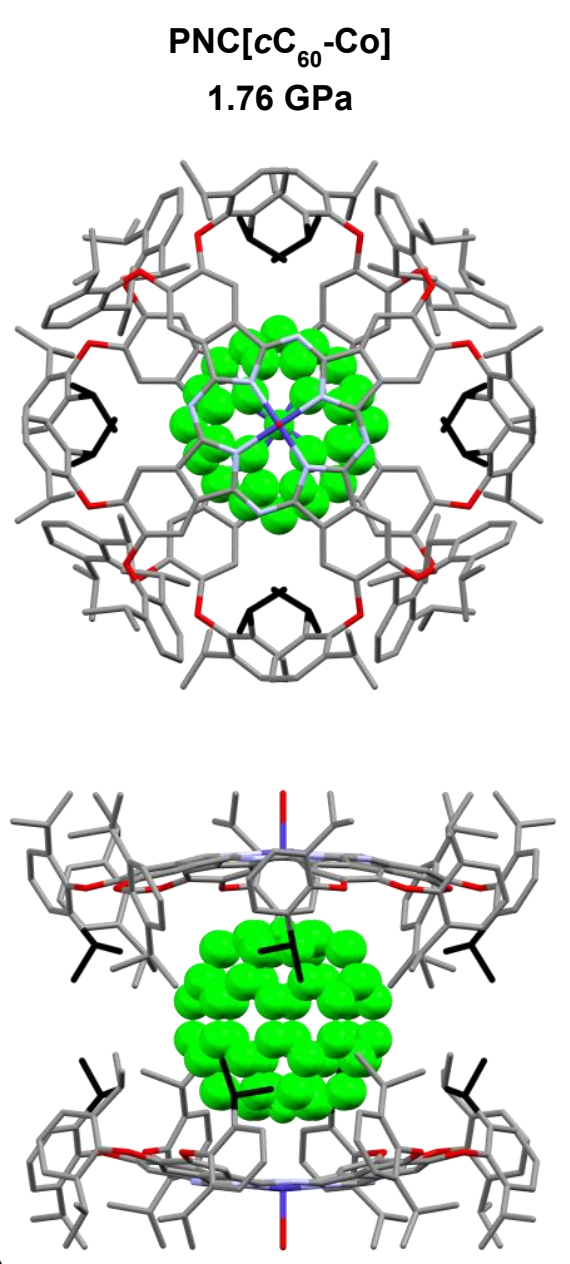

$\mathrm{PNC}\left[\mathrm{CC}_{60}-\mathrm{Co}\right]-\mathrm{II}$

$2.07 \mathrm{GPa}$

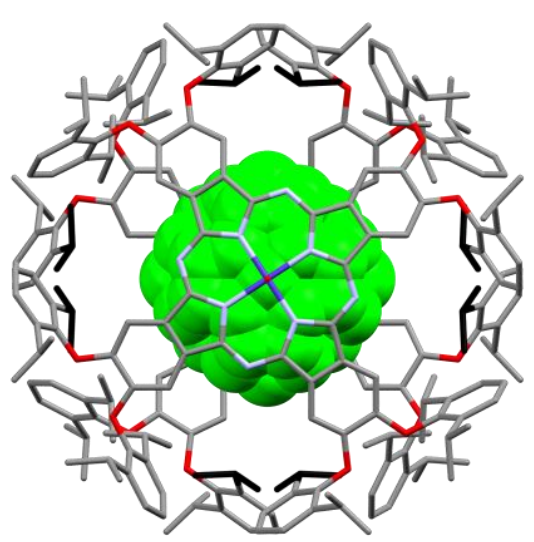

(b)

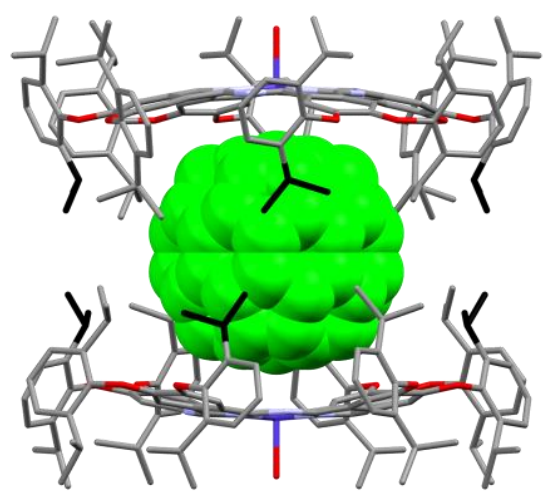

Figure 6.21 - Structural changes to the (dipPhO) $)_{8} \mathbf{P c C o}$ on undergoing the transition from $\mathbf{P N C}\left[\mathbf{C C}_{60}-\mathbf{C o}\right]$ to $\mathbf{P N C}\left[\mathbf{C C}_{60}-\mathbf{C o}\right]-\mathbf{I I}$ parallel to and perpendicular to the $\overline{4}$ axis. The iso-propyl group that undergoes conformation rearrangement is shaded black and the $\mathrm{C}_{60}$ is shown in green with ADPs set to $50 \%$ probability. Hydrogens have been removed for clarity. 
the $\mathrm{Co}-\mathrm{C}_{60}$ contact which shortens from $3.00 \AA$ to $2.86 \AA$ at 2.07 and $3.88 \mathrm{GPa}$ respectively.

By far the largest change during this transition was the increase in unit cell volume driven by re-inflation of the void, Figure 6.18. Previous high-pressure experiments on porous materials have shown similar behaviour with increases in volume on increasing pressure caused by swelling due to uptake of the PTM into the pores. This is a somewhat common phenomena in compression studies of MOFs, and has been observed in many different, well understood systems. ${ }^{63,64}$ In this case there appears to be a rapid uptake of the methanol PTM that causes the unit cell volume to expand, however, Due to the size of the void, $8 \mathrm{~nm}^{3}$, it proved impossible to resolve the contained disordered solvent with an atomistic model.

The SQUEZZE routine in PLATON was employed to model the residual electron density that in MOFs and other porous materials has be attributed to the solvent content in the pores. Unfortunately, in this case, the SQUEEZE output did not match the expected increase in residual electron density during the transition to PNC $\left[\mathbf{C C}_{60}-\mathbf{C o}\right]-$ II, Figure 6.22. SQUEEZE is sensitive to low resolution and missing data, both of which are common for high-pressure data sets. This is also by far the largest porous material ever studied at pressure attempt by SQUEEZE and so
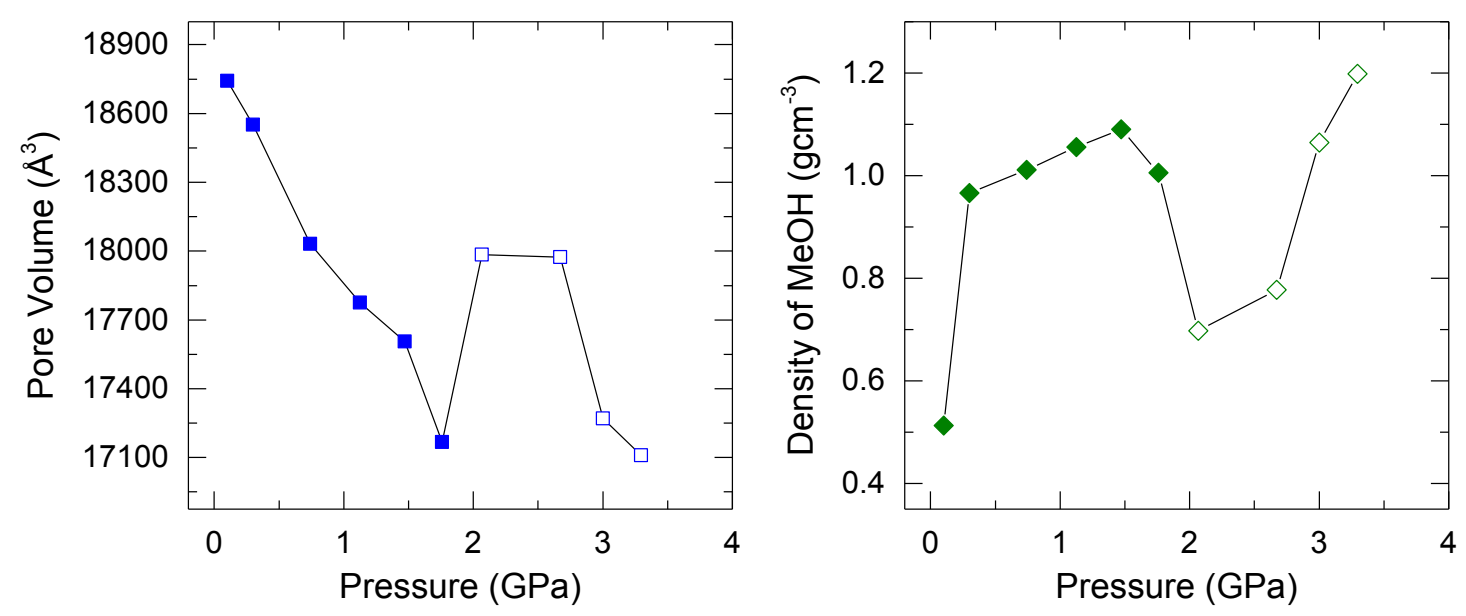

Figure 6.22 - Values calculated by SQUEEZE for total pore volume, left, and the density of $\mathrm{MeOH}$ in the pores of $\mathbf{P N C}\left[\mathbf{C C}_{60}-\mathbf{C o}\right]$ as calculated from the residual electron density. The open symbols show the changes after the transition to PNC $\left[\mathrm{CC}_{60}-\mathrm{Co}\right]-\mathrm{II}$. 
it is therefore unsurprising that disjointed trends in electron density in the pores as a function of pressure have been observed here.

In $\mathbf{P N C}\left[\mathbf{C C}_{60}-\mathbf{C o}\right]$ there are narrow channels that interconnect the cube shaped voids at each corner (calculated using a contact surface with probe radius of $1.2 \AA$ in Mercury). ${ }^{66}$ On increasing pressure to $1.67 \mathrm{GPa}$ these channels remain unchanged. On undergoing the transition to $\mathbf{P N C}\left[\mathbf{C C}_{60}-\mathbf{C o}\right]-\mathbf{I I}$ these channels become disconnected, as the di-iso-propylphenoxy groups rotate, removing the interconnections between the large nano-sized voids.

A similar phase transition was not observed on the compression of $\mathbf{P N C}\left[c_{70}-\mathbf{C o}\right]$ however there were still intramolecular distortions of the (dipPhO) ${ }_{8} \mathbf{P c C o}$. For PNC $\left[\mathbf{c C}_{70}-\mathbf{C o}\right] \Delta$ steadily increased by $0.19 \AA$ from 0.08 to $1.07 \mathrm{GPa}$, and upon further compression to $2.67 \mathrm{GPa}$, remained roughly unchanged, Figure 6.19. Above $1.07 \mathrm{GPa}$, a similar iso-propyl group rotation to that observed on undergoing the transition from $\mathbf{P N C}\left[\mathbf{C C}_{60}-\mathbf{C o}\right]-\mathbf{I}$ to $\mathbf{P N C}\left[\mathbf{C C}_{60}-\mathbf{C o}\right]-\mathbf{I I}$ was observed. In $\mathbf{P N C}\left[\mathbf{C C}_{\mathbf{7 0}}-\mathbf{C o}\right.$ ], the isopropyl group rotated by a comparable amount, $56^{\circ}$, but gradually from 1.07 to $2.39 \mathrm{GPa}$, Figure 6.20. Void analysis showed that the narrow channels interconnecting the voids reduced in diameter markedly, however the channels remained open throughout this pressure range, unlike in $\operatorname{PNC}\left[\mathrm{CC}_{60}-\mathrm{Co}\right]$.

The only difference between $\mathbf{P N C}\left[\mathbf{C C}_{60}-\mathbf{C o}\right]$ and $\mathbf{P N C}\left[\mathbf{C C}_{70}-\mathbf{C o}\right]$ is the size and shape of the fullerene. While $C_{60}$ is almost perfectly spherical $C_{70}$ has a more prolate shape akin to a rugby ball. This change in shape would make $C_{70}$ a closer match in size for the cavity leaving less room for lateral motion in the cavity preventing the large contraction in the Co-to-Co distance that was seen with the $\mathrm{C}_{60}$ included structure. Unfortunately due to the reduction of data quality associated with performing experiments at high pressure, the data for $\mathbf{P N C}\left[\mathbf{c C}_{70^{-}}\right.$ Co] were of low resolution (1.5 $\AA$ ). It therefore proved impossible to refine a stable model for the $C_{70}$, limiting further analysis of the interaction between the $C_{70}$ and the $(\operatorname{dipPhO})_{8} \mathrm{PcCo}$. 


\subsection{Conclusion}

The stability of porous molecular materials has been identified as one of the key factors required for the exploitation of their functionality and, ultimately, industrial utility. ${ }^{67}$ Although crystalline MOFs and COFs demonstrate reasonable thermal stability, only amorphous porous polymers are routinely stable towards strongly acidic or basic conditions. ${ }^{68}$ This is due to the need for reversible bond formation for the construction of crystalline nanoporous materials - bonds that are often labile in harsh hydrolytic environments, although zirconium-based MOFs demonstrate impressive stability. ${ }^{69,70}$ The efficient fabrication of cages, ${ }^{71}$ which are excellent components for making porous molecular crystals, also require the dynamic reversible formation of bonds and hence these too are prone to hydrolytic degradation unless stabilised by post-synthetic modification. ${ }^{41}$ Using only non-covalent forces to stabilise porous molecular materials may appear counter-intuitive but these interactions are not disrupted by hydrolytic conditions as seen with the exceptional stability of $\mathbf{P N C}\left[\mathbf{c C}_{60}-\mathbf{C o}\right]$ towards hydrolytic conditions. The crystal integrity was maintained fully, as determined by scXRD, following immersion of crystals in boiling water and boiling aqueous solutions of $2 \mathrm{M} \mathrm{NaOH}$ and $\mathrm{HCl}$ for $24 \mathrm{~h}$. The remarkable thermal and pressure stability of the fullerene-stabilised PNCs results from strong non-covalent interactions arising from intermolecular contacts over large surface areas. Similar intermolecular interactions are frequently found within co-crystals of fullerenes and substituted porphyrins, ${ }^{72,73}$ or phthalocyanine derivatives. ${ }^{74-76}$

The potential for fullerenes to be components for making a porous material was recognised soon after their discovery, ${ }^{77}$ however, $\mathbf{P N C}\left[\mathbf{c} \mathbf{C}_{60}-\mathbf{C o}\right]$ and $\mathbf{P N C}\left[\mathbf{c C}_{70}-\mathbf{C o}\right]$ are rare examples of their successful use for this purpose. ${ }^{78}$ Fullerenes were proposed as "stabilizing pillars" within porphyrin-based coordination frameworks, ${ }^{79-81}$ and a fullerene-based multidentate ligand has been used as a building block for a 2D coordination framework, ${ }^{82}$ but for these systems permanent porosity was not demonstrated.

In addition to providing exceptional stability, the structure of the PNCs provides intimate contact between of one of the most studied photoelectron 
donors (phthalocyanine) with the most studied class of photoelectron acceptor (fullerene). ${ }^{83,84}$ The combination of these highly functional components within a porous material suggests applications in light-harvesting, photovoltaics and photocatalysis, for which the stability demonstrated in this study will be beneficial. $^{85}$ 


\subsection{References}

1 Furukawa, H., Cordova, K. E., O'Keeffe, M. \& Yaghi, O. M. The Chemistry and Applications of Metal-Organic Frameworks. Science 341, 974, doi:10.1126/science.1230444 (2013).

2 Feng, X., Ding, X. S. \& Jiang, D. L. Covalent organic frameworks. Chemical Society Reviews 41, 6010-6022, doi:10.1039/c2cs35157a (2012).

3 McKeown, N. B. \& Budd, P. M. Exploitation of Intrinsic Microporosity in Polymer-Based Materials. Macromolecules 43, 5163-5176, doi:10.1021/mal006396 (2010).

4 Dawson, R., Cooper, A. I. \& Adams, D. J. Nanoporous organic polymer networks. Progress in Polymer Science 37, 530-563, doi:10.1016/j.progpolymsci.2011.09.002 (2012).

5 Lee, J., Farha, O. K., Roberts, J., Scheidt, K. A., Nguyen, S. T. \& Hupp, J. T. Metal-organic framework materials as catalysts. Chemical Society Reviews 38, 1450-1459, doi:10.1039/b807080f (2009).

6 Kreno, L. E., Leong, K., Farha, O. K., Allendorf, M., Van Duyne, R. P. \& Hupp, J. T. Metal-Organic Framework Materials as Chemical Sensors. Chemical Reviews 112, 1105-1125, doi:10.1021/cr200324t (2012).

7 Bildirir, H., Gregoriou, V. G., Avgeropoulos, A., Scherf, U. \& Chochos, C. L. Porous organic polymers as emerging new materials for organic photovoltaic applications: current status and future challenges. Materials Horizons 4, 546-556, doi:10.1039/C6MH00570E (2017).

8 Wu, M.-X. \& Yang, Y.-W. Applications of covalent organic frameworks (COFs): From gas storage and separation to drug delivery. Chinese Chemical Letters 28, 1135-1143, doi:10.1016/j.cclet.2017.03.026 (2017).

9 Zhang, W., Aguila, B. \& Ma, S. Potential applications of functional porous organic polymer materials. Journal of Materials Chemistry A 5, 8795-8824, doi:10.1039/C6TA11168H (2017).

10 Dunitz, J. D., Filippini, G. \& Gavezzotti, A. Molecular Shape and Crystal Packing: a Study of $\mathrm{C}_{12} \mathrm{H}_{12}$ Isomers, Real and Imaginary. Helvetica Chimica Acta 83, 2317-2335, doi:10.1002/1522-2675(20000906)83:9<2317::AIDHLCA2317>3.0.CO;2-R (2000). 
11 Dunitz, J. D. \& Gavezzotti, A. How molecules stick together in organic crystals: weak intermolecular interactions. Chemical Society Reviews 38, 2622-2633, doi:10.1039/B822963P (2009).

12 D., E. J., J., S. C. \& J., D. C. Synthesis and Applications of Porous Organic Cages. Chemistry Letters 44, 582-588, doi:10.1246/cl.150021 (2015).

13 Duriska, M. B., Neville, S. M., Lu, J., Iremonger, S. S., Boas, J. F., Kepert, C. J. \& Batten, S. R. Systematic Metal Variation and Solvent and HydrogenGas Storage in Supramolecular Nanoballs. Angewandte Chemie International Edition 48, 8919-8922, doi:10.1002/anie.200903863 (2009).

14 Ni, Z., Yassar, A., Antoun, T. \& Yaghi, O. M. Porous Metal-Organic Truncated Octahedron Constructed from Paddle-Wheel Squares and Terthiophene Links. Journal of the American Chemical Society 127, 12752 12753, doi:10.1021/ja052055c (2005).

15 Jones, J. T. A., Hasell, T., Wu, X. F., Bacsa, J., Jelfs, K. E., Schmidtmann, M., Chong, S. Y., Adams, D. J., Trewin, A., Schiffman, F., Cora, F., Slater, B., Steiner, A., Day, G. M. \& Cooper, A. I. Modular and predictable assembly of porous organic molecular crystals. Nature 474, 367-371, doi:10.1038/naturel0125 (2011).

16 McKeown, N. B. Molecular Nanoporous Crystals Predictable porosity. Nature Materials 10, 563-564, doi:10.1038/nmat3073 (2011).

17 Zhang, G., Presly, O., White, F., Oppel, I. M. \& Mastalerz, M. A Permanent Mesoporous Organic Cage with an Exceptionally High Surface Area. Angewandte Chemie International Edition 53, 1516-1520, doi:10.1002/anie.201308924 (2014).

18 Allcock, H. R. \& Siegel, L. A. Phosphonitrilic Compounds. III.1 Molecular Inclusion Compounds of Tris(o-phenylenedioxy)phosphonitrile Trimer. Journal of the American Chemical Society 86, 5140-5144, doi:10.1021/ja01077a019 (1964).

19 Cui, C., Shipman, P. R., Lalancette, R. A. \& Jäkle, F. Tris(2-pyridylborate) (Tpyb) Metal Complexes: Synthesis, Characterization, and Formation of Extrinsically Porous Materials with Large Cylindrical Channels. Inorganic Chemistry 52, 9440-9448, doi:10.1021/ic4010664 (2013). 
20 Lü, J., Perez-Krap, C., Suyetin, M., Alsmail, N. H., Yan, Y., Yang, S., Lewis, W., Bichoutskaia, E., Tang, C. C., Blake, A. J., Cao, R. \& Schröder, M. A Robust Binary Supramolecular Organic Framework (SOF) with $\mathrm{High}_{2} \mathrm{CO}_{2}$ Adsorption and Selectivity. Journal of the American Chemical Society 136, 12828-12831, doi:10.1021/ja506577g (2014).

21 Mastalerz, M. \& Oppel, I. M. Rational Construction of an Extrinsic Porous Molecular Crystal with an Extraordinary High Specific Surface Area. Angewandte Chemie International Edition 51, 5252-5255, doi:10.1002/anie.201201174 (2012).

22 Sozzani, P., Bracco, S., Comotti, A., Ferretti, L. \& Simonutti, R. Methane and Carbon Dioxide Storage in a Porous van der Waals Crystal. Angewandte Chemie International Edition 44, 1816-1820, doi:10.1002/anie.200461704 (2005).

23 Thomas, L. H., Cheung, E., Jones, A. O. F., Kallay, A. A., Lemée-Cailleau, M.-H., McIntyre, G. J. \& Wilson, C. C. 4-Phenoxyphenol: A Porous Molecular Material. Crystal Growth $\mathcal{E}$ Design 12, 1746-1751, doi:10.1021/cg200998u (2012).

24 Wang, S., Knowles, G. P., Chaffee, A. L. \& Langford, S. J. Selective $\mathrm{CO}_{2}$ uptake and vapor adsorption study within $\mathrm{Sn}(\mathrm{iv})$ porphyrin crystals. Crystengcomm 18, 1515-1522, doi:10.1039/C5CE02170G (2016).

25 Schneider, M. W., Oppel, I. M., Ott, H., Lechner, L. G., Hauswald, H.-J. S., Stoll, R. \& Mastalerz, M. Periphery-Substituted [4+6] Salicylbisimine Cage Compounds with Exceptionally High Surface Areas: Influence of the Molecular Structure on Nitrogen Sorption Properties. Chemistry - A European Journal 18, 836-847, doi:10.1002/chem.201102857 (2012).

26 Tozawa, T., Jones, J. T. A., Swamy, S. I., Jiang, S., Adams, D. J., Shakespeare, S., Clowes, R., Bradshaw, D., Hasell, T., Chong, S. Y., Tang, C., Thompson, S., Parker, J., Trewin, A., Bacsa, J., Slawin, A. M. Z., Steiner, A. \& Cooper, A. I. Porous organic cages. Nature Materials 8, 973-978, doi:10.1038/nmat2545 (2009).

27 McKeown, N. B. Nanoporous molecular crystals. Journal of Materials Chemistry 20, 10588-10597, doi:10.1039/C0JM01867H (2010). 
28 Endo, K., Sawaki, T., Koyanagi, M., Kobayashi, K., Masuda, H. \& Aoyama, Y. Guest-Binding Properties of Organic Crystals Having an Extensive Hydrogen-Bonded Network: An Orthogonal Anthracene-Bis(resorcinol) Derivative as a Functional Organic Analog of Zeolites. Journal of the American Chemical Society 117, 8341-8352, doi:10.1021/ja00137a007 (1995).

29 Nassimbeni, L. R. \& Kilkenny, M. L. Tetrakis(4aminopyridine)diisothiocyanatonickel(II) and its clathrates with EtOH, Me2CO and DMSO: structures, thermal stabilities and guest exchange. Journal of the Chemical Society, Dalton Transactions, 1172-1175, doi:10.1039/B010042K (2001).

30 Nassimbeni, L. R. Physicochemical Aspects of Host-Guest Compounds. Accounts of Chemical Research 36, 631-637, doi:10.1021/ar0201153 (2003).

31 Harris, K. D. M. Fundamental and Applied Aspects of Urea and Thiourea Inclusion Compounds. Supramolecular Chemistry 19, 47-53, doi:10.1080/10610270600977706 (2007).

32 Clements, M. \& le Roex, T. Structures and Inclusion Properties of Hydrogen-Bonded Host Frameworks Formed by 4,4'-(9Fluorenylidene)diphenol and Diamines. Crystal Growth E Design 16, 58695876, doi:10.1021/acs.cgd.6b00937 (2016).

33 Evans, J. D., Huang, D. M., Haranczyk, M., Thornton, A. W., Sumby, C. J. \& Doonan, C. J. Computational identification of organic porous molecular crystals. Crystengcomm 18, 4133-4141, doi:10.1039/C6CE00064A (2016).

34 Barbour, L. J. Crystal porosity and the burden of proof. Chemical Communications, 1163-1168, doi:10.1039/B515612M (2006).

35 Kaye, S. S., Dailly, A., Yaghi, O. M. \& Long, J. R. Impact of Preparation and Handling on the Hydrogen Storage Properties of $\mathrm{Zn}_{4} \mathrm{O}(1,4-$ benzenedicarboxylate) ${ }_{3}$ (MOF-5). Journal of the American Chemical Society 129, 14176-14177, doi:10.1021/ja076877g (2007).

36 Slater, A. G. \& Cooper, A. I. Function-led design of new porous materials. Science 348, 988, doi:10.1126/science.aaa8075 (2015).

37 Lim, S., Kim, H., Selvapalam, N., Kim, K.-J., Cho, S. J., Seo, G. \& Kim, K. Cucurbit[6]uril: Organic Molecular Porous Material with Permanent 
Porosity, Exceptional Stability, and Acetylene Sorption Properties. Angewandte Chemie International Edition 47, 3352-3355, doi:10.1002/anie.200800772 (2008).

38 Chen, T.-H., Popov, I., Kaveevivitchai, W., Chuang, Y.-C., Chen, Y.-S., Daugulis, O., Jacobson, A. J. \& Miljanic, O. S. Thermally robust and porous noncovalent organic framework with high affinity for fluorocarbons and CFCs. Nature Communications 5, doi:10.1038/ncomms6131 (2014).

39 Lee, N. H., Lee, D. W., Yeo, H., Kwak, K., Chun, H. S. \& Ok, K. M. A molecular porous zirconium-organic material exhibiting highly selective $\mathrm{CO}_{2}$ adsorption, high thermal stability, reversible hydration, facile ligand exchange and exclusive dimerization of phenylacetylene. Crystengcomm 16, 5619-5626, doi:10.1039/c4ce00253a (2014).

40 Nugent, P. S., Rhodus, V. L., Pham, T., Forrest, K., Wojtas, L., Space, B. \& Zaworotko, M. J. A Robust Molecular Porous Material with High $\mathrm{CO}_{2}$ Uptake and Selectivity. Journal of the American Chemical Society 135, 10950-10953, doi:10.1021/ja4054948 (2013).

41 Liu, M., Little, M. A., Jelfs, K. E., Jones, J. T. A., Schmidtmann, M., Chong, S. Y., Hasell, T. \& Cooper, A. I. Acid- and Base-Stable Porous Organic Cages: Shape Persistence and pH Stability via Post-synthetic "Tying" of a Flexible Amine Cage. Journal of the American Chemical Society 136, 7583-7586, doi:10.1021/ja503223j (2014).

42 McKeown, N. B., Makhseed, S., Msayib, K. J., Ooi, L.-L., Helliwell, M. \& Warren, J. E. A Phthalocyanine Clathrate of Cubic Symmetry Containing Interconnected Solvent-Filled Voids of Nanometer Dimensions. Angewandte Chemie International Edition 44, 7546-7549, doi:10.1002/anie.200502668 (2005).

43 Bezzu, C. G., Helliwell, M., Warren, J. E., Allan, D. R. \& McKeown, N. B. Heme-Like Coordination Chemistry Within Nanoporous Molecular Crystals. Science 327, 1627-1630, doi:10.1126/science.1184228 (2010).

44 Bezzu, C. G., Kariuki, B. M., Helliwell, M., Tuna, F., Warren, J. E., Allan, D. R. \& McKeown, N. B. In-situ coordination chemistry within cobalt- 
containing phthalocyanine nanoporous crystals. Crystengcomm 15, 15451550, doi:10.1039/C2CE26463C (2013).

45 Hupp, J. T. CRYSTAL ENGINEERING Towards artificial enzymes. Nat Chem 2, 432-433, doi:10.1038/nchem.678 (2010).

46 ECLIPSE (Madison, Wisconsin, USA, 2006).

47 SADABS Version 2008-1 (2008).

48 Moggach, S. A., Allan, D. R., Parsons, S. \& Warren, J. E. Incorporation of a new design of backing seat and anvil in a Merrill-Bassett diamond anvil cell. Journal of Applied Crystallography 41, 249-251, doi:10.1107/S0021889808000514 (2008).

49 SHADE (2004).

50 XPREP (Madison, Wisconsin, USA, 2004).

51 Betteridge, P. W., Carruthers, J. R., Cooper, R. I., Prout, K. \& Watkin, D. J. CRYSTALS version 12: software for guided crystal structure analysis. Journal of Applied Crystallography 36, 1487-1487, doi:10.1107/s0021889803021800 (2003).

52 Spek, A. L. Single-crystal structure validation with the program PLATON. Journal of Applied Crystallography 36, 7-13, doi:10.1107/s0021889802022112 (2003).

53 Bredenkötter, B., Henne, S. \& Volkmer, D. Nanosized Ball Joints Constructed from C60 and Tribenzotriquinacene Sockets: Synthesis, Component Self-Assembly and Structural Investigations. Chemistry - A European Journal 13, 9931-9938, doi:10.1002/chem.200700915 (2007).

54 Saegusa, Y., Ishizuka, T., Kojima, T., Mori, S., Kawano, M. \& Kojima, T. Supramolecular Interaction of Fullerenes with a Curved $\pi$-Surface of a Monomeric Quadruply Ring-Fused Porphyrin. Chemistry - A European Journal 21, 5302-5306, doi:10.1002/chem.201500389 (2015).

55 Sakaguchi, K.-i., Kamimura, T., Uno, H., Mori, S., Ozako, S., Nobukuni, H., Ishida, M. \& Tani, F. Phenothiazine-Bridged Cyclic Porphyrin Dimers as High-Affinity Hosts for Fullerenes and Linear Array of C60 in SelfAssembled Porphyrin Nanotube. The Journal of Organic Chemistry 79, 2980-2992, doi:10.1021/jo500034f (2014). 
56 Burtch, N. C., Jasuja, H. \& Walton, K. S. Water Stability and Adsorption in Metal-Organic Frameworks. Chemical Reviews 114, 10575-10612, doi:10.1021/cr5002589 (2014).

57 Howarth, A. J., Liu, Y., Li, P., Li, Z., Wang, T. C., Hupp, J. T. \& Farha, O. K. Chemical, thermal and mechanical stabilities of metal-organic frameworks. Nature Reviews Materials 1, 15018-15033, doi:10.1038/natrevmats.2015.18 (2016).

58 Coudert, F. X. Responsive Metal-Organic Frameworks and Framework Materials: Under Pressure, Taking the Heat, in the Spotlight, with Friends. Chemistry of Materials 27, 1905-1916, doi:10.1021/acs.chemmater.5b00046 (2015).

59 McKellar, S. C. \& Moggach, S. A. Structural studies of metal-organic frameworks under high pressure. Acta Crystallographica Section B 71, 587607, doi:10.1107/S2052520615018168 (2015).

60 McKellar, S. C., Sotelo, J., Greenaway, A., Mowat, J. P. S., Kvam, O., Morrison, C. A., Wright, P. A. \& Moggach, S. A. Pore Shape Modification of a Microporous Metal-Organic Framework Using High Pressure: Accessing a New Phase with Oversized Guest Molecules. Chemistry of Materials 28, 466-473, doi:10.1021/acs.chemmater.5b02891 (2016).

61 Bennett, T. D., Simoncic, P., Moggach, S. A., Gozzo, F., Macchi, P., Keen, D. A., Tan, J. C. \& Cheetham, A. K. Reversible pressure-induced amorphization of a zeolitic imidazolate framework (ZIF-4). Chemical Communications 47, 7983-7985, doi:10.1039/clccl1985k (2011).

62 Graham, A. J., Tan, J.-C., Allan, D. R. \& Moggach, S. A. The effect of pressure on Cu-btc: framework compression vs. guest inclusion. Chemical Communications 48, 1535-1537, doi:10.1039/C1CC16045A (2012).

63 Graham, A. J., Allan, D. R., Muszkiewicz, A., Morrison, C. A. \& Moggach, S. A. The Effect of High Pressure on MOF-5: Guest-Induced Modification of Pore Size and Content at High Pressure. Angewandte Chemie International Edition 50, 11138-11141, doi:10.1002/anie.201104285 (2011).

64 Moggach, S. A., Bennett, T. D. \& Cheetham, A. K. The Effect of Pressure on ZIF-8: Increasing Pore Size with Pressure and the Formation of a High- 
Pressure Phase at 1.47 GPa. Angewandte Chemie International Edition 48, 7087-7089, doi:10.1002/anie.200902643 (2009).

Graham, A. J., Banu, A.-M., Dueren, T., Greenaway, A., McKellar, S. C., Mowat, J. P. S., Ward, K., Wright, P. A. \& Moggach, S. A. Stabilization of Scandium Terephthalate MOFs against Reversible Amorphization and Structural Phase Transition by Guest Uptake at Extreme Pressure. Journal of the American Chemical Society 136, 8606-8613, doi:10.1021/ja411934f (2014).

66 Macrae, C. F., Bruno, I. J., Chisholm, J. A., Edgington, P. R., McCabe, P., Pidcock, E., Rodriguez-Monge, L., Taylor, R., van de Streek, J. \& Wood, P. A. Mercury CSD 2.0 - new features for the visualization and investigation of crystal structures. Journal of Applied Crystallography 41, 466-470, doi:10.1107/S0021889807067908 (2008).

67 Slater, A. G. \& Cooper, A. I. Science. Science 348, 6238 (2015).

68 Mottillo, C. \& Friscic, T. Carbon Dioxide Sensitivity of Zeolitic Imidazolate Frameworks. Angewandte Chemie-International Edition 53, 7471-7474, doi:10.1002/anie.201402082 (2014).

69 Cavka, J. H., Jakobsen, S., Olsbye, U., Guillou, N., Lamberti, C., Bordiga, S. \& Lillerud, K. P. A new zirconium inorganic building brick forming metal organic frameworks with exceptional stability. Journal of the American Chemical Society 130, 13850-13851, doi:10.1021/ja8057953 (2008).

70 Feng, D. W., Gu, Z. Y., Li, J. R., Jiang, H. L., Wei, Z. W. \& Zhou, H. C. Zirconium-Metalloporphyrin PCN-222: Mesoporous Metal-Organic Frameworks with Ultrahigh Stability as Biomimetic Catalysts. Angewandte Chemie, International Edition 51, 10307-10310, doi:10.1002/anie.201204475 (2012).

71 Huang, S.-L., Jin, G.-X., Luo, H.-K. \& Hor, T. S. A. Engineering Organic Macrocycles and Cages: Versatile Bonding Approaches. Chemistry-an Asian Journal 10, 24-42, doi:10.1002/asia.201402634 (2015).

72 Bhyrappa, P. \& Karunanithi, K. Porphyrin-Fullerene, C-60, Cocrystallates: Influence of C-60 on the Porphyrin Ring Conformation. Inorganic Chemistry 49, 8389-8400, doi:10.1021/ic101030h (2010). 
73 Wang, Y. B. \& Lin, Z. Y. Supramolecular interactions between fullerenes and porphyrins. Journal of the American Chemical Society 125, 6072-6073, doi:10.1021/ja028998g (2003).

74 Wang, H., Qian, K., Qi, D., Cao, W., Wang, K., Gao, S. \& Jiang, J. Cocrystallized fullerene and a mixed (phthalocyaninato)(porphyrinato) dysprosium double-decker SMM. Chemical Science 5, 3214-3220, doi:10.1039/c4sc00694a (2014).

75 Konarev, D. V., Khasanov, S. S. \& Lyubovskaya, R. N. Fullerene complexes with coordination assemblies of metalloporphyrins and metal phthalocyanines. Coordination Chemistry Reviews 262, 16-36, doi:10.1016/j.ccr.2013.10.021 (2014).

76 Shimizu, S., Miura, A. \& Kobayashi, N. Highly deformed phthalocyanine as a suitable scaffold for pristine fullerenes. Crystengcomm 15, 3759-3762, doi:10.1039/c2ce26618k (2013).

77 Okeeffe, M. C60 ZEOLITES. Nature 352, 674-674, doi:10.1038/352674a0 (1991).

78 Minar, N. K., Hou, K., Westermeier, C., Doblinger, M., Schuster, J., Hanusch, F. C., Nickel, B., Ozin, G. A. \& Bein, T. A Highly-Ordered 3D Covalent Fullerene Framework. Angewandte Chemie-International Edition 54, 7577-7581, doi:10.1002/anie.201411344 (2015).

79 Taylor, S. K., Jameson, G. B. \& Boyd, P. D. W. A new polymeric framework formed by the self assembly of 5,10,15,20-tetra(3-pyridyl)porphyrin, HgI2 and C-60. Supramolecular Chemistry 17, 543-546, doi:10.1080/10610270500317342 (2005).

80 Boyd, P. D. W. \& Reed, C. A. Fullerene-porphyrin constructs. Accounts of Chemical Research 38, 235-242, doi:10.1021/ar040168f (2005).

81 Sun, D., Tham, F. S., Reed, C. A. \& Boyd, P. D. W. Extending supramolecular fullerene-porphyrin chemistry to pillared metal-organic frameworks. Proceedings of the National Academy of Sciences of the United States of America 99, 5088-5092, doi:10.1073/pnas.072602399 (2002).

82 Peng, P., Li, F. F., Neti, V., Metta-Magana, A. J. \& Echegoyen, L. Design, Synthesis, and X-Ray Crystal Structure of a Fullerene-Linked Metal- 
Organic Framework. Angewandte Chemie, International Edition 53, 160-163, doi:10.1002/anie.201306761 (2014).

83 Ragoussi, M.-E. \& Torres, T. Modern Synthetic Tools Toward the Preparation of Sophisticated Phthalocyanine-Based Photoactive Systems. Chemistry-an Asian Journal 9, 2676-2707, doi:10.1002/asia.201402311 (2014).

84 Bottari, G., Trukhina, O., Ince, M. \& Torres, T. Towards artificial photosynthesis: Supramolecular, donor-acceptor, porphyrin-and phthalocyanine/carbon nanostructure ensembles. Coordination Chemistry Reviews 256, 2453-2477, doi:10.1016/j.ccr.2012.03.011 (2012).

85 Bottari, G., de la Torre, G., Guldi, D. M. \& Torres, T. Covalent and Noncovalent Phthalocyanine-Carbon Nanostructure Systems: Synthesis, Photoinduced Electron Transfer, and Application to Molecular Photovoltaics. Chemical Reviews 110, 6768-6816, doi:10.1021/cr900254z (2010). 
- Chapter 7 -

Gas Absorption into Fullerene

Stabilised Phthalocyanine Porous

Molecular Crystals 


\subsection{Introduction}

Gas adsorptions studies are fundamental to the development of porous materials not least because revisable gas adsorption is an essential property of a truly porous material. ${ }^{1}$ Across all porous materials the majority of experimental studies involve gravimetric or volumetric adsorption measurements that give key information about the amount of gas adsorbed at specific pressures and the overall maximum uptake achievable. However, these methods do not reveal the location or the mechanism for adsorption in the pores. The need for such data can be seen in several examples where changes in the framework structure, and therefore pore size, shape and potential selectivity takes place on uptake of gas molecules. The Zn-based framework ZIF-8 (Zn(MeIm 2, MeIm = 2-methylimidazole) undergoes a phase transition on uptake of $\mathrm{N}_{2}$, which causes the channels in the structure to increase in size, mediated through rotation of the $\mathrm{MeIm}_{2}$ ligands. ${ }^{2}$ In-situ diffraction methods have consequently been shown to be a powerful tool in understanding gas uptake in MOFs. However, a comprehensive review of crystallographic studies of gas sorption in MOFs conducted by Carrington et al. in 2014 revealed just how few studies have taken place relative to the size of the field. ${ }^{3}$ In total Carrington et al. report on 47 different MOFs where the coordinates of the absorbed gas had been crystallographically determined while the CSD MOF subset contains more than 75,600 entrees. ${ }^{4,5}$ There are even fewer examples of porous molecular crystals (PMCs) when compared to other crystalline porous network materials such as MOFs and COFs and therefore even fewer structural gas adsorption studies. ${ }^{6}$

Here, we describe the stability of a fullerene stabilised phthalocyanine based porous molecular material to high-vacuum, and describe changes in the structure of this material when exposed to $\mathrm{O}_{2}$ and $\mathrm{CO}$ whilst simultaneously conducting insitu X-ray diffraction experiments on EH2 on station I19 at the Diamond Light Source using an in-situ gas cell, the design of which has been previously discussed $(\S 2.2)$. 


\subsection{Experimental}

Note: Synthesis of Cobalt 2,3,9,10,16,17,23,24-octa(2',6'-di-iso-propylphenoxy)phthalocyanine (dipPhO) ${ }_{8} P c C o$ and crystallisation of $\mathbf{P N C}\left[\mathbf{c C}_{60}-\mathrm{Co}\right]$ were carried out by $\operatorname{Dr}$ C. Grazia Bezzu and Mr Luke Burt from the University of Edinburgh.

\subsubsection{Synthesis}

The synthetic procedure for the formation of Cobalt 2,3,9,10,16,17,23,24-octa(2',6'di-iso-propylphenoxy)-phthalocyanine, (dipPhO) ${ }_{8} P \mathbf{P c o}$, was outlined previously in Chapter 6, §6.2.2.

\subsubsection{Crystallizations}

The cocrystallisation procedure for of $\mathbf{P N C}\left[\mathbf{c C}_{\mathbf{6 0}}-\mathbf{C o}\right]$ was outlined previously in Chapter 6, §6.2.3.

\subsubsection{PNC $\left[c \mathrm{C}_{60}-\mathrm{Co}\right]$ gas absorption}

A series of gas absorption experiments were carried out on $\mathrm{PNC}\left[\mathrm{cC}_{60}-\mathrm{Co}\right]$ using the gas cell set-up at Diamond Light Source, beamline I19. The set-up that was used is described in detail in Chapter 2. A single crystal of $\mathbf{P N C}\left[\mathbf{c C}_{60}-\mathbf{C o}\right]$ was affixed to a Mitegen ${ }^{\circledR}$ mount and placed inside a $1 \mathrm{~mm}$ outer diameter quartz capillary static cell. The crystals were picked dry and mounted using the smallest amount cyanoacrylate adhesive in order to avoid sample movement on application of gas pressure.

Data were collected for $\mathbf{P N C}\left[\mathbf{c C}_{60}-\mathbf{C o}\right]$ from high-vacuum (for at least 20 minutes, vacuum $=10^{-6}$ mbar) to 6.4 bar in $\mathrm{CO}$ and 8.1 bar in $\mathrm{O}_{2}$. The sample was then evacuated again for at least 10 minutes to ascertain the reversibility of gas uptake in the pores. All data were collected at $180 \mathrm{~K}$ while pressure changes and evacuation procedures were conducted at room temperature.

Data collections were carried out using an exposure time and a step size of $0.1 \mathrm{~s}$ and $0.4^{\circ}$ respectively. For each data set, a full sphere of data were collected. Data processing was carried out using the program Xia2, ${ }^{7}$ while the adsorption correction was carried out using the program AIMLESS. 8,9 
Structure refinements were carried out in CRYSTALS. ${ }^{10}$ All structures were refined against $F$ with an $\langle I / \sigma\rangle$ cut-off of 2 . All 1,2 and 1,3 distances for the organic linker and $\mathrm{C}_{60}$ were restrained, whilst all torsion angles and metal to ligand bond distances were allowed to freely refine. Vibrational and thermal similarity restraints were also applied to the organic linker and $C_{60}$. Hydrogen atoms on the linker were placed geometrically and constrained to ride on their host atoms. The pore volume and electron count per unit cell (and therefore the adsorbed gas content) were calculated using the SQUEEZE algorithm in PLATON. ${ }^{11}$

\subsubsection{Cambridge structural database (CSD) searches}

Searches of the Cambridge Structural Database ${ }^{4}$ utilized the program ConQuest ${ }^{12}$ version 1.19 with database updates to May 2017.

Survey of cobalt carbonyl complexes were performed using 5 coordinate Co bound to terminal carbonyl where only structures with 3D coordinates determined were used and excluding structures determined from powder. This returned $2214 \mathrm{Co}-$ Co distances from 604 unique structures.

Survey of cobalt dioxygen complexes were performed using Co bound end on to $\mathrm{O}_{2}\left(\eta^{1}-\mathrm{O}_{2}\right)$. Only structures with $3 \mathrm{D}$ coordinates determined were used and excluding structures determined from powder. This returned $17 \mathrm{Co}-\mathrm{O}_{\mathrm{O}_{2}}$ distances from 17 unique structures. 


\subsection{Results and Discussion}

As previously discussed in Chapter $6, \mathbf{P N C}\left[\mathbf{c C}_{60}-\mathbf{C o}\right]$ crystallises in the high symmetry, cubic space group $P n \overline{3} n$ with a large unit cell $(a \sim 37.8 \AA)$. The unit cell contains assemblies of two phthalocyanine molecules surrounding the fullerene $\left(\mathrm{C}_{60}\right)$ that then pack to leave very large, $\left(8 \mathrm{~nm}^{3}\right)$ solvent filled cubic voids. These voids are interconnected at each corner through channels of $\sim 4 \AA$ in diameter and have been shown to be accessible to gasses, through volumetric $\mathrm{N}_{2}$ adsorption/desorption experiments.

The coordination environment of the Co centre of $\mathbf{P N C}\left[\mathbf{c C}_{60}-\mathbf{C o}\right]$ under ambient conditions is 5 -coordinate, with four equatorial $\mathrm{Co}-\mathrm{N}$ bonds to the phthalocyanine and a water bound axially in the void. This water molecule can be removed by simply drying the crystals under a stream of nitrogen at room temperature to create an open metal site on the Co.

\subsubsection{CO adsorption}

To assess the uptake of $\mathrm{CO}$ into the void of $\mathbf{P N C}\left[\mathbf{c C}_{60}-\mathbf{C o}\right]$ data were initially collected on a crystal loaded into the gas cell surrounded by an ambient atmosphere of air, at $180 \mathrm{~K}$. From the refined structure the Co site on the face of the void was free from any axially bound species as the sample had been dried prior to collection. It was not possible to model the contents of the void using an atomistic model, therefore the SQUEEZE algorithm was employed to calculate the residual electron density of disordered void contents, Table 7.1

Table 7.1 - Summary of gas cell scXRD experiments using CO gas.

\begin{tabular}{ccc}
\hline Co Pressure (bar) & Co-C $c$ (A) & $\begin{array}{c}\text { SQUEEZE } \\
\text { (e-/unit cell) }\end{array}$ \\
\hline Ambient in air & N/A & 1271 \\
$1 \times 10^{-6}$ & N/A & 1489 \\
1.0 & $2.037(9)$ & 1723 \\
2.0 & $2.010(10)$ & 2161 \\
3.0 & $2.023(11)$ & 2444 \\
6.4 & $2.013(11)$ & 2315 \\
$1 \times 10^{-6}$ & $\mathrm{~N} / \mathrm{A}$ & 1246 \\
\hline
\end{tabular}


To assess the uptake of CO, the crystal was placed under high vacuum $\left(1 \times 10^{69}\right.$ bar) at room temperature for 32 minutes to fully evacuated the voids of $\mathbf{P N C}\left[\mathbf{c C}_{60} \mathbf{C o}^{-}\right.$ Co] of solvent. This is also standard practice to ensure the gas cell and lines are fully evacuated. Data were collected on $\mathbf{P N C}\left[\mathbf{c C}_{60}-\mathbf{C o}\right]$ under vacuum at $180 \mathrm{~K}$. Interestingly there was a slight increase in pore contents as modelled using SQUEEZE upon evacuation, though the increase was marginal.

Dosing the crystal with CO gas was performed incrementally. The first dosing was 1 bar of CO (0.99 atm). At this pressure, CO could be modelled bound to the Cometal centre in the axial position (i.e. the same position as the water ligand binds), Figure 7.2. The CO was found to bind linearly to the open Co site (O-C-Co angle $\left.=180^{\circ}\right)$ to yield what we refer to as $\mathbf{P N C}\left[\mathbf{c C}_{60}-\mathbf{C o}-v \mathbf{C O}\right]$. The $\mathrm{CO}$ was determined to be fully occupied with a Co- $\mathrm{C}_{\mathrm{CO}}$ distance measuring 2.037(9) $\AA$. From a survey of the Cambridge Structural Database (CSD version 5.38, May 2017), single-crystal structures of cobalt carbonyl complexes with $\mathrm{Co}_{\mathrm{C}} \mathrm{C}_{\mathrm{co}}$ distances longer than $2.0 \AA$ are very unusual. $\mathrm{Co}(\mathrm{II})$ carbonyl complexes almost all exhibit strong $\mathrm{Co}-\mathrm{CO}$ bonds with a Co- $\mathrm{C}_{\text {co }}$ distances around 1.8 Å, Figure 7.1.

\section{Co-CO Distance}

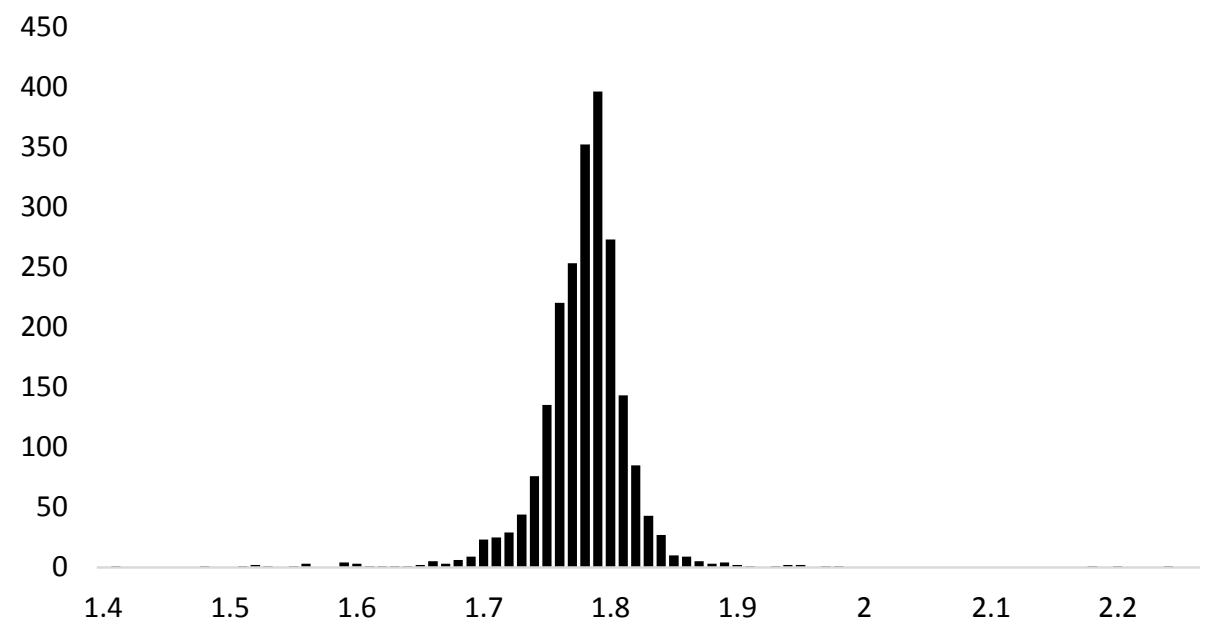

Figure 7.1 - Histogram of the Co-CO distance of 5 coordinate Co (CSD, May 2017). Based on 2214 distances from 604 unique structures. 


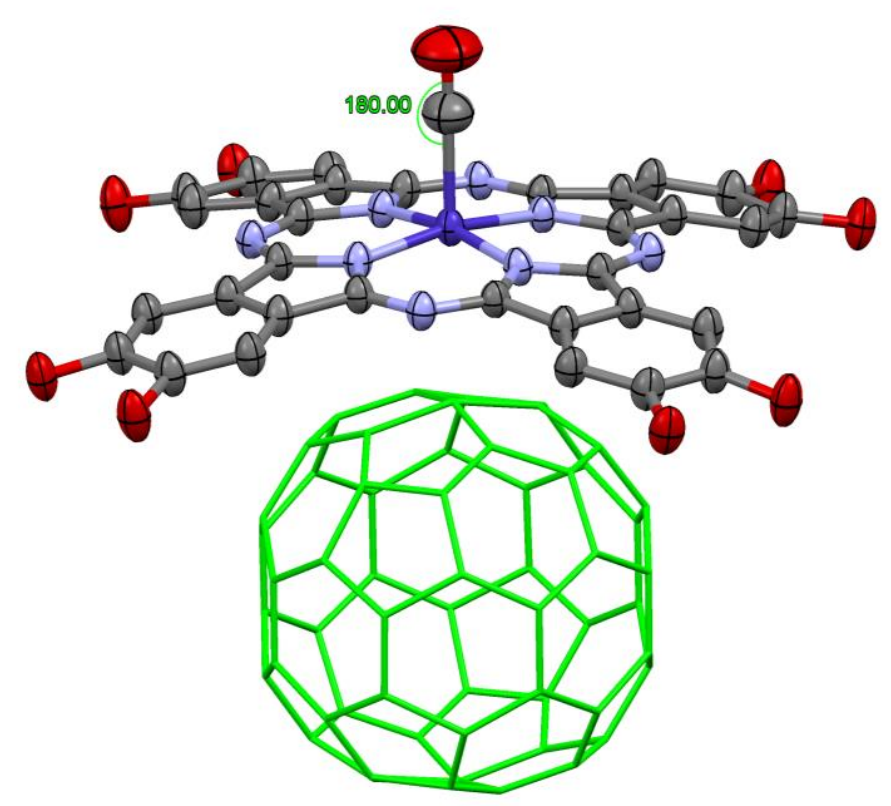

Figure 7.2 - Structure of $\mathbf{P N C}\left[\boldsymbol{c C}_{60}-\mathbf{C o}-\boldsymbol{v} \mathbf{C O}\right]$ at a $\mathrm{CO}$ gas pressure of 1 bar. The substituents groups on the phthalocyanine and hydrogens have been hidden for clarity. The C60 is shown green.

In a recent study, Gonzalez et al. investigated the gas adsorption for the cobalt MOF, $\mathrm{Co}_{2}$ (dobdc), using similar in situ techniques to those presented here. ${ }^{13}$ $\mathrm{Co}_{2}$ (dobdc) has open metal sites on the coordinative unsaturated Co metal centres that face into the channels and are analogous to the open Co sites found in PNC $\left[\boldsymbol{c C}_{60}-\mathbf{C o}\right]$. Gonzalez et al. found that $\mathrm{CO}$ bound to the open Co site of

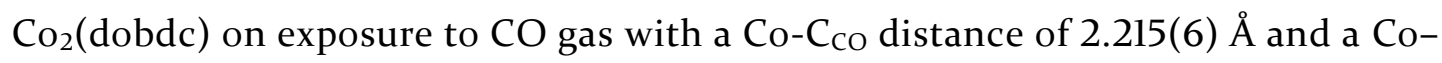
$\mathrm{C}-\mathrm{O}$ binding angle ranging from $176^{\circ}$ to $178^{\circ}$. The long $\mathrm{Co}_{\mathrm{O}} \mathrm{C}_{\mathrm{CO}}$ distance, and large displacement parameters for the oxygen atom of the bound $\mathrm{CO}$, are both indicative of a weak Co(II)-CO interaction with little $\pi$ backbonding that frees the bound $\mathrm{CO}$ to deflect along the $\mathrm{Co}(\mathrm{II})-\mathrm{CO}$ bond axis.

In the case of $\mathbf{P N C}\left[\mathbf{c C}_{60}-\mathbf{C o}-\boldsymbol{v} \mathbf{C O}\right]$ the $\mathrm{Co}^{-} \mathrm{C}_{\mathrm{CO}}$ distance was $0.18 \AA$ shorter (measuring 2.037(9) $\AA$ ), than that observed in $\mathrm{Co}_{2}$ (dobdc) which is again indicative of a weak $\mathrm{Co}-\mathrm{CO}$ interaction. For $\mathrm{PNC}\left[\boldsymbol{c C}_{\mathbf{6 0}}-\mathbf{C o}-\boldsymbol{v} \mathrm{CO}\right]$ the $\mathrm{Co}-\mathrm{C}-\mathrm{O}$ angle was constrained via symmetry to $180^{\circ}$, as the Co and CO molecule lie on a 4 -fold rotation axis. Nevertheless, the larger displacement parameters for the oxygen atom of the bound $\mathrm{CO}$ suggests a similar deflection along the $\mathrm{Co}-\mathrm{CO}$ bond axis. 
Upon increasing $\mathrm{CO}$ gas pressure to $6.4 \mathrm{bar}, \mathrm{PNC}\left[\mathrm{cC}_{60}-\mathbf{C o -} \boldsymbol{\nu} \mathbf{C O}\right]$ changed little structurally, however, the contents of the void increased steadily, Table 7.1. The CO binding was found to be fully reversible upon reapplying high vacuum at room temperature for 23 mins and was accompanied by a large reduction in the contents of the void. The ease of reversibility and reactivation of the open Co site $\mathbf{P N C}\left[\mathbf{c C}_{\mathbf{6 0}} \mathbf{C}^{-}\right.$ Co] could be promising for CO separations.

\subsection{2 $\mathrm{O}_{2}$ adsorption}

A second crystal of $\mathbf{P N C}\left[\mathbf{c C}_{60}-\mathbf{C o}\right]$ was loaded into the gas cell and immediately placed under high vacuum $\left(1 \times 10^{-6} \mathrm{mbar}\right)$ at room temperature for 20 minutes to evacuate the cell, lines, and void content. Data were then collected at $180 \mathrm{~K}$ while still under vacuum.

The dosing of $\mathrm{O}_{2}$ was incremental with changes in pressure made at room temperature while all data collections were conducted at $180 \mathrm{~K}$. The first dose of $\mathrm{O}_{2}$ was at a partial pressure of 0.1 bar. At this pressure there was a large increase in pore content from 2180 to $3443 \mathrm{e}^{-} /$unit cell (Table 7.2), however, despite the apparent uptake, $\mathrm{O}_{2}$ was not found bound to the open metal site. Increasing the pressure to 0.2 bar of $\mathrm{O}_{2}$ further increased the void content but again $\mathrm{O}_{2}$ was not observed binding to the metal.

On increasing to a partial pressure of 0.5 bar, $\mathrm{O}_{2}$ was observed binding to the metal site forming PNC $\left[\boldsymbol{c} \mathbf{C}_{60}-\mathbf{C o}-\boldsymbol{v} \mathbf{O}_{2}\right]$. The $\mathrm{Co}_{-} \mathrm{O}_{\mathrm{O}_{2}}$ distance measured 2.41(3) $\AA$, while the Co-O-O angle measured $112(3)^{\circ}$. The bent $\mathrm{O}_{2}$ binding geometry (i.e. deviation of the Co-O-O angle from $180^{\circ}$ ) leads to positional disorder due to the 4-fold axis. This geometry, referred to as an end-on configuration, has been predicted and observed experimentally through other techniques, but this is, as far as we are aware the first time it has been observed in a molecular porous material using in-situ X-ray diffraction. ${ }^{14}$ Unlike in the case of $\mathrm{CO}$, the site occupancy of the bound $\mathrm{O}_{2}$ was not $100 \%$. The occupancy of the $\mathrm{O} 2$ at 0.5 bar was refined to 0.462 . 
Table 7.2 - Summary of gas cell scXRD experiments using $\mathrm{O}_{2}$ gas.

\begin{tabular}{cccccc}
\hline $\begin{array}{c}\mathbf{O}_{2} \text { Pressure } \\
(\text { bar })\end{array}$ & Co-O $_{\mathbf{O}_{2}}(\mathbf{A})$ & O-O $(\mathbf{A})$ & $\begin{array}{c}\text { Co-O-O } \\
\text { Angle }\left({ }^{\circ}\right)\end{array}$ & O $_{2}$ Occupancy & $\begin{array}{c}\text { SQUEEZE } \\
(\mathbf{e}-/ \text { unit cell) }\end{array}$ \\
\hline $1 \times 10^{-9}$ & N/A & N/A & N/A & N/A & 2180 \\
0.1 & N/A & N/A & N/A & N/A & 3443 \\
0.2 & N/A & N/A & N/A & N/A & 4146 \\
0.5 & $2.41(3)$ & $1.204(10)$ & $112(3)$ & 0.462 & 3999 \\
1.0 & $2.110(17)$ & $1.195(10)$ & $151.3(11)$ & 0.505 & 4207 \\
8.1 & $2.066(16)$ & $1.205(10)$ & $145.5(13)$ & 0.707 & 4266 \\
$1 \times 10^{-9}$ & $2.30(6)$ & $1.212(10)$ & $119(6)$ & 0.448 & 1510 \\
\hline
\end{tabular}

On increasing the pressure of $\mathrm{O}_{2}$ in the cell the occupancy of the bonded $\mathrm{O}_{2}$ increased. This coincided with a reduction in the $\mathrm{Co}-\mathrm{O}_{\mathrm{O}_{2}}$ distance and increasing Co-O-O angle. By 8.1 bar, the highest pressure tested, the $\mathrm{Co}-\mathrm{O}_{\mathrm{O}_{2}}$ distance and Co-O-O angle measured 2.066(16) $\AA$ and $145.5(13)^{\circ}$ respectively with an occupancy of 0.707, Figure 7.3.

The Co- $\mathrm{O}_{\mathrm{O}_{2}}$ distances observed in $\mathbf{P N C}\left[\boldsymbol{c} \mathbf{C}_{\mathbf{6 0}}-\mathbf{C o -} \boldsymbol{v} \mathrm{O}_{2}\right]$ are longer than is typically found for cobalt dioxygen complexes. A search of the CSD shows that from 17 reported dioxygen adducts, most have a $\mathrm{Co}-\mathrm{O}_{\mathrm{O}_{2}}$ distance that falls below $2.0 \AA$. The only example with a Co- $\mathrm{O}_{\mathrm{O}_{2}}$ distance longer than $2 \AA$ was found in the MOF $\mathrm{Co}_{2}$ (dobdc) from Gonzalez et al. where they investigated the uptake of $\mathrm{O}_{2}$ in the same study as $\mathrm{CO}$ discussed above. For $\mathrm{Co}_{2}$ (dobdc) the $\mathrm{Co}-\mathrm{O}_{\mathrm{O}_{2}}$ distance and Co$\mathrm{O}-\mathrm{O}$ angle are reported as $2.216 \AA$ and $\sim 128^{\circ}$ respectively. Upon comparison to PNC $\left[\boldsymbol{c C}_{60}-\mathbf{C o}^{-v \mathrm{O}_{2}}\right]$ this is closer to the behaviour seen on exposure to low partial pressures of $\mathrm{O}_{2}$. Once the pressure of $\mathrm{O}_{2}$ was raised to 1 bar and above, the Co$\mathrm{O}_{\mathrm{O}_{2}}$ distance reduced significantly, and the Co-O-O angle increased from $\sim 120^{\circ}$ to $\sim 145^{\circ}$. This change could suggest a pressure mediated alteration to the bonding of the $\mathrm{O}_{2}$ to the $\mathrm{Co}$, however, this would require further investigation to probe the bonding interactions.

After the measurements at pressure were completed, high vacuum was reapplied to the sample at room temperature. After 5 mins of high vacuum data were collected at $180 \mathrm{~K}$. Interestingly, unlike $\mathrm{CO}, \mathrm{O}_{2}$ remained bound to the metal site 


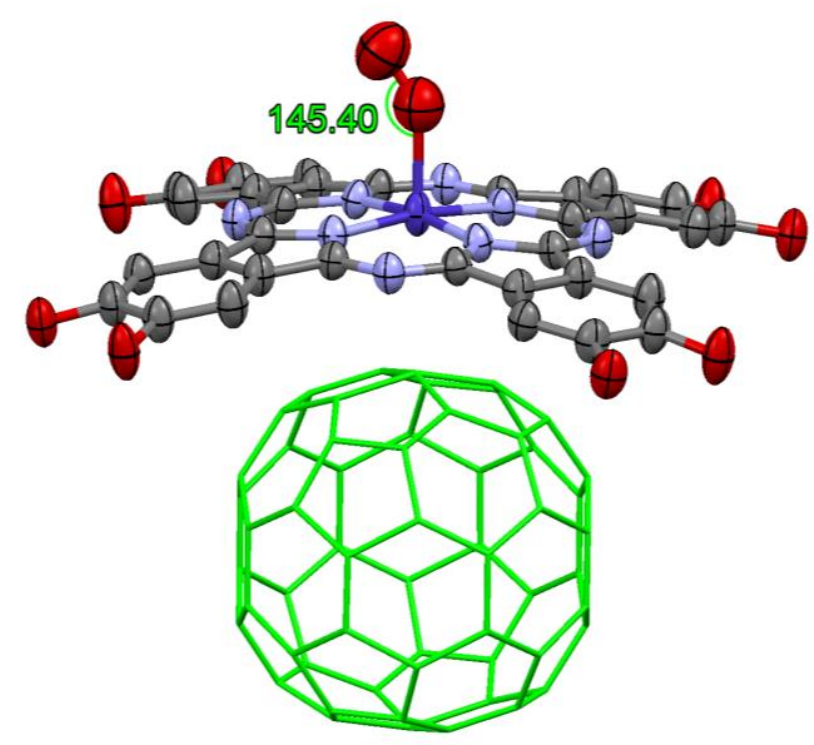

Figure 7.3 - Structure of $\mathbf{P N C}\left[\boldsymbol{c C}_{60}-\mathrm{Co}_{-}-\boldsymbol{v} \mathrm{O}_{2}\right]$ at a $\mathrm{O}_{2}$ gas pressure of $8.1 \mathrm{bar}$, clearly showing the bent, end-on binding geometry of the $\mathrm{O}_{2}$. The substituents groups on the phthalocyanine and hydrogens have been hidden for clarity. The C60 is shown green.

after evacuation, however the binding of the $\mathrm{O}_{2}$ reverted back to that observed at partial pressures of $\mathrm{O}_{2}$, with a much longer $\mathrm{Co}-\mathrm{O}_{\mathrm{O}_{2}}$ distance and more bent geometry. A very large reduction in void content was also observed and the occupancy of the bound $\mathrm{O}_{2}$ decreased (to 0.448).

Both the geometry, and binding strength are of interest as metal-phthalocyanines could potentially be used as possible oxygen reduction catalysts in fuel cells, however the exact functioning and deactivation of these catalysts is currently unknown. ${ }^{15}$ Though numerous techniques have been utilized to study $\mathrm{O}_{2}$ and other gas-binding mechanisms in phthalocyanines, such as X-ray photoelectron spectroscopy and X-ray absorption spectroscopy ${ }^{16}$, the $\mathbf{P N C}\left[\mathbf{c C}_{60}-\mathbf{C o}\right]$ sample studied here would appear to be idealised sample in order to study, crystallographically the interaction of gas molecules on an open metal site contained within a phthalocyanine. 


\subsection{Conclusions}

Here we have presented an in-situ diffraction study on the molecular porous material PNC $\left[\mathbf{c C}_{\mathbf{6 0}}-\mathbf{C o}\right]$ where single crystals of $\mathbf{P N C}\left[\boldsymbol{c C}_{\mathbf{6 0}}-\mathbf{C o}\right]$ have been exposed to both $\mathrm{CO}$ and $\mathrm{O}_{2}$ separately, whilst simultaneously collecting X-ray diffraction data. Removal of the axial water molecule bound to the Co centre under vacuum exposed an empty metal site in the void space of $\mathbf{P N C}\left[\mathbf{c C}_{60}-\mathbf{C o}\right]$. On increasing pressure, from 1 bar to 6.4 bar of CO, CO was found to readily bind with $100 \%$ occupancy to the open metal site with a linear geometry to form $\mathbf{P N C}\left[\mathbf{c C}_{60}-\mathrm{Co}_{-}\right.$ $\boldsymbol{v C O}$. Only the chemisorbed CO could be modelled crystallographically, though uptake of physisorbed CO was inferred by an increase in guest content modelled and quantified using the SQUEEZE algorithm. The Co-C $C_{\text {Co }}$ distance was over $2 \AA$, which is unusually long for Co carbonyl systems (which are typically $1.8 \AA$ ), and therefore indicative of a weak bond with little $\pi$ backbonding. On placing PNC $\left[\mathbf{c C}_{60}-\mathbf{C o -} v \mathbf{C O}\right]$ under vacuum, the bound $\mathrm{CO}$ could be easily removed. Uptake and reversible binding of $\mathrm{CO}$ at ambient temperature and low pressures could potentially lead to sensing applications, and we are currently investigating this.

$\mathrm{O}_{2}$ gas was also found to bind to the open metal site of $\mathbf{P N C}\left[\mathbf{c C}_{60}-\mathbf{C o}\right]$ at a partial pressures of 0.5 bar. The binding of $\mathrm{O}_{2}$ in $\mathbf{P N C}\left[\boldsymbol{c C}_{60}-\mathbf{C o -}-\boldsymbol{v} \mathrm{O}_{2}\right]$ was end on, binding through one of the $\mathrm{O}$ atoms, with a bent geometry. On increasing pressure, there appeared to be two binding modes; at partial pressures the Co-O-O angle was $120^{\circ}$ with a Co- $\mathrm{O}_{\mathrm{O}_{2}}$ distance of $\sim 2.35 \AA$, whereas at higher pressures the Co$\mathrm{O}_{\mathrm{O}_{2}}$ distance reduced to $\sim 2.08 \AA$ and the $\mathrm{Co}-\mathrm{O}-\mathrm{O}$ angle straightened to $\sim 148^{\circ}$. The occupancy of the $\mathrm{O}_{2}$ site was not fully occupied at any of these pressures, however the occupancy increased with pressure reaching a maximum of 0.71 at 6.4 bar. Unlike for $\mathrm{CO}$, the binding of $\mathrm{O}_{2}$ was found to be only partially reversible on evacuation. $\mathrm{O}_{2}$ binding in metal-phthalocyanines are of particular interest and this system has provided an opportunity to study these interactions crystallographically. 


\subsection{References}

1 Barbour, L. J. Crystal porosity and the burden of proof. Chemical Communications, 1163-1168, doi:10.1039/B515612M (2006).

2 Fairen-Jimenez, D., Moggach, S. A., Wharmby, M. T., Wright, P. A., Parsons, S. \& Düren, T. Opening the Gate: Framework Flexibility in ZIF-8 Explored by Experiments and Simulations. Journal of the American Chemical Society 133, 8900-8902, doi:10.1021/ja202154j (2011).

3 Carrington, E. J., Vitorica-Yrezabal, I. J. \& Brammer, L. Crystallographic studies of gas sorption in metal-organic frameworks. Acta Crystallographica Section B 70, 404-422, doi:10.1107/S2052520614009834 (2014).

4 Allen, F. The Cambridge Structural Database: a quarter of a million crystal structures and rising. Acta Crystallographica Section B 58, 380-388, doi:10.1107/S0108768102003890 (2002).

5 Moghadam, P. Z., Li, A., Wiggin, S. B., Tao, A., Maloney, A. G. P., Wood, P. A., Ward, S. C. \& Fairen-Jimenez, D. Development of a Cambridge Structural Database Subset: A Collection of Metal-Organic Frameworks for Past, Present, and Future. Chemistry of Materials 29, 2618-2625, doi:10.1021/acs.chemmater.7b00441 (2017).

6 Evans, J. D., Huang, D. M., Haranczyk, M., Thornton, A. W., Sumby, C. J. \& Doonan, C. J. Computational identification of organic porous molecular crystals. Crystengcomm 18, 4133-4141, doi:10.1039/C6CE00064A (2016).

7 Winter, G. xia2: an expert system for macromolecular crystallography da ta reduction. Journal of Applied Crystallography 43, 186-190, doi:10.1107/S0021889809045701 (2010).

8 Collaborative. The CCP4 suite: programs for protein crystallography. Acta Crystallographica Section D 50, 760-763, doi:10.1107/S0907444994003112 (1994).

9 Evans, P. Scaling and assessment of data quality. Acta Crystallographica Section D 62, 72-82, doi:10.1107/S0907444905036693 (2006).

10 Betteridge, P. W., Carruthers, J. R., Cooper, R. I., Prout, K. \& Watkin, D. J. CRYSTALS version 12: software for guided crystal structure analysis. 
Journal of Applied Crystallography 36, 1487-1487, doi:10.1107/s0021889803021800 (2003).

11 Spek, A. L. Single-crystal structure validation with the program PLATON. Journal of Applied Crystallography 36, 7-13, doi:10.1107/s0021889802022112 (2003).

12 Bruno, I. J., Cole, J. C., Edgington, P. R., Kessler, M., Macrae, C. F., McCabe, P., Pearson, J. \& Taylor, R. New software for searching the Cambridge Structural Database and visualizing crystal structures. Acta Crystallographica Section B 58, 389-397, doi:10.1107/S0108768102003324 (2002).

13 Gonzalez, M. I., Mason, J. A., Bloch, E. D., Teat, S. J., Gagnon, K. J., Morrison, G. Y., Queen, W. L. \& Long, J. R. Structural characterization of framework-gas interactions in the metal-organic framework Co2(dobdc) by in situ single-crystal X-ray diffraction. Chemical Science 8, 4387-4398, doi:10.1039/C7SC00449D (2017).

14 Holland, P. L. Metal-dioxygen and metal-dinitrogen complexes: where are the electrons? Dalton Transactions 39, 5415-5425, doi:10.1039/C001397H (2010).

15 Jasinski, R. Cobalt Phthalocyanine as a Fuel Cell Cathode. Journal of the Electrochemical Society 112, 526-528, doi:10.1149/1.2423590 (1965).

16 Miedema, P. S., van Schooneveld, M. M., Bogerd, R., Rocha, T. C. R., Hävecker, M., Knop-Gericke, A. \& de Groot, F. M. F. Oxygen Binding to Cobalt and Iron Phthalocyanines As Determined from in Situ X-ray Absorption Spectroscopy. The Journal of Physical Chemistry C 115, 2542225428, doi:10.1021/jp209295f (2011). 


\section{- Chapter 8 - \\ Concluding Remarks and Further Work}




\subsection{Concluding Remarks and Further Work}

A summary of the key results from this thesis will be presented below.

Chapter 3 was focused on the design, manufacture, testing, and optimisation of the sapphire capillary pressure cell for use on the small molecule beamline I19 at the Diamond Light Source. Here we presented many solutions to the practical aspects of integrating a new piece of apparatus on to a beamline. This included the custom mount to the goniometer, safety protocols for users, and methods to collect data with the accuracy and precision required to measure the small changes seen over this pressure range.

The compression of the small organic molecule HMTH and its deuterated version HMTD were investigated pressurising and depressuring from ambient to l000bar. From the very small changes in lattice parameters precise equations of state for both HMTH and HMTD were determined. Measurements of this type demonstrate the potential use of this cell in the development of new diffraction standards and pressure calibrants for high-pressure experiments.

This cell offers an enormous opportunity for future work as it reopens a regime of pressure long closed, during which time there have been great advances in the technology of single crystal X-ray diffraction. At the same time the variety, complexity, and compressibility of the materials/compounds scientists wish to study has greatly increased and some are not suitable for traditional high-pressure experiments using a DAC. This cell, in conjunction with the world-class instrumentation offered by Beamline I19, make it possible to investigate the initial response a compound has to pressure, in a pressure region of growing interest. There are many opportunities for future work with this cell as there is huge potential to investigate compounds that have been formerly disregarded for investigation at pressure due to their extreme compressibility. This is the case for many large and complex systems such as proteins and soft porous crystals.

The sapphire capillary pressure cell was used in Chapter 4 to investigate the structural behaviour of a small pore $\mathrm{MOF}\left(\mathrm{Sc}_{2} \mathrm{BDC}_{3}\right)$ below 1500 bar when exposed to $n$-pentane (np) and isopentane (ip). Solvent uptake into porous materials can 
induce large structural changes at 100's of bar. This work showed that the smaller size and shape of $n$-pentane versus isopentane facilitated the swelling and super filling of the pores of the framework. Swelling is a known important stabilisation phenomenon for MOFs at pressure and this is study presents one of the most detailed single-crystal X-ray diffraction investigations of this stabilisation effect.

$\mathrm{Sc}_{2} \mathrm{BDC}_{3}$ has been extensively investigated at high-pressure and this work shows how the sapphire capillary pressure cell can be used to complement and expand upon those results. This cell operates in a pressure range below that commonly attainable using DAC methods and should been considered as complementary to other high-pressure methods.

Chapter 5 presents the effect of pressure on a novel $\mathrm{Cu}$ based framework, CuPyr-I, using more conventional DAC high-pressure techniques. CuPyr-I was found to exhibit high-pressure and low-temperature phase transitions, a pressure induced Jahn-Teller switch (which was hydrostatic medium dependent), piezochromism, and negative linear compressibility. Although each of these phenomena has been reported numerous times in a range of materials, this is, to the best of our knowledge, the first example to have been observed within the same material.

One avenue of future work with this system would be to quantify the colour change of CuPyr-I on the applications of pressure through high-pressure UV/vis. In addition to this two isostructural analogues, replacing the $\mathrm{Cu}$ for $\mathrm{Hg}$ or $\mathrm{Mn}$, have been synthesised offering further investigation into these compounds.

The final two chapters, Chapters 6 and 7, present the exceptional thermal, chemical, and mechanical stability of a porous molecular crystal system (PMC) prepared by the co-crystallisation of a cobalt phthalocyanine derivative and a fullerene $\left(\mathrm{C}_{60}\right.$ or $\left.\mathrm{C}_{70}\right)$. The stabilising fullerene is captured in the cavity between two phthalocyanines in a ball and socket arrangement. These PMCs retain their porous structure: on the evacuation of solvent of crystallisation; on heating to over $500 \mathrm{~K}$; on prolonged immersion in boiling aqueous acid, base, and water; and at 
extreme pressures of up to $5.85 \mathrm{GPa}$. This study represents the first reported highpressure study of a PMC.

In-situ gas sorption studies demonstrated the stability of this system to high vacuum and the removal of an axial water to expose an open site on the Co. Once activated, the reactive Co cation is was found to be accessible via the massive interconnected voids as demonstrated buy the uptake, and chemisorption of CO and $\mathrm{O}_{2}$ to the empty metal site. In the case of $\mathrm{CO}$ this uptake was found to be readily reversible were as the bound $\mathrm{O}_{2}$ could only be partially removed.

In addition to providing exceptional stability, the co-crystallisation of cobalt phthalocyanine and a fullerene with this structure provides intimate contact between of one of the most studied photoelectron donors (phthalocyanine) with the most studied class of photoelectron acceptor (fullerene). The combination of these highly functional components within a porous material suggests an enormous number of future research opportunities and potential applications. For future experimental work these compounds can be made with an enormous number of different metal phthalocyanines and the porous nature of this structure make it potentially ideal for investigating the binding of different gasses to different metals.

In conclusion, this thesis has shown the development and use of a range of different crystallographic techniques to investigate porous materials at pressure. Each chapter takes a different technique or class of porous materials to show a further aspect of this field. The adaptation and development of new techniques runs throughout this thesis and as the materials that we want to investigate at pressure become ever more challenging, technique development will have to keep pace with these changes. 